\title{
With an eye on contact lenses : technological advancements in medical and optical applications
}

Citation for published version (APA):

de Brabander, J. (2002). With an eye on contact lenses : technological advancements in medical and optical applications. [Doctoral Thesis, Maastricht University]. Universiteit Maastricht. https://doi.org/10.26481/dis.20020530jb

Document status and date:

Published: 01/01/2002

DOI:

10.26481/dis.20020530jb

Document Version:

Publisher's PDF, also known as Version of record

\section{Please check the document version of this publication:}

- A submitted manuscript is the version of the article upon submission and before peer-review. There can be important differences between the submitted version and the official published version of record.

People interested in the research are advised to contact the author for the final version of the publication, or visit the DOI to the publisher's website.

- The final author version and the galley proof are versions of the publication after peer review.

- The final published version features the final layout of the paper including the volume, issue and page numbers.

Link to publication

\footnotetext{
General rights rights.

- You may freely distribute the URL identifying the publication in the public portal. please follow below link for the End User Agreement:

www.umlib.nl/taverne-license

Take down policy

If you believe that this document breaches copyright please contact us at:

repository@maastrichtuniversity.nl

providing details and we will investigate your claim.
}

Copyright and moral rights for the publications made accessible in the public portal are retained by the authors and/or other copyright owners and it is a condition of accessing publications that users recognise and abide by the legal requirements associated with these

- Users may download and print one copy of any publication from the public portal for the purpose of private study or research.

- You may not further distribute the material or use it for any profit-making activity or commercial gain

If the publication is distributed under the terms of Article $25 \mathrm{fa}$ of the Dutch Copyright Act, indicated by the "Taverne" license above, 


\section{With an Eye on Contact Lenses}

Technological Advancements in Medical and Optical Applications

John de Brabander 
With an Eye on Contact Lenses

Technological Advancements in

Medical and Optical Applications

\section{Proefschrift}

ter verkrijging van de graad van doctor aan de Universiteit Maastricht, op gezag van de Rector Magnificus Prof. dr A.C. Nieuwenhuijzen Kruseman volgens het besluit van het College van Decanen, in het openbaar te verdedigen

op donderdag 30 mei 2002 om 14.00 uur

door

John de Brabander

geboren op 22 september 1946 te Pijnacker. 
Promotor:

Prof. dr. F. Hendrikse

Co-Promotores:

Dr. R.M.M.A. Nuijts

Dr. F.H.M. Jongsma

Beoordelings-commissie:

Prof. dr. ir. L.H. Koole (voorzitter)

Dr. G.L. van der Heijde (Vrije Universiteit, Amsterdam)

Prof. dr. H.C. Hemker

Prof. dr. A. Kijlstra (Universiteit van Amsterdam)

Prof. dr. H. Kingma

John de Brabander

With an Eye on Contact Lenses

Technological Advancements in Medical and Optical Applications

(C) Copyright 2002 ERIM Publications

All rights reserved. No part of this book may be translated or reproduced in any form by print, photo print, microfilm, or any other means without prior written permission of the publisher.

ISBN 9056811371

De uitvoering en publicatie van dit werk is mogelijk gemaakt door het Eye Research Institute Maastricht (ERIM).

Vormgeving en printwerk: Unigraphic, Universiteit Maastricht. 
"Es ist hier die Rede nicht von einer durchzusetzenden Meinung, sondern von einer mitzuteilenden Methode, deren sich ein jeder als einer Werkzeuges nach seiner Art bedienen möge."

J.W. von Goethe in a letter to G.W.F. Hegel,

explaining his scientific approach in studying the phenomena of colour.

To all interested in vision care

In memoriam to Jan H.C. Kok 


\section{Contents}

$\begin{array}{lll}9 & \text { Chapter } 1 & \text { General Introduction } \\ 10 & 1.1 & \text { Preface } \\ 11 & 1.2 \text { Introduction to the studies } \\ 31 & 1.3 \text { Aims of the studies } \\ 31 & 1.4 \text { Outline of this thesis }\end{array}$

Chapter 2 Contact Lenses in Infant Aphakia and as Artificial Iris

2.1 Introduction

2.2 Silicone contact lenses in infant aphakia A practical approach to and long-term results of fitting silicone contact lenses in aphakic children after congenital cataract.

2.3 Coloured contact lenses as artificial iris The use of standard thin high water content artificial iris and black pupil contact lenses.

Chapter 3 High Gas Permeable Rigid Contact Lenses

3.1 Introduction

3.2 HGP contact lenses, material and design Low or high Dk? Spherical or aspherical rigid gas permeable lenses? The gap between theory and practice.

3.3 HGP contact lenses, fitting and care systems Evaluation of a new system of lens parameter selection and comparison of traditional vs one-step lens care systems for aspheric high-Dk RGP contact lenses.

4.2 Soft toric contact lenses Clinical evaluation of a custom-made toric soft lens system. Presbyopia and contact lenses: a literature review and a clinical study on discriminant factors in varifocal soft contact lens fitting. 
4.4 Aspheric contact lenses in high ametropia Contrast sensitivity with soft contact lenses compensated for spherical aberration in high ametropia. Simulated optical performance of custom wave-front soft contact lenses for keratoconus.

155 Chapter 5 Measuring Techniques

\section{Chapter 6 Summary and Conclusions}

Publications

5.3 Pupillometry

5.4 Tear film fluorescence

5.2 Topography of the anterior eye surface

5.2.1 Development of a wide field height eye topographer: validation on models of the anterior eye surface.

5.2.2 Height measurements of the corneal-scleral profile using the Maastricht shape topographer. Infrared pupillometry in presbyopes fitted with soft contact lenses.

Testing fluorescein agents as a diagnostic tool and a coating for anterior eye topography: a feasibility study.

\section{List of abbreviations and terms}


Chapter 1

General Introduction 


\subsection{Preface}

\section{Contact lenses and technological developments}

The field of contact lenses (CLs) is linked with technical science by incorporating new developments in technology ${ }^{1}$ and by investigating the clinical application of products that result. In CL science, the "traditional view" is represented by the techniques and methods that are used in daily practice. With the rapid developments in technology, many new ideas arise, but also old ideas that could not be successfully put into practise before might, by using modern techniques, become possible. Especially for the latter group, extensive scientific action is needed to prove its validity against the inherently stubborn traditional view. ${ }^{2}$

\section{Visual correction and contact lenses}

The most common reason for compromised visual acuity in humans is of optical nature. If the optical power of the eye is not in line with its axial length, images will not be focussed on the retina. These refractive errors (ametropia) can be corrected by placing a spectacle lens at a certain distance in front of the eye. Also, the ability of the eye to focus at shorter distances (accommodation) that diminishes with age (presbyopia) can be compensated by the use of reading glasses. If the refractive error is not too high people see well with spectacles but most feel constrained in wearing them.

CLs fulfil the same purpose in correcting refractive error as spectacle lenses but are placed in the tear film very close to the front surface of the eye (cornea). In fact the front surface of the eye is replaced by the front surface of the CL which has many optical advantages. The most important reasons for ametropes to prefer wearing CLs are that they are no longer hindered by the spectacle frame and that the correction of their ametropia is invisible. Despite this, only $3 \%$ of spectacle wearers use CLs on a regular basis. Apart from economical factors, one of the reasons for this relatively low figure is that CLs need to be simpler and more convenient to use. ${ }^{3}$ Within the last three decades the contact lens industry has focussed on providing safe, effective, comfortable and easy to use CLs to correct simple ametropia. The ultimate result has been the availability of daily disposable CLs.

In essence, ametropes have one desire: "wake up and see". To fulfil this desire attempts are made to permanently correct ametropia by CLs (extended wear), by changing the front surface of the eye using CLs (ortho-k) or by invasively removing corneal tissue (refractive surgery). Despite recent improvements with these techniques, the clinical results could not convince the profession to drastically change their "traditional view" of using spectacle lenses or daily wear contact lenses. Also, patient surveys indicate that the majority of CL wearers would prefer continuous wear CLs over invasive techniques, if the lenses were safe, effective and comfortable. ${ }^{3}$

At present, research in the CL field is directed towards developing safe continuous wear, to use advanced technology to correct effectively more complicated cases of refractive error, and to provide prosthetic aids for the eye. The latter two areas of research are the topic in this thesis. 


\subsection{Introduction to the studies}

\section{Contact lens history at a glance}

Although the idea of substituting the cornea by another refractive surface reaches back to Leonardo da Vinci ${ }^{4,5}$ in 1508 (Fig. 1a) and Descartes ${ }^{6,7}$ in 1637 (Fig. 1b), the clinical application of CLs started no earlier than around one hundred and twenty years ago. In 1887 Saemisch and Müller ${ }^{8}$ developed a wearable glass shell that was used as a protective device for the cornea. The term CL (Kontaktbrille) was first used by Fick in $1888^{9}$ in relation to optical correction of irregular corneas like in keratoconus. These scleral contact lenses consisted of large hemispheres made of blown glass ${ }^{10-13}$ and wearing time was usually around two hours after which anoxia and probably also adherence of the lens to the eye (lens binding) resulted in severe corneal oedema. The introduction of polymethyl methacrylate (PMMA) in $1935^{14}$ and corneal contact lenses made thereof around $1950^{15}$ significantly increased the use of CLs. Early work with these "hard" CLs was predominantly focussed on improving the geometry in order to keep these relatively small lenses on the eye (Fig. 1c) and limit physiological interaction with the tissues of the anterior eye. ${ }^{16-23}$
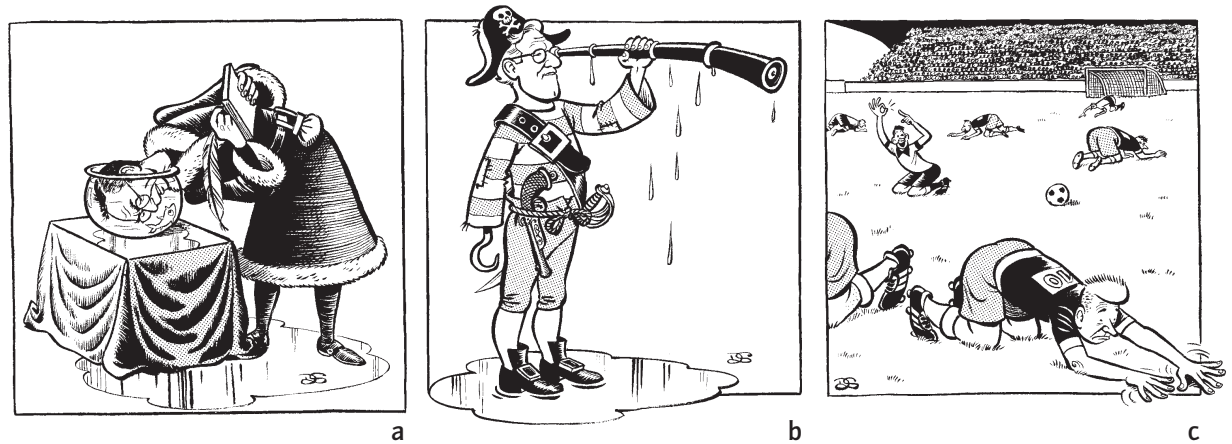

Figure 1. Artist's impression of the original ideas of Da Vinci (a) and Descartes (b) to replace the corneal surface by one with other optical power and the problem of keeping the relative small early corneal contact lenses on the eye (c). Original drawings by Dirk Stallaert as an exclusive contribution to this thesis made possible by Frans and Magda Jongsma.

With the introduction of poly(2-hydroxyethyl methacrylate) (PHEMA) around 196024 a new era in the use of CLs started. The original soft hydrophilic CLs made of a cross linked PHEMA contained $38.6 \%$ water in the hydrated state. These lenses were relatively thick, difficult to reproduce and the material showed chemical instabilities resulting in tearing and discoloration. ${ }^{25}$ These "soft" contact lenses (SCLs) were either produced with a revolutionary technique called spin casting 26 in which liquid monomers were injected into a rotating mould or by the method traditionally in use for PMMA in which a polymerised rod of material is cut into slices (buttons) to be further lathe cut and polished. In both methods a dehydrated CL is the semi-finished product which, after a process of washing out waste 
monomers, hydration, and sterilisation, becomes a wearable SCL. The milestones in the development of CLs are summarised in Table 1.

Table 1. Seven phases in the development of contact lenses

\begin{tabular}{|c|c|}
\hline $\begin{array}{l}\text { Phase } 1 \text { until } 1880 \\
\text { Theoretical idea }\end{array}$ & $\begin{array}{lr}\text { Theoretical pioneering } \\
\text { Leonardo da Vinci } & 1508 \\
\text { Rene Descartes } & 1637 \\
\text { Philip de LaHire } & 1685 \\
\text { John Hershel } & 1845 \\
\end{array}$ \\
\hline $\begin{array}{l}\text { Phase } 2 \text { 1880-1930 } \\
\text { First experiments }\end{array}$ & $\begin{array}{lr}\text { Clinical pioneering } & \\
\text { Fick } & 1888 \\
\text { Mueller } & 1889 \\
\text { Kalt } & 1889 \\
\end{array}$ \\
\hline $\begin{array}{l}\text { Phase } 3 \text { 1930-1950 } \\
\text { Clinical applications: } \\
\text { Scleral lenses } \\
\text { Special cases } \\
\text { PMMA as a material }\end{array}$ & $\begin{array}{l}\text { Restoring visual acuity } \\
\text { Clinical application mainly: } \\
\text { in irregular corneas and high ametropia } \\
\text { Topography with eye impressions } \\
\text { Improved haptic design } \\
\text { Material from glass to PMMA } \\
\end{array}$ \\
\hline $\begin{array}{l}\text { Phase } 4 \text { 1950-1975 } \\
\text { Corneal lenses }\end{array}$ & $\begin{array}{l}\text { Fitting normal ametropia } \\
\text { Material PMMA } \\
\text { From mono to multi-curve geometry } \\
\text { Advancement in lathe manufacturing } \\
\text { Increase in wearing time }\end{array}$ \\
\hline $\begin{array}{l}\text { Phase } 5 \text { 1962-recent } \\
\text { Soft hydrophilic lenses }\end{array}$ & $\begin{array}{l}\text { Fitting for comfort } \\
\text { Material HEMA } \\
\text { Manufacturing, lathe, spin-cast and moulding } \\
\text { Development of safety standards } \\
\text { Development of special care systems } \\
\text { Problems with service life }\end{array}$ \\
\hline $\begin{array}{l}\text { Phase } 6 \text { 1975-recent } \\
\text { Rigid gas permeable lenses }\end{array}$ & $\begin{array}{l}\text { Fitting for physiology } \\
\text { Research in corneal physiology } \\
\text { Development of oxygen permeable materials } \\
\text { Use of aspheric lens designs } \\
\text { Possibilities of extended wear }\end{array}$ \\
\hline $\begin{array}{l}\text { Phase } 7 \text { 1985-recent } \\
\text { Choice from a variety of rigid and soft } \\
\text { contact lens materials and designs }\end{array}$ & $\begin{array}{l}\text { Fitting with technology and marketing } \\
\text { From keratometer to eye topography } \\
\text { CNC sub-micron lathe manufacturing } \\
\text { Mass production with moulding } \\
\text { Use of frequent replacement systems } \\
\text { Optimising optics and fitting presbyopia }\end{array}$ \\
\hline
\end{tabular}

A remarkable event in CL history was the fact that in 1968 the US Food and Drug Administration (FDA) declared SCLs to be a drug. In March 1971 Bausch \& Lomb (Rochester, NY) gained FDA approval to market a relatively small, thin SCL (Soflens, Polymacon) produced with the refined method of spin casting and a heat method for disin- 
fecting. Meanwhile manufacturers in Europe (mainly UK and Germany) developed materials and techniques to produce SCLs based on traditional lathe cutting. For maintenance and disinfecting special chemical solutions were developed (cold disinfecting). The increased comfort and wearing time have made SCLs an extremely popular mode to correct normal ametropia as an alternative to spectacles (so-called cosmetic wear). Due to the hydrophilic nature of the material, the complete coverage of the cornea and the relative minor tear exchange under the lens, a variety of specific adverse effects have been reported. Among these are evenly distributed stromal oedema ${ }^{27}$, refractive error change ${ }^{28}$, changes in corneal thickness ${ }^{29}$, problematic oxygenation of the cornea in relation to hydration and lens thickness ${ }^{30}$, desiccation staining 31 , chemical keratopathy ${ }^{32}$ and a variety of delayed responses due to borderline conditions of hypoxia. ${ }^{33}$ One should recognise here that CL researchers have contributed substantially to fundamental knowledge on corneal physiology by studying the effects of CLs. ${ }^{22,34-45}$ During the 1970s and 1980s a variety of higher water content SCL materials were developed mainly based on the addition of N-vinyl pyrrolidone (NVP). 46 Relatively thin lenses produced from these materials have been shown to be advantageous in corneal response with respect to oxygenation. ${ }^{47}$ However, the idea that oxygen permeability would allow for overnight (permanent or extended) wear is, on theoretical grounds, not advisable with presently available SCL materials $s^{48}$ and carries a relatively high risk to develop a microbial keratitis. 49,50 Despite these facts extended wear hydrophilic CLs became quite popular in some countries. Inherent to hydrophilic materials is the denaturation of proteins on the lens surface, which can result in an immunological reaction of the palpebral conjunctiva called giant papillary conjunctivitis (GPC). In early days enzyme tablets, surfactant cleaners and oxidative products were used in attempts to keep the service life of the lens up to a reasonable level. With decreasing costs due to mass production today, there is a positive trend to replace all but especially hydrophilic CLs more frequently, the ultimate being a daily disposable soft lens first introduced in 1998 by Vistacon (one day Acuvue, Johnson \& Johnson, Jacksonville). During the past few years a variety of special SCLs have been developed. Among these are toric CLs to correct astigmatism ${ }^{51}$, multifocal CLs to correct presbyopia ${ }^{52}$, coloured CLs as iris prosthesis or to aid in photophobia ${ }^{53}$, so-called "fun lenses" to change eye colour, and thin plano lenses (bandage) ${ }^{54}$ or large scleral CLs $s^{55}$ to be used in cases of dry eyes, pathology or trauma. ${ }^{56}$

Silicone rubber (poly dimethyl siloxane) has been used since 197057 as a CL material. The advantage lies in its extremely high oxygen permeability making it suitable for extended wear. ${ }^{58}$ Because of the hydrophobic nature of the material, a surface treatment to optimise wetting is necessary. The main problems with silicone CLs are a relative short service life and the formation of deposits.59 Recently, hydrophilic materials with higher oxygen permeability have been developed by mixing silicone compounds into the polymer. ${ }^{60,61}$ Although the application of these new lenses remains to be investigated on a large scale, the first results point to metabolic success and safety in relation to 30 days continuous wear. ${ }^{3}$

Rigid Gas Permeable (RGP) materials were introduced around 1975.62,63 The first materials that were used showed only minor oxygen permeability and problems with deformation and deposit adherence. ${ }^{64-66}$ From 1975 till today many new RGP materials were introduced in which specific monomers are added to the polymer in order to enhance oxygen perme- 
ability, surface wetting and deposit resistance. ${ }^{46,67}$ Studies using high gas permeable (HGP) rigid CLs have shown favourable results in extended wear compared to hydrophilic lenses ${ }^{68,69}$ due to the ability to meet oxygenation levels of the cornea under closed eye conditions ${ }^{42}$, a more efficient tear exchange and fewer complications in relation to stubborn deposits. ${ }^{70}$ Reported complications with RGP CLs, especially under extended wear conditions, are lens adherence to the cornea (lens binding) ${ }^{71}$ and 3 and 9 o'clock desiccation staining. ${ }^{72}$ The availability of HGP materials has led to the successful revival of scleral lenses ${ }^{73,74}$ and to a system known as "piggy back" in which a soft CL is combined with a rigid CL to correct severe cases of corneal irregularity as in keratoconus. ${ }^{75}$

Next to the development of CL materials, new methods and instruments were also developed to improve measuring the shape of the eye. ${ }^{76-82}$ More precise information on the shape of the eye has renewed attention to the design of CLs resulting in the use of aspheric CLs ${ }^{83,84}$ with enhanced edge profiles. ${ }^{85}$

Recently, the development of submicron lathe machines enables the production of CLs of almost any shape and of any material. Using this new technology attempts are made to use topographic data of the individual eye to custom-design posterior CL surfaces. In addition, it has become possible to design anterior CL surfaces to correct for astigmatism, corneal irregularities like in keratoconus, presbyopia or higher order aberration of the eye.

An interesting fact in the development of CLs seems to be that applications, as described above, have been suggested long before technical production was possible. In a textbook by Filderman and White from 196986 the idea of using aspheric CLs including correction for spherical aberration of the eye is given in chapter 11 under the interesting title "new developments". The same was true for the principle description of scleral lenses by Herschel in 1827 , although it took some 60 years before the first CL was actually produced. ${ }^{87}$

\section{Contact lens usage}

Within the ophthalmic profession CLs are a normal integrated part of the practice and have evolved from "miracle optical things" to economically interesting products. In recent years more figures have become available about the market of CLs worldwide. Traditionally, figures about CL usage are based on production figures as supplied yearly by surveys among members of manufacturers associations like CLMA, NAC and EFCLIN. Since 1980 market investigation institutions like Intomart, EIM/HBD, and IfD/GfK have yearly been gathering retrospective data on contact lens usage through consumer surveys. More recently, surveys have been conducted that include analysis of the prescribing habits of practitioners ${ }^{88}$ and the economic viability of the investigated population. ${ }^{89} \mathrm{~A}$ problem in evaluating the available figures is that methods to gather the data vary among countries and time periods. Moreover, for some items estimations rather than measurements are used. Nevertheless, from the available studies a good impression can be deduced about the CL market today. A description of the various sources that we used to gather market figures can be found in the list of abbreviations.

The total number of CL wearers in the world is estimated to be around 80 million, growing by $4-5 \%$ per year (source IACLE, 1999). CLs represent at average only $3 \%$ of the estimated 2.5 billion spectacle wearers. The major explanation for this relatively low figure is 
economic viability. For $80 \%$ of the population on earth CLs can be categorised as unaffordable luxury products..$^{90}$ The other part of the explanation is that despite good marketing prospectives ${ }^{91,92}$ the use of CLs by presbyopes (around $50 \%$ of the population that need an optical correction) has during the last decade only grown from around 1 to $5 \%$ of all CLs ordered (source, Euromcontact, 1999).

More than $65 \%$ of CL wearers are female. ${ }^{88,89,93}$ The most important reason to wear CLs is to correct ametropia as a cosmetic alternative to spectacles $(80 \%)$; other reasons are high ametropia and astigmatism (6\%), changing eye colour (5\%), corneal irregularities (3\%), myopia control (3\%), post-refractive surgery (2\%) and therapeutic applications (1\%).

CL usage varies greatly per country. Recently Loan ${ }^{89}$ used an interesting way to put figures on CL usage per country in perspective by integrating percentages of people that are economically viable to CL wear. The penetration rate (Fig. 2) of CLs related to the adult population is above $11 \%$ in the US, the Netherlands and Japan, but much lower for other countries (e.g. UK 4.9\%, Germany 5.1\%, France 3.2\%, Korea 2.5\%, China 2.3\%, Brazil 1.1\%). If corrected for economical viability, these figures become very interesting. In the US, the Netherlands and Japan the figures are still the highest $(23,20.1$ and $18 \%$, respectively). Extremely different is the situation in China where only $2.3 \%$ of the adult population is wearing CLs but this is $15 \%$ of the economically viable population. To a lesser degree the UK shows this pattern too ( $4.9 \%$ of adult population compared to $11 \%$ of economically viable population). In Europe, Germany and France show relatively low figures on CL wear within the economically viable population with 9 and $6 \%$, respectively. This is comparable with Korea and Brazil with 8 and 9\%, respectively.

The type of CLs used also varies per country (Figs. 3 a,b,c). In all markets the traditional PMMA hard lens has been replaced. 89,93 In 1999 in the Netherlands and Germany relatively high percentages ( $42 \%$ and $20 \%$, respectively) of RGP CLs are used compared to USA and UK ( $10 \%$ and $14 \%$, respectively). $88,89,93,95$ What is remarkable is that, although the percentage of RGP CLs fitted in these countries is very different, the use shows a very similar pattern of a slight decline during the last five years. Morgan and Efron ${ }^{94-1}$ reported that following the legal restrictions in the use of trial lenses in the UK only 1 in 16 new contact lens fits were RGPs. However, factory gate figures on rigid lens sales in the UK do not show such a dramatic decline. Edwards and Hough ${ }^{44-2}$ conclude that RGP lenses will continue to secure a significant and profitable share of the contact lens market. This debate typically represents the difficulty in interpretation of market figures using various sources and measurements.

In all markets there is a significant shift towards the use of disposable (daily or weekly) and frequent replacement (one to 6 months) SCLs compared to traditional SCLs.

The tendency to use mass production of low cost, disposable CLs has positively shown that many people have started to wear CLs. However, the drop-out rate of wearers in high penetration markets is estimated to be around 15\% (source CLMA, 1999). There is also a tendency to use CLs occasionally and combined with spectacles (multiple ownership, Euromonitor, 1999). Reasons for drop-out are in order of importance, side effects, convenience, and comfort. Costs are ranked as being of minor importance. 89,93 


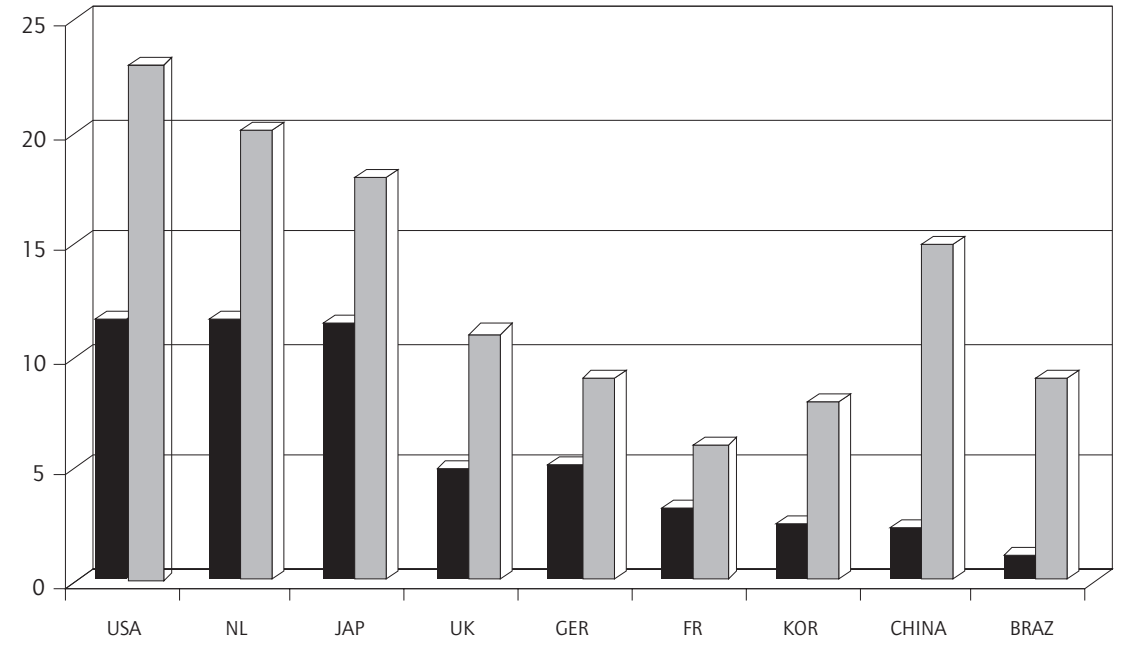

$\%$ CL Population $\quad \square \%$ CL Economic Viable Population

Figure 2. Usage of contact lenses in various countries as $\%$ of adult population and as $\%$ of economically viable. After Loan ${ }^{89}$, NL computed by us.

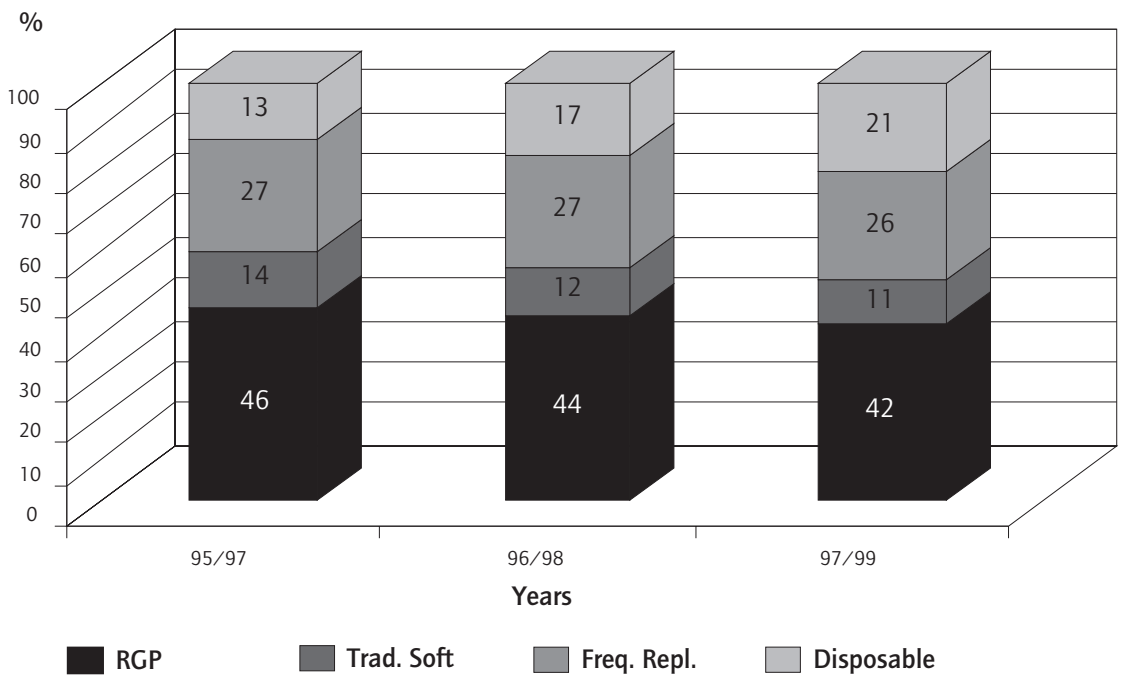

Figure 3a. Type of contact lenses used in the Netherlands from 1995 to 1999. 


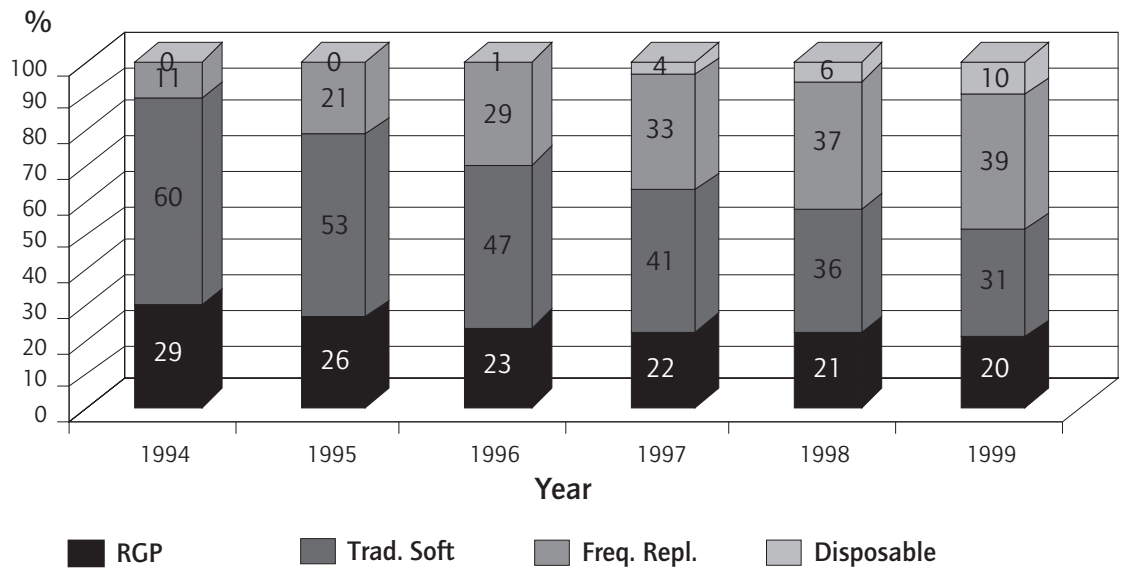

Figure 3b. Type of contact lenses used in Germany from 1994 to 1999.

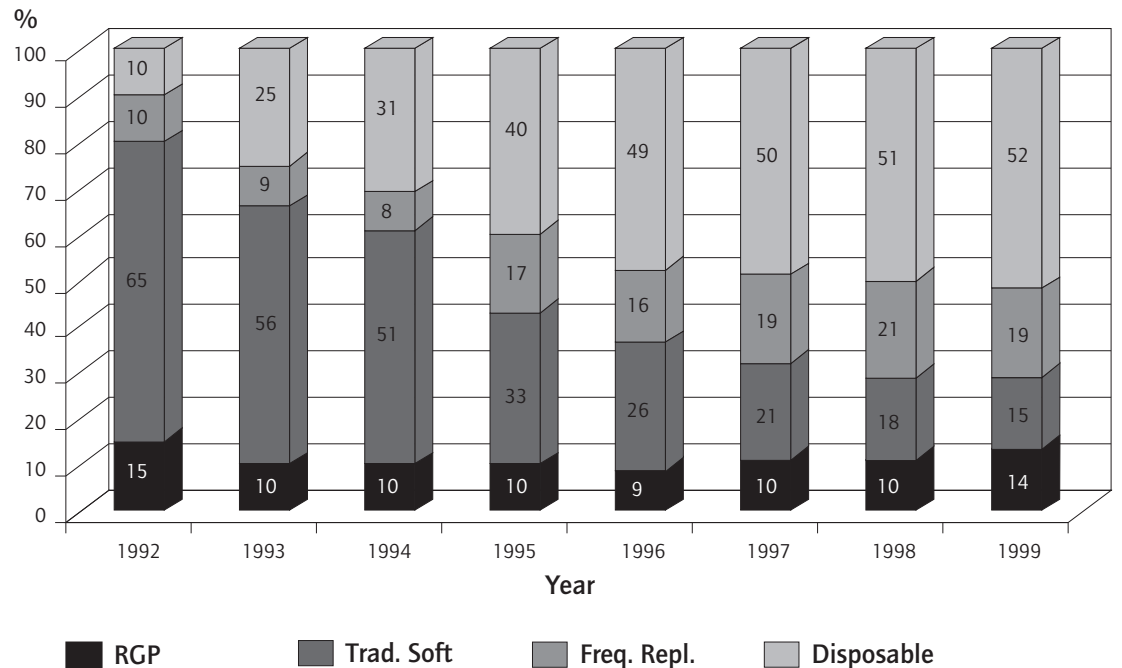

Figure 3c. Type of contact lenses used for new fits in the UK from 1992 to 1999.

In conclusion, it seems clear that the use of CLs is related to economic viability and cosmetic consciousness. Reasons to continue wearing are comfort and ease of use. The type of CLs used is strongly dependent on the advising and prescribing habit of the practitioner. The differences in CL wear between economically viable countries is probably related to the variability in regulations for prescribing CLs, differences in practitioners' education ${ }^{96}$ and a different approach of the ophthalmic profession towards CLs. ${ }^{89,93}$ Costs of CL wear seem to play a minor role in economically viable countries. ${ }^{89,93}$ 


\section{Contact lens wearing schedules}

With the development of biocompatible materials and better geometry, the wearing time of CLs has increased from a few hours (limited wear) as with the first glass scleral lenses to all waking hours (daily wear) with most rigid and soft CLs used today. With the introduction of higher water content hydrophilic and rigid gas permeable materials, experiments were started to wear contact lenses overnight for long periods. This so-called permanent wear of CLs in the normal population has not been very successful. It has been shown that oxygenation of the cornea under closed eye conditions (sleep) demands a CL with very high permeability to oxygen in order to keep up normal metabolism.42,97-101 Furthermore, the formation of deposits on the lens and debris between the CL and the corneal epithelium is an obstacle for permanent long term wear. Both problems together can lead to a permanent situation of hypoxia with the danger of weakening the normal defensive mechanisms of the eye. This compromised corneal condition can lead to serious complications as microbial keratitis. ${ }^{49}$ Incidence figures $50,102,103$ show that the risk of developing a microbial keratitis with SCLs under permanent wear conditions $(20 / 10,000)$ is 6 times higher compared to daily wear $(3.5 / 10,000)$. Microbial keratitis is also reported with RGP CL wear ${ }^{68,69}$ but the incidence $(1 / 10,000)$ is 20 times lower than with permanent wear hydrophilic CLs. ${ }^{50}$ Recently Cheng 50 showed that the incidence of microbial keratitis despite shortening the period of permanent wear has not decreased. Despite encouraging results with recently developed silicone hydrogels $s^{57}$, the general opinion of practitioners and researchers is that even with special HGP materials the wearing of CLs overnight should be limited to a week at the most ${ }^{70}$ and rest periods (e.g. 24 hours) are favourable to avoid problems in the long run. Moreover, it is usually recommended to extend normal daily wear only if absolutely necessary. With this, the term extended wear (EW) has been introduced as a safe option in selected cases combined with regular follow-up by the practitioner. ${ }^{104}$ Exceptions are the medical indicated permanent wear of CLs as therapeutic bandage 105 and silicon CLs in young children. The latter application is one of the topics in this thesis (chapter 2.2).

\section{Contact lens maintenance and replacement}

From CL research it is known that wetting prior to placement on the eye increases comfort, that during wear adherence of tear substances takes place and that the CLs could become contaminated especially if not worn for longer periods. ${ }^{106-111}$ Patient compliance to CL care has been indicated as an important factor to safe CL wear. ${ }^{112-115}$ This has led to the constant development of special CL care products for wetting, cleaning and disinfecting/storage. ${ }^{116}$ Since all three actions are needed, manufacturers offer the products as so-called CL care systems. With the introduction of new materials having entirely different properties, toxicity or hypersensitivity to substances in disinfecting, wetting or cleaning solutions have been reported. ${ }^{117-123}$ For this reason the FDA initially restricted the disinfecting method for SCLs to heat, using specially designed thermal units. A drawback of this method has been the heavy formation of deposits of different nature ${ }^{124,125}$ that decreases the service life of the SCL. In addition, the build up of denatured proteins can lead to immunological responses like giant papillary conjunctivitis. ${ }^{126,127}$ From 1975 to 1985 many alternative cleaning methods for SCLs have been introduced. Among these were enzymatic cleaning tablets, sur- 
factant and oxidative cleaners and many chemical (cold) disinfection solutions. Usually the practitioner selects the most suitable care system and provides a starter kit of the system with instructions to the patient. Low compliance of the patients is the main hazard to safe CL wear in the long run. ${ }^{113,128-131}$ To increase patients' compliance in the maintenance of CLs, care products are developed that combine cleaning, wetting and disinfecting actions. The safety and effectiveness of one of these so-called all-in-one or one-step solutions for RGP CLs is subject of this thesis (chapter 3.3).

The development in CL materials has moved in the direction of increased biocompatibility and higher oxygenation of the eye. Modern CL materials in soft and rigid modalities have many positive properties as compared to PMMA, but since these materials have become more complicated their service life is shorter. With decreasing manufacturing costs frequent replacement schemes have become available. In relation to the suitability for the patient, CLs are replaced between six months or one day (daily disposable). The latter option has been one of the major positive breakthroughs in modern CL usage. However, not all patients are suitable for this option for economic or ophthalmic reasons. In modern CL practice an important task of the practitioner is to advise the individual patient on choosing the optimal type of CL and the best suitable scheme of wearing and replacement. Throughout this thesis attempts are made to aid the practitioner in this process by reporting on modern fitting procedures, use of new CL designs and objective schemes to evaluate the performance of the $\mathrm{CL}$ on the eye.

\section{Contact lens materials, properties, classification and terminology}

Classification and terminology of CLs have developed over the years. Each step in the introduction of new materials, the geometry and fitting methods revealed new terms. Consequently, the indications, wearing schedules, maintenance / replacement schemes developed too and for these items many new terms were also introduced. Some historical terms are still in use although they do not completely cover modern applications or terms in technology. Sometimes this is confusing. To overcome this confusion CLs have started to become subject to international normative descriptions and can be classified from different angles:

\section{Material properties and manufacturing}

The use of plastics as CL material have made it possible to manipulate the properties of the material. The major properties for a CL material are refractive index, optical transmittance, hardness, scratch resistance, tensile strength, toxicity, gas permeability, water content, surface wettability, cleaning properties, flexibility and machinability. Optimising all these properties is a difficult task in developing CL materials since some are inherently counterproductive.

PMMA (Fig. 4a) was the first material introduced as an alternative to glass. Today this material is referred to as the classical "hard lens". This term, however, was invented only after the introduction of a modification to PMMA, known as the "soft contact lens". Before the introduction of the latter material (showing high flexibility and softness) CLs were simply called "contact lenses". Confusing terms were introduced in the 1970s with very thin 

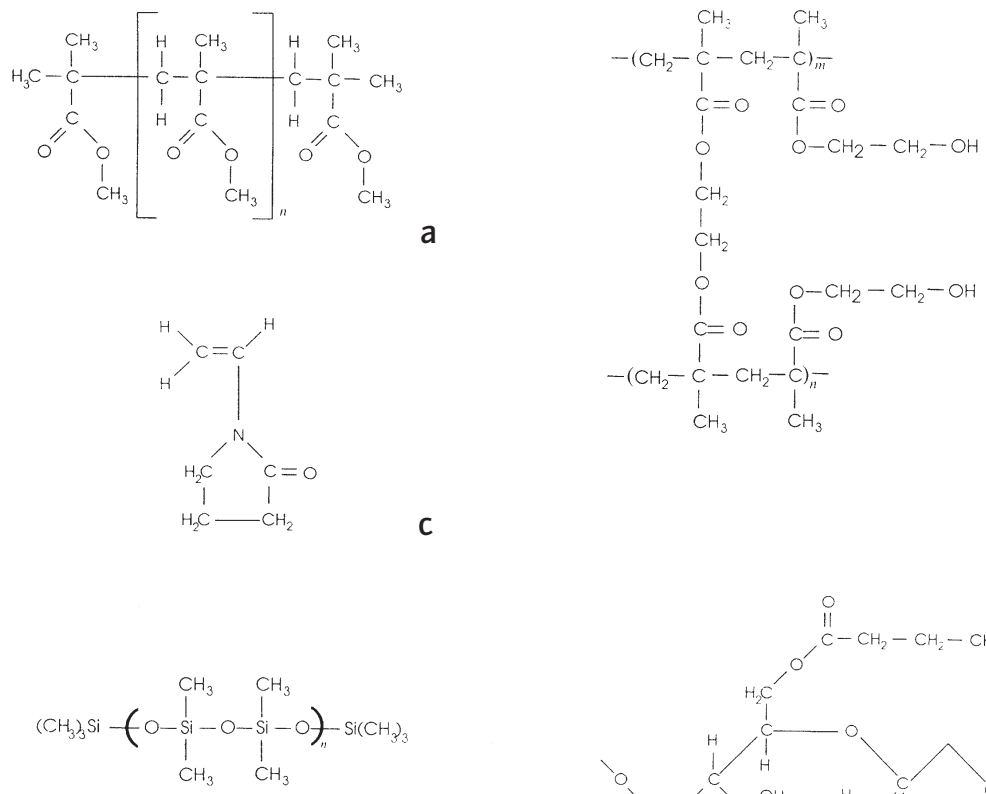

d
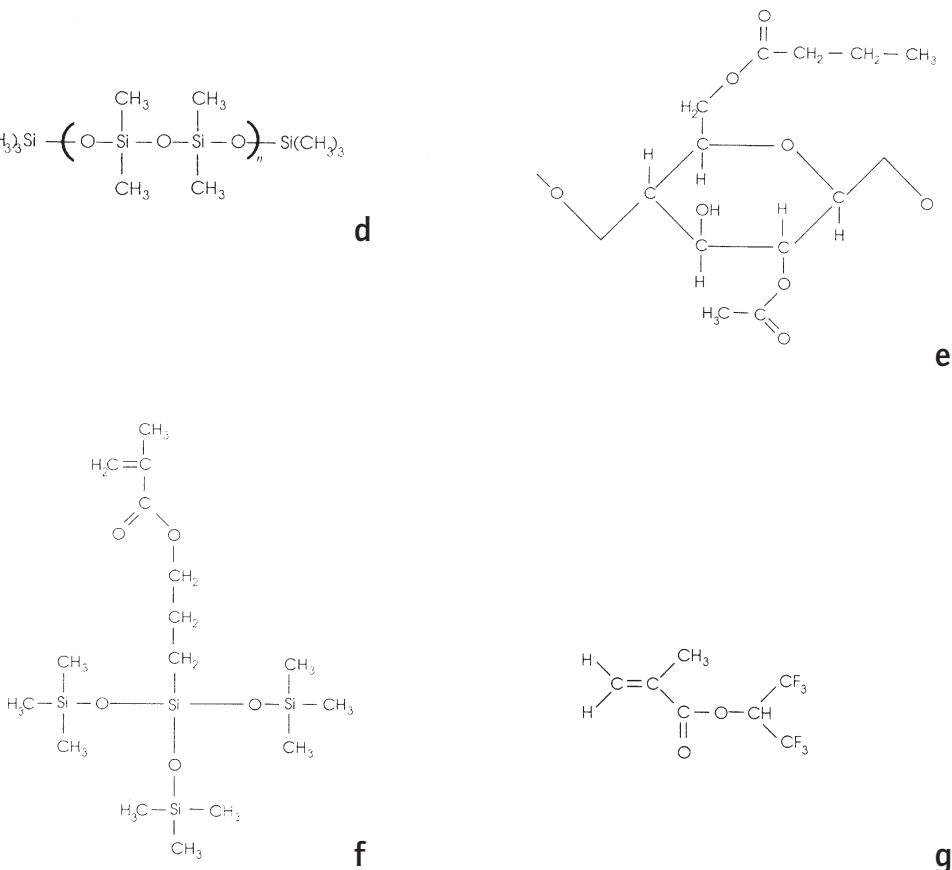

Figure 4. Chemical structure of various polymers in use for contact lenses.

(a). Polymethyl methacrylate (PMMA). (b). Crosslinked Poly (2-hydroxyethyl methacrylate) (PHEMA). Note the difference of a $\mathrm{CH} 3$ end group in PMMA (fig. 4a) and an $\mathrm{OH}$ end group in HEMA making it a hydrophilic gel. (c). Nvinyl-pyrrolidone (NVP). (d). Silicone rubber, poly(dimethylsiloxane). (e). Cellulose acetate butyrate (CAB). (f). Example of a component for SAM copolymers, Methylcryloxypropyl tris(trimethyl-siloxy)silane (TRIS). (g). Example of a component for FASAM copolymers, Hexafluoro-2-propyl methacrylate. 
PMMA CLs that were called "flexi" CLs and gas permeable CLs "semi-soft". The manufacturing of PMMA CLs has traditionally been lathe cutting and polishing on a spindle. Some manufacturers used semi-finished products produced by moulding or heat pressing techniques.

HEMA (Fig. 4b) was developed in the 1960s by changing the methyl $\left(\mathrm{CH}_{3}\right)$ end group in the PMMA polymer via a chain of $\mathrm{CH}_{2}$ into an $\mathrm{OH}$ end group. With this modification the material becomes hydrophilic, soft, flexible with some permeability to gasses. Manipulating the amount of modified chains makes it possible to vary the hydrophilic properties and gas permeability of the materials. However, the counterpart of this can be a loss in material strength and an increase in bounding of elements from the tear film.

HEMA co-polymers (Fig. 4c) have been developed in attempts to meet the demands of biocompatibility with the eye and to increase gas permeability. The principle difference with simply adding more $\mathrm{OH}$ end groups to HEMA is that cross linking chains with entirely different composition are added to the polymer. In this way one can manipulate water uptake and ion-binding properties. Today the FDA classification of hydrophilic materials into four groups is generally accepted (low/high water content and ionic/non-ionic, Table 1). SCLs are today produced by lathe cutting, spin casting or cast / compression moulding. The first production type is used to produce special CLs, the others in mass production of standard CLs.

Rigid gas permeable (RGP, Figs. 4e-g) is a term that has been introduced to differentiate from the classical "hard" PMMA and "soft" PHEMA CL materials. The actual reason to develop RGP materials was to combine stability properties as in PMMA with permeability properties as in PHEMA materials. The first materials that had some permeability to gasses were introduced 30 years ago. However, the materials needed a better stability, wettability, and oxygen permeability in relation to thickness and lens design in order to make these materials suitable for prescribing on a large scale. This resulted in the introduction of the siloxanyl alkyl methacrylate (SAM, Fig. 4f) and fluoro/siloxanylalkyl methacrylate (FASAM, Fig. 4g) copolymers in the 1980s. The incorporation of silicon into the polymer results in an increase of oxygen permeability, whereas fluorine not only enhances oxygen permeability but also rigidity, stability and deposit resistance. Consequently these materials were called high gas permeable (HGP). Although oxygen permeability is generally used as the main term in relation to corneal physiology, the permeability for other gasses (e.g. carbon dioxide as a waste product of aerobic corneal metabolism) is in some situations of importance. $38,44,132$

Classification of CL materials in relation to gas permeability has changed over the years not only due to the constant development of the materials but also due to research and debate on the correct method to measure this property. ${ }^{132}$ Recently new standards for classification of CL materials and oxygen permeability have been recommended by EN ISO 11539 (Table 2). 133,134

The clinical experiments with early HGP materials showed many problems with deposit formation and deformation. These materials also showed difficulties in machinability if pro- 


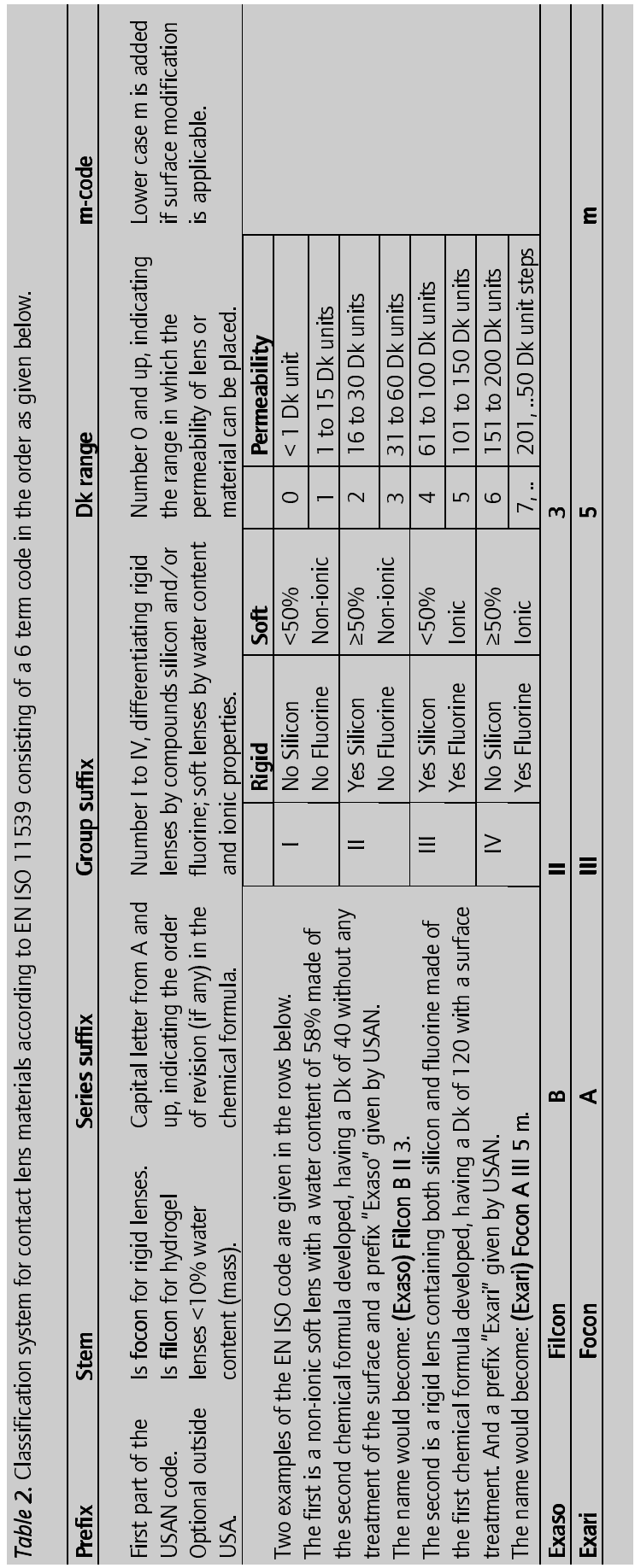


duced using classical lathe cutting methods. Therefore, HGP CLs are not generally accepted for normal correction of ametropia under daily wear conditions. However, the technique has evolved and the use of semi-finished pieces of material (button) and the now widely used Computer Numeric Controlled (CNC) manufacturing procedure raises the question whether the hesitation to prescribe the physiologically more favourable HGP CLs is still valid.

Since 1970 SILICON rubber (Fig. 4d) has been used as a material for CLs. The favourable properties of this material are its high oxygen permeability relatively independent of the thickness and the combination of softness and stability. However, since the nature of the material is hydrophobic, a surface treatment is necessary to make it biocompatible with the tear film. Experiments with Silicon CLs to correct for keratoconus ${ }^{135}$ and aphakia ${ }^{136}$ have in adults revealed problems due to heavy adherence of deposits from the tear film. In contrast, we and others 137,138 have seen rather good results with these CLs if applied to infants that became aphakic after surgery for congenital cataract. Therefore, it is worthwhile investigating the long-term results of a systematic approach for the permanent wearing of silicon CLs in very young children with aphakia after surgery for cataracts (chapter 2.2).

Investigating the clinical and practical use of HGP contact lenses in daily wear has been the basis for our research, as described in chapter 3 of this thesis.

Geometry and fitting

The Total Diameter (TD) of the CL determines the area of the eye that is covered by the lens in situ (Fig. 5). The first CLs with a TD between 18 to $24 \mathrm{~mm}$ are therefore called scleral contact lenses. Small lenses with a TD of 8 to $12.5 \mathrm{~mm}$ are called corneal contact lenses and lenses with a diameter from 12.5 to $18 \mathrm{~mm}$ semi-scleral contact lenses.

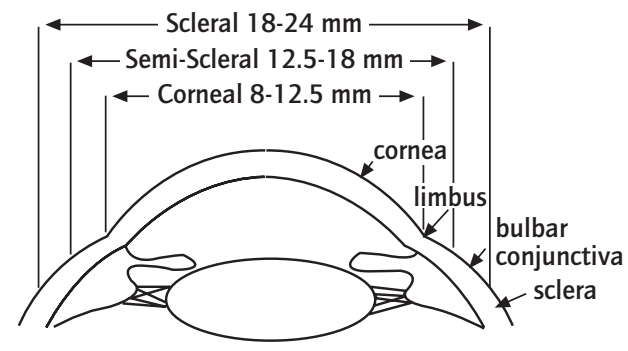

Figure 5. Contact lenses with various diameters in relation to the eye dimensions.

The back surface, also called posterior surface of a CL can be constructed with a single spherical shape. Such a CL is rotationally symmetric and has a spherical mono-curve design. If the cornea has a similar shape, the radius of curvature of the CL (base curve) should be taken as that measured with a device called keratometer (keratos is Greek for cornea). In CL terminology this is referred to as: 'take the base curve of the CL on-K'. Because it has long been recognised that the cornea flattens toward the periphery, this type of CL can only fit the cornea if 
the diameter is very small. With larger diameters the shape volume (sagittal depth) of the CL becomes larger than that of the cornea on the same diameter. Consequently the periphery of the CL would come into contact with the cornea (steep fit). 139 With increasing the radius of curvature of the CL one could solve the problem in the periphery, but finally the central part of the CL would come into contact with the cornea (flat fit). ${ }^{140}$ The classical solution to this problem has been described by Bier ${ }^{141}$ introducing the "Contour" technique in which changing the radius of curvature at a certain distance from the centre of the $\mathrm{CL}$ (bi-curve) allows for better alignment to the natural corneal shape. Taking this concept further to optimise corneal alignment has led to a set of classical CL designs called tri-, tetra-, or multi-curves in which the boundaries of the curves (transitions) are smoothened by polishing (blending). Modern posterior CL geometry involves gradual changes in radius of curvature from centre to periphery without transitions. These designs are usually simply called aspheric. 83,142 The term aspheric can lead to confusion because it could mean deviation from a circular shape not only in the meridian but also in the rotational shape. It would be more correct to classify the CL as described above as being rotationally symmetric with a posterior meridional aspheric design. As described earlier, the cornea usually flattens towards the periphery. In that sense it is aspherical, taking a cross-section over the meridians. Confusingly, the cornea also shows usually different shapes per meridian, meaning that it is not rotationally symmetric. A normal cornea has a longer radius of curvature in the horizontal meridian (flatter) than in the vertical (steeper). ${ }^{143}$ Such a surface has two principal meridians lying 90 degrees from each other and is called toric. Optically this situation leads to astigmatism referred to as 'with the rule corneal astigmatism'. Some corneas show the opposite (against the rule astigmatism). Moreover, the principle meridians can deviate from horizontal and vertical but remain 90 degrees from each other (oblique astigmatism) or the radius of curvature varies over the surface without having two principle meridians (irregular astigmatism). If the difference in radius of curvature between meridians of the cornea is small, a rotational symmetric (although aspherical in 2-D design) can fit the eye. Large differences in corneal radii among meridians but lying 90 degrees from each other would call for $\mathrm{CL}$ posterior surfaces with a toric design (inside toric). However, the cornea does usually not even fit a pure toric description. ${ }^{143,144,145}$ Therefore, modern research involves the possibility of designing and producing CLs that follow corneal shape in all dimensions to obtain so called Contact Lenses with Aspheric Asymmetric Surfaces (CLAAS). ${ }^{146}$ A feasibility study about the possibilities of using soft CLAAS to correct the eye in keratoconus is presented in chapter 4.5 .

\section{Optical correction}

By replacing the optical power of the cornea, the anterior surface of a CL becomes the main surface responsible for the optical correction of the eye. Similar to the posterior CL surface there is confusion about the term aspherical in relation with meridional and rotational domains. In the meridional domain the radius of curvature of the front surface of a CL should be such that optical correction of the ametropic eye is reached in all meridians. In with the rule astigmatism, usually the posterior and anterior curvatures of a rigid CL can be taken to be rotationally symmetric (spherical in the rotational domain). Correction of the corneal astigmatism takes place by the toricity of the tear layer with the CL in situ. If the total astigmatism of the 
eye does not equal corneal astigmatism some residual astigmatism will remain. In this case the anterior surface of the CL should contain the correction for this residual astigmatism (front toric). With a rotationally symmetric posterior surface the CL may rotate on the eye resulting in poor alignment of the meridional powers of $\mathrm{CL}$ and eye. Therefore, methods of CL stabilisation are necessary. 51,147

Applying a thin SCL on an astigmatic eye can, due to lens flexure, only result in an average correction of the ametropia (sphere equivalent correction). Correction of the astigmatic part of the ametropia can be reached by the use of a toric SCL incorporating a way of stabilisation. Finding the right parameters of such a CL is difficult because many parameters of the eye and the CL interfere. Traditionally one uses a trial and error method by observing the behaviour of various so-called trial or diagnostic CLs. It would be more efficient and beneficial for both patient and practitioner if the parameters for a soft toric CL could be based on a custom-made procedure. It is remarkable in this respect that in some countries the use of trial lens fitting sets has been restricted for safety reasons.

Research on the aspects of optimising fitting procedures for HGP and soft toric CLs avoiding trial lens fitting are covered in chapters 3 and 4 of this thesis, respectively.

\section{Indications}

The indications for prescribing CLs can be divided in four major groups each containing use in specific diagnoses. The first group covers the correction of refractive error and accommodation deficiency of normal healthy eyes. This group is often referred to as "cosmetic", since the motivation for most patients to wear CLs is a hesitation to spectacles. However, in the English terminology the term cosmetic CLs also means that the CL is improving the appearance of the eye (group 3). The main feature of using CLs compared to spectacle lenses is that the correction is placed nearer to the principle optical planes of the eye. The benefits are largely reduced effects of magnification, minor prismatic effects under different viewing angles and less distortion in the periphery of the visual field.

A second group of CL indications is formed by diagnoses in which optical deformation of the cornea is present, usually after pathology, trauma, accompanying corneal dystrophy or today also post-refractive surgery. In these cases the CLs provide an optically smooth anterior surface. The corneal irregularities are greatly reduced by the tear layer between the posterior surface of the $\mathrm{CL}$ and the irregular cornea. However, caution is needed with respect to the development of adverse effects in these pathological conditions.

Indication group 3 contains CLs used to correct iris or pupil defects, to provide occlusion, and to improve cosmetically the appearance of the eye after pathology / trauma, but also because people like to change the colour of their eyes (fun-lenses).

A fourth major group are CLs used as an aid to therapeutic actions. Paediatric CLs are used in cases of high myopia and aphakia after congenital or juvenile cataract in order to provide permanent optical correction necessary to stimulate the visual development in infants. The use of SCLs has become very important as a bandage to support healing of the corneal epithelium after pathology and trauma. Further, the hydrophilic properties of SCLs are used as a reservoir for (artificial) tears in cases of dry eye or as a drug-release container. Large scleral SCLs are used as regulator of a filtering bleb after glaucoma surgery. 55 


\section{Assessment of contact lens related influence on the physiology of the eye}

With the development of better materials, advanced manufacturing methods and more precise fitting techniques, the influence a CL might have on the physiology of the eye has gradually declined over the years. On the other hand, research has shown that a CL without any noticeable influence is an utopia. A CL remains principally a "corpus alienum" (foreign body) to the eye. Today there is no doubt that under strictly controlled circumstances the wearing of modern CLs is relatively safe. However, a large proportion of the CL literature is concerned with adverse reactions, found retrospectively and ranging from slight foreign body sensation during adaptation to serious complications as neo-vascularisation, infiltrates and microbial keratitis. Contrary to techniques like refractive surgery and intra ocular lens implantation, the application of CLs has the advantage of being non-invasive. If there is a problem, the patient or the practitioner can simply discontinue wearing them. However, there are two pitfalls here. One is that adverse effects can proceed gradually and the wearer might become accustomed to the concomitant symptoms. The other is that for a practitioner it is impossible to control completely the wearing habits and most important the care regime of the patient. In general CL patients are strongly motivated to wear the lenses but compliance to proper care and handling is low. Basic rules to safe CL wear are, therefore, thorough patient instructions and regular follow-up examinations by a well-trained CL practitioner. ${ }^{148}$

The influences CLs might have on the physiology of the eye have been thoroughly investigated and can be categorised as: mechanical (fit of the CL), metabolic (oxygenation), conditional (CL and environment) and temporal (wearing scheme). Comprehensive descriptions of these influences with related signs, symptoms and management can be found in most textbooks (e.g. in Phillips and Stone ${ }^{149}$ chapter 19-20 and Mackie ${ }^{123}$ chapter 12-13).

Figure 6 provides a general summary of the possible influences that rigid and soft CLs might

\begin{tabular}{|c|c|c|c|}
\hline Action & $\begin{array}{l}\text { Severity of adverse effect } \\
\text { Acceptable } \\
\text { Next follow up }\end{array}$ & $\begin{array}{l}\text { Abnormal } \\
\text { Change CL }\end{array}$ & $\begin{array}{l}\longrightarrow \\
\text { Pathological } \\
\text { Refrain wear }\end{array}$ \\
\hline Staining & Transient stippling & Punctata & Erosion \\
\hline Visual Acuity, decrease & $<0.1$ & & $>0.3$ \\
\hline K-Readings, change & $<0.1 \mathrm{~mm}$ & $>0.1 \mathrm{~mm}$ & $>0.3 \mathrm{~mm}$ \\
\hline Ametropia, change & $<1.00 \mathrm{D}$ & \multirow{2}{*}{$\begin{array}{l}>1.00 \mathrm{D} \\
>7 \%\end{array}$} & $>3.00 \mathrm{D}$ \\
\hline Corneal thickness, increase & $<7 \%$ & & $>25 \%$ \\
\hline Epithelial microcysts & $<10$ & $>10$ & $>20$ \\
\hline Descemet layer & Transient blebs & $>2$ striae, 1 fold & Crossed striae \\
\hline Endothelial polymegethism* & 1 grade increase & $>1$ grade increase & $>2$ grades increase \\
\hline Limbal vascularisation & $<0.5 \mathrm{~mm}$ & $>0.5 \mathrm{~mm}$ & Neo-vascularisation \\
\hline Oedema RGP & $\mathrm{CCC}<8 \mathrm{~min}$ & $\mathrm{CCC}>8 \mathrm{~min}$ & Persistent local \\
\hline Oedema Soft & \multicolumn{3}{|c|}{ Difficult to detect --_en $\quad$ Control vacuum } \\
\hline Symptoms & & $\begin{array}{l}\text { Tear h } \\
\text { Oeden } \\
\text { Hyper } \\
\text { Pain } \\
\text { Photo } \\
\text { Halo's }\end{array}$ & $\begin{array}{l}\text { Tear hypersecretion } \\
\text { Oedema eye lids } \\
\text { Hyperaemia conjunctiva } \\
\text { Pain } \\
\text { Photophobia } \\
\text { Halo's }\end{array}$ \\
\hline Secondary pathology & & & $\begin{array}{l}\text { Corneal irregularities } \\
\text { Keratitis } \\
\text { Ulcer }\end{array}$ \\
\hline
\end{tabular}

$C C C=$ central corneal clouding. ${ }^{*}$ As evaluated using the CCLRU ${ }^{175}$ grading scale.

Figure 6. Scheme of possible adverse effects that rigid and soft contact lenses can have on the physiology of the anterior segment of the eye. Note the difficult to detect oedema in soft contact lenses which represents a control vacuum between acceptable and pathological conditions. 
have on the eye. 150,175 The scheme includes descriptions of signs and symptoms relative to acceptable, abnormal and potentially pathological conditions. For rigid CLs these typically involve an oedematous influence known as central corneal clouding (CCC) and various staining patterns of the corneal epithelium. Mandell ${ }^{151}$ described how CCC can be detected using the method of sclerotic scatter with the slit lamp. CCC can be classified as an accepted adverse effect if no longer present 8 minutes after removal of the CL. This represents an example of the border between acceptable and abnormal influence in the scheme of Fig. 6. In the same way other influences of rigid CLs (depending on gas permeability) are changes in Snellen visual acuity 152-154, K-readings and corneal topography (corneal warpage) ${ }^{17,155-157 \text {, }}$ ametropia ${ }^{152,158,159}$ and the influences as can be assessed by observing staining of the cornea after the application of fluorescein or Rose bengal. ${ }^{160-166}$ The influences of rigid CLs at the borders of acceptable and abnormal can be easily assessed using routine clinical ophthalmic procedures.

For SCLs the situation is entirely different. Mechanical staining is seldom; reported staining involves overall corneal staining ${ }^{167}$ and typically arcuate staining ${ }^{168}$ and desiccation staining. ${ }^{166}$ Changes in visual acuity, K-readings or ametropia remain minor unless the influence is already at the border of possible pathology. ${ }^{169,170}$ Because of the full corneal coverage, oedema in SCLs is difficult to detect unless it becomes severe. ${ }^{171,172}$

As listed in Fig. 6, one can see that the patient would report symptoms only if the influence is already in the higher segment of the abnormal influence. This phenomenon is called the control vacuum in SCLs. The danger is that influences become gradually worse and can pass the border of irreversible changes like persistent oedema, corneal irregularities and neo-vascularisation. The greatest danger lies in the fact that the normal defence mechanisms of the eye are weakened. Especially in combination with permanent or extended wear of SCLs, poor condition of the CLs or poor disinfecting care by the patient this can result in serious complications..$^{50}$ Already in the 1970s and 1980s a tremendous amount of research by means of controlled oxygenation studies was done $36-38,41-43,173$ in order to find ways of eliminating the control vacuum in SCLs by assessment of corneal thickness changes and slit lamp observation of the corneal stroma and endothelium. From this research it could be deduced that the border of normal physiological influence of a CL on the cornea is reached with an increase in central corneal thickness of $7 \%$, and/or the observation of striae at the Descemet layer, and/or the presents of microcysts or vacuoles. In the case of the application of SCLs these influences seem to progress gradually and in relation with degradation of the condition of the CL. A positive factor to prevent serious adverse reactions is the frequent replacement of SCLs or ideally the use of daily disposable SCLs.

Despite the positive developments in contact lens technology as described above, patient compliance and stringent follow-up by well-educated CL practitioners seem to remain important to guarantee safe wear of CLs. In a recent report from the Health Council of the Netherlands ${ }^{174}$, the committee that had investigated possible health risks associated with wearing contact lenses concluded that such risks are present and that extended wear lenses have the highest risk. However, the committee found it reasonable to assume that the risks are diminished by correct fitting, proper instruction and regular check-ups. Furthermore, it noted that there is legislation on the quality of contact lenses but not on fitting, instruction and check- 
ups. Also, there is no centralised record of the complications due to contact lens wear. It is interesting that the committee felt that the availability of contact lenses from sources other than optometrists or ophthalmologists could increase the risk of complications. However, there are no data available to predict the magnitude of that risk, and there is no foolproof system available to enforce regulation rules for the distribution of contact lenses. The committee recommended that fitting of contact lenses and checking wearers' eyes should be done by appropriately trained experts (optometrists and ophthalmologists). The committee held that the incidence of complications could be reduced if the professionals involved developed protocols for the fitting, correct instruction, good aftercare and supervision of contact lens wear. Within this thesis, schemes for fitting are developed and tested using objective schemes to assess the influence of CLs on eye physiology 175 (chapters 3 and 4). The results of these investigations could be of use in composing the recommended professional protocols.

\section{Measuring techniques}

Simultaneously with the development of more precisely defined CL shapes, there has been an impressive improvement in techniques to measure the dimensions of the front surface of the eye. The first approach was to consider the corneal surface to be spherical. A spherical surface can be measured by comparing the size of a reflected image from the cornea (first Purkinje image) with the size of the original image. This was the basis on which the keratometer (originally called ophthalmometer) was developed by Javal in 1889 . If the relative location of the image is known, the parameter by which a spherical surface can be described, the radius of curvature, can be estimated (Fig. 7a). In a keratometer the object is actually formed by two separated objects (usually called mires) placed at a certain distance from the optical axis of the device. In this way it is not the size of an object that is compared with a formed image, but the separation between the two mires at the object plane is compared with the measured separation in the image. To measure exactly the latter separation, keratometers have a built-in doubling device. As can be seen from the optical construction of the image in Fig. 7a, only a very small area of the cornea is used to reflect each mire (depending on the instrument and radius of the cornea, from 0.1 to $0.8 \mathrm{~mm}$ ). It can also be seen that the keratometer actually presents an average measurement using two of these very small areas that are separated from each other (depending on the device and the radius of the cornea, from 2.0 to $3.5 \mathrm{~mm}$ ). To decrease inaccuracies because of this and also to reduce the inherent problem that the object is not placed at infinity and thus the image is not formed exactly at the focal point of the cornea, Mandell176 developed keratometers with long working distance and small objects (small mirror keratometry). With these devices he performed measurements of the peripheral corneal curvature ${ }^{177}$ by having the patient fixate a movable off-axis light source. The need for extra information on the corneal periphery can be easily deduced from Fig. 5, if one realises that the smallest CL has a diameter of $8 \mathrm{~mm}$ and classical keratometry only provides information on an area of around $3.0 \mathrm{~mm}$. Using of an off-axis fixation device Wilms ${ }^{76}$ developed a method that can be used with most ordinary keratometers and delivers an estimation of E-values at 30 or 25 degrees from the central axis (Fig. $7 \mathrm{~b})$. The resulting sagittal topometric values can in principle be used to compute parameters of the posterior CL surface. It is important to investigate to what degree the extra information from this method can contribute to precision and efficiency of CL fitting. 


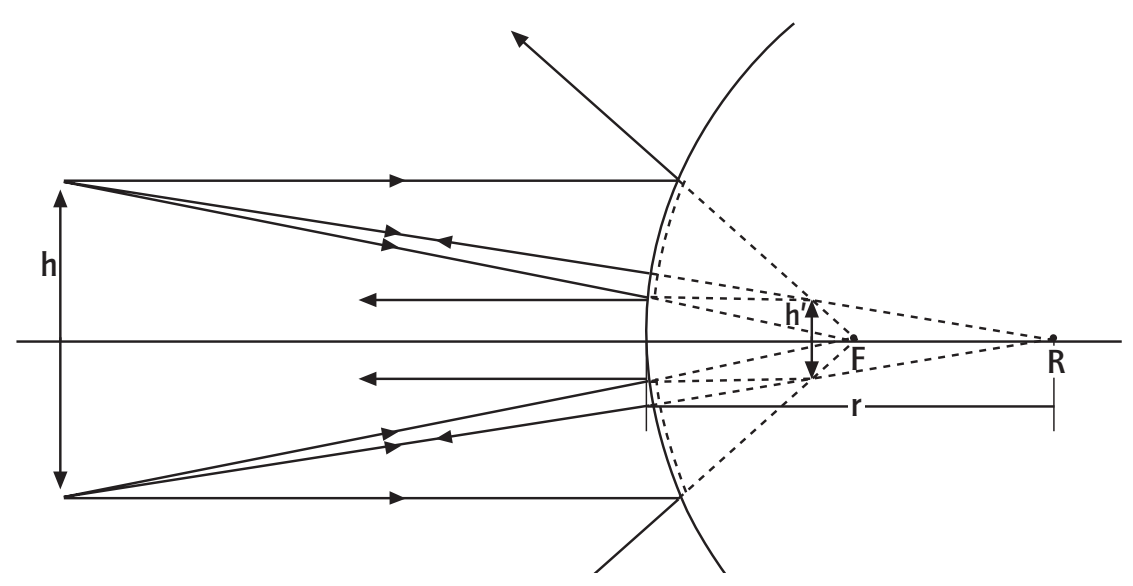

Figure 7a. The use of the keratometer in contact lens fitting.

The principle of keratometry is based on image formation as with a convex mirror. The radius of curvature of the cornea $(r)$ determines the difference in distance between the object mires $(h)$ and the same at the reflected image side $\left(h^{\prime}\right)$. Note that only two small and separated areas (dashed parts) of the cornea actually contribute to the measurement. Note also, that the longer the distance between the object mires (h) and the cornea is, the closer $\mathrm{h}^{\prime}$ is located to the focal plane of the cornea.

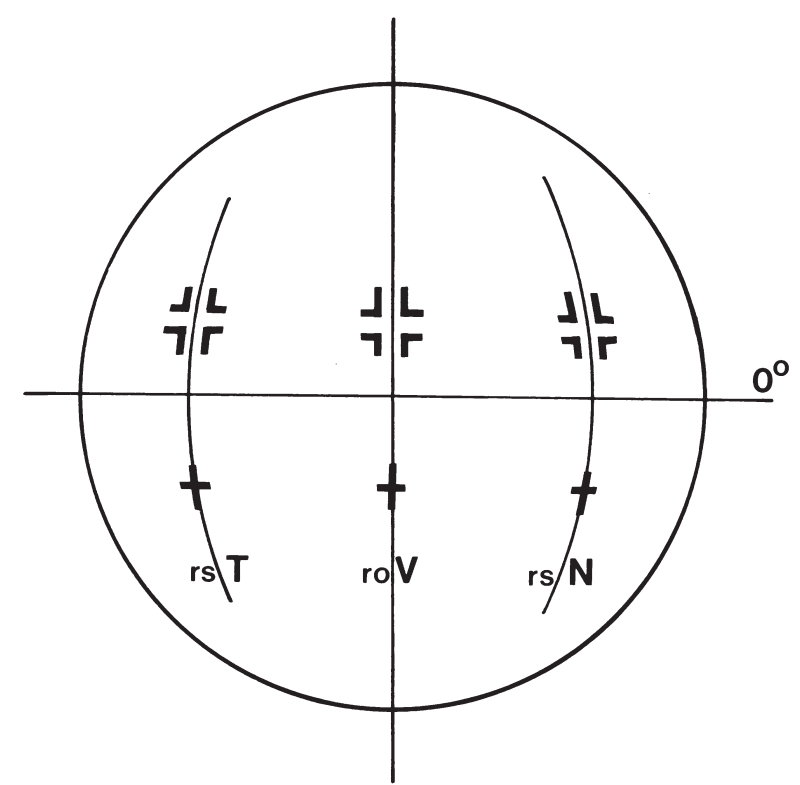

Figure $7 \mathrm{~b}$. The areas measured using sagittal topometry as proposed by Wilms. Note that the horizontal meridian is measured in the periphery with the keratometer mires in a vertical position. In the computation the outcome of this measurement is compensated by the central astigmatism to arrive at the estimated peripheral radius in the horizontal meridian. 
With concentric rings printed on a disk with a central hole, Placido 178 (Placido disk) made it possible to qualitatively evaluate corneal irregularities and astigmatism (Fig. 8a). Gullstrand ${ }^{179}$ took a major step in corneal topography by placing a photographic camera in the central hole and by measuring the sizes of the rings estimated the corneal radii over a still limited but definitely larger area of the cornea than can be measured using the keratometer. However, when the surface is irregular as is often the case with pathological corneal surfaces, reliable reconstruction of the entire surface becomes impossible (Fig. 8b).
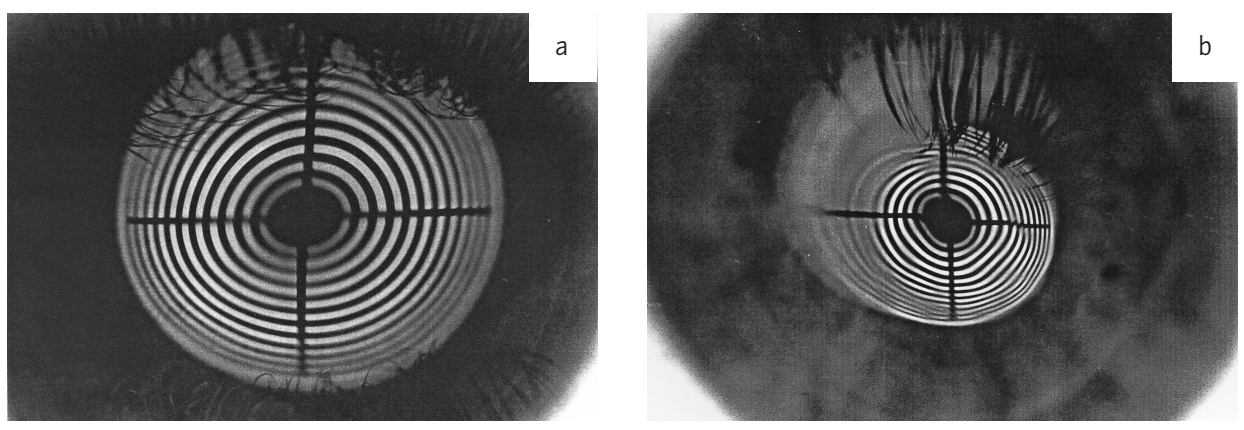

Figure 8. The use of the Placido disk in contact lens fitting.

(a). Photographic image of a Placido disk reflected on an astigmatic cornea.

(b). Placido disk photograph from a cornea with a very steep apex, a flat superior part, and a steep inferior part (keratoconus). Note the very small area of the cornea that can (due to the steep radius) be imaged and that cannot be interpreted usually or only with severe error.

With the development of small and relatively cheap Charge Coupled Device (CCD) sensors and the introduction of Personal Computers, the old camera system of Gullstrand has been transformed into modern Computer-Assisted Video Keratometers (CAVK). With these devices, topography of the cornea has become a widely used clinical tool. The inherent limitations of imaging by specular reflection (Fig. 8) led to the development of alternatives. Ambiguities can be avoided when the surface itself is measured rather than a derivative. ${ }^{82} \mathrm{~A}$ device that measures the shape of the whole anterior eye, rather than the axial or local radius of curvature, is the Maastricht Shape Topographer (MST). Advantageous to the design of scleral and soft CLs, the MST is able to present a profile cross-section of the entire front surface of the eye including the limbal area. ${ }^{81}$

The size and position of the pupil is an important element in the optical system of the eye. With modern possibilities to precisely design CL optics, pupillometry has become an important issue. The availability of an easy-to-use and reliable method to measure pupil size and position under controlled conditions is essential to further explore optical design of multifocal and non-rotationally symmetric CLs.

In ophthalmic practice the use of fluorescein is well known. Relatively new is the use of fluorescein in shape measurements of the eye and the possibilities to enhance the visibility of specific eye conditions. To optimise these techniques it is important to further study fluorescence phenomena in relation to excitation light, type of fluorescein, concentration and substrate. 


\subsection{Aims of the studies}

The aims of the studies as described in this thesis were:

1 To use recent developments in technology in order to design new contact lens geometries, fitting methods, and measuring techniques.

2. To test the clinical application of newly developed contact lens materials, designs, fitting procedures and care products.

3. To provide practical rules for the application of newly developed contact lenses.

\subsection{Outline of this thesis}

Chapter 1 gives a general introduction to the subject and describes the aims of this thesis.

In Chapter $\mathbf{2}$ the clinical application of silicone contact lenses of high plus power in young aphakic children (2.2) and coloured soft contact lenses as an artificial iris (2.3) are described.

Chapter 3 is dedicated to the fitting and care of HGP contact lenses. This chapter covers HGP materials, design and fitting (3.2), and HGP lens selection and care systems (3.3).

In Chapter $\mathbf{4}$ special optical applications with soft contact lenses are presented. It involves the clinical application of a custom-made toric soft contact lens system (4.2), varifocal soft contact lenses (4.3), aspheric soft contact lenses in high ametropia (4.4) and a feasibility study on the possibilities to correct keratoconus with specially designed soft contact lenses (4.5).

Chapter 5 covers some aspects of measuring the eye presented in sections on height topography of the anterior eye surface (5.2), pupillometry (5.3) and the visibility of the tear film and underlying tissues using fluorescein (5.4).

Finally, the results of the studies are summarised and put into broader perspective. 


\section{References}

1. Hills J. A review of the literature on primary communications in science and technology. London, Aslib, 1972.

2. Groot de A. The theory of the science forum: Subject and purpose, Groningen, Psychologische Instituten R.U., 1983.

3. Holden B. Preface. Silicone Hydrogels, the rebirth of continuous wear contact lenses. Oxford, Butterworth-Heinemann, 2000. ISBN 0750644621, edited by D. Sweeney.

4. Vinci da L. Codex of the Eye, Manuscript D, 1508.

5. Calder R. Leonardo and the age of the eye. London, Heinemann, 1970.

6. Descartes R. Discourse de la Methode, Discourse No. 7. La Dioptrique 1637:79.

7. Enoch JM. Descartes' Contact Lens. Am J Optom Arch Am Acad Optom 1956;33:77-85.

8. Obrig AB, Salvatori PL. The History and Development of Contact Lenses. Contact Lenses. Ed. 3; Chapter 5:125-27. New York, Obrig Laboratories Inc., 1957.

9. Fick AE. Eine Kontaktbrille. Arch f Augenheilk 1888:279-89.

10. Panas P. Presentation of instruments and apparatus: A discussion of Kalt's use of shells of glass against keratoconus. Bull Acad Med 1888;3:400-1.

11. Mueller A. Brillenglaeser und Hornhautlinsen. Medical faculty, Kiel, Universitaet Kiel, 1889.

12. Rohr von M, Boegehold H. Das Brillenglas als Optisches Instrument Ed. 17. Berlin, Springer, 1934.

13. Farbenindustrie A. Method of adhering lenses and contact lenses of polymerization products. Patent, Germany, 1935.

14. Feinbloom W. A Plastic Contact Lens. Am J Optom Arch Am Acad Optom 1937;14:41-9.

15. Nugent M. The corneal lens, a preliminary report. Ann West Med Surg 1948;2:241.

16. Hartstein J. Corneal warping due to wearing of corneal contact lenses. A report of 12 cases. Am J Ophthalmol 1965;60:1103-4.

17. Pratt Johnson JA, Warner DM. Contact lenses and corneal curvature changes. Am J Ophthalmol $1965 ; 60: 852-5$.

18. Ruben M. The fitting of corneal contact lenses. Trans Ophthalmol Soc U K 1967;87:661-70.

19. Ruben M. Corneal changes in contact lens wear. Trans Ophthalmol Soc U K 1967;87:27-43.

20. Sarver MD, Harris MG. Corneal lenses and "spectacle blur". Am J Optom Arch Am Acad Optom 1967;44:316-8.

21. Mandell RB, Harris MG. Theory of the contact lens adaptation process. J Am Optom Assoc 1968;39:260-1.

22. Millodot M, Larson W. New measurements of corneal sensitivity: a preliminary report. Am J Optom Arch Am Acad Optom 1969;46:261-5.

23. Boyd HH. Evaluation of 1,000 patients wearing small thin contact lenses. Nippon Kontakuto Renzu Gakkai Kaiin Dayori 1969;11:13-5.

24. Wichterle O, Lim D. Hydrophilic gels for biological use. Nature 1960;185:117-8.

25. Gasson A. Soft Lens Fitting. Contact Lenses: A textbook for practtioner and student. Ed. 2;Vol. 2, Chapter 14:400-1. Edited by Stone J, Phillips AJ. London, Butterworths, 1981.

26. Wichterle O, Lim D. Method for producing shaped articles from three dimensional hydrophilic high polymers. Patent, USA, 1968.

27. Mandell RB. Corneal edema from hard and hydrogel contact lenses. J Am Optom Assoc 1976;47;3:287. 
28. Harris MG, Sarver MD, Polse KA. Corneal curvature and refractive error changes associated with wearing hydrogel contact lenses. Am J Optom Physiol Opt 1975;52:313-9.

29. Sarver MD, Polse KA, Harris MG. Results with the Bausch and Lomb F3 series Soflens contact lens. Am J Optom Physiol Opt 1977;54:67-9.

30. Rofojo MF. Oxygen permeability of contact lenses [letter]. Am J Ophthalmol 1980;89:881.

31. Orsborn GN, Zantos SG. Corneal desiccation staining with thin high water content contact lenses. CLAO J 1988;14:81-5.

32. Wright P, Mackie I. Preservative-related problems in soft contact lens wearers. Trans Ophthalmol Soc U K 1982;102:3-6.

33. Mackie IA. Adverse Reactions to Soft Contact Lenses. Medical Contact Lens Practice: a systematic approach. Chapter 13:124-63. Oxford, Butterworths-Heinemann, 1993.

34. Hill RM, Leighton AJ. Physiological time courses associated with contact lenses. Temperature. 1. Animal time courses with scleral lenses. Am J Optom Arch Am Acad Optom 1963;40:427-38.

35. Hill RM. Oxygen uptake of the cornea following contact lens removal. J Am Optom Assoc 1965;36:913-5.

36. Polse KA, Mandell RB. Hyperbaric oxygen effect on corneal edema caused by a contact lens. Am J Optom Arch Am Acad Optom 1971;48:197-200.

37. Polse KA, Sarver MD, Harris MG. Corneal edema and vertical striae accompanying the wearing of hydrogel lenses. Am J Optom Physiol Opt 1975;52:185-91.

38. Hill RM. The physiology of contact lens systems. J Am Optom Assoc 1976;47;3:284.

39. Polse KA. Etiology of corneal sensitivity changes accompanying contact lens wear. Invest Ophthalmol Vis Sci 1978;17:1202-6.

40. Hill RM, Andrasko G. Oxygen and water. J Am Optom Assoc 1981;52:225-6.

41. Holden BA, Mertz GW, McNally JJ. Corneal swelling response to contact lenses worn under extended wear conditions. Invest Ophthalmol Vis Sci 1983;24:218-26.

42. Holden BA, Mertz GW. Critical oxygen levels to avoid corneal edema for daily and extended wear contact lenses. Invest Ophthalmol Vis Sci 1984;25:1161-7.

43. Holden BA, Williams L, Zantos SG. The etiology of transient endothelial changes in the human cornea. Invest Ophthalmol Vis Sci 1985;26:1354-9.

44. Polse KA. Gas-permeable lens materials and designs. Int Ophthalmol Clin 1986;26:131-48.

45. Brennan NA, Efron N, Holden BA, Fatt I. A review of the theoretical concepts, measurement systems and application of contact lens oxygen permeability. Ophthalmic Physiol Opt 1987;7:485-90.

46. Tighe BJ. Contact lens materials. Contact Lenses: A Textbook for Practitioner and Student. Ed. 3; Chapter 3:72-124. Edited by Phillips AJ, Stone J. London, Butterworth, 1989.

47. Holden BA, Sweeney DF, Vannas A, Nilsson KT, Efron N. Effects of long-term extended contact lens wear on the human cornea. Invest Ophthalmol Vis Sci 1985;26:1489-501.

48. Holden BA. Perspectives on the future of contact lens wear: summary. Cornea 1990;9:S69-71.

49. Poggio EC, Glynn RJ, Schein OD, Seddon JM, Shannon MJ, Scardino VA, Kenyon KR. The incidence of ulcerative keratitis among users of daily-wear and extended-wear soft contact lenses. N Engl J Med 1989;321:779-83.

50. Cheng KH. Contact lens wear and its complications. Thesis, Amsterdam, University of Amsterdam, 1999. ISBN 906464537x

51. Westerhout D. Toric contact lens fitting. Contact Lenses: A Textbook for Practitioner and Student. Ed. 3; Chapter 14:505-54. Edited by Phillips AJ, Stone J. London, Butterworths, 1989. 
52. Carle de JT. Bifocal and multifocal contact lenses. Contact Lenses: A Textbook for Practitioner and Student. Ed. 3; Chapter 16:595-624. Edited by Phillips AJ, Stone J. London, Butterworths, 1989.

53. Port MJA. Cosmetic and prosthetic contact lenses. Contact Lenses: A Textbook for Practitioner and Student. Ed. 3; Chapter 21:789-96. Edited by Phillips AJ, Stone J. London, Butterworths, 1989.

54. Amos DM. The use of soft bandage lenses in corneal disease. Am J Optom Physiol Opt 1975;52:52432.

55. Blok MD, Kok JH, van Mil C, Greve EL, Kijlstra A. Use of the Megasoft Bandage Lens for treatment of complications after trabeculectomy. Am J Ophthalmol 1990;110:264-8.

56. Westerhout D. Soft lenses in other abnormal conditions. Contact Lenses: A Textbook for Practitioner and Student. Ed. 3; Chapter 21:796-817. Edited by Phillips AJ, Stone J. London, Butterworths, 1989.

57. Polmanteer KE. Optical composition of silicone rubber., USA, Dow Corning Corp., 1970.

58. Glasser DB, Weissman BA. Mean oxygen permeability of four flexible contact lens materials. CLAO J 1983;9:29-31.

59. Blackhurst RT. Silicone contact lenses: a personal 13-year perspective. CLAO J 1985;11:57-61.

60. Sweeney D. Silicone Hydrogels, the rebirth of continuous wear contact lenses. Oxford, ButterworthHeinemann, 2000. ISBN 0750644621, edited by D. Sweeney.

61. Long B, Robirds S, Grant T. Six months of in-practice experience with a high Dk Lotrafilcon A soft contact lens. Contact Lens and Anterior Eye 2000;23:112-18.

62. Kamath P. Physical and chemical attributes of an ideal contact lens. Contacto 1969;13:29-34.

63. Stahl N, Reich L, Ivani M. Report on labaroratory studies and preliminary clinical application of a gaspermeable plastic contact lens. J Am Optom Ass 1974;45:302-07.

64. Larke J, Pedley D, Tighe B. Polymers in contact lens applications: Parts 3 and 4. Wettability phenomena. Optician 1973;166:21-30:32-9.

65. Tighe B. The design of polymers for contact lens applications. Br Polymer J 1976;8:71-7.

66. Ng C. Polymers in contact lens applications: Oxygen permeability and surface hydrophilicity of poly(4methylpent-1-ene) and related polymers. Br Polymer J 1976;8:124-30.

67. Giordano GG, Refojo MF. Polymer Chemistry. Contact Lenses, the CLAO Guide to Basic Science and Clinical Practice. Ed. 2; Vol. 1; Chapter 5:73-91. Edited by Kastl P., Kendall/Hunt, 1995.

68. Fonn D, Holden BA. Rigid gas-permeable vs. hydrogel contact lenses for extended wear. Am J Optom Physiol Opt 1988;65:536-44.

69. Schnider CM. Extended-wear RGP contact lenses: a viable alternative to refractive surgery. Optom Clin 1994;4:13-23.

70. Holden B, Swarbrick HA. Extended wear lenses. Contact Lenses: A Textbook for Practitioner and Student. Ed. 3; Chapter 15:580-2. Edited by Phillips AJ, Stone J. London, Butterworths, 1989.

71. Swarbrick HA, Holden BA. Rigid gas permeable lens binding: significance and contributing factors. Am J Optom Physiol Opt 1987;64:815-23.

72. Schnider CM, Terry RL, Holden BA. Effect of lens design on peripheral corneal desiccation. J Am Optom Assoc 1997;68:163-70.

73. Kok JH, Visser R. Treatment of ocular surface disorder and dry eyes with high gas-permeable scleral lenses. Cornea 1992;11:518-22.

74. Pullum KW, Buckley RJ. A study of 530 patients referred for rigid gas permeable scleral contact lens assessment. Cornea 1997;16:612-22.

75. Kok JH, van Mil C. Piggyback lenses in keratoconus. Cornea 1993;12:60-4.

76. Wilms K, Rabbetts R. Practical concepts of corneal topometry. Optician 1977;Sept. 16:7-13. 
77. Wilms K. Ueberlegungen zur Topometrie von Cornea und Kontaktlinsen. Neues Optik J 1980;15:569-71.

78. Klyce S, Wilson S. Methods of Analysis of Corneal Topography. Refract Corneal Surg 1989;5:368-371.

79. Wilson SE, Klyce SD, Husseini ZM. Standardized color-coded maps for corneal topography. Ophthalmology 1993;100:1723-7.

80. Maeda N, Klyce SD, Smolek MK, Hamano H, Mitsunaga S, Watanabe K. Videokeratography for quantitative surface analysis of used soft contact lenses. Jpn J Ophthalmol 1997;41:235-9.

81. Jongsma FH, de Brabander J, Hendrikse F, Stultiens BA. Development of a wide field height eye topographer: validation on models of the anterior eye surface. Optom Vis Sci 1998;75:69-77.

82. Jongsma FHM, Brabander de J, Hendrikse F. Review and Classification of Corneal Topographers. Lasers Med Sci 1999;14:2-19.

83. Hirst E. Fitting a true asperical contact lens. Contacto 1974;1:15-9.

84. Kok JH, Smulders F, van Mil C. Fitting of aspheric high gas-permeable rigid contact lenses to scarred corneas. Am J Ophthalmol 1991;112:191-4.

85. La Hood D. Edge shape and comfort of rigid lenses. Am J Optom Physiol Opt 1988;65:613-8.

86. Filderman IP, White PF. Contact Lens Practice and Patient Management. Ed. 1, Philadelphia, Chilton Book Company, 1969.

87. Obrig TE, Salvatori PL. Contact Lenses. Ed. 3, New York, Obrig Laboratories Inc., 1957.

88. Worp E, Morgan P. Enquete naar de contactlensaanpassingen in Nederland en Groot-Brittanie. Visus 1999:9-13.

89. Loan N. Psychological, clinical and commercial perspectives on contact lenses. Contact Lens and Anterior Eye 2000;23:36-43.

90. World HO. WHO report 1998, life in the 21ste century. A vision for all. Geneva, WHO, 1989.

91. Farkas P. Breaking the Contact Lens Barrier. Optom Economics 1990:28-31.

92. Krajewski R. Presbyopic Lenses: An Update. Contact Lens Forum 1989:40-1.

93. Vegt van der GJ. Kwantitatief marktonderzoek aangaande contactlenzen. Emmen, Hogeschool Drenthe, 1999.

94. 1) Morgan P, Efron N. Trends in UK contact lens prescribing 2000. Optician 2000;219(5749):22-3.

2) Edwards K, Hough T. Fear and Loathing in Surfer's Paradise. On the demise of rigid contact lenses - a critique. Global Contact 2002;30:25-31.

95. Kohlhammer K, Zirkmann K. Der Kontaktlinsenmarkt zeigt sich sprunghaft. Kontaktlinse 2000:3233.

96. Kok JHC. New developments in the field of contact lenses. Thesis, Amsterdam, University of Amsterdam, 1991. ISBN 9060763394.

97. Mandell RB, Polse KA. Contact lenses worn during sleep and rest periods. J Am Optom Assoc 1970;41:937-9.

98. Roscoe WR, Hill RM. Corneal oxygen demands: a comparison of the open- and closed-eye environments. Am J Optom Physiol Opt 1980;57:67-9.

99. Holden BA, Sweeney DF, Sanderson G. The minimum precorneal oxygen tension to avoid corneal edema. Invest Ophthalmol Vis Sci 1984;25:476-80.

100. Holden BA, Mertz GW. Critical oxygen levels to avoid corneal edema for daily and extended wear contact lenses. Invest Ophthalmol Vis Sci 1984;25:1161-7.

101. Holden BA, Tan KO, Sack RA. The closed-eye challenge. Adv Exp Med Biol 1994;350:427-30. 
102. Liesegang TJ. Contact lens-related microbial keratitis: Part II: Pathophysiology. Cornea 1997;16:26573.

103. Liesegang TJ. Contact lens-related microbial keratitis: Part I: Epidemiology. Cornea 1997;16:125-31.

104. Holden BA, Swarbrick HA. Extended wear lenses. Contact Lenses: A Textbook for Practitioner and Student. Ed. 3; Chapter 15:555-62. Edited by Phillips AJ, Stone J. London, Butterworths, 1989.

105. Aquavella JV. New aspects of contact lenses in ophthalmology. Adv Ophthalmol 1976;32:2-34.

106. Goodlaw E. Contact lens solutions. J Am Optom Assoc 1976;47:367.

107. Ruben M. Review: contact lens in practice. Ann Ophthalmol 1981;13:289-95.

108. Hill RM. The Glenn A. Fry Award lecture: eye, lens, and care system: a menage a trois? Am J Optom Physiol Opt 1984;61:431-6.

109. Fatt I. Prentice Medal lecture: contact lens wettability—myths, mysteries, and realities. Am J Optom Physiol Opt 1984;61:419-30.

110. A discussion paper on contact lens maintenance systems. International Committee on Contact Lenses. Ann Ophthalmol 1992;24:352-7.

111. Engle JP. Caring for contact lenses. Am Pharm 1994;3:73-82.

112. Bowden FWd, Cohen EJ, Arentsen JJ, Laibson PR. Patterns of lens care practices and lens product contamination in contact lens associated microbial keratitis. CLAO J 1989;15:49-54.

113. Sokol JL, Mier MG, Bloom S, Asbell PA. A study of patient compliance in a contact lens-wearing population. CLAO J 1990;16:209-13.

114. Turner FD, Gower LA, Stein JM, Sager DP, Amin D. Compliance and contact lens care: a new assessment method. Optom Vis Sci 1993;70:998-1004.

115. Claydon BE, Efron N. Non-compliance in contact lens wear. Ophthalmic Physiol Opt 1994;14:35664.

116. Sibley M. Current contact lens care systems. Int Ophthalmol Clin 1991;31:13-6.

117. Pedersen NB. Allergy to chemical solutions for soft contact lenses [letter]. Lancet 1976;2:1363.

118. Morgan JF. Complications associated with contact lens solutions. Ophthalmology 1979;86:1107-19.

119. Josephson JE, Caffery BE. Hydrogel lens solutions. Int Ophthalmol Clin 1981;21:163-71.

120. Mondino BJ, Salamon SM, Zaidman GW. Allergic and toxic reactions of soft contact lens wearers. Surv Ophthalmol 1982;26:337-44.

121. Miller JR. Sensitivity to contact lens solutions [letter]. West J Med 1984;140:791.

122. Fisher AA. Allergic reactions to contact lens solutions. Cutis 1985;36:209-11.

123. Mackie IA. Medical contact lens practice: a systematic approach. Ed. 1 London, ButterworthHeinemann., 1993.

124. Lowther GE, Hilbert JA. Deposits on hydrophilic lenses: differential appearance and clinical causes. Am J Optom Physiol Opt 1975;52:687-92.

125. Bilbaut T, Gachon AM, Dastugue B. Deposits on soft contact lenses. Electrophoresis and scanning electron microscopic examinations. Exp Eye Res 1986;43:153-65.

126. Allansmith MR, Korb DR, Greiner JV, Henriquez AS, Simon MA, Finnemore VM. Giant papillary conjunctivitis in contact lens wearers. Am J Ophthalmol 1977;83:697-708.

127. Fowler SA, Allansmith MR. Evolution of soft contact lens coatings. Arch Ophthalmol 1980;98:95-9.

128. Collins MJ, Carney LG. Patient compliance and its influence on contact lens wearing problems. Am J Optom Physiol Opt 1986;63:952-6.

129. Farkas P, Kassalow TW, Farkas B. Contact lens practice compliance. J Am Optom Assoc 1987;58:228-9.

130. Chun MW, Weissman BA. Compliance in contact lens care. Am J Optom Physiol Opt 1987;64:274-6. 
131. Trick LR. Patient compliance-don't count on it! J Am Optom Assoc 1993;64:264-70.

132. Fatt I. Oxygen Transmission. Contact Lenses the CLAO Guide to Basic Science and Clinical Practise. Chapter 7:113-203. Edited by Kastl P., Kendall/Hunt, 1995.

133. Hofer P. How important are standards for our industry? Global Contact 2000;22:31-34.

134. Hough T. A guide to Contact Lens Standards. London, BCLA, 2000.

135. Lembach RG, Keates RH. Aspheric silicone lenses for keratoconus. CLAO J 1984;10:323-5.

136. Roth HW, Iwasaki W, Takayama M, Wada C. Complications caused by silicon elastomer lenses in West Germany and Japan. Contacto 1980;24:28-36.

137. Rogers GL. Extended wear silicone contact lenses in children with cataracts. Ophthalmology 1980;87:867-70.

138. Matsumoto ER, Murphree AL. The use of silicon elastomer lenses in aphakic pediatric patients. Internat eye care 1986;2:214-7.

139. Bier N. A study of the cornea in relation to contact lens practice. Am J Optom Physiol Opt 1956;33:291-304.

140. Dickinson F. Report on a new corneal lens. Optician 1954;128:3-6.

141. Bier N. The Contour Lens. Contact Lens Routine and Practice. Ed. 2;p193-206. London, Butterworths, 1957.

142. Filderman IP, White PF. Recent Developments. Contact Lens Practice and Patient Management. Ed. 1;p 390-400. Philadelphia, Chilton Book Company, 1969.

143. Guillon M, Lydon DP, Wilson C. Corneal topography: a clinical model. Ophthalmic Physiol Opt 1986;6:47-56.

144. Mandell RB. The Enigma of the Corneal Contour. CLAO J 1992;18:267-73.

145. Mandell RB, Klein SA. The cornea is not an ellipse. Optom Vis Sci 1998;75;12s:156.

146. Artal P, Brabander de J, Chateau N, Marin G. Contact Lenses with Aspherical and Asymmetric Surfaces (CLAAS). Brussels, Brite-Euram, 1998. Also see www.claasproject.net.

147. Castellano CF, Myers RI, Becherer PD, Walter DE. Rotational characteristics and stability of soft toric lenses. J Am Optom Assoc 1990;61:167-70.

148. McMonnies CW. After-care symptoms, signs and management. Contact Lenses: A Textbook for Practitioner and Student. Ed. 3; Chapter 19:701-36. Edited by Phillpips AJ, Stone J. London, Butterworths, 1989.

149. Phillips AJ, Stone J. Contact Lenses: A Textbook for Practitioner and Student, 3 ed. London, Butterworth, 1989.

150. Brabander de J. Het endotheel sleutel tot cornea fysiologie. Utrecht, ANVC, 1980.

151. Mandell RB, Kang L. Rigid contact lens area and corneal oxygenation. Am J Optom Physiol Opt 1988;65:387-94.

152. Sarver MD, Harris MG. Corneal lenses and "spectacle blur". Am J Optom Arch Am Acad Optom 1967;44:502-4.

153. Brungardt TF, Potter CE. Spectacle blur refraction of long time contact lens wearers. Am J Optom Arch Am Acad Optom 1971;48:418-25.

154. Friedberg MA. Spectacle blur. J Am Optom Assoc 1976;47:355.

155. Sampson WG, Soper JW, Girard LJ. Topographical keratometry and contact lenses. Trans Am Acad Ophthalmol Otolaryngol 1965;69:959-69.

156. Rengstorff RH. Corneal curvature and astigmatic changes subsequent to contact lens wear. J Am Optom Assoc 1965;36:996-1000. 
157. Bailey IL, Carney LG. Analyzing contact lens induced changes of the corneal curvature. Am J Optom Arch Am Acad Optom 1970;47:761-8.

158. Levenson DS, Berry CV. Findings on follow-up of corneal warpage patients. CLAO J 1983;9:126-9.

159. Briceno Garbi EA. Variations in corneal curvature and refractive error in CAB gas-permeable contact lens wearers. J Am Optom Assoc 1984;55:217-9.

160. Sarver MD, Nelson JL, Polse KA. Peripheral corneal staining accompanying contact lens wear. J Am Optom Assoc 1969;40:310-3.

161. Korb DR, Korb JM. A study of three and nine o'clock staining after unilateral lens removal. J Am Optom Assoc 1970;41:233-6.

162. Wechsler S. Corneal insult and staining—hard lenses. J Am Optom Assoc 1976;47:353.

163. Mandell RB. Contact Lens Practice. Ed. 2, Springfield, Charles C Thomas, 1977.

164. Norn MS. Rose bengal vital staining. Staining of cornea and conjunctiva by 10 prcent rose bengal, compared with 1 percent. Acta Ophthalmol Copenh 1970;48:546-59.

165. Kline LN, DeLuca TJ, Fishberg GM. Corneal staining relating to contact lens wear. J Am Optom Assoc 1979;50:353-7.

166. Guillon JP, Guillon M, Malgouyres S. Corneal desiccation staining with hydrogel lenses: tear film and contact lens factors. Ophthalmic Physiol Opt 1990;10:343-50.

167. Begley CG, Barr JT, Edrington TB, Long WD, McKenney CD, Chalmers RL. Characteristics of corneal staining in hydrogel contact lens wearers. Optom Vis Sci 1996;73:193-200.

168. Kline LN, Deluca TJ. Arcuate staining. J Am Optom Assoc 1976;47:360.

169. Grosvenor T. Visual acuity, astigmatism and soft contact lenses. Am J Optom Arch Am Acad Optom 1972;49:407-12.

170. Wechsler S. Visual acuity in hard and soft contact lens wearers: a comparison. J Am Optom Assoc 1978;49:251-6.

171. Wilson MS. Corneal oedema from corneal contact lens wear, its causes and treatment. Trans Ophthalmol Soc U K 1970;90:31-45.

172. Readshaw G. Assessment of corneal oedema in contact lens wearers. Aust J Ophthalmol 1973;1:27-9.

173. Polse KA, Mandell RB. Etiology of corneal striae accompanying hydrogel lens wear. Invest Ophthalmol 1976;15:553-6.

174. Health Council of the Netherlands: Committee on Health risks of contact lenses. Health risks of contact lenses. The Hague: Health Council of the Netherlands, 2001; publication no. 2001/20.

175. Terry RL, Schnider CM, Holden BA, Cornish R, Grant T, Sweeney D, La Hood D, Back A. CCLRU standards for success of daily and extended wear contact lenses. Optom Vis Sci 1993;70:234-43.

176. Mandell R. Morphometry of the Human Cornea, thesis, Indiana University, 1962.

177. Mandell RB. Methods to measure the peripheral corneal curvature. J Am Optom Ass 1962:889-92.

178. Placido A. Novo instrumento de esploracao da cornea. Per d'Oftalmol Pract 1880;5:27-30.

179. Gullstrand A. Photographisch-ophthalmometrische und klinische Untersuchungen uber die Hornhautrefraktion. 1896:28. 
Chapter 2

Contact Lenses in Infant Aphakia and as Artificial Iris 


\subsection{Introduction to this chapter}

The high oxygen transmissibility properties of CLs solely based on silicone is advantageous in cases that demand extreme centre or edge thickness of the lens, e.g. in high plus power (aphakia) or high minus power (myopia gravior). Research on silicone as a material for CLs started rather early. ${ }^{1-4}$ However, a major difficulty to overcome is the hydrophobic nature of silicone. 5,6 There have been some controversies in the literature on the application of silicone $\mathrm{CLs}^{7-15}$, mainly concerning the extreme formation of surface deposits in adults. However, the use of silicone CLs for correction of aphakia in children appears to be acceptably feasible. ${ }^{16-19}$ Since permanent wear is important in correcting infant aphakia we studied the application of silicone CLs in young children over a relatively long time period. The report on this research can be found in section 2.2 of this thesis.

The topic of study in section 2.3 are SCLs as prosthesis. The large diameter and good centring properties make SCLs ideal in cases where an artificial iris or an occluding pupil is needed. However, low water content, very thick, so-called 'sandwich lenses' 20,21 with 'hand painted' irises are difficult to fit, can cause physiological problems $\mathrm{s}^{22}$, are very expensive and difficult to reproduce. We studied the success rate of a system using relatively inexpensive, standardised thin high water content SCLs in different indications for artificial iris and/or black occluding pupil.

\section{References}

1. Hill RM. Effects of a silicone rubber contact lens on corneal respiration. J Am Optom Assoc 1966;37:1119-21.

2. Hill RM, Schoessler J. Optical membranes of silicone rubber. J Am Optom Assoc 1967;38:480-3.

3. Burns RP, Roberts H, Rich LF. Effect of silicone contact lenses on corneal epithelial metabolism. Am J Ophthalmol 1971;71:486-9.

4. Zekman TN, Sarnat LA. Clinical evaluation of the silicone corneal contact lens. Am J Ophthalmol 1972;74:534-7.

5. Koziol JE, Peyman GA, Yasuda H. Evaluation of a new silicone-methane polymer contact lens. Arch Ophthalmol 1983;101:1779-81.

6. Rosner M, Treister G, Blumenthal M. Corneal abscesses in silicone and soft contact lens wearers. Ann Ophthalmol 1983;15:949-52.

7. Dahl AA, Brocks ER. The use of continuous-wear silicone contact lenses in the optical correction of aphakia. Am J Ophthalmol 1978;85:454-61.

8. Ruben M, Guillon M. 'Silicone rubber' lenses in aphakia. Br J Ophthalmol 1979;63:471-4.

9. Aquavella JV, Rao GN. Which lens: contact lenses currently available for extended wear in aphakia. Ophthalmology 1980;87:151-4.

10. Hill JF, Anderson FL, Johnson TK, Rigel LE, Seelye RR. Eighteen-month clinical experience with extended wear silicone contact lenses on 400 patients. Am J Optom Physiol Opt 1983;60:578-81.

11. Mizutani Y, Goto J, Murakami M, Mizutani Y. Clinical experience in fitting 370 patients with the silicone elastomer contact lens, Hisilic II. CLAO J 1983;9:249-53. 
12. Blackhurst RT. Silicone contact lenses: a personal 13-year perspective. CLAO J 1985;11:57-61.

13. Sweeney DF, Holden BA. Silicone elastomer lens wear induces less overnight corneal edema than sleep without lens wear. Curr Eye Res 1987;6:1391-4.

14. Holden BA, Sweeney DF. Complications with silicone lens wear [letter]. Am J Optom Physiol Opt $1988 ; 65: 765$.

15. Kossovsky N. Can the silicone controversy be resolved with rational certainty? [editorial]. J Biomater Sci Polym Ed 1995;7:97-100.

16. Gurland JE. Use of silicone lenses in infants and children. Ophthalmology 1979;86:1599-1604.

17. Rogers GL. Extended wear silicone contact lenses in children with cataracts. Ophthalmology 1980;87:867-70.

18. Cutler SI, Nelson LB, Calhoun JH. Extended wear contact lenses in pediatric aphakia. J Pediatr Ophthalmol Strabismus 1985;22:86-91.

19. Harris M. Correction of pediatric aphakia with silicone contact lenses. CLAO J 1985;11:343-7.

20. Bier N. Prosthetic correction. Am J Optom Physiol Opt 1982;59:178-83.

21. Port, M. Cosmetic and prosthetic contact lenses. Contact Lenses, chapter 21:p789-95, edited by Phillips AJ and Stone J. London, Butterworths, 1989.

22. Dickinson GM, Bisno AL. Infections associated with prosthetic devices: clinical considerations. Int J Artif Organs 1993;16:749-54. 


\subsection{Silicone Contact Lenses in Infant Aphakia}

A Practical Approach to and Long-Term Results of Fitting Silicone Contact Lenses in Aphakic Children after Congenital Cataract

J. de Brabander ${ }^{1,3}$, J.H.C. Kok ${ }^{2,3}$, R.M.M.A. Nuijts' ${ }^{1}$ and L.J.J.M. Wenniger-Prick3.

From:

1 Eye Research Institute Maastricht, Department of Ophthalmology Academic Hospital, University Maastricht, the Netherlands.

2 Medical Center Jan van Goyen, Amsterdam, the Netherlands.

3 Department of Ophthalmology, Oculenti practice, Academic Medical Center at the University of Amsterdam, the Netherlands.

The CLAO Journal 2002;28;1:31-5. 
Abstract

Purpose. This prospective study investigated characteristics of fit and performance of silicone contact lenses under conditions of permanent wear in cases of aphakia after congenital cataract.

Methods. Seventeen aphakic children (8 eyes unilateral, 18 eyes bilateral) were fitted with silicone contact lenses on an empirical basis without the use of keratometry. The lenses were worn on a permanent basis, with a routine replacement every 3 months. The follow-up was 6 years, with evaluation of lens parameters, fitting characteristics, lens condition, replacement rate, wearing time, complications, and visual acuity.

Results. The back optical zone radius (BOZR) remained stable at $7.50 \mathrm{~mm}$, up to the age of 1.5 years old. At the age of 4 years, almost all eyes needed a BOZR of $7.90 \mathrm{~mm}$. Mean \pm SD contact lens power was +25.47 diopter $(\mathrm{D}) \pm 4.0($ range $+32.00 \mathrm{D}$ to $+20.00 \mathrm{D})$ at 3 months of age, and $+17.94 \mathrm{D} \pm 3.8$ (range $+29.00 \mathrm{D}$ to $+15.00 \mathrm{D}$ ) at 3 years of age. This represents an average decrease of $0.23 \mathrm{D}$ per month. The power decrease in unilateral cases was significantly lower $(\mathrm{p}<0.01)$ than in bilateral aphakia. Snellen visual acuity at 4 years of age was 0.1 to $0.3(20 / 200-20 / 60)$ in 15 eyes, 0.3 to $0.5(20 / 60-20 / 40)$ in 10 eyes and greater than $0.5(>20 / 40)$ in 1 eye. Lens usage over a total period of 90 wearing years was 5.6 lenses/eye/year, including the regular exchange every 3 months. No serious complications occurred. A positive correlation $(r=0.89)$ was found between age and deposit build up. With the need for permanent wear reduced, therefore, $73 \%$ of the eyes were refitted with high-water content soft lenses or high gas permeable (HGP) rigid lenses, when the patients ranged in age from 4 to 6 years old.

Conclusion. The procedure for fitting of silicone lenses in aphakic children is feasible in an easy and logical way without keratometry. Permanent wear with low complication rate is possible up to the age of 3 , with a lens usage (including a 3-month regular exchange) of 5.6 lenses/eye/year. From the age of 3 , deposit formation is the determining factor for refitting with high-water content soft or HGP rigid lenses.

Key words:

congenital cataract, infant aphakia, contact lenses, permanent wear, silicone elastomer. 


\subsubsection{Introduction}

Congenital cataract is one of the leading causes of form deprivation amblyopia in children. ${ }^{1,2}$ Surgical correction is essential at an early age to prevent amblyopia, strabismus, or poor fusion and should be followed by immediate and permanent optical correction of the resulting aphakia. Preferably, treatment should start within the critical period of the visual system, 17 weeks after birth. ${ }^{3-6}$

Spectacles have the disadvantage of distorted peripheral images, constriction of the visual field, and problems of low compliance. Intraocular lenses and epikeratophakia are difficult to use because of the subsequent growth of the infant eye, resulting in change of necessary refractive power. Furthermore, the long-term effects of intraocular lenses in infants eyes are not known. Early contact lens fitting seems to be the most practical solution for correction of infant aphakia. 7,8

Fitting contact lenses in infant aphakia involves several characteristic problems. Measuring the eye parameters of such young children is not easy, and assessment of the fit on the eye with a routine slit lamp examination is practically impossible. Usually, keratometry and evaluation of the lens fit need to be done under sedation or general anesthesia, in the operating room. ${ }^{9}$ The necessary, high plus refractive power leads to lenses with remarkable center thickness. To avoid decentration (especially low riding) or lens loss, the lens diameter must often be taken as large as the cornea, and a relative steep fit is indicated. The insertion and removal of the lenses on a daily-wear basis may be a stressful event for both parents and child. Because the young aphakic eye must be corrected during all sporadic waking hours ${ }^{10}$, extended wear is a key demand to the successful treatment, at least up to an age of 3 to 4 years old.

For these reasons, the use of rigid contact lenses has not been successful. The high risk of lens loss and the chance of lens binding on the cornea are further complications. High-water content soft contact lenses, although comfortable to wear and easy to fit, show a high rate of complications due mainly to reduced oxygen transmission in the high-plus thick lens modality. 9 Therefore, contact lenses made of silicone elastomer with high oxygen permeability have the potential to meet the high demands in the correction of young aphakic children.

Silicone contact lenses have had a long and troublesome history of development. ${ }^{11-14}$ Since the experiments of Becker, in 1956, the material, lens design, production, and reproducibility improved, but only slowly. In September 1984 the Silsoft (Bausch \& Lomb, Rochester, NY) lens, manufactured from a silicone elastomer, was the first lens that reached U.S. Food and Drug Administration (FDA) approval as a 30-day extended-wear lens for aphakia. ${ }^{13}$ The basic material is hydrophobic, and, therefore, a surface treatment (with the formation of hydroxyl groups on the lens surface) is required. This process gives the lens a low contact angle without making it hydrophilic. ${ }^{15}$ This means that there is no dehydration in low humidity, and the more stable lens usually gives a better visual acuity than hydroxyethyl methacrylate (HEMA) lenses. ${ }^{16}$ The material is soft but stable enough to assure good optical quality and high resistance to tearing; and it has a good thermal conductivity. 15-17 The most important feature of silicone elastomer is its high oxygen permeability. Equivalent 
Oxygen Percentage (EOP) is a measure for the amount of oxygen that is available to the cornea. The baseline of the EOP scale is $21 \%$ oxygen available from air, without contact lens wear. For safe, permanent wear (including closed-eye conditions), a minimal EOP value of $18 \%$ has been indicated. Hill ${ }^{18}$ showed that Silsoft lenses have an average EOP of $18.7 \%$ and $18.4 \%$ in lenses that had been worn more than 12 hours per day during an 8 to 10 months period. Barr ${ }^{15}$ demonstated a graph from the work of Fizgerald yields approximately the same EOP value but also shows that the EOP value is independent of lens thickness. Oxygen transmissibility of a contact lens can also be given as the $\mathrm{Dk} / \mathrm{L}$ value. The oxygen permeability of the material is Dk, and $\mathrm{L}$ is the lens thickness. The Dk value of Silsoft lenses is stated by the manufacturer as $340 \times 10^{-11} \mathrm{~cm}^{2} \mathrm{~mL} \mathrm{O}_{2} / \mathrm{sec} \mathrm{mL} \mathrm{mmHg}$. Glasser and Weissman ${ }^{19}$ found a value of only 50, whereas Weissman, Fatt and Pham ${ }^{20}$ reported a value of 190 using a single-chamber polarographic technique. The high standard deviation $(\mathrm{SD}=79)$ found in the latter study led the authors to suggest that better methods should be developed to evaluate oxygen performance of high-Dk materials. According to the same study, lens thickness does correlate with oxygen transmissibility, but, even with a very thick lens, oxygen transmissibility is high enough to meet the demand for permanent wear $(\mathrm{Dk} / \mathrm{L}=90)$.

The aim of this long-term prospective study was to investigate the performance of Silsoft contact lenses under permanent wear conditions in children with aphakia after congenital cataract.

\subsubsection{Subjects and Methods}

An important patient-intake criterion in this study was an early diagnosis to ensure that the cataract was congenital. ${ }^{21}$ All patients in this study underwent surgery at the Academic Medical Center, University of Amsterdam, the Netherlands. Normal standards were followed to established a multidisciplinary diagnosis, prognosis, pre- and postoperative care, and informed consent.

All aphakic eyes were fitted with Silsoft contact lenses (Table 1) as soon as possible after surgery. The fitting of the lenses was performed without sedation in the consulting room, and the fitting procedure did not involve keratometry. In all cases, a lens with a BOZR of $7.50 \mathrm{~mm}$ and a total diameter (TD) of $11.3 \mathrm{~mm}$ was inserted as first choice. Using a pen-

\begin{tabular}{l} 
Table 1. Properties of Silsoft contact lenses. \\
Silicone elastomer \\
Surface treatment \\
Soft, stable optics \\
Dk 340, independent of thickness \\
BOZR 7.50; $7.70 ; 7.90 \mathrm{~mm}$ \\
TD 11.3; $12.5 \mathrm{~mm}$ \\
\hline
\end{tabular}


light, a magnifying lens (+10 D), and an ophthalmoscope or hand-held slitlamp, the motility and centering of the lens were evaluated 30 minutes after insertion. If the lens moved too much or decentered, the TD was increased to $12.5 \mathrm{~mm}$. In cases of insuffcient motility, a lens with a flatter BOZR was used and evaluated again. Lens power was calculated from retinoscopy. An addition of $+4 \mathrm{D}$ (counted from birth) was given to assure optimal focussing at short distance. This addition was decreased by $1 \mathrm{D}$ each year. Parents were directed to routinely check the eyes of their child. They were instructed to look for a clear reflex of the front surface because this indicates the wettability of the lens. If dull areas were present, parents were to apply a saline solution containing no preservative. Parents were also directed to routinely insert one drop of saline (no preservatives) when the child awakened. Parents were taught how to remove, clean, and insert the lens and were advised that cleaning should only be done in case re-wetting failed. As long as the surface was wet and the eye remained clear, the lenses were worn permanently. If the eyes became red or there was any doubt about the lens or eye condition, the parents were instructed to consult the clinic immediately.

Follow-up visits were scheduled at 1 night, 1 week, 1 month, and every 3 months thereafter. During follow-up visits, an ophthalmologic examination was performed that included assessment of movement, centration, and condition of the lens. The refractive power of the lens was checked using retinoscopy with and without the lens in situ. Lenses were routinely exchanged every 3 months. To make sure no wearing time was lost due to delay in ordering of lenses, spare lenses were stocked in the clinic for all children.

\subsubsection{Results}

Seventeen childeren completed this study. Eight ( 2 male, 6 female) patients suffered from unilateral congenital cataract, and nine ( 4 male, 5 female) patients suffered from bilateral congenital cataract (Table 2). A total of 26 eyes were treated. The etiology of the congenital cataract was hereditary (3), associated with Down syndrome (3), Rubella infection and microphthalmos (2), and, in one case, associated with encephalopathy. Typically, in the remaining 8 cases (47\%), the etiology was idiopathic. In 3 eyes, persistent hyperplastic primary vitreous (PHPV) was present. Surgical technique consisted of lensectomy with anterior vitrectomy in all eyes.

\begin{tabular}{|c|c|c|c|}
\hline Gender & Unilateral & Bilateral & Total \\
\hline Male & 2 & 4 & 6 \\
\hline Female & 6 & 5 & 11 \\
\hline Total patients & 8 & 9 & 17 \\
\hline Total eyes & 8 & 18 & 26 \\
\hline
\end{tabular}


The time period between birth and the start of contact lens wear averaged 20.3 weeks and ranged from 8.6 to 41 weeks. This wide spread in time period could already be found in the time interval between birth and diagnosis, averaging 11.2 weeks and ranging from 1 to 22 weeks. Surgery was performed at an average of 6.7 weeks after the diagnosis (range 1-26 weeks). Contact lens wear was started at an average of 2.4 weeks (range 1 day-9.5 weeks) after surgery. Snellen visual acuity at the age of 4 years is listed per eye and per type of aphakia in Table 3. The typical discrepancy between visual acuity in bilateral and unilateral cases can be seen. Visual acuity in bilateral cases was significantly better (chi squared $\mathrm{p}<$ 0.05 ) than that of the treated eye in unilateral aphakia.

\begin{tabular}{|c|c|c|c|}
\hline Snellen visual acuity & Unilateral & Bilateral & Total \\
\hline$<0.1(<20 / 200)$ & 3 & 2 & 5 \\
\hline $0.1-0.3(20 / 200-20 / 60)$ & 4 & 6 & 10 \\
\hline $0.3-0.5(20 / 60-20 / 40)$ & 1 & 9 & 10 \\
\hline $0.5-0.7(20 / 40-20 / 30)$ & 0 & 1 & 1 \\
\hline$>0.7(>20 / 30)$ & 0 & 0 & 0 \\
\hline Total eyes & 8 & 18 & 26 \\
\hline
\end{tabular}

Few complications occured until patients reached the age of 3 years (cumulative wearing time 72 years). In 4 eyes ( 2 patients), a bacterial conjunctivitis caused by Haemophilus influenzae occurred. Both cases resolved without sequelae. In one case an acute red eye was associated with lens binding, and a flatter lens solved the problem. In 4 eyes, decentration of the lens was better corrected using a $12.5-\mathrm{mm}$ lens compared to a $11.3-\mathrm{mm}$ lens with steeper base curve.

Lens power at 3 months of age showed a large range (+ 26 to $+32 \mathrm{D})$ that was equal for both unilateral and bilateral aphakia. In Figure 1, the distribution of mean \pm SD of lens power, from 3 to 36 months old, is given for bilateral and unilateral aphakia. The mean \pm SD contact lens power at 3 months of age for the total group $(+28.40 \mathrm{D} \pm 2.76)$, the bilateral group $(+28.00 \mathrm{D} \pm 3.10)$, and the unilateral group $(+29.00 \mathrm{D} \pm 2.45)$ were not statistically different $(\mathrm{p}>0.01)$. With increasing age, the power spread in bilateral cases generally channels into a lower range of +15 to $+18 \mathrm{D}$ (mean $+16.44, \pm 1.24)$ at 3 years of age. This is not so much the case in the group of unilateral aphakia, with a range of +16 to $+29 \mathrm{D}$ (mean $+19.63, \pm 5.01)$. The effect on the SD is entirely due to the two cases of unilateral aphakia with the etiology of Rubella associated microphthalmos. Excluding these two cases from the analysis in the unilateral group reveals a power range of +26 to $+29 \mathrm{D}$ (mean $27.50 \mathrm{D}, \pm$ $2.68)$ at 3 months of age and +16 to $+18 \mathrm{D}($ mean $+17 \mathrm{D}, \pm 1.1)$ at 3 years of age. The latter figures are not statistically diffent $(\mathrm{p}>0.1)$ from the bilateral group.

The average change in power per month of age for the total group, the bilateral group, and the unilateral group were $0.32 \mathrm{D}, 0.35 \mathrm{D}$, and $0.28 \mathrm{D}$, respectively. If the two cases of Rubella associated with microphthalmos are exclueded from the unilateral group, the change 


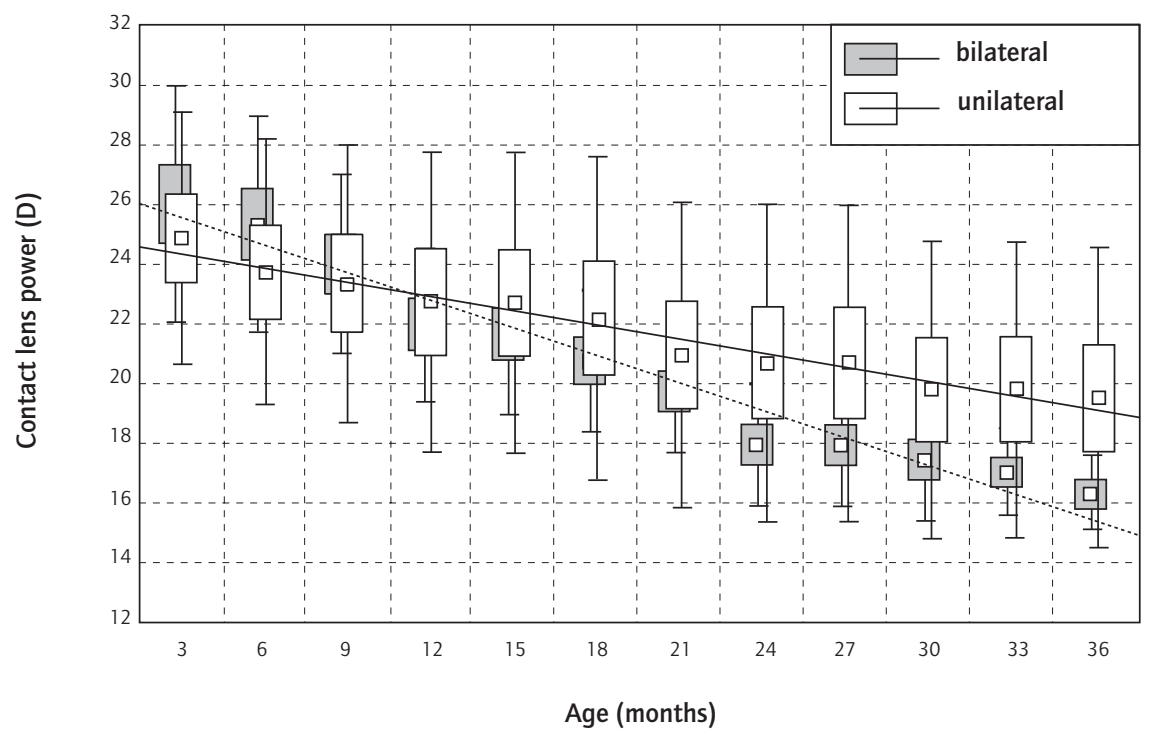

Figure 1. Change of contact lens power with age in bilateral and unilateral aphakia.

in power per month of age is $0.31 \mathrm{D}$. The individual cases of Rubella showed power changes of $0.18 \mathrm{D}$ and $0 \mathrm{D}$ per month of age. BOZR changes follow a remarkable line (Table 4). Until the age of 1.5 years, almost all eyes needed a BOZR of $7.50 \mathrm{~mm}$. Within the next 2 years, significant changes are made to a BOZR of 7.70 and $7.90 \mathrm{~mm}$. At around the age of 3 years, deposit formation increased rapidly and patients were refitted with hydrophilic or HGP rigid lenses under daily wear conditions (see Table 4). A further analysis of the age relation for the total of 60 extra replacements for deposits revealed a significant positive correlation with age $(\mathrm{r}=0.89$ at $\mathrm{p}<0.05)$.

\begin{tabular}{|c|c|c|c|c|c|c|c|c|}
\hline Type of lens & age & 0.5 & 1 & 2 & 3 & 4 & 5 & 6 \\
\hline Silsoft BOZR $7.50 \mathrm{~mm}$ & & 19 & 24 & 11 & 3 & 1 & 1 & 1 \\
\hline Silsoft BOZR $7.70 \mathrm{~mm}$ & & 1 & 2 & 9 & 6 & 2 & 1 & 1 \\
\hline Silsoft BOZR $7.90 \mathrm{~mm}$ & & 0 & 0 & 6 & 17 & 10 & 8 & 5 \\
\hline Hydrophilic & & 0 & 0 & 0 & 0 & 13 & 14 & 15 \\
\hline High Gas Permeable & & 0 & 0 & 0 & 0 & 0 & 2 & 4 \\
\hline Total eyes & & 20 & 26 & 26 & 26 & 26 & 26 & 26 \\
\hline
\end{tabular}

Table 5 gives the number of lenses needed up to the age of 4 years (cumulative wearing time 90 years) and the reasons for lens replacements. Although the standard procedure included 
replacement of lenses every 3 months, this turned out to be lower than planned (3.2 lenses/eye/year) because routine replacements were combined with lenses replaced for other, specific reasons. Extra lens replacements per eye per year were made, in order of importance, because of deposits (0.7), power (0.7), BOZR (0.4), lens loss (0.4), defects (0.2) and diameter (0.04). The total average number of lenses used per eye/year was 5.6. A dramatic spread in lens usage per child was found. Some children needed only the routine 4 lenses per eye/year, whereas others used many more lenses; results showed that replacement also was often needed for the same reason per child.

\begin{tabular}{|c|c|c|}
\hline Reason for change & Absolute & Per eye / year \\
\hline Defect & 20 & 0.2 \\
\hline Loss & 34 & 0.4 \\
\hline Deposits & 60 & 0.7 \\
\hline Power & 64 & 0.7 \\
\hline BOZR & 34 & 0.4 \\
\hline Diameter & 4 & 0.04 \\
\hline Routine & 288 & 3.2 \\
\hline Total & 504 & 5.6 \\
\hline
\end{tabular}

\section{Discussion}

Generally, the results on the fitting procedure with Silsoft contact lenses in aphakia after congenital cataract are in accordance with data found by Lightman and Marshall.22 It was also found that, up to 6 months of age, a BOZR of $7.50 \mathrm{~mm}$ and a diameter of $11.3 \mathrm{~mm}$ are good starting points for succesful fitting without keratometry. In some cases, increasing the diameter was a better method for preventing lens decentration than fitting with a steeper BOZR. In our study, the crucial period in which patients needed changes in BOZR also started around the age of 18 months. The shift in BOZR to 7.70 and $7.90 \mathrm{~mm}$ takes place between 1.5 and 4 years of age. At age 4 years, $77 \%$ of the eyes were wearing a base curve $7.90 \mathrm{~mm}$.

Comparing the average powers needed at 6,12, 24, and 36 months to those of Lightman and Marshall,22 our study used, on average, lenses of lower power (1.8 D); however, the rate of power change in our study (0.32 D per month) is practically the same as they reported (0.35 D per month).

The two cases of microphthalmia showed a different pattern in both BOZR and power change, but there were also differences between the cases. In one case, both BOZR and power remained constant up to the age of 6 years, which would indicate no dimensional development of the eye. The other case showed a change of BOZR from $7.50 \mathrm{~mm}$ to 7.70 $\mathrm{mm}$ at an age of 2 years and a change in power from +32.00 to +26.00 from 3 to 36 months of age, indicating a change in the usual direction but less than normal. 
Results of visual acuity data in unilateral cases turned out lower than in bilateral cases. Because there was no selection concerning visual acuity prognoses in this study, this result is not surprising. This study shows that, although successful permanent optical correction is feasible in infant aphakia, it is not the only answer to visual development; this is in accordance with conclusions by Moore. ${ }^{23}$ The question remains whether it is worth the risk of surgery and the necessary intensive treatment in case of unilateral congenital cataract if visual acuity remains low. Opinions in the literature are diverse on this issue. Some studies are negative, ${ }^{24}$ others show the possibility of stimulating visual acuity but with variable results ${ }^{25,26}$ or as possible only for a select group of patients. ${ }^{27}$ Although binocularity remains a problem, there are also reports of relatively good results. ${ }^{28-30}$ With the advancements in surgical techniques, the risk of operating on infant eyes is greatly reduced, and as shown in our study, permanent optical correction is possible. The remaining issue, particularly in unilateral cases, seems to be the development of optimal orthoptic treatment schemes ${ }^{23}$ to reduce the unequal competition between the eyes. ${ }^{31}$

Some studies report difficulties in removing deposit formation, ${ }^{28,32}$ but these studies are not specific on the age of onset. Because of the higher risk of deposits associated with permanent wear, Lightman and Marshall22 recommended daily wear. In this study, deposit formation was found to become disturbing around the age of 3 years. Prior to that age, deposit formation was not a significant problem in permanent wear when combined with a routine wetting procedure. However, strict follow-ups, replacement of the lenses whenever needed, intensive instruction to the parents and parental cooperation, together with a clinical setting that provides for emergencies on a 24-hour basis are essential requirements for providing optimal care in this patient group. Also, a lens usage of 5.6 lenses per eye per year seems to be necessary to succesfully correct aphakic children after congenital cataract, with silicone contact lenses. It seems that, from the age of 4 years, silicone lenses are no longer compatible with tear composition and should, in most cases, be replaced by daily-wear high-water content soft lenses, or, at a later age, replaced with HGP rigid lenses.

\section{Acknowledgement}

With this publication we honor our dearest friend and the initiator of this study, Dr Jan H.C. Kok who died at a much too early age on November 21, 1998. We would also like to thank V.S. Gangaram Panday MD for his help in processing the data.

\section{References}

1. Lloyd IC, Goss-Sampson M, et al. Neonatal Cataract: Aetiology, Pathogenesis and Management. Eye 1992;6:184-96.

2. Sorsby A, Incidence and causes of blindness in England and Wales, 1963-68. H.M.S.O.,1972.

3. Hubel DH, Wiesel TN. Receptive fields of single neurons in the cat's striate cortex. J Physiol (Lond) 1962;148:574-91.

4. Noorden GK von. Management of congenital cataracts. Trans Acad Ophthalmol Otol 1970;74:352-59. 
5. Awaye S, Miyake S. Form deprivation amblyopia: further observations. Graefe's Arch Ophth 1988;226:132-36.

6. Cheng KP, Hiles DA, et al. Visual results after early surgical treatment of unilateral congenital cataracts. Ophthalmology 1991 Jun:98:6.

7. Dutton J, Slamovits, T,(ed.). Visual Rehabilitation of Aphakic Children. Surv of Ophthalmology 1990;34;5:365-84.

8. Hales RH. Unilateral pediatric aphakia: Current treatments and choices. Contact lens forum 1986;Nov:34-5.

9. Matsumoto ER, Murphree AL. The use of silicon elastomer lenses in aphakic pediatric patients. Internat eye care 1986;2;4:214-7.

10. Schmidt P. Clinical implications in aphakic pediatric patients. Int eye care 1986;2;4:217.

11. Baily NJ. Soft silicon lenses: the beginning. Contact lens spectrum 1987;March:17-22.

12. Fitzgerald JK. The silicon lens that works. Contact lens forum 1981;June:43-50.

13. Blackhurst RT. New flexible silicone contact lens: a thirteen month clinical experience. Contact and intraocular lens medical J 1980;6;3:252-7.

14. Baily NJ. Bausch \& Lomb pitches in for Dr. Simon. Contact lens spectrum 1987;April:53-60.

15. Barr JT. Silicon lenses are elastic and permeable. Rev of Optometry 1981;April:59-60.

16. Hill JF, Anderson FL, Johnson TK, Rigel LE, Seelye RR. Eighteen-month clinical experience with extended wear silicon contact lenses on 400 patients. Am J Optom Physiol Optics 1983;60;7:578-81.

17. Woodward EG. Therapeutic silicon rubber lenses. J British Contact Lens Ass Jan 1984;7;1:39-40.

18. Hill RM. The elastomer outlook. Contact lens forum 1980;Dec:39-41.

19. Glasser DB, Weissman BA. Mean oxygen permeability of four flexible contact lens materials. CLAO J 1983;9:29-31.

20. Weissman BA, Fatt I, Pham C. Polarographic oxygen permeability measurement of silicon elastomer contact lens material. J Am Optom Assoc 1992;63:187-90.

21. Moore BD. Pediatric cataracts—diagnosis and treatment. Optom Vis Sci 1994;71:168-73.

22. Lightman JM, Marshall D Jr. Clinical evaluation of back optic radius and power determination by age in pediatric aphakia due to congenital cataract fitted with a Silicone Elaster contact lens [published erratum appears in Optom Vis Sci 1996 May;73(5):352]. Optom Vis Sci 1996;73:22-7.

23. Moore BD. Pediatric aphakic contact lens wear: rates of successful wear. J Pediatr Ophthalmol Strabismus 1993;30:253-8.

24. Ryan SJ, Maumenee AE. Unilateral congenital cataracts and their management. Ophthalmic Surg 1977;8:35-9.

25. Enoch JM, Rabinowicz IM. Early surgery and visual correction of an infant born with unilateral eye lens opacity. Doc Ophthalmol 1976;41:371-82.

26. Cheng KP, Hiles DA, Biglan AW, Pettapiece MC. Visual results after early surgical treatment of unilateral congenital cataracts. Ophthalmology 1991;98:903-10.

27. Scott WE, Drummond GT, Keech RV, Karr DJ. Management and visual acuity results of monocular congenital cataracts and persistent hyperplastic primary vitreous. Aust N Z J Ophthalmol 1989;17:143-52.

28. Moore B. The fitting of contact lenses in aphakic infants. J Am Optom Assoc 1985;56:182-3.

29. Beller R, Hoyt CS, Marg E, Odom JV. Good visual function after neonatal surgery for congenital monocular cataracts. Am J Ophthalmol 1981;91:559-65. 
30. Pratt Johnson JA, Tillson G. Visual results after removal of congenital cataracts before the age of 1 year. Can J Ophthalmol 1981;16:19-21.

31. Birch EE, Stager D, Leffler J, Weakley D. Early Treatment of Congenital Unilateral Cataract Minimizes Unequal Competition. Invest Ophthalmol Vis Sci 1998;39:1560-66.

32. Rogers GL. Extended wear silicone contact lenses in children with cataracts. Ophthalmology 1980;87:867-70. 


\subsection{Coloured Contact Lenses as Artificial Iris}

The use of Standard thin high Water Content Artificial Iris and Black Pupil Contact Lenses

John de Brabander ${ }^{1,2}$, Cor J. van Mil², Cees J. J. Brinkman² and Rudy M.M.A. Nuijts ${ }^{1}$

From:

1 Eye Research Institute Maastricht, Department of Ophthalmology, University Maastricht, the Netherlands.

2 Medical Center at the University of Amsterdam, Department of Ophthalmology, Oculenti practice, Amsterdam, the Netherlands.

Digital Journal of Ophthalmology 2001;7:No 1. www.djo.harvard.edu. 
Abstract

Purpose. Fitting prosthetic contact lenses with artificial irises and so-called "black pupils" can be problematic due to low water content materials, thick lens designs, and the loss of color with time. In particular black pupil lenses often do not provide adequate occlusion. Furthermore, delivery times are long and it is difficult to produce the lenses such that the color of the other eye is matched to the patients satisfaction. The aim of this study was to evaluate whether the use of standardized lenses would ease prosthetic lens fitting.

Methods. In this study, 34 eyes of 31 patients were fitted with thin, high water content standardized soft contact lenses. The lenses were available in 5 standard colors with opaque iris posterior surfaces and a printed iris pattern on the anterior surface. Iris and pupil diameters are standardized to 12.5 and $5 \mathrm{~mm}$, respectively. Black occluding pupils are available in diameters from 5 to $10 \mathrm{~mm}$ in $1 \mathrm{~mm}$ steps.

Results. In $67 \%$ of eyes, the selected standard color of the lens was satisfactory to the patient. In $78 \%$ of the eyes the standard lens dimensions could be used. No adverse physiological reaction of the eyes was noticed during the one year follow-up. The iris prosthetic and black pupil lenses proved at the dispensing visit to be adequately opaque and/or totally occluding in all cases. Loss of color was, in $71 \%$ of the eyes, a reason to replace lenses between 4 to 8 months after dispensing (average 6.5 months).

Conclusion. The thin high water content standardized prosthetic lenses as used in this study provided success in $67 \%$ of the cases.

\section{Key words:}

artificial iris, black-pupil, occlusion, prosthetic soft contact lenses. 


\subsubsection{Introduction}

Hydrophilic contact lenses, with their large diameters and good centering properties, are ideal in cases where an artificial iris or occluding black pupil is indicated. There are two types of prosthetic soft lenses available. ${ }^{1,2}$ The heterogeneous type has a laminated construction whereby a printed or hand painted iris shield is sandwiched between two HEMA sections. With these lenses all iris patterns and colors can be created but their disadvantages are cost, long delivery time, poor reproducibility and very poor oxygen transmissibility. ${ }^{3}$ The second available type is called homogeneous because a normal hydrophilic lens is colored using a dyeing process. The advantages are that one can use thin, high water content lenses 4 in a large range of colors at a relative low price and acceptable delivery time. 5 With these lenses, problems reported are the lack of a sufficient opaque layer and an unnatural look when using a heavy coloring without iris pattern. With black pupil lenses patients often report that the occlusion in not total or that there is still hindering light entering the eye via the borders of the artificial black pupil. Several manufacturers have made attempts to improve the dying technique or, by the addition of an iris pattern, give the lenses a more natural look.

One of the presently available systems is subject of this study. Next to the use of a high water content material and all possible fitting parameters, the idea behind this system is to use a limited amount of colors and standardize the dimensions of iris and pupil. The aim of this study is to evaluate the clinical performance and success-rate of this system in cases where an artificial iris with clear or black pupil or a black pupil only is indicated.

\subsubsection{Material and Methods}

The study was open to patients with ocular conditions that indicated the use of iris prosthetic or occluding black pupil contact lenses. After obtaining oral informed consent from the patient, a routine ophthalmological examination was performed. Using a slit lamp measuring eye-piece, the horizontal diameter of iris and pupil was determined. In case the iris was not visible the horizontal corneal diameter was measured. K-readings were taken whenever possible and thereafter lenses were fitted according to the instruction of the manufacturer. If no K-readings could be obtained the fitting was done on basis of trial and error. For later reference a color slide was made of each and both eyes, with and without contact lenses.

All lenses were produced from a non-ionic random co-polymer based on N-Vinyl 2-pyrrolidone having a water content of $67.5 \%$. The production of the lenses involved a color dyed within the lens matrix (homogeneous type), an additional opaque backing and an iris pattern printed on the anterior surface. The individual lens color was selected from a trial set containing the five available standard colors. Standard dimension of the iris was $12.5 \mathrm{~mm}$ with a pupil diameter of $5 \mathrm{~mm}$. The system, Prosthetic Hi-colour Select manufactured by Igel International (Igel, Leighton Buzzard, UK) offers the option of a clear and black pupil combined with the prosthetic iris. Also available is a clear lens with a black dot, in diameter ranging from 5 to $10 \mathrm{~mm}$, to be used as a black occluding pupil.

At the dispensing visit the lenses were placed on the patient eye $(s)$ and evaluated with stan- 
dard procedures for fitting of soft contact lenses. The patients were instructed on care and handling of the lenses. The care system used was Unicare blue (ICN, Zoetermeer, The Netherlands), extra wetting of the lens was allowed whenever needed but solely by using unit dose $\mathrm{NaCl}$ 0.9\%.

Follow up visits were routinely scheduled at 6 weeks, 3 months, 6 months and one year after dispensing. Patients were entitled to extra visits in case adverse reactions or subjective disturbing symptoms were noticed. At initial and each follow up visit the system was evaluated using following criteria. Good fit is formulated as movement of 0.5 to $1.0 \mathrm{~mm}$ on the blink and centering such that the edge of the lens extends minimal $0.5 \mathrm{~mm}$ over the limbus in any direction including movement. Success of the lens was evaluated using the patients subjective findings in daily use and the objective examination of iris and pupil coverage. Corneal physiology was assessed and recorded following the standard procedures (CCLRU) as described by Terry ${ }^{6}$ for soft contact lenses under daily wear conditions. Loss of color with time was checked by observing the visibility of the iris or the corneal tissue behind the lens in situ using the slitlamp beam as a light source. The observations were scored on a 5 point scale representing $0,25,50,75$ or $100 \%$ loss of color. The criteria for lens replacement were $50 \%$ loss of color or patient subjective dissatisfaction wit the opaqueness of the lens.

\subsubsection{Results}

A total of 31 patients (18 females, 13 males, 34 eyes), with ocular conditions that indicated the use of an artificial iris and/or occluding black pupil, were included in this study. The age of the patients ranged from 14 to 67 years of age (average 38.4 years of age). From the 34 eyes fitted, 17 needed a prosthetic iris/clear pupil, 9 a prosthetic iris/black pupil and 8 a black pupil only. In Table 1 the indications and the type of lens used are given.

\begin{tabular}{|c|c|c|c|}
\hline Indications & $\begin{array}{c}\text { Iris prosthetic } \\
\text { Clear pupil }\end{array}$ & $\begin{array}{c}\text { Iris prosthetic } \\
\text { Black pupil }\end{array}$ & Black pupil \\
\hline Traumatic iris defect & 5 & & \\
\hline Iridectomy & 3 & & \\
\hline Iris pigment defects & 3 & & \\
\hline Iris coloboma & 2 & & \\
\hline Albino & 2 & & \\
\hline Aniridia & 2 & & \\
\hline Corneal scar & & 2 & 3 \\
\hline Corneal decompensation & & 5 & \\
\hline Diplopia & & 1 & 4 \\
\hline Non functioning eye & & 1 & 1 \\
\hline Total & 17 & 9 & 8 \\
\hline
\end{tabular}


Although the combination and the severity of signs and symptoms varied considerably among patients three main areas of indication for this type of lenses could be deduced. The first group consisted of various types of iris defects with photophobia, nystagmus, glare and hinder of incident light as signs and symptoms. In the second group lenses were fitted for cosmetic reasons in non functioning eyes due to severe pathology or trauma. The third group represented cases in which (binocular) vision was permanently disturbed, therapy had failed and only occlusion remained to relief diplopia.

Figure 1 shows an example of a case in group one (iris defects) indicating the use of a lens with an artificial iris and clear pupil. Figure 2 is an example of an artificial iris and a black pupil used for group 2 problems (cosmetic). An example of indication area 3 (disturbed vision) is given in Figure 3. The lens used had a black pupil only, with the special condition that it is also used as a bandage in a case of trichiasis.

In $78 \%$ of the eyes the standard dimensions of iris and pupil could be used. In $67 \%$ of the cases that needed a lens monocularly, one of the standard iris colors matched the fellow eye to the patients satisfaction (Table 2). The opaqueness of the prosthetic iris and the occlusion of the black pupil lenses proved at the dispensing visit to be satisfactory in all cases.

No adverse reactions were seen during the one year follow-up. The only reason for patients to appoint for extra follow ups was discoloration of the lenses and subsequent returning of
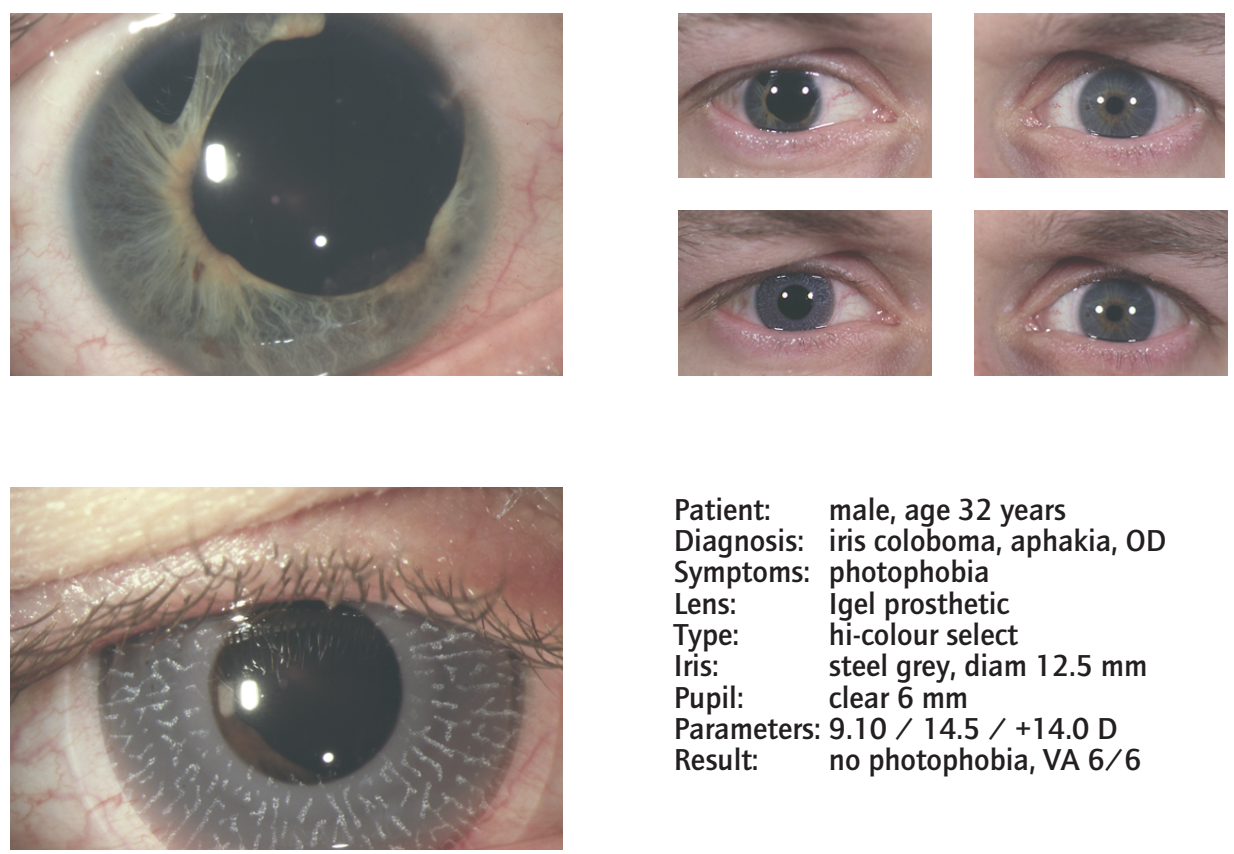

Figure 1. Photographic documentation of a patient with iris coloboma. Top left shows the condition of the (OD) eye to correct. Bottom left shows OD with the prosthetic lens. Top right shows OD and OS without (top) and with corrective lens (bottom). 

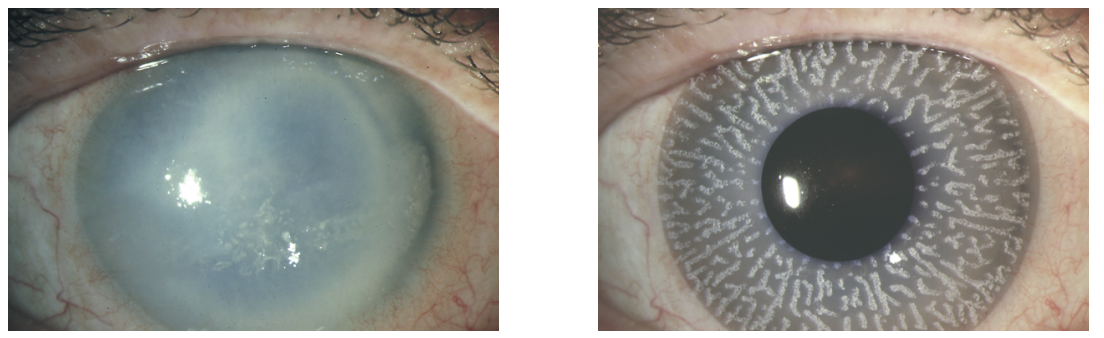

Patient: female, age 61 years

Diagnosis: corneal decompensation OD

Symptoms: cosmetic dissatisfaction

Lens: Igel prosthetic

Type: hi-colour select

Iris: $\quad$ steel grey, diameter $12.5 \mathrm{~mm}$

Pupil: $\quad$ black, diameter $5 \mathrm{~mm}$

Parameters: 8.70 / 14.5 / PLANO

Result: happy patient and doctor

Figure 2. Photographic documentation of a patient with corneal decompensation. Top left shows the condition of the (OD) eye to correct. Top right shows OD with the prosthetic lens.
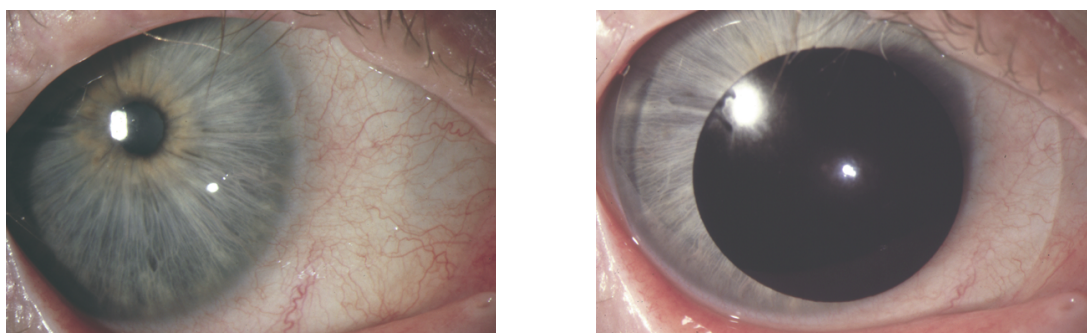

Patient: female, age 64 years

Diagnosis: trichiasis, corneal lesions, OD

Symptoms: pain, photophobia, diplopia

Lens: Igel Prosthetic

Type: totally occluding black pupil

Pupil: black, diameter $8 \mathrm{~mm}$

Parameters: 8.70 / 14.5 / PLANO

Result: no pain; healing of epithelium no incident light

Figure 3. Photographic documentation of a patient with diplopia and trichiasis. Top left shows the condition of the (OD) eye to correct. Top right shows OD with the occlusion lens. 
subjective symptoms. In combination with the criteria to replace lenses if $50 \%$ loss of color was observed this resulted in $71 \%$ of the cases to replace the lens in a range between 4 to 8 months (average at 6.5 months) after dispensing.

Two patients wear their lenses occasionally and one was discontinued due to lack of motivation.

\begin{tabular}{|c|c|c|c|c|c|c|c|c|c|c|c|c|}
\hline \multicolumn{13}{|c|}{$\begin{array}{l}\text { Results of fitting } 34 \text { eyes with iris prosthetic clear pupil, iris prosthetic black pupil and black pupil occlusion } \\
\text { contact lenses. Success is given for the use of standard lens dimensions (Dim) and for the satisfaction of the } \\
\text { patient with the cosmetic effect of the lenses (Cosm). Eyes are grouped by monocular and binocular } \\
\text { application. Percentages (bottom line) for the fitted eyes in each group are based on the total number of } \\
\text { eyes. The percentages of success for dimension and cosmetic satisfaction are related to the number of eyes } \\
\text { fitted in each group of lenses. }\end{array}$} \\
\hline \multirow{3}{*}{$\begin{array}{l}\text { Type } \\
\text { of } \\
\text { Lens }\end{array}$} & \multicolumn{3}{|c|}{$\begin{array}{l}\text { Iris prosthetic } \\
\text { Clear pupil }\end{array}$} & \multicolumn{3}{|c|}{$\begin{array}{l}\text { Iris prosthetic } \\
\text { Black pupil }\end{array}$} & \multicolumn{3}{|c|}{\begin{tabular}{|l|} 
Black pupil \\
Occlusion
\end{tabular}} & \multicolumn{3}{|c|}{ Total } \\
\hline & \multirow{2}{*}{$\begin{array}{l}\text { No. } \\
\text { Eyes }\end{array}$} & \multicolumn{2}{|c|}{ Success } & \multirow{2}{*}{$\begin{array}{l}\text { No. } \\
\text { Eyes }\end{array}$} & \multicolumn{2}{|c|}{ Success } & \multirow{2}{*}{$\begin{array}{l}\text { No. } \\
\text { Eyes }\end{array}$} & \multicolumn{2}{|c|}{ Success } & \multirow{2}{*}{$\begin{array}{l}\text { No. } \\
\text { Eyes }\end{array}$} & \multicolumn{2}{|c|}{ Success } \\
\hline & & Dim & Cosm & & Dim & Cosm & & $\operatorname{Dim}$ & Cosm & & Dim & Cosm \\
\hline Mono & 11 & 8 & 8 & 9 & 7 & 6 & 8 & 7 & 7 & 28 & 22 & 21 \\
\hline Bino & 6 & 5 & 4 & & & & & & & 6 & 5 & 4 \\
\hline Total & 17 & 13 & 12 & 9 & 7 & 6 & 8 & 7 & 7 & 34 & 27 & 25 \\
\hline$\%$ & 50 & 77 & 71 & 26 & 78 & 67 & 24 & 88 & 88 & 100 & 79 & 71 \\
\hline
\end{tabular}

\subsubsection{Discussion}

The results of our study indicate that in a systematic evaluation of cosmetic fitting in traumatized eyes, it is efficient, from a physiological standpoint, to first try standardized thin high water content colored soft lenses. With this study we can therefore conform this type of hypothesis from case reports. ${ }^{2,4}$

The use of standardized colors seems to be in accordance with the report of Golightly 5 on tinted contact lenses for non-traumatized eyes. With a limited choice of options in color it was found that patients are more easily satisfied than with the promise of exactly matching the color of the other eye as offered in hand-painted lenses.

Loss of color over time is the most important problem we have seen with this type of lenses. Loss of color varies considerably among patients. In $29 \%$ of the cases the same lens still performed satisfactory after one year whereas in other cases the lens needed replacement as early as 4 months after dispensing. It was noticed that, although with some variation, the frequency of required replacement seems to be patient related. Since all patients used the same care system (without mechanical cleaning) the reason for loss of color and the variations per patient must either be found in differences in tear composition, in variations in washing out of the dye from the lens matrix, or maybe in the variation with which patient used artificial tears. 
With the tendency of early replacement in soft contact lens wear to avoid complications in general the replacement problem is in our view only a matter of management. Information about color loss of the lenses to the patient, and the insurance company prior to the dispensing avoids problems in cost management of lens replacements.

\subsubsection{Conclusion}

The standard available high water content Prosthetic Hi-Colour Select contact lenses could be used successfully in cases where an iris prosthesis and/or a black pupil is indicated. Color matching showed to be satisfactory in $67 \%$ of the cases. Discoloration and loss of opacity was in $71 \%$ of the cases a reason to replace the lenses at average 6.5 months after dispensing. This study shows that homogeneous colored thin high water content lenses can be successfully used as a first choice option in prosthetic contact lens fitting.

\section{Acknowledgement}

We thank ICN/Oculenti and Igel International for their support in this study.

\section{References}

1. Port, M. Cosmetic and prosthetic contact lenses. Contact Lenses, chapter 21:p789-95, edited by Phillips AJ and Stone J. London, Butterworths, 1989.

2. Stechler, J. Fitting traumatised eyes with tinted and hand-painted hydrogel lenses. Optician 1991;201; part1; 5299; part2; 5304:8-29

3. Holden, B.A. and Mertz, G.W. Critical oxygen levels to avoid corneal edema for daily and extended wear contact lenses. Invest Ophthalmol Vis Sci 1984;25:1161-71.

4. Goede, H. Die Anpassung einer hoch hydrofhilen getoenten Kontaktlinse wegen aesthetischer Indikation. DOZ 1991;10:106-7 .

5. Golightly, C. How To Fit Colored Lenses More Efficiently. Contact Lens Forum 1990;18:49-53.

6. Terry, R.L. et al. CCLRU standards for success of daily and extended wear contact lenses. Optom Vis Sci 1993;70:234-43. 
Chapter 3

High Gas Permeable Rigid Contact Lenses 


\subsection{Introduction to this chapter}

This chapter is dedicated to developments in high gas permeable (HGP) CLs. With HGP CLs four subjects are of particular interest:

1. Development of materials and production techniques.

2. Clinical testing of new aspheric CL designs and fitting techniques.

3. Evaluation of safety and efficacy of care systems.

4. Study of the effects of high gas permeable CLs on corneal physiological functioning.

Production techniques, material selection and lens design of rigid gas permeable (RGP) CLs were the subject of intensive research during the 1980s. In the 1970s materials having some permeability to gasses, like Poly(4-methylpent-1 ene) known as TPX ${ }^{1}$ and Cellulose Acetate Butyrate $(\mathrm{CAB})^{2}$, were introduced. Early work by Larke ${ }^{3}$, Pedley ${ }^{4}$, Tighe ${ }^{5}$ and $\mathrm{Ng}^{6}$ showed a need to optimise stability, wettability and oxygen permeability in relation to thickness and lens design in order to make these materials suitable for prescribing on a large scale. This resulted in the introduction of the siloxymethacrylate - and fluoro-polymers in the 1980s. Initially CLs produced from these new materials showed many problems, like instability of base curve, deposit formation and breakage. ${ }^{7-9}$ The implementation of improvements in manufacturing technique could greatly reduce these problems. ${ }^{10}$ However, despite evidence that diminishing hypoxia would be very beneficial to corneal physiology ${ }^{11}$, some hesitation in prescribing HGP CLs remained. ${ }^{8}$

During the same time period renewed attention was given to better lens - corneal alignment with the introduction of aspheric lens designs. ${ }^{12}$ The initial problem with these improved lens designs was that, due to heavy polishing as was common in classically manufactured CLs, the shape could not be controlled satisfactory. A further complication has been the fact that CLs with an aspheric design over the entire posterior lens surface showed a different behaviour on the eye than spherical lenses. ${ }^{13,14}$ Therefore modern aspheric CLs use a spheroasphere design to overcome problems in optics (Kok ${ }^{15}$ page 18-9) and centration. ${ }^{16,17}$ The advancements in material, manufacturing and lens designs has directed our research to comparing the performance of CLs with high and low gas permeability in both spherical and sphero-asphere design. This study is described in section 3.2 of this thesis.

Parallel to the development of modern aspheric CL designs, advancements are also made in measuring techniques of the cornea. Topometry uses a classical keratometer with a special fixation device and enables the practitioner to incorporate extra information from four sagittal measurements of the corneal periphery. ${ }^{18,19}$ Unfortunately it appears that the advancements of these techniques for corneal topography, including photokeratoscopy ${ }^{20}$ have not been accepted generally as new standard methods to predict CL parameters. One of the reasons is certainly that practitioners were initially confused by the early problems in HGP CL manufacturing. Since the improvements in manufacturing techniques for high gas permeable CLs made it possible to produce lenses with stable and reproducible parameters ${ }^{21}$, renewed attention is given to advanced fitting techniques 22,23 and the appropriate care of HGP CLs. ${ }^{24-26}$ These items are the subject of study in section 3.3 of this thesis. 


\section{References}

1. Kamath P. Physical and chemical attributes of an ideal contact lens. Contacto 1969;13:29-34.

2. Stahl N, Reich L, Ivani M. Report on laboratory studies and preliminary clinical application of a gaspermeable plastic contact lens. J Am Optom Ass 1974;45:302-07.

3. Larke J, Pedley D, Tighe B. Polymers in contact lens applications: Parts 3 and 4. Wettability phenomena. Optician 1973;166:21-30:32-9.

4. Pedley D. Hydrophilic polymers. Birmingham, University of Aston, 1976.

5. Tighe B. The design of polymers for contact lens applications. Br. Polymer J. 1976;8:71-7.

6. Ng C. Polymers in contact lens applications: Oxygen permeability and surface hydrophilicity of poly(4methylpent-1-ene) and related polymers. Br Polymer J 1976;8:124-30.

7. Walker J. Comparing the base curve stability of various contact lens materials. Contact Lens Forum 1989;March:59-69.

8. Walker J. High Dk RGPs are the lenses of first choice. Optician 1992;Febr 7:18-25.

9. Schwartz C. Contact lenses in the 1990s: fitting for lifestyles. Contact Lens Forum 1990;Jan:17-29.

10. Proctor E. Contact lens manufacturing. Contact Lenses, Ed. 3, Edited by Phillips A, Stone J. London, Butterworths, 1989

11. Holden BA, Mertz GW. Critical oxygen levels to avoid corneal edema for daily and extended wear contact lenses. Invest Ophthalmol Vis Sci 1984;25:1161-7.

12. Hirst E. Fitting a true aspherical contact lens. Contacto 1974;1:15-9.

13. Ames KS, Erickson P. Optimizing aspheric and spheric rigid lens performance. CLAO J 1987;13:165-70.

14. Forst G. Comparison of the movement of contact lenses with spherical and aspherical back surfaces. Contact Lens Journal 1990;18:129-32.

15. Kok J. New developments in the field of contact lenses. Thesis, University of Amsterdam, The Netherlands, 1991. ISBN 9060763394.

16. Ames K, Jones W. Spherical versus aspherical designs: A clinical difference? Contact Lens Forum 1988:18-22.

17. Jones L, Jones D. A new high Dk elliptical lens. Optician 1992;Jan 10:16-23.

18. Wilms K, Rabbetts R. Practical concepts of corneal topometry. Optician 1977;Sept 16:7-13.

19. Wilms K. Ueberlegungen zur Topometrie von Cornea und Kontaktlinsen. Neues Optik J 1980;15:569-71.

20. Guillon M, Lydon DP, Wilson C. Corneal topography: a clinical model. Ophthalmic Physiol Opt 1986;6:47-56.

21. Brabander de J, Brinkman CJJ, Kok JHC, Mil van C. Low or high Dk? Spherical or aspherical rigid gas permeable lenses? The gap between theory and practice. J BCLA 1992;15:159-62.

22. Huppertz H. Grundkonzeption und Anpaszphilosophie der asphaerischen Kontaktlinsen. Kontaktlinse 1983:15-30.

23. Goldberg J. Basic principles of aspheric corneal lenses. Contact Lens Forum 1988:35-8.

24. Terry R, Schnider C, Holden BA. Rigid gas permeable lenses and patient management. CLAO J 1989;15:305-9.

25. Begley CG, Weirich B, Benak J, Pence NA. Effects of rigid gas permeable contact lens solutions on the human corneal epithelium. Optom Vis Sci 1992;69:347-53.

26. Vanhakendover C. [Care of contact lenses]. Bull Soc Belge Ophtalmol 1993;247:8-11. 


\subsection{HGP Contact Lenses, Material and Design}

Low or High Dk? Spherical or Aspherical Rigid Gas Permeable Lenses? The Gap between Theory and Practice

John de Brabander, Cees J.J. Brinkman, Jan H.C. Kok and Cor J. van Mil.

From:

The Cornea and Contact Lens Unit, Oculenti Practice at the Department of Ophthalmology, Academic Medical Centre, University of Amsterdam, the Netherlands.

J BCLA 1992;15;4:159-62. 
Abstract

Purpose. The aim of the research described in this article is to quantify the current situation with respect to the clinical functioning of high or low Dk materials and spherical or aspherical lens designs. In our research we sought significant differences between four lens groups in terms of overall success, fitting characteristics, physiological functioning, back curve optical radius (BCOR) stability, and lens condition.

Methods. The 68 patients that participated were randomly divided into four groups, which were then fitted with a combination of high (EQUA II) or low (RXD) Dk materials and spherical or aspherical lens design. The initial BCOR selection was based on central K-readings, with compensation for corneal flattening at $30^{\circ}$ (E-value). Follow-up examinations were performed at 6 weeks, 3 months, 6 months, and 1 year after dispensing the lenses. At the first follow-up visit ( 6 weeks), the fit was evaluated against the criteria of perfect corneal alignment at the flattest meridian, good centring, adequate movement, and an edge clearance which gave proper tear circulation without air bubbles being trapped or debris. If these criteria were not met, the fit was first improved by changing the BCOR for the same lens design, second by switching to the other design. At all follow-up visits the eyes and lenses were examined with a slit lamp using routine clinical procedures. If any significant problem occurred the first option was to change the material, but keep the earlier optimised geometry. Also K-readings were taken and the BCORs of the lenses were measured using a radiuscope.

Results. The overall success rate was 77\% after one year follow up. In $11 \%$ of the cases the wearing time was limited due to dryness symptoms. In $12 \%$ of the cases wear was discontinued within 6 months mainly related to discomfort. In fitting characteristics the spherical lenses were changed to asphericals in 10 eyes compared to 4 in which the aspherical lenses were changed to sphericals. These changes were not related to the material. Concerning physiological functioning no serious adverse reactions were found. In 15 eyes (11\%) mild, but persistent 3-9 o'clock staining was observed. Changing to lenses of other design or material could reduce the symptoms in only 4 eyes. The stability of the BCOR ranged from $-0.08 \mathrm{~mm}$ to $+0.06 \mathrm{~mm}$ from the baseline value (average -0.0012 , SD 0.02 ) without statistically significant differences between the four lens groups. The evaluation of lens condition showed deposits on 12 lenses that could be solve by extra cleaning; superficial scratches on 9 lenses without related symptoms; during the 1 year wearing time, breakage occurred in five lenses. Lens conditions were not related to material or lens design.

Conclusion. HGP lenses can be fitted with good success in both spherical and aspherical design. Both materials tested (RXD and EQUA II) gave good results with respect to lens condition and geometrical stability.

\section{Key words:}

$\mathrm{Dk}$, design, aspherical contact lenses. 


\subsubsection{Introduction}

Rigid gas permeable (RGP) contact lenses were introduced some 15 years ago. Since that time the oxygen permeability $(\mathrm{Dk})$ has increased from around 10 for the early materials up to 150 or more for the modern high gas permeable (HGP) materials. During the 15 years of development, research has focused on the condition of the cornea with respect to oxygenation. ${ }^{1}$ It has been shown clearly that the highest $\mathrm{Dk}$ possible would be most beneficial to corneal physiology. ${ }^{2}$ One would thus expect that the majority of today's rigid contact lens prescriptions would be a HGP lens, but this is not the case. Sales figures ${ }^{3}$ in most countries show less than $50 \%$ of lenses prescribed are RGPs, and HGPs only account for around $14 \%$ (Fig. 1). There seems to be a gap between theory and practice. ${ }^{4} \mathrm{~A}$ similar situation exists in the use of aspherical versus spherical contact lenses. An aspherical posterior lens geometry has undoubtedly better corneal alignment properties. ${ }^{5}$ And yet still the majority of rigid lenses have classical multi-curve designs. Problems encountered with first generation true aspherical lenses were decentration, debris, and lens binding. ${ }^{6}$ A combination of a HGP material with an aspherical lens design would be the first starting point in optimising the lens-corneal relationship and physiological condition. Strangely enough, the sales of these lenses are lower than most. Again, theory and practice are not correlated.

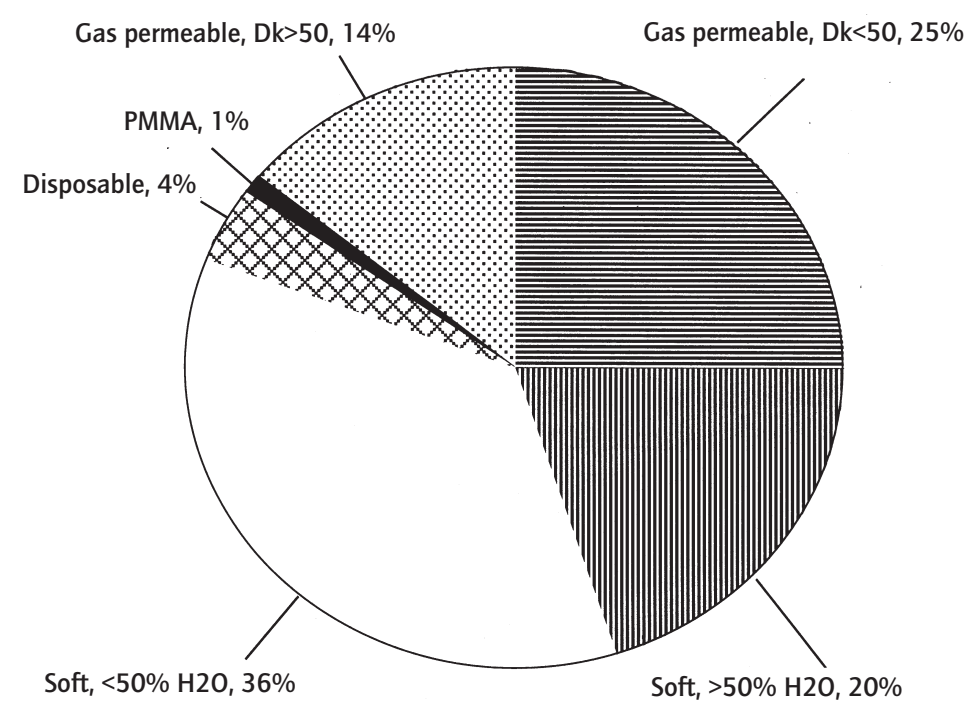

Figure 1. Sales figures for contact lenses in the Netherlands, 1990-1991.

\section{The First Choice Problem}

There are a number of arguments used to explain the current discrepancy between the theoretical lens of first choice and that made in daily practice. The foremost argument is that HGPs have provided a number of practical complications. There are many studies that clear- 
ly show problems with HGP materials, including changes in base curve, deposits, plaque formation, scratches, cracking and breakage. ${ }^{3,4,7}$ There is strength in the argument that a high Dk lens with a poor surface is worse than a low Dk lens with good wettability. One could even argue that polymethylmethacrylate (PMMA) lenses are better than HGPs with poor surfaces. With these problems, it is clear why practitioners have become reluctant to fit HGP lenses as their first choice.

A second argument that could be used against the use of high Dk lenses lies within the theory itself. From the classic concept of equivalent oxygen percentage (EOP) curves, it is not difficult to show that there is no need for high $\mathrm{Dk}$ lenses under daily wear conditions. Here we encounter a problem of definition. One could say that the minimum required $\mathrm{Dk}$ is around 30 in daily wear. But what is daily wear? Are we willing to assume that patients never nap with their lenses in or that they never sleep with them in? We must account for modern life, with discotheques, house parties and late night passion. Today's patients easily run into extended wear situations and then the need for high Dk is obvious.

A third argument is price. Raw materials in the high Dk range are more expensive and the production of these lenses demands high-technology skills and machinery. This results, together with the shorter life span of such lenses, in higher costs for the patients.

All these arguments taken together lead to the situation in which high Dk lenses are not used as lenses of first choice. Practitioners who debate on this subject usually take one of two standpoints. 'High Dk aspherical lenses are the best' or the opposite opinion 'if you want problems fit high $\mathrm{Dk}$ aspherical lenses'. The aim of the research described in this article is to quantify the current situation with respect to the clinical functioning of high or low $\mathrm{Dk}$ materials and spherical or aspherical lens designs. In our research we sought significant differences between four lens groups in terms of overall success, fitting characteristics, physiological functioning, back curve optical radius (BCOR) stability, and lens condition.

\subsubsection{Materials and Methods}

We used 68 patients in this study. They all had normal healthy eyes and had not worn lenses before. The patients were randomly divided into four groups, which were then fitted with a combination of high (EQUA II) or low (RXD) Dk materials and spherical or aspherical lens design.

The spherical lenses have an $E$-value of 0 at $30^{\circ}$, and the $E$-value of the aspherical lenses at $30^{\circ}$ is 0.4 . Both spherical and aspherical lenses have fixed axial edge lifts of $0.10 \mathrm{~mm}$ and the same centre thickness-power-diameter relation. All lenses were produced by Oculenti Laboratories, using the latest $\mathrm{CNC}$ lathe technique. The physical properties of the materials are given in Table 1 .

The initial BCOR selection was based on central $K$-readings, with compensation for corneal flattening at $30^{\circ}$ ( $E$-value). Follow-up examinations were performed at 6 weeks, 3 months, 6 months, and 1 year after dispensing the lenses.

At the first follow-up visit ( 6 weeks), a careful fitting evaluation was performed by two investigators separately and without consultation. Criteria for best fit were perfect corneal align- 


\begin{tabular}{|c|c|c|}
\hline Physical properties & RXD 45 & EQUA II 125 \\
\hline$D k$ (Fatt units, $\left.35^{\circ} \mathrm{C}\right)$ & 45 & 125 \\
\hline Wetting angle & $39^{\circ}$ & $30^{\circ}$ \\
\hline Hardness (Shore) & 122 & 113 \\
\hline Saline absorption & $<1.0 \%$ & $<1.0 \%$ \\
\hline Refractive index & 1.435 & 1.423 \\
\hline Specific gravity & 1.27 & 1.24 \\
\hline
\end{tabular}

ment at the flattest meridian, good centring, adequate movement, and an edge clearance which gave proper tear circulation without air bubbles being trapped or debris. If these criteria were not met the fit was first improved by changing the BCOR for the same lens design. If this result did not meet the criteria, the patient was refitted with a lens of the other design.

At the follow-up visits the eyes and lenses were examined with a slit lamp using routine clinical procedures. If any significant problem occurred the first option was to change the material, but keep the earlier optimised geometry. Also $K$-readings were taken and the BCORs of the lenses were measured using a radiuscope.

All patients were given the same instruction and all used Unicare Green (produced by ICN Pharmaceuticals, Holland) for insertion and daily storage plus Ocuclean (supplied by Oculenti, Holland) for cleaning if desired.

\subsubsection{Results}

\section{Overall Success}

From the 68 patients that were initially fitted, eight (12\%) discontinued wear within 6 months. Table 2 gives the reasons for failure. We were unable to overcome these problems with lenses of other design or material. Of the remaining 60 patients (120 eyes), 52,5

\begin{tabular}{|c|c|c|c|c|c|}
\hline $\begin{array}{l}\text { Discontinued } \\
\text { within } 6 \text { months }\end{array}$ & & $\begin{array}{l}\text { Limited wear } \\
\text { after } 1 \text { year }\end{array}$ & & $\begin{array}{l}\text { Successful wear } \\
\text { after } 1 \text { year }\end{array}$ & \\
\hline Reason & No. & Reason & No. & Criterion & No. \\
\hline Motivation & 2 & 3-9 o'clock stain & 1.5 & Wearing lenses & \\
\hline Discomfort & 2 & Surface dry & 4 & all waking hours & 52.5 \\
\hline Wearing time & 2 & Wearing time & 2 & & \\
\hline \multirow[t]{2}{*}{ Intolerance } & 2 & & & & \\
\hline & $8(12 \%)$ & & $7.5(11 \%)$ & & $52.5(77 \%)$ \\
\hline
\end{tabular}


patients (105 eyes) wore the lenses for longer than 12 months without problems. Table 2 also details the persistent symptoms that occurred with limited wear in 15 eyes. These symptoms could not be diminished by changing to another material or lens design, but after the 1 -year follow-up there was no reason to discontinue lens wear.

\section{Fitting Characteristics}

After careful evaluation it was found that in ten eyes (8.3\%) with spherical lenses changing the BOCR could not further improve the fit (Table 3). Typically, in such cases the tear layer is slightly flat in the centre, but the periphery shows a nearly bearing area. Only four out of the ten eyes had symptoms related to wearing time. Changing to the spherical design showed an improvement in all ten cases.

In the group wearing aspherical lenses, four eyes (3.3\%) showed a nearly steep centre, but also too much edge clearance. In this group, all eyes had problems with comfort. Changing from aspherical to spherical lens design could improve the fit in all of these eyes. All of the differences in fitting characteristics are statistically not significant and not related to the material (Chi-square, $p \leq 0.5$ ).

\begin{tabular}{|c|c|c|c|}
\hline RXD 45 & & EQUA II 125 & \\
\hline Spherical: & & Spherical: & \\
\hline Change fit & 6 & Change fit & 4 \\
\hline Limited wear & 0 & Limited wear & 3 \\
\hline Partial dry plus 3-9 o'clock staining & 2 & Partial dry plus 3-9 o'clock staining & 4 \\
\hline Good wetting plus 3-9 o'clock staining & 2 & Good wetting plus 3-9 o'clock staining & 0 \\
\hline Aspherical: & & Aspherical: & \\
\hline Change fit & 2 & Change fit & 2 \\
\hline Limited wear & 3 & Limited wear & 2 \\
\hline Partial dry plus 3-9 o'clock staining & 2 & Partial dry plus 3-9 o'clock staining & 2 \\
\hline Good wetting plus 3-9 o'clock staining & 1 & Good wetting plus 3-9 o'clock staining & 2 \\
\hline
\end{tabular}

\section{Slit-lamps findings}

In none of the cases were serious adverse reactions found. The results from the 120 eyes showed three groups of problems - limited wear, partial dryness with 3-9 o'clock staining, and 3-9 o'clock staining with good wetting of the lens. Table 3 shows that these findings are not related to the lens design. Limited wearing time occurred in eight eyes. Typical symptoms in this group were dry irritating eyes with troublesome vision at the end of the day. No particular slit-lamp signs were found. All changes in material or lens design appeared negative. Patients were advised to blink properly and to rewet their lenses.

Partial dry surfaces associated with 3-9 o'clock staining were found in four RXD lenses and in six EQUA II lenses. Replacing the RXD with EQUA II lenses did not solve the problem. 
Replacing EQUA II with RXD lenses did solve the problem in two out of six eyes.

Good surface wetting but with 3-9 o'clock staining was present in three aspherical and in two spherical lenses. Replacing aspherical with spherical lenses solved the problem in two out of the three aspherical lens wearers. Replacing spherical with aspherical lenses was not successful.

At the end of the study all those with 3-9 o'clock staining were refitted with hyper-thin lenses of large diameter. In six out of the 15 eyes the problem was solved by this method.

\section{Lens Condition}

Deposits were found in 12 eyes (Table 4), but there was no relation with materials or lens design. The patients could satisfactorily solve the problem by extra cleaning.

Superficial scratches were seen in four RXD and in five EQUA II lenses, but the patients had no related symptoms. During the 1 year wearing time, breakage occurred in three RXD and two EQUA II lenses.

\begin{tabular}{|c|c|c|c|}
\hline RXD 45 & & EQUA II 125 & \\
\hline Spherical: & & Spherical: & \\
\hline Deposits, mild & 2 & Deposits, mild & 4 \\
\hline Scratches, light & 3 & Scratches, light & 3 \\
\hline Breakage & 1 & Breakage & 2 \\
\hline Change in BCOR: & & Change in BCOR: & \\
\hline Average & -0.002 & Average & -0.001 \\
\hline Range & -0.04 to +0.03 & Range & -0.08 to +0.04 \\
\hline Aspherical: & & Aspherical: & \\
\hline Deposits, mild & 3 & Deposits, mild & 3 \\
\hline Scratches, light & 1 & Scratches, light & 2 \\
\hline Breakage & 2 & Breakage & 0 \\
\hline Change in BCOR: & & Change in BCOR: & \\
\hline Average & -0.0035 & Average & +0.003 \\
\hline Range & -0.04 to +0.06 & Range & -0.04 to +0.04 \\
\hline
\end{tabular}

\section{BCOR Stability}

The measurements of BCOR stability showed (Table 4 ) an overall range of $-0.08 \mathrm{~mm}$ to $+0.06 \mathrm{~mm}$ from the baseline value, with an average of -0.0012 (SD 0.02). There was no statistically significant difference between the four lens groups in BCOR stability. 


\subsubsection{Conclusions}

- HGP lenses can be fitted with good success.

- Spherical and aspherical lens designs' performances were not significantly different.

- Both materials tested gave good results with respect to surface problems and stability.

- The remaining problems in wearing HGP lenses are dryness of the lens surface and 3-9 o'clock staining. These problems are not related to lens design or material.

\subsubsection{Discussion and Recommendations}

Our results show no significant differences in performance between the two lens designs or materials used. It should be remembered that our patients wore the lenses on a daily-wear basis and all our measurements were made using routine clinical procedures. That there is no difference in performance shows that with well-manufactured lenses the first choice material can be that with the highest Dk. The use of aspherical versus spherical lens designs is currently a matter of taste; however, we believe that with the advent of automated cornea measuring techniques the aspherical lenses will become first choice in the future. Finally, we hope that the gap between theory and practice will soon be eliminated.

\section{References}

1. Holden BA, Mertz GW. Critical oxygen levels to avoid corneal edema for daily and extended wear of contact lenses. Invest Ophthalmol Vis Sci 1984;25:1161-7.

2. Kok JHC, Vreugdenhil W. Extended wear high oxygen permeable Boston Equa and Fluoroperm Contact Lenses. Contactologia 1990;12:163-7.

3. Walker J. High Dk RGPs are the lenses of first choice. Optician 1992;Feb 7:18-25.

4. Schwartz CA. Contact lenses in the 1990s: fitting for lifestyles. Contact Lens Forum 1990;Jan:17-29.

5. Jones L, Jones D. A new high Dk elliptical lens. Optician 1992; Jan 10:16-23.

6. Forst G. Comparison of the movement of contact lenses with spheric and aspheric back surfaces. Contact Lens J 1989;18;5:129-33.

7. Walker J. Comparing the base curve stability of various contact lens materials. Contact Lens Forum 1989;March:59-69. 


\subsection{HGP Contact Lenses, Fitting and Care Systems}

Evaluation of a New System of Lens Parameter Selection and Comparison of Traditional vs One-Step Lens Care Systems for Aspheric High-Dk RGP Contact Lenses

John de Brabander ${ }^{1,2}$, Jan H.C. Kok ${ }^{3}$, Rudy M.M.A. Nuijts ${ }^{1}$.

From:

1 The Eye Research Institute Maastricht, Department of Ophthalmology, University Maastricht, the Netherlands.

2 The Contact Lens Unit, Oculenti Practice, Department of Ophthalmology, Academic Medical Center of the University of Amsterdam, the Netherlands.

3 Medical Center Jan van Goyen, Amsterdam, the Netherlands.

The CLAO Journal 2000;26;4:193-99. 
Abstract

Purpose. We investigated the value of newly developed systems for parameter selection on the performance of high Dk rigid gas permeable (RGP) contact lenses with a spheric-aspheric design made of Boston Equa material in normal daily wear conditions. A comparison was also made between the safety and efficacy of a traditional care system vs a one-step chemical care system during the follow-up period of 1 year.

Methods. A total of 66 patients were randomly assigned to traditional and newly developed systems for computing the back optical zone radius (BOZR) and the total diameter (TD) of the lenses. Evaluation parameters incorporated assessment of fit, visual acuity, wearing time, comfort and lens condition.

Results. The traditional method of computing the BOZR, using a method based on flat-K, predicted the BOZR successfully in $83 \%$ of the cases. Corneal topometry reached a lower, but not significantly different $(\mathrm{P}>0.01)$ value of $75 \%$ correct prediction of BOZR. Analysis of the differences in eccentricity $(\mathrm{E})$ values between quadrants per eye was a significant $(\mathrm{P}<$ $0.001)$ factor in fault predictions in the topometric system. A newly developed guide for TD selection performed, with $90 \%$ correct predictions, significantly $(\mathrm{P}<0.01)$ better than a traditional method $(72 \%)$ based on corneal diameter on supraversion. No significant differences $(\mathrm{P}>0.05)$ were found in the capabilities of care systems (a traditional care system using surfactant cleaner vs a one-step solution) to keep the lenses in proper condition and without deformation, over the 1 year follow-up period. The final success rate was $85 \%$.

Conclusions. Although high Dk RGP contact lenses can be fit and used with a $85 \%$ success rate using advanced systems for determining the BOZR and TD, it remains necessary to evaluate the fit before dispensing high Dk RGP lenses in 15\% of the cases. The one-step solution used in this study was a good starting point for lens care. If needed, surfactant cleaning in the traditional care system can be done without the risk of lens deformation.

\section{Key words:}

Aspherical HGP contact lenses, contact lens fitting, BOZR selection, DIAM selection, rigid contact lens care systems. 


\subsubsection{Introduction}

Almost simultaneously with the introduction of high Dk rigid gas permeable (RGP) contact lens materials, the use of aspheric back surface lens designs ${ }^{1,2}$ and new fitting methods that incorporate more information on corneal shape ${ }^{3,4}$ have become more common. Kok and coworkers 5,6 have proven that such a combination can be of great value in prescribing high Dk RGP contact lenses in special cases like keratoconus. This has again raised the question of the feasibility of a direct ordering system for rigid contact lenses. ${ }^{7}$ Such a system would avoid or decrease the time factor in the currently used "trial and error" method of contact lens fitting. The predictive value for such a system must be sufficient to avoid the development of serious complications.

The introduction of high Dk RGP contact lens materials represents a breakthrough in the biocompatibility of contact lenses. ${ }^{8,9}$ In recent years the emphasis in material development has shifted to high gas permeability. At the same time, there has been some confusion about the validity of the units in which gas permeability is expressed (Dk value) as the methods to measure this parameter have evolved and changed over the years. Today, a Dk value of 60 (although still somewhat arbitrary) is often used to discriminate between low and high gas permeability. High Dk lenses are mostly used in special cases or in cases in which extended or occasionally overnight wear is indicated. ${ }^{10}$ Breakage, deposit formation and deformation are some of the reasons why practitioners may hesitate to prescribe contact lenses with high oxygen permeability for normal correction of ametropia under daily wear conditions. ${ }^{11,12}$ Recent research ${ }^{13}$ has shown that high Dk RGP lenses can be successfully fitted, but problems remain with dryness of the lens surface and 3-9 o'clock staining. In this respect the particular care system in use may be an important factor.

Traditional or one-step chemical cleaning systems are used to care for high Dk RGP lenses. In traditional care systems deposits are removed daily and cleaned digitally with a special surfactant cleaning solution. The lenses are stored overnight in a special disinfecting solution (soaking solution) and inserted with an other specially formulated solution (wetting solution). However, it has been found that these relatively complex systems inherently rely on patient compliance. Intomart ${ }^{14}$, a consumers' survey institution in The Netherlands, found that more than $50 \%$ of the patients that started with a contact lens care system containing more than one solution did not use it anymore after 1 year. Also, there is a risk of lens deformation with digital cleaning in the traditional cleaning/disinfection system. This led to attempts to formulate so called one-step solutions containing chemical cleaners that are able to perform all tasks in one formulation. The cleaning action is performed by chemical agents during storage overnight.

The first aim in this study was to compare new methods with traditional methods in predicting the back optical zone radius (BOZR) and the total diameter (TD) of aspherical high Dk RGP contact lenses. Using the results, the feasibility of a direct ordering system was tested.

The second aim in this study was to compare the safety and efficacy of a specially formulated one-step system for high Dk RGP contact lenses with a system containing separate solutions for traditional cleaning and wetting/storage. 


\subsubsection{Material and Methods}

In this study, 66 patients were fit with Exel (Oculenti, Zoetermeer, The Netherlands) high Dk RGP contact lenses (Fig. 1). The back surface of the lens has a spheric-aspheric design. The spherical BOZR continues as a progressive asphere from point $\mathrm{x}$. This point, the end of the back optical zone diameter (BOZD) is placed in relation to the BOZR value and TD of the lens. The aspheric back peripheral radius (BPR) also links the end of the BOZR (x) to the edge of the lens so that for a given TD/BOZR combination an ideal radial edge lift (REL) is determined. All lenses used in this study were composed of Boston Equa material (Table 1).

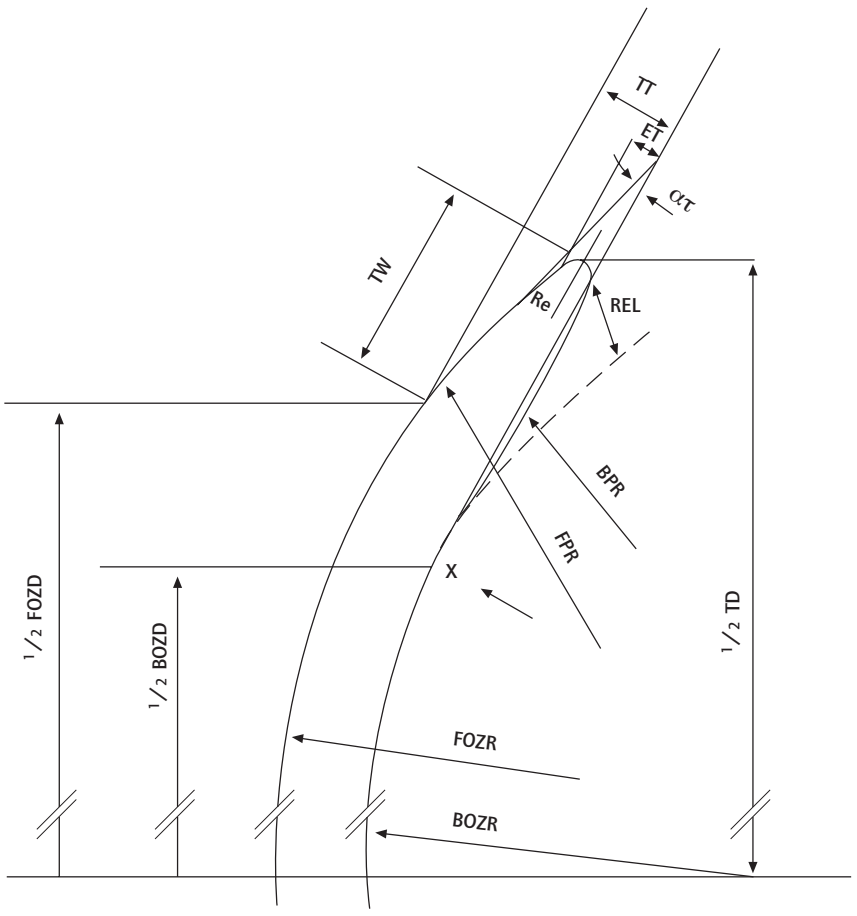

Figure 1. Design of spheric-aspheric Exel high Dk RGP contact lens

Patients were recruited among personnel and students of the Academic Medical Center, Amsterdam, the Netherlands and entered the study after giving their informed consent. Intake criteria were an age between 15 and 40 years, no presence or history of eye pathology, refractive sphere equivalent power between -6.00 and $+4.00 \mathrm{D}$, corneal astigmatism less than $3.00 \mathrm{D}$ and no contact lens wear 6 months prior to the study.

Prior to fitting each eye, a randomly selected fitting instruction was made known to the investigators. This instruction contained the information on what method to use for the 


\begin{tabular}{|c|c|}
\hline Material type & Fluor carbon / acrylate \\
\hline Oxygen permeability & $71 \times 10^{-11}(\mathrm{Dk})$ \\
\hline Wetting angle & 38 (captive bubble technique) \\
\hline Refractive index & 1.439 \\
\hline Hardness & 111 (Rockwell) \\
\hline Light transmission & $95 \%$ \\
\hline Absorption & $1 \%$ (saline) \\
\hline
\end{tabular}

selection of the BOZR, the TD, and also the care system to prescribe. For each of these variables there was a choice between a traditional and a newly developed method. Detailed descriptions of these methods follow.

BOZR selection was based on a traditional central flat-K method (Table 2) or a corneal topometric method. The latter method, originally developed by Wilms ${ }^{3,4}$, incorporates next to central flat and steep K-readings four sagittal measurements at $30^{\circ}$ along the principle meridians. The $30^{\circ}$ displacement of the eye is reached by asking the patient to fixate special marks on the keratometer. From the measured six K-readings, the corneal eccentricity (E) in each semi-meridian was calculated. Using the average E-value of the cornea, the BOZR of the contact lens was computed based on the principle of minimal tear layer volume. ${ }^{10}$ For purpose of comparison, all eyes were measured by both methods. We hypothesized that the topometric method would predict the BOZR significantly better than the flat-K method.

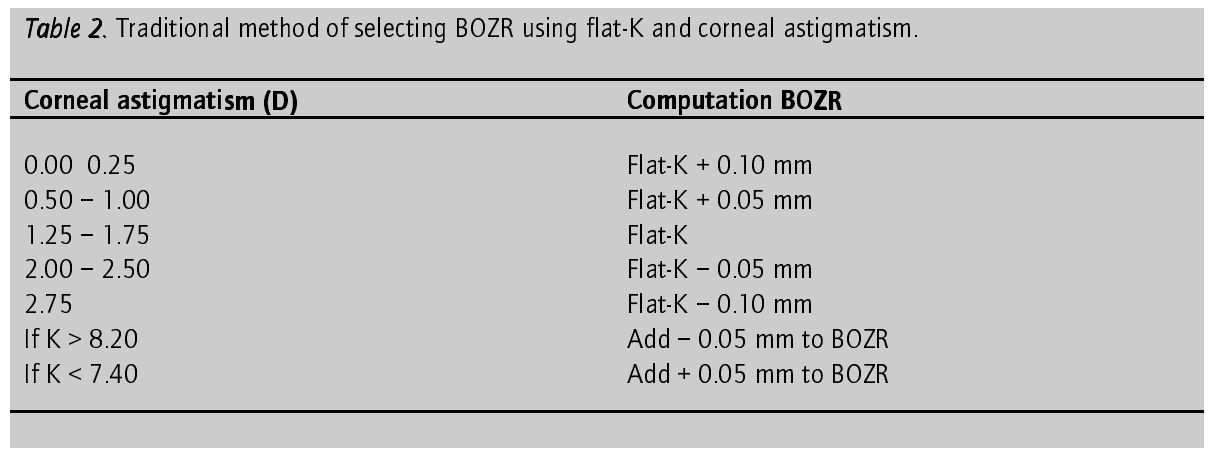

Diameter selection was based on: 1) a traditional supraversion method in which one uses the vertical visible iris on supraversion as the lens TD; or 2) a newly developed method called Exel diameter selection guide (Fig. 2). This guide has been composed after a retrospective evaluation of lens diameters used in relation to flat corneal radii, visible iris diameters, and additional eye parameters from a large pool of successful rigid contact lens wearers. Consequently the guide uses flat K-reading and vertical visible iris diameter (VVID) as the major starting point for TD selection. Other measurements of the eye are used as addition- 
Exel Diameter Selection Guide

Flat-K

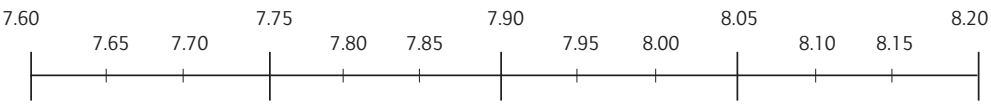

Exel

diam.

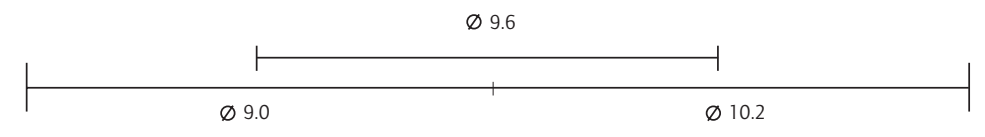

WID
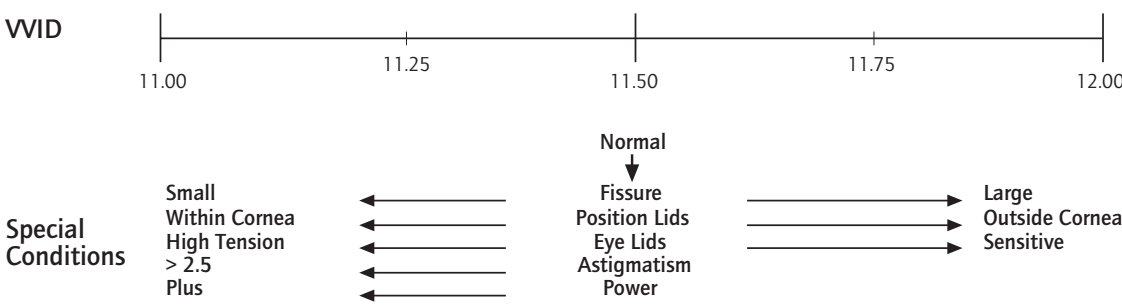

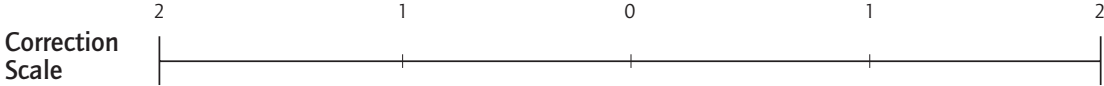

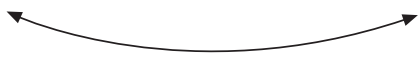

Figure 2. Exel diameter selection guide: Connect with a slide rule the flattest keratometric value (flat- $K$, top line) and the vertical visible iris diameter (WID, third line). Correct for special conditions (see bottom part) by fixing the slide rule on flat- $K$ and move it at the bottom, depending on the special condition, to the right or left. The correction scale can be used as a measure for the desired amount of correction related to the assessed severity of the special condition present. Note that more than one condition may be present in an eye - the final result is cumulative. Finally read the proposed diameter at the middle line.

al factors. The use of the guide is explained in the legend of Figure 2. For purpose of comparison, all eyes were measured according to both methods. We hypothesized that the selection guide method would predict the TD of the lens significantly better than the supraversion method.

The assigned care systems involved a traditional method including a surfactant cleaner (Boston Ocuclean) and a solution for wetting and soaking (Ocuhard) or a one-step solution including a chemical cleaning agent (Unicare green). The composition of these solutions is given in Table 3. We hypothesized that the surfactant cleaner would keep the lenses significantly cleaner than 


Table 3. Composition (volume percentages) of solutions in the used care systems. Note composition is the
same for wetting and disinfecting components; there is an additional chemical cleaning agent in the one-step
solution. All solutions are produced by ICN, Pharmaceuticals, Zoetermeer, the Netherlands.
\begin{tabular}{ll} 
Ocuhard (wet and soak) & Unicare green (one-step) \\
\hline & Cocamidopropyl PG-dimoniumphosphate $0.03 \%$ \\
EDTA $0.1 \%$ & EDTA $0.1 \%$ \\
SF $881.2 \%$ & SF $881.2 \%$ \\
Viscose forte $0.5 \%$ & Viscose forte $0.5 \%$ \\
Aqua & Aqua \\
& Polaxamer ICN \\
\end{tabular}

the one-step solution, but due to the lack of mechanical action, the one-step chemical method would keep the lenses in significantly better in shape (less changes in BOZR).

At the initial visit, lens parameters were chosen following the assigned procedure. The lenses

\begin{tabular}{|c|c|c|}
\hline Subject & Criterion & Description \\
\hline Movement & $\begin{array}{l}\text { Superior limbus to inferior eye } \\
\text { lid: smooth and continuous }\end{array}$ & On the blink \\
\hline Centration & $>0.5 \mathrm{~mm}$ & $\begin{array}{l}\text { Position of lens edge within the limbus and } \\
\text { beyond the pupil (dilated) in all directions } \\
\text { including movement on the blink }\end{array}$ \\
\hline Static fluorescein pattern & $\begin{array}{l}\text { Parallel fit with distinct edge } \\
\text { clearance }\end{array}$ & $\begin{array}{l}\text { Slitlamp, blue filter, low magnification and } \\
\text { frontal position. Fluorescein just visible in the } \\
\text { central area, distinct fluorescence at the edge }\end{array}$ \\
\hline Post-lens tear film profile & $\begin{array}{l}\text { Parallel tear layer with distinct } \\
\text { edge clearance }\end{array}$ & $\begin{array}{l}\text { Slitlamp, cross section with fluorescein, high } \\
\text { magnification. Equal tear layer thickness in } \\
\text { central zone, increased tear layer thickness at } \\
\text { edge, no points of pressure or air bubbles }\end{array}$ \\
\hline \multicolumn{3}{|l|}{ Over-refraction: } \\
\hline Spherical only & Within + / - $0.25 \mathrm{D}$ & Highest plus power with subjective refraction \\
\hline Cylindrical only & Within + / - $0.50 \mathrm{D}$ & Balanced using cross cylinder method \\
\hline Residual toricity & $\leq$ Cross cyl 0.25 & Measured using cross cylinder method \\
\hline Visual acuity & Within one line & $\begin{array}{l}\text { Snellen visual acuity compared to best } \\
\text { corrected spectacle vision }\end{array}$ \\
\hline Wearing time & Acceptable to patient & $\begin{array}{l}\text { Recorded as full time if minimal } 8 \text { hours of } \\
\text { daily wear at least } 6 \text { days a week. Recorded } \\
\text { as limited wear if less than full time but } \\
\text { acceptable to patient. }\end{array}$ \\
\hline Slit lamp examination & CCLRU ${ }^{20}$ standards & $\begin{array}{l}\text { Standards for ocular tissue changes as } \\
\text { specified for daily wear }\end{array}$ \\
\hline Subjective findings & $\mathrm{CCLRU}^{20}$ standards & Checklist questionnaire for comfort and vision \\
\hline
\end{tabular}


were ordered accordingly and checked on production accuracy. Lenses with radii differences of greater than $0.02 \mathrm{~mm}$, dimension differences of greater than $0.05 \mathrm{~mm}$, or power differences of greater than $0.12 \mathrm{D}$ from the ordered value were rejected. At the dispensing visit the lenses were placed on the eyes and the fit was evaluated separately by two masked investigators. This evaluation included movement on the blink, lens centration, evaluation of static and dynamic fluorescein pattern and slitlamp evaluation of tear layer and edge clearance. Criteria for a good fit were as given in Table 4. If there was agreement between the investigators on the presence of a good fit, the lenses were dispensed. If there was agreement on a possible improvement of the fit and the amount of change, new lenses were ordered and the evaluation procedure repeated. If there was disagreement between investigators, a third investigator was asked to play an impartial role.

All patients received instruction on the assigned care system and all followed the same scheme of adaptation to the lenses ( 4 hours on the first day with increases of 1 hour each subsequent day). Follow-up visits were scheduled at day 10 , week 6 , months 3 and 6 , and finally at year 1 following dispensing. At follow-up visits the anterior segment of the eye was monitored, wearing time registered, the fit evaluated, and the condition of the lens scored according to the Rudko classification (Table 5). Also, the BOZR of the lenses was measured on each follow-up using a radiuscope. Successful wear was defined as the ability to wear the lenses for 8 hours a day, 6 days a week.

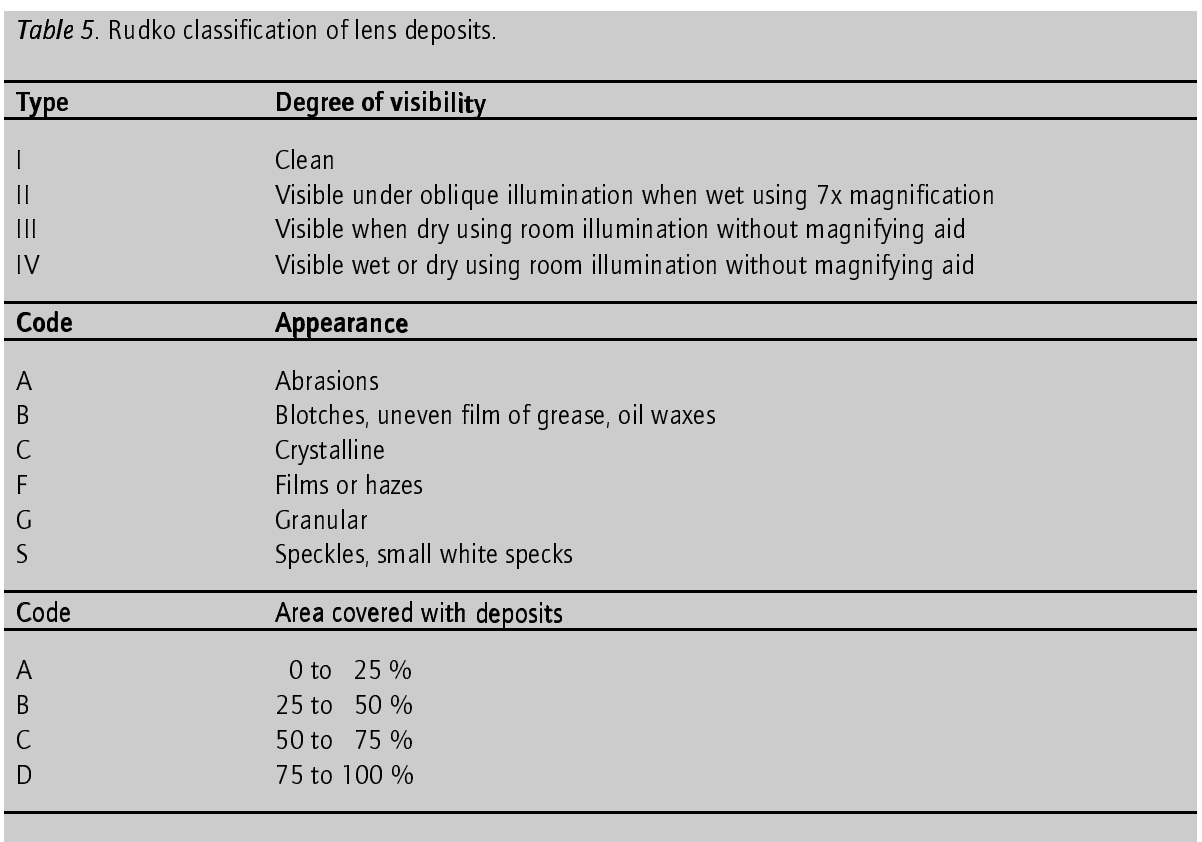




\subsubsection{Results}

Prediction of BOZR: For 61 patients (122 eyes) the two selection methods could be analyzed for their ability to predict BOZR in accordance with the clinical evaluation. For the 58 eyes in which the flat-K method was used, the computed BOZR was in accordance with the clinical evaluation (successful fit) in 48 eyes $(83 \%)$ while in ten eyes $(17 \%)$ the predicted fit was too flat. In none of the eyes was the predicted fit too steep. The corneal topometry method showed in 48 of 64 eyes $(75 \%)$ a prediction in accordance with the clinical evaluation; in six eyes (9\%) the prediction was too flat and in ten eyes (16\%) the fit was too steep (Fig. 3). The better prediction of the BOZR with the flat-K method was not significant $(P>0.01)$. To further analyze the predictions of both systems we used the computed average flattening (E-values) and the differences in peripheral flattening per semi-meridian per eye and labeled the good and fault predictions. Figure 4 shows clearly that the reason for fault (too flat) prediction with the flat-K method must be found in a significantly $(P<0.01)$ lower E-value, i.e., less flattening than average. Since the topometrical method inherently compensates for peripheral flattening, it is not necessary to look at average flattening in this group. Figure 5 presents the fault prediction in relation to the maximum difference between the semi-meridians of the same eye. It shows that there were significantly $(P<0.001)$ more fault predictions in eyes that had large differences in flattening between the semi-meridians.

Prediction of Diameter: Diameter prediction via the selection guide method as analyzed on 58 eyes showed good accordance with clinical evaluation (successful fit) in 52 eyes (90\%); in 4 eyes $(7 \%)$ the predicted lenses were too small; in two eyes $(3 \%)$ too large. Of the 64 eyes that were selected for prediction of TD via supraversion, $46(72 \%)$ were in accordance with clinical evaluation (successful fit), ten (16\%) were too small, and eight (12\%) too large.

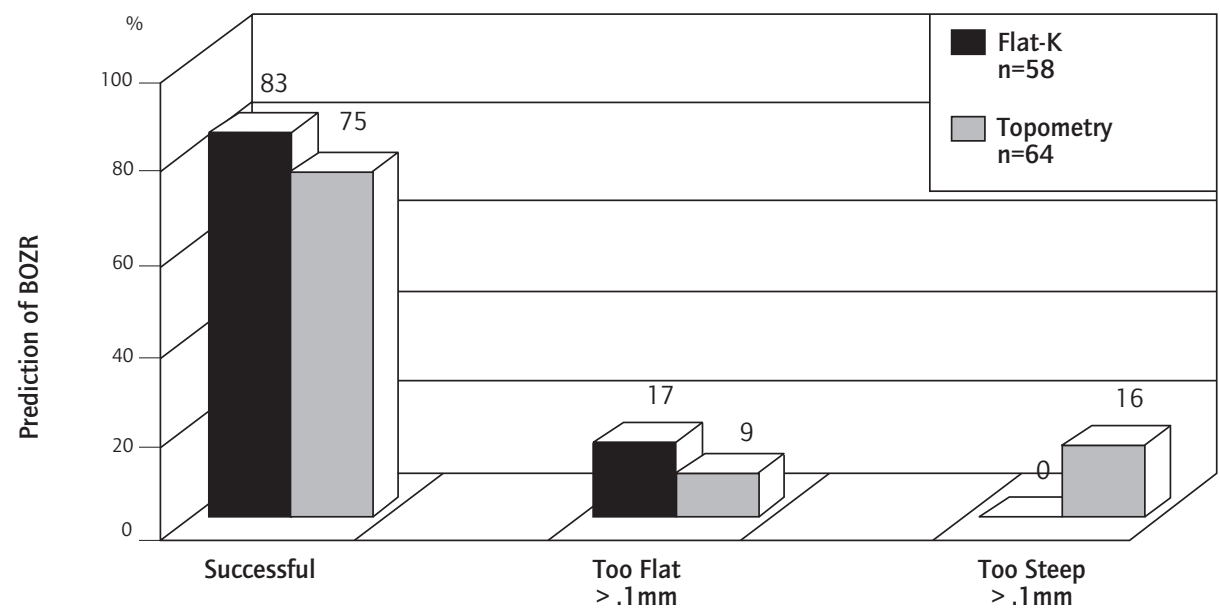

Figure 3. Prediction of BOZR using flat-K method or topometry. The flat-K method predicted BOZR successfully in $83 \%$ of the eyes. This is better (but not significantly better [P>0.01]), than the $75 \%$ successful predictions of BOZR by the topometric method. 


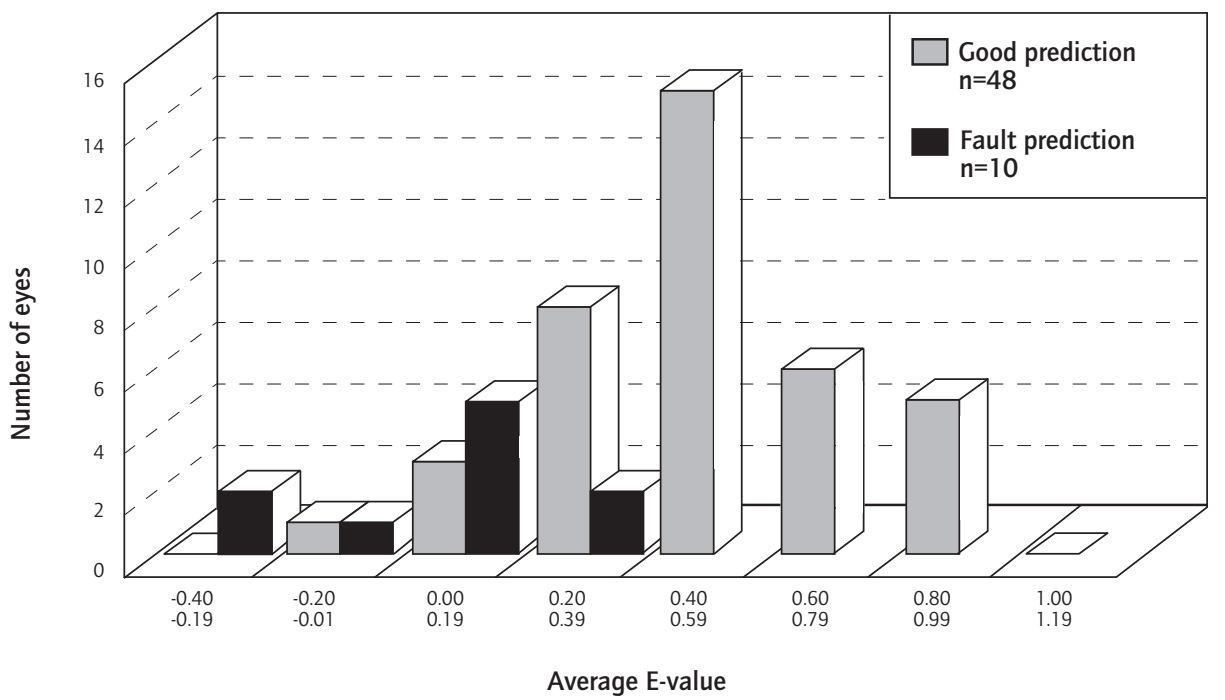

Figure 4. Distribution of average E-values for good and fault predictions of BOZR by the flat-K method. Fault predictions show significantly $(P<0.01)$ lower average E-values.

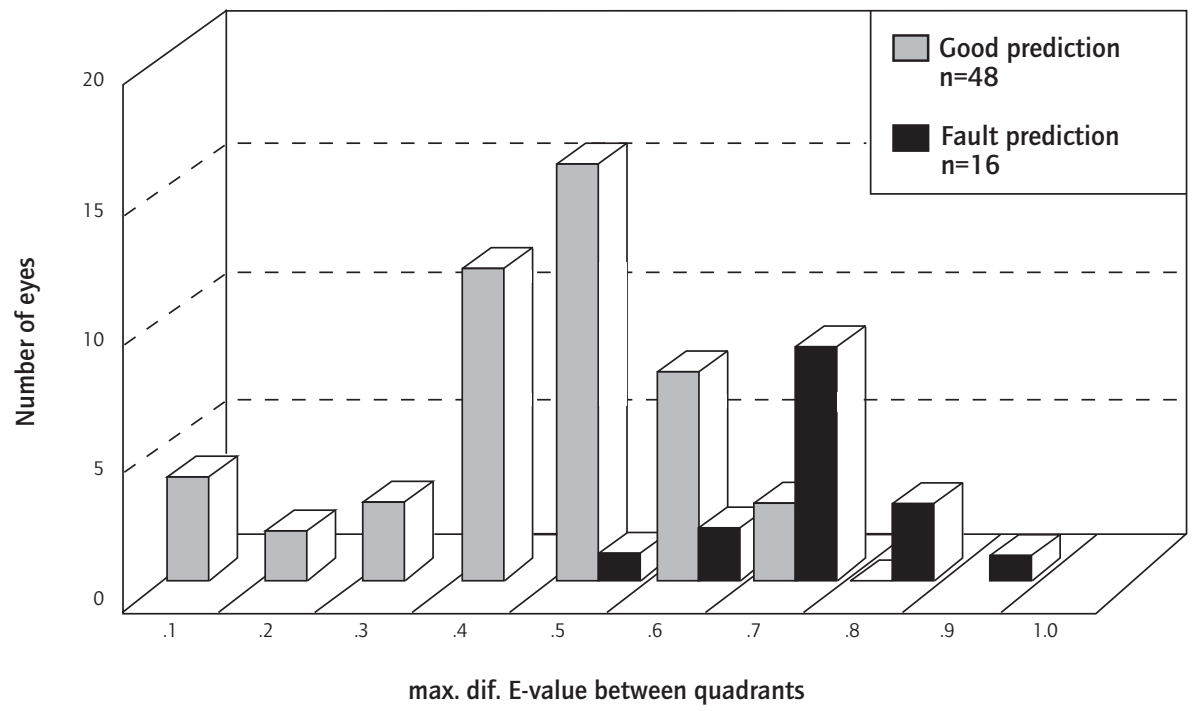

Figure 5. Distribution of maximal difference in E-value between semi-meridians of the same eye in relation to good and fault prediction of BOZR by the topometric method. Fault predictions show significantly $(\mathrm{P}<0.001)$ more differences in E-value between quadrants in the same eye. 
A summary of these results is given in Figure 6. Statistical analysis (chi square test, $P<0.01$ ) confirmed the hypothesis that the selection guide method is a better predictor of lens diameter than the supraversion method.

Traditional vs One-Step chemical cleaning: Table 6 summarises the results of observations on the lens and eye condition over the total follow-up period of 1 year. Both cleaning methods performed almost equally well. There is a tendency with the one-step chemical cleaner for higher deposit formation and mild injection, whereas the traditional cleaner system tended to give more 3-9 o'clock staining. However, none of these differences were statistically significant $(P>0.05)$. We found no BOZR changes greater than $0.10 \mathrm{~mm}$. With these results the hypothesis that the one-step solution would do worse in keeping the lenses in good condition was rejected. Also the hypothesis that traditional cleaning system would lead to more deformation of the lenses was rejected.

Final success: Of the 66 patients entering the study, five (7.5\%) discontinued lens wear within 6 months and five (7.5\%) wore their lenses on only a limited basis after 1 year. The reasons for discontinuation and limited wear are given in Table 7. Successful wear was found in 56 patients $(85 \%)$.

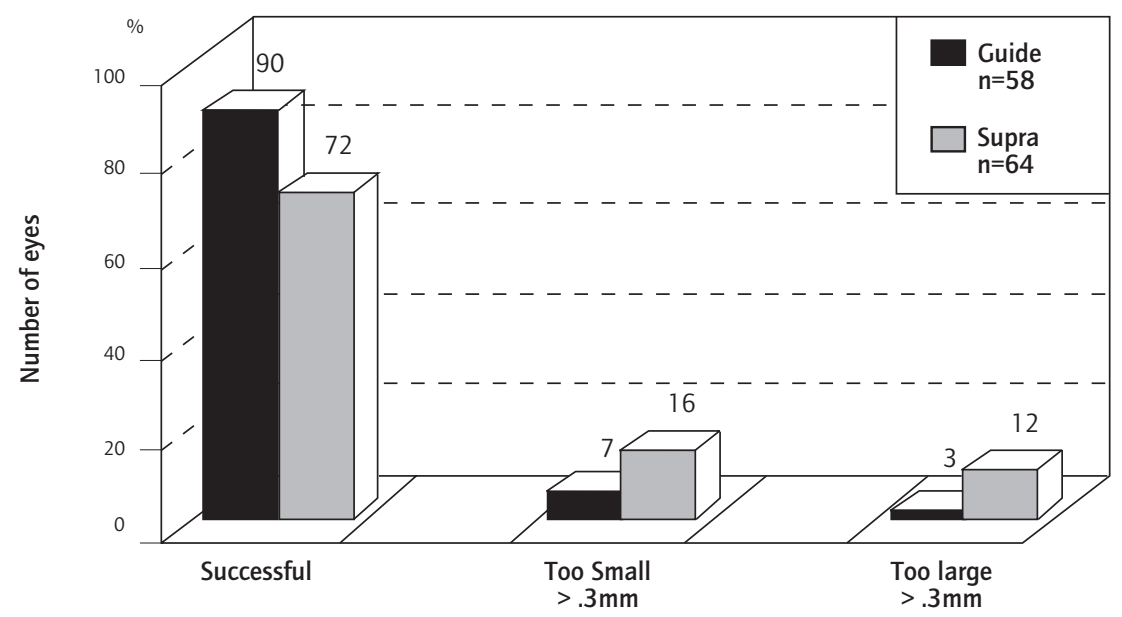

Figure 6. Prediction of diameter by the new guide and supraversion method. The guide method showed to predict diameter significantly better $(\mathrm{P}<0.01)$ than the method of supraversion.

\subsubsection{Discussion}

Prediction of BOZR: Our results with respect to prediction of BOZR did not significantly favor of the topometric method. The fact that the flat-K method showed a tendency to predict a flatter BOZR could be explained by the nature of the flat $\mathrm{K}$ method. Classic multicurve lens designs tend to have a steeper fit. The topometric method resulted in both too flat and too steep fits. One would suspect that more information on corneal shape would 
Table 6. Comparison between the care systems: one-step chemical cleaner vs traditional care system with separate surfactant cleaner. Differences are not statistically different $(P>0.05)$. Note figures can add to greater than $100 \%$ since several signs and symptoms may be present in one eye.

\begin{tabular}{|c|c|c|c|c|}
\hline \multirow{2}{*}{$\begin{array}{l}\text { Signs and symptoms } \\
\text { None }\end{array}$} & \multicolumn{2}{|c|}{$\begin{array}{l}\text { One step cleaner } \\
n=68(100 \%)\end{array}$} & \multicolumn{2}{|c|}{$\begin{array}{l}\text { Surfactant cleaner } \\
\mathrm{n}=54(100 \%)\end{array}$} \\
\hline & 54 & $(79.4 \%)$ & 43 & (79.6\%) \\
\hline Mild injection & 6 & $(8.8 \%)$ & \multicolumn{2}{|c|}{--} \\
\hline 3-9 o'clock staining & \multicolumn{2}{|c|}{-- } & 3 & $(5.5 \%)$ \\
\hline Debris & 5 & $(7.3 \%)$ & 3 & ( $5.5 \%)$ \\
\hline Change in $\mathrm{K}>0.10 \mathrm{~mm}$ & 2 & $(2.9 \%)$ & 2 & ( $3.7 \%)$ \\
\hline Scratches & 4 & $(7.4 \%)$ & 4 & ( $7.4 \%)$ \\
\hline Deposits & 8 & $(11.8 \%)$ & 2 & ( $3.7 \%)$ \\
\hline Broken & 1 & $(1.5 \%)$ & 1 & $(1.8 \%)$ \\
\hline Change in BOZR $>0.10 \mathrm{~mm}$ & \multicolumn{2}{|c|}{--} & \multicolumn{2}{|c|}{--} \\
\hline
\end{tabular}

Table 7. Fitting spheric-aspheric high Dk Exel RGP contact lenses: successes and failures $(N=66)$.

\begin{tabular}{llllll}
\hline Total Patients & Discontinued & Limited wear & Successful \\
\hline Beginning of the study & Limbal injection & 1 & $3-9$ o'clock staining & 1 & Wearing the lenses \\
& Comfort & 2 & Dryness & 3 & 8 hours per day, six days \\
& GPC & 1 & GPC & 1 & per week \\
& Retinal pathology & 1 & & \\
\hline 66 patients (100 \%) & 5 patients (7.5\%) & 5 patients $(7.5 \%)$ & 56 patients (85\%) \\
\hline
\end{tabular}

give a better prediction rate. However, some inherent limitations of the topometric method are known ${ }^{15}$ and topography does not always fit theoretical models. ${ }^{16}$ Since the starting point for lens calculation in the topometric method is the alignment on central-K, the fault predictions are probably related to peripheral flattening. Studies on keratoconus indicate that differences in flattening between the semi-meridians in an eye can reveal large differences in desired lens parameters. 5 From our calculations it seems that this may also hold true for normal eyes. It should be pointed out that this can happen in both flatter or steeper peripheral shapes, explaining why the topographic method revealed both too steep and too flat fits. Compensation for this effect should incorporate a weighting averaging system. Applying this modified computation method on our data increased the success rate of predicting BOZR with the topometric method to $92 \%$. Further research on a new group of patients is needed to prove the validity of this calculation method.

Diameter selection: The results of diameter selection favored the new method. It could be shown from the present study that the relation between corneal radius and corneal diameter forms a good basis for computation of the lens diameter. Although we used larger diameters for high Dk RGP lenses, our findings are comparable to those found by Boyd ${ }^{17}$ in using small, thin polymethylmethacrylate (PMMA) lenses.

The use of other parameters as extra factors for diameter selection in our method is in accor- 
dance with a study by Postma and coworkers. ${ }^{15}$ They concluded that the use of more parameters than aperture size alone might be helpful to increase initial comfort. This concurs with our results using the diameter method showing adequate diameter selection in $90 \%$ of the cases.

Care systems: Our results showed no statistical difference between the one-step chemical and traditional system of care for high Dk RGP contact lenses. Taking into account that in daily practice many patients would refrain from using more complex care systems, the one-step system is very attractive from a standpoint of patient compliance. However, one should realise that a one-step system does not obviate the need for extra cleaning. Good follow-up remains important. ${ }^{18,19}$ Finally, it appears that digital cleaning with a surfactant in the traditional care system has no significant effect on lens stability. This is in accordance with earlier research of our group ${ }^{13}$ involving other materials. It seems that the traditional view that high Dk RGP materials are unstable is no longer valid. The reason for instability of high $\mathrm{Dk}$ RGP contact lenses as described in the past ${ }^{12}$ may not always be related to properties of the material but to "mistreatment" of these materials by traditional manufacturing methods in combination with heavy polishing. The present study leads us to conclude that modern computer numeric controlled (CNC) manufacturing contributes significantly to lens stability.

Final success: The final success rate (85\%) with the Exel high Dk RGP lenses in this study is comparable to the success rates reported in other studies using similar high Dk RGP lenses. ${ }^{1,16}$ In a study by Jones and coworkers ${ }^{1}$ using Persecon 92E high Dk RGP lenses produced from Fluoroperm 90 material, the success rate after 6 months follow-up was exactly the same. Postma and coworkers ${ }^{16}$ using Fluoroperm 30 material found a success rate of $92 \%$ with a trial fit method and $86 \%$ with a nomogram fit (after 3 months follow-up). De Brabander and coworkers ${ }^{13}$ found a success rate of $77 \%$ using RXD and Equa 2 material (after 1 year follow-up).

In this study, high Dk RGP contact lenses produced with modern materials and manufacturing techniques could be fit with a success rate of $85 \%$. Failures included a $7.5 \%$ discontinuation rate due to comfort, lens intolerance and lack of motivation. Also considered failures were the additional $7.5 \%$ of patients who had reduced wearing rates due to dry eye symptoms.

Computation of the BOZR, using topometry incorporating averaging of corneal flattening did not give better results than simple computation based on central flat- $\mathrm{K}$ and corneal astigmatism. With a different calculation system based on weighted average corneal flattening, topometry seems to be able to achieve much better $(92 \%)$ results in predicting BOZR. Selection of diameter could be done very efficiently ( $90 \%$ success rate) using the newly developed scheme for Exel high Dk RGP contact lenses.

In summary, even with the advanced systems of lens computation used in this study it is not yet possible to achieve a success rate of more that $92 \%$ success in predicting the BOZR and $90 \%$ in predicting the diameter of rigid contact lenses. These results are beneath those required by common quality standards of practice. Thus we cannot recommend a direct ordering system of rigid contact lenses using our method.

The one-step solution (Unicare Green) performed equally well in keeping the lenses in good 
condition compared with a more complicated system containing specially formulated solutions. The use of a surfactant cleaner in the traditional care system did not result in lens deformation. A way to increase compliance and quality of lens care could be to start with the one-step solution and in those cases where extra cleaning is required, use a surfactant cleaner as needed.

\section{Acknowledgement}

We thank ICN/Oculenti for their support and Kam Cheng, MD for his help in computing E-values.

With this publication we honor our dearest friend and initiator of this study, Dr. Jan H.C. Kok who died much too early on November 21, 1998.

\section{References}

1. Jones L, Jones D. A new high Dk elliptical lens. Optician 1992;Jan 10:16-23.

2. Atkinson T. The ideal RGP back surface design for daily wear. Optician 1990;March 2:34-36.

3. Wilms K, Rabbetts R. Practical concepts of corneal topometry. Optician 1977;Sept 16:7-13.

4. Wilms K. Ueberlegungen zur Topometrie von Cornea und Kontaktlinsen. Neues Optiker Journal 1980;15:569-571.

5. Kok J, Wagemans M, Rosenbrand R, Mil van C. Computer assistance in keratoconus lens design. CLAO J 1990;16:262-265.

6. Kok JH, Smulders F, van Mil C. Fitting of aspheric high gas-permeable rigid contact lenses to scarred corneas. Am J Ophthalmol 1991;112:191-194.

7. Mandell R. Methods of direct ordering. Contact Lens Practice. Ed. 2, Springfield, Charles C Thomas, 1977.

8. Kok J, Rosenbrand R. Indications and contra-indications for extended-wear of soft and rigid contact lenses. Contactologia 1987;9:180-183.

9. Walker J. High Dk RGPs are the lenses of first choice. Optician 1992;Febr 7:18-25.

10. Kok J, Vreugdenhil W. Extended-wear high oxygen-permeable Boston Equa and Fluoroperm contact lenses. Contactologia 1990;12:163-167.

11. Schwartz C. Contact lenses in the 1990s: fitting for lifestyles. Contact Lens Forum 1990;Jan:17-29.

12. Walker J. Comparing the base curve stability of various contact lens materials. Contact Lens Forum 1989;March:59-69.

13. Brabander de J, Brinkman CJJ, Kok JHC, van Mil C. Low or high Dk? Spherical or aspherical rigid gas permeable lenses? The gap between theory and practice. J BCLA 1992;15:159-162.

14. Contact lens usage in the Netherlands. Consumer survey, Intomart, 1982, Hilversum, The Netherlands.

15. Fedtke D. Die Sagittalradienvermessung der Hornhaut - Kritische Betrachtung einer populaer gewordenen Anpaszphilosophie. Deutscher Optiker Zeitung 1984;4:103-113.

16. Postma JT, Postma AM, Schnider CM. A comparison between nomogram vs. trial fitting of rigid gas permeable contact lenses. J Am Optom Assoc 1993; 64:258-263.

17. Boyd HH. Evaluation of 1,000 patients wearing small thin contact lenses. Nippon Kontakuto Renzu Gakkai Kaiin Dayori 1969;11:13-5. 
18. Turner FD, Gower LA, Stein JM, Sager DP, Amin D. Compliance and contact lens care: a new assessment method. Optom Vis Sci 1993;70:998-1004.

19. Gower LA, Stein JM, Turner FD. Compliance: a comparison of three lens care systems. Optom Vis Sci 1994;71:629-634.

20. Terry RL, Schnider CM, Holden BA, Cornish R, Grant T, Sweeney D, La Hood D, Back A. CCLRU standards for success of daily and extended wear contact lenses. Optom Vis Sci 1993;70:234-243. 
Chapter 4

Soft Contact Lenses, Special Optical Designs 


\subsection{Introduction to this chapter}

In this chapter we present some of our work with special optically designed SCLs.

In section 4.2 the topic is the correction of astigmatism with so-called toric SCLs. Fitting toric SCLs has been complicated by physiological, technical and methodological problems. Low water content materials in combination with thick lens designs, necessary for lens stabilisation, can lead to low oxygenation of the eye. ${ }^{1}$ Poor reproducibility in manufacturing toric SCLs, especially for cylinder power and axis, has been reported as a major cause of discrepancy between expected and actual behaviour of the lens on the eye., ${ }^{2,3}$ In combination with poor reproducibility in manufacturing, the traditional methodology of fitting via trial lenses has led to confusing and disappointing results. 4,5 Modern Computer Numeric Controlled (CNC) lathe techniques make it possible to produce thinner lens designs in high water content materials with good reproducibility. A custom, rather than trial lens, way of prescribing toric soft contact lenses looks very promising. ${ }^{6}$ In a multi- centre study we evaluated the clinical results of a system to prescribe custom-made toric SCLs with high water content material and thin design.

Section 4.3 is dedicated to the possibilities of correcting presbyopia by CLs in general and specifically by varifocal SCLs. For many years CL science has been trying to find a solution to correct presbyopia by means of multifocal CLs. ${ }^{7-9}$ This has proved to be a very difficult task. We studied the literature to make an inventory of factors in the contact lens - eye presbyopia complex. From this we designed a clinical study to investigate the influence of lens design, addition, pupil size and lens position on far and near visual acuity for prototyped varifocal SCLs.

In section 4.4, modern optical designs of the anterior surface of SCLs are the topic of study. The principle behind this type of SCLs is, in addition to correcting for spherical/cylindrical ametropia, also to correct higher-order aberrations of the eye. ${ }^{10-12}$ This is particularly important in cases of high ametropia and deformations like in keratoconus. With specially designed and controlled SCLs we studied the effects of spherical and aspherical anterior lens surfaces on contrast sensitivity of the eye in high ametropia. Also the feasibility of SCLs suitable to optically correct higher-order aberrations in keratoconus was investigated (4.5).

\section{References}

1. Hallak J, Cohen H. Localized edema with soft toric contact lenses. J Am Optom Assoc 1985;56:920-1.

2. Remba MJ. Part II. Clinical evaluation of toric hydrophilic contact lenses. J Am Optom Assoc 1981;52:211-21.

3. Payor RE, Robirds SR, Zhang X, Schwallie JD. Soft toric lens power accuracy and reproducibility. CLAO J 1995;21:163-8.

4. Castellano CF, Myers RI, Becherer PD, Walter DE. Rotational characteristics and stability of soft toric lenses. J Am Optom Assoc 1990;61:167-70.

5. Silbert J, Ghormley NR, Hankin B, Rigel L, Barron C. An evaluation of empirically fitting a posterior toric hydrogel contact lens. J Am Optom Assoc 1992;63:170-5. 
6. Hall DK, Ward JA, Edmondson W. Spectacles and custom toric hydrogel contact lenses: a comparison of vision. J Am Optom Assoc 1994;65:783-7.

7. Feinbloom W. Segmented optics in scleral lenses to correct presbyopia. United States Patent, 1938.

8. De Carle JT. Bifocal and multifocal contact lenses. Contact lenses: a textbook for practitioner and student, Ed. 3, Edited by Stone. London, Butterworths, 1989

9. Back A. Presbyopes and contact lenses is there hope? Optician 1991;july 5:16-9.

10. Westheimer G. Aberrations of contact lenses. Am J Optom Arch Am Acad Optom 1961;38;8:445-8.

11. Cox I. Theoretical calculation of the longitudinal spherical aberration of rigid and soft contact lenses. Optom Vis Sci 1990;67:277-82.

12. Cox I, Holden BA. Soft contact lens-induced longitudinal spherical aberration and its effect on contrast sensitivity. Optom Vis Sci 1990;67:679-83. 


\subsection{Soft Toric Contact Lenses}

\section{Clinical evaluation of a Custom-made Toric Soft Lens System}

J. de Brabander ${ }^{1,6}$, C.J.J. Brinkman 2,6 , R.M.M.A. Nuijts' ${ }^{1}$ C. van Mili3,6, T. Sax ${ }^{4}$ and E. Brookman 5 .

From:

1 Eye Research Institute Maastricht, Department of Ophthalmology, University Maastricht, the Netherlands.

2 Hospital De Heel, Zaandam, the Netherlands.

3 van Mil Contact Lenses, Almere, the Netherlands.

4 Academic Hospital, Oculenti practice, Utrecht, the Netherlands.

5 Eurolens Contact Lens Practise, Venlo and Helmond, the Netherlands.

6 Academic Medical Center at the University of Amsterdam, Department of Ophthalmology, Oculenti practice, Amsterdam, the Netherlands.

Contact Lens \& Anterior Eye 2000;23;1:22-8. 
Abstract

Purpose. The fitting of toric soft contact lenses is often complicated by interference between the toric optics and the geometry needed to stabilise the lens on the eye. The use of a spherical trial lens to determine the parameters of a toric soft contact lens can lead to unintended positioning and movement of the lens ordered. The purpose of this study was to evaluate clinically the success rate of an empirical method of prescribing custom-made thin, high water content toric soft contact lenses.

Method. The design of the lenses was such that optical correction and stabilisation were separated. The lenses had a back toric surface limited to the central optical zone and base down prism stabilisation in the periphery. The empirical system used corneal radii, spectacle refraction and horizontal visible iris diameter as lens design parameters.

Results. This multi-centre trial, comprised of 105 patients (193 eyes), revealed an overall success rate of $86 \%$. The total number of lenses ordered to achieve success was 1.46 per eye in new patients and 1.69 per eye in patients that were failures with other toric soft lenses. In $14 \%$ of the cases success could not be achieved despite ordering several lenses (up to 4 lenses/eye). Many of these patients (60\%) were also failures from other toric soft lenses.

Conclusion. It is concluded that in new patients successful toric soft contact lens fitting can be achieved with an empirically based method. When refitting former toric soft lens failures, we recommend discontinuation after two further unsuccessful fittings.

\section{Key words:}

Astigmatism, toric soft contact lenses, empirical fitting, custom-made contact lenses. 


\subsubsection{Introduction}

Unlike rigid contact lenses, a spherical soft lens does not correct corneal astigmatism due to the absence of a toric liquid lens. Ever since the introduction of spherical soft lenses it has been recognised that this optical different results in reduced visual acuity (VA) in cases where the spectacle cylinders are greater than $0.75 \mathrm{D} .{ }^{1}$ To overcome this problem the soft lens needs to incorporate a toric design which will stabilise at the appropriate cylinder axis. The special geometry needed to both correct the astigmatism and stabilise the lens on the eye requires that a whole range of considerations be taken into account. ${ }^{2-4}$ The classical concept of stabilising toric contact lenses on the eye is the incorporation of a so-called "prism ballast". This term suggests that stabilisation is solely a function of gravity but it has been shown by Hanks ${ }^{5}$ that this is of little significance in stabilisation, the dominant force being lid squeezing. With toric soft lenses, it is more appropriate to refer to (dynamic) stabilisation zone(s) as suggested by Fanti. ${ }^{6}$ The role of lid squeezing in relation to the elasticity and the thickness profile of the lens is also known to be an important factor in stabilising toric contact lenses ${ }^{7}$, especially if correction of moderate to high oblique astigmatism is needed. The major problems in toric soft lens fitting can be categorised as:

1. Stabilisation of the lens on the eye to maintain the appropriate cylinder axis.

2. Low oxygenation of the cornea especially in low water content lenses with a thick lens design necessary for stabilisation.

3. Complicated fitting procedure using diagnostic trial lenses.

4. Discrepancy between behaviour of trial lens and the lens ordered.

5. Difficulties at follow-up visits in establishing the subjective refraction (especially with "off axis" lenses) and conflict between VA results in comparison with reported visual dissatisfaction (fluctuations in vision) by the patient.

6. Poor reproducibility of re-ordered lenses.

Recent developments in high water content materials and manufacturing techniques have led to a variety of new designs of toric soft contact lenses. Lenses can now be made thinner due to specially constructed stabilisation zones ${ }^{8}$ and the choice between front and back toric designs makes the fitting more flexible. ${ }^{2,9}$ The use of materials free of hydroxyethyl methacrylate (HEMA), especially in toric soft contact lens fitting, has been reported as minimising the development of giant papillary conjunctivitis. ${ }^{10,11}$ However, since it is clear that frequent replacement is the most significant factor in preventing soft contact lens complications ${ }^{12}$, standard toric contact lens systems have been developed by various manufacturers. With these systems frequent replacement even for toric soft contact lenses has become economically feasible. Despite all these advances it seems that, as with to the fitting of aspheric rigid gas permeable (RGP) contact lenses ${ }^{13}$, practitioners hesitate unnecessarily in prescribing toric soft contact lenses. ${ }^{14,15}$ Silbert et al. ${ }^{16}$ suggested that this reluctance may be a result of the existing perception that prescribing toric soft lenses is complex, unpredictable and time consuming. In their study of a simplified empirical fitting method, solely based on keratometry and refractive error, they found a success-rate of $75 \%$ when "success" was defined as VA with contact lenses equal to, or better, than that with spectacles. When they used the criterion of VA with contact lens- 
es within one line of best corrected spectacle VA, the success-rate was $95 \%$. When patients' satisfaction with VA was used as a criterion, the success rate became $84 \%$. Their conclusion was that empirical fitting provided a simple method of achieving successful toric soft lens fitting for the majority $(75 \%)$ of patients.

In studies on empirical fitting, refractive information solely, or in combination with keratometry, is used to select the most appropriate lens parameters available in the standard design and range of back vertex powers offered by the manufacturer. Previous studies of empirical fitting have not considered the selection of total diameter despite its importance as a factor in the position, movement and rotation of a soft contact lens. ${ }^{17}$ Hence it is worthwhile investigating the value of this parameter in an empirical fitting system. Furthermore, in standard systems, cylinder axis can usually only be chosen as nearest to the available $5^{\circ}$ within a range of $+/$ $20^{\circ}$ with respect to the horizontal and to the vertical. One might question whether more precise specification of cylinder axis, especially in oblique astigmatism, would improve success rate. However, inclusion of these extra parameters necessitates the use of a custom-made lens, rather than selection from a range of standard parameters.

In diagnostic fitting more information can be derived from assessment of the trial lens on the eye. Also, as suggested by Silbert et al. ${ }^{16}$ it would be interesting to explore further the idea of using the empirically ordered lens as a diagnostic one in those case in which it was unsuccessful.

Modern manufacturing techniques have made it possible to offer an empirical fitting system that uses custom-made toric soft contact lenses at reasonable costs. One of these, the Igel $\mathrm{Rx}$ toric soft lens, was chosen for this investigation in an endeavour to establish the following:

1. Success rate of the first ordered lens.

2. Increase in success rate with subsequently ordered lenses.

3. The number of lenses ordered in both successful and unsuccessful cases.

4. The reasons for failure.

\subsubsection{Material and Methods}

A total of 105 patients (193 eyes), 37 male and 68 female, participated in a multi-centre study. Inclusion criteria were age between 20 and 60 years, a spectacle cylinder of $>0.75 \mathrm{D}$ and no history of eye pathology. New wearers, as well as failures with other soft toric lenses, participated after obtaining their oral informed consent. The initial visit included a routine examination for fitting of contact lenses and the measurements necessary for the ordering of the lenses. All lenses used in this study were produced by Igel International (Igel, Leighton Buzzard, UK). The Igel Rx Toric utilises a thin lens design with a back toric surface limited to the central optical zone (Fig. 1). The periphery is spherical for adequate scleral alignment. The stabilisation of the lens is achieved by means of a base down prism that is present only in the peripheral part of the lens to prevent binocular vertical imbalance. In this study, we used a 58\% water content material for all lenses. Table 1 summarises the lens design and material properties. This lens employs a simple ordering system that is related to a computerised manufacturing process. All lenses were ordered according to the manufacturer's instructions. Ordering data included 


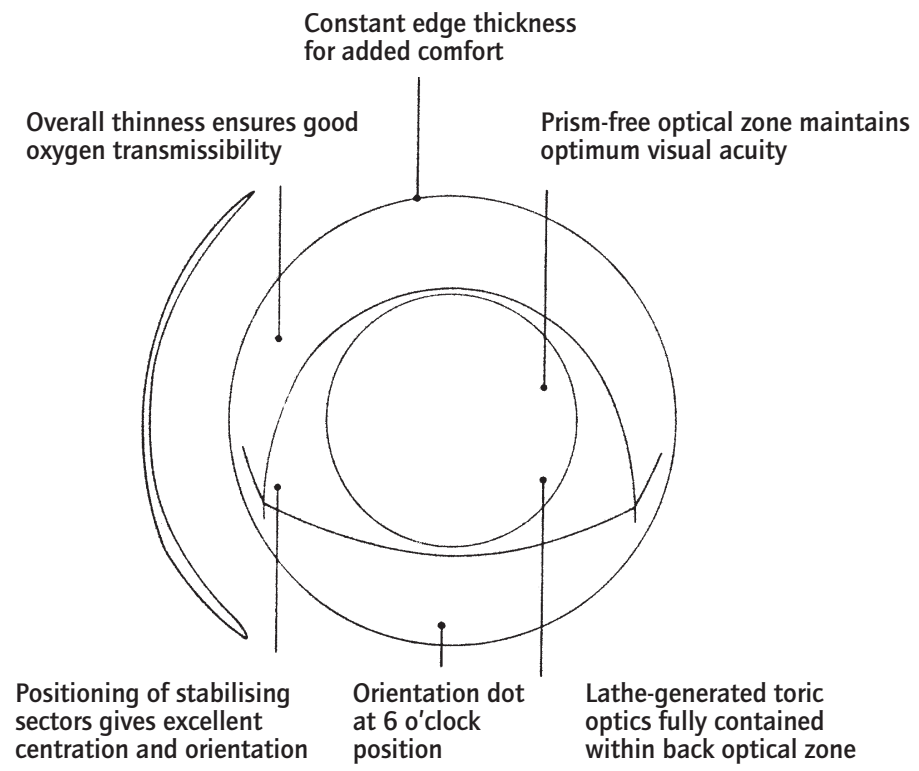

Figure 1. Design of the Igel Rx Toric soft contact lens.

flattest and steepest keratometry readings with meridians, spectacle spherical and cylindrical refraction with axis, vertex distance and horizontal visible iris diameter (HVID). From these data the manufacturer's computer determined the input for the production of the lens on a computer numeric controlled (CNC) lathe.

On the dispensing visit the fitting was evaluated after $30 \mathrm{~min}$, to allow settlement. The lenses were assessed for fit in terms of movement, centration and visual acuity using the criteria as outlined in Table 2. The patient was then instructed on lens handling and care. The care system issued to all patients was Unicare Blue (ICN, Pharmaceuticals, Zoetermeer, the Netherlands). The wearing schedule was $4 \mathrm{~h}$ on the first day with an additional $1 \mathrm{~h}$ each consecutive day.

Table 1. Material properties and parameters of the Igel Rx Toric soft lens.

Material

Water content

Back optic zone radius

Back optic zone diameter

Total diameter

Geometrical centre thickness

Spherical power

Cylindrical power

Cylinder axis
Filcon 4a (after BS 7208:Part 2: 1991)

$58 \%$

Determined by the manufacturer on the basis of keratometry

$8.25 \mathrm{~mm}$ (at $-3.00 \mathrm{D})$

Determined by the manufacturer on the basis of HVID

$0.10 \mathrm{~mm}$ (at $-3.00 \mathrm{D})$

To any specification

-0.25 to $-11.00 \mathrm{D}(0.25 \mathrm{D}$ intervals)

To any specification ( $1^{\circ}$ intervals) 


\begin{tabular}{|c|c|c|}
\hline Feature & Criterion & Description \\
\hline Movement & $0.5-1.0 \mathrm{~mm}$ & On blinking \\
\hline Centration & $>0.5 \mathrm{~mm}$ & $\begin{array}{l}\text { Extension of lens edge beyond the limbus in all directions } \\
\text { including movement on the blink }\end{array}$ \\
\hline Stabilisation & Within $10^{\circ}$ & $\begin{array}{l}\text { Average position of orientation dot from vertical plane } \\
\text { using five recordings after forced blinking }\end{array}$ \\
\hline Rotation & Within $10^{\circ}$ & $\begin{array}{l}\text { Differences between min. and max. angle position of } \\
\text { orientation dot using five recordings after forced blinking }\end{array}$ \\
\hline \multicolumn{3}{|l|}{ Over-refraction: } \\
\hline Spherical only & Within +/- $0.25 \mathrm{D}$ & Highest plus power subjective refraction \\
\hline Cylindrical only & Within +/- $0.50 \mathrm{D}$ & Balanced using cross cylinder method \\
\hline Residual toricity & $\leq$ Cross cyl 0.25 & Measured using cross cylinder method \\
\hline Visual acuity & Within one line & $\begin{array}{l}\text { Snellen visual acuity compared to best corrected } \\
\text { spectacle vision }\end{array}$ \\
\hline Wearing time & Acceptable to patient & $\begin{array}{l}\text { Recorded as full time if minimal } 8 \text { h of daily wear, } \\
\text { minimal } 6 \text { day's a week. Recorded as limited wear if lower } \\
\text { but acceptable to patient. }\end{array}$ \\
\hline Slit lamp examination & CCLRU standards ${ }^{20}$ & $\begin{array}{l}\text { Standards for ocular tissue changes as specified for daily } \\
\text { wear }\end{array}$ \\
\hline Subjective findings & CCLRU standards ${ }^{20}$ & Check-list questionnaire for comfort and vision \\
\hline
\end{tabular}

Follow-up visits were at 6 weeks, 3 months and 6 months after dispensing. At each follow-up visit lens movement, centration, stabilisation, rotation, over-refraction, distance visual acuity, wearing time, slit-lamp evaluation and subjective findings were recorded. A detailed description of these items and the criteria for a successful fit are shown in Table 2. If either symptoms or findings did not meet one or more of the specified criteria with the first lens ordered the investigators were allowed to a diagnostic fitting procedure using their observations to order a new lens. This procedure was if necessary repeated for up to four lenses per eye.

\subsubsection{Results}

From the 105 patients (193 eyes) who entered this study, 15 patients (14\%), including 28 eyes, did not complete the study up to the last follow-up visit at 6 months. Reasons for failure are given in Table 3. Of the 15 failure cases, $9(60 \%)$ were former failures of other toric soft lenses. The remaining 90 successful patients $(86 \%, 165$ eyes) can be divided in a group that was able to wear the lenses full-time (78 patients, $74 \%$ ) and a group (12 patients, $11 \%$ ) that was satisfied with the lenses but used them with limited wearing time.

Distribution of the refractive spherical equivalent and cylinder power for the 90 successful patients (165 eyes) is given in Figs. 2 and 3, respectively. Mean refractive spherical equivalent power \pm standard deviation $(\mathrm{SD})$ was $-3.07 \mathrm{D} \pm 5.72$ with a range of $-15.00 \mathrm{D}$ to $+15.00 \mathrm{D}$. Mean minus cylinder power $\pm \mathrm{SD}$ was $-2.23 \mathrm{D} \pm 1.19$ ranging from $-1.00 \mathrm{D}$ to $-7.50 \mathrm{D}$. In 


\begin{tabular}{|c|c|c|c|c|}
\hline & Male & Female & Total & $\%$ \\
\hline Entered the study & 37 & 68 & 105 & 100 \\
\hline \multicolumn{5}{|l|}{ Failure } \\
\hline Intolerance & 3 & 1 & 4 & (3.8) \\
\hline Dryness symptoms & 0 & 2 & 2 & (1.9) \\
\hline Visual problems & 3 & 2 & 6 & (5.7) \\
\hline Rotation & 1 & 1 & 2 & (1.9) \\
\hline No follow up & 1 & 1 & 2 & (1.9) \\
\hline Total discontinued & 8 & 7 & 15 & 14.3 \\
\hline \multicolumn{5}{|l|}{ Successful } \\
\hline Full-time wear & 21 & 57 & 78 & (74.3) \\
\hline Limited wear & 8 & 4 & 12 & (11.4) \\
\hline Total successful & 29 & 61 & 90 & 85.7 \\
\hline
\end{tabular}

our patient group, $68 \%$ showed astigmatism with-the-rule with minus cylinder axis between $160^{\circ}$ to $20^{\circ}, 12.5 \%$ against-the-rule (axis $70^{\circ}$ to $110^{\circ}$ ) and $19.5 \%$ an oblique astigmatism (axis $20^{\circ}$ to $70^{\circ}$ or $110^{\circ}$ to $160^{\circ}$ ). Mean keratometer readings $\pm S D$ were $7.85 \mathrm{~mm} \pm 0.24$ with a range of 7.18 to $8.62 \mathrm{~mm}$. Horizontal visible iris diameter as recorded in intervals of $0.5 \mathrm{~mm}$ averaged $11.5 \mathrm{~mm}$, ranging from 10.5 to $12.5 \mathrm{~mm}$.

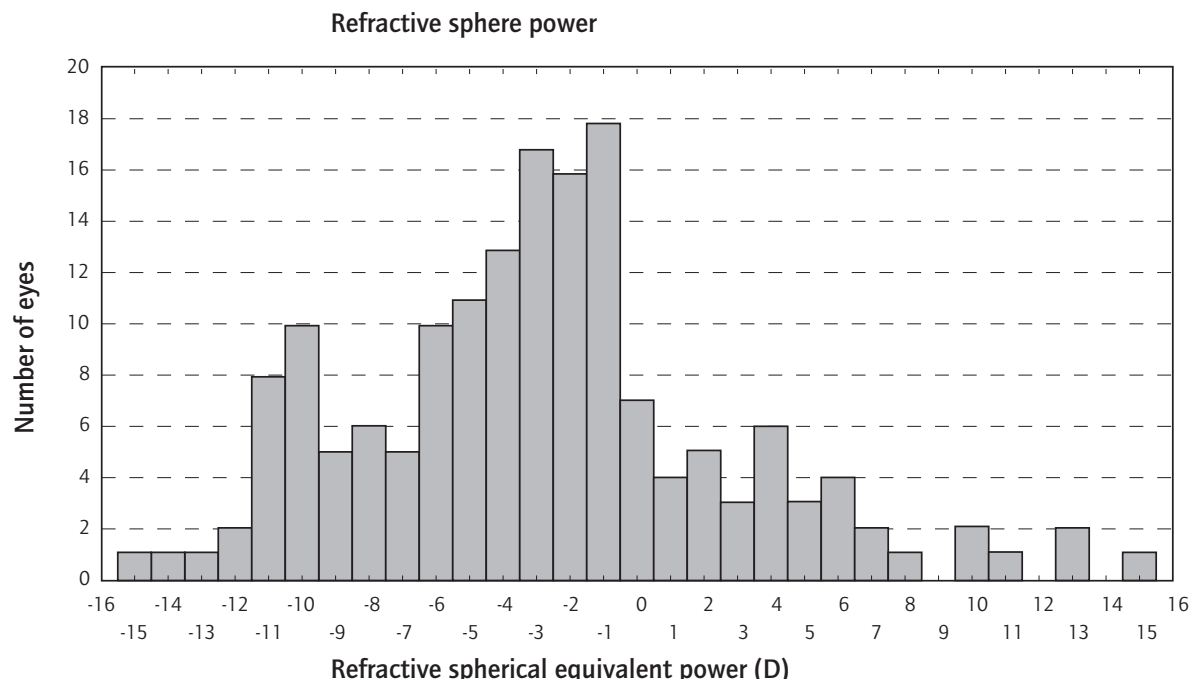

Figure 2. Distribution of refractive spherical equivalent power $(n=165)$. 


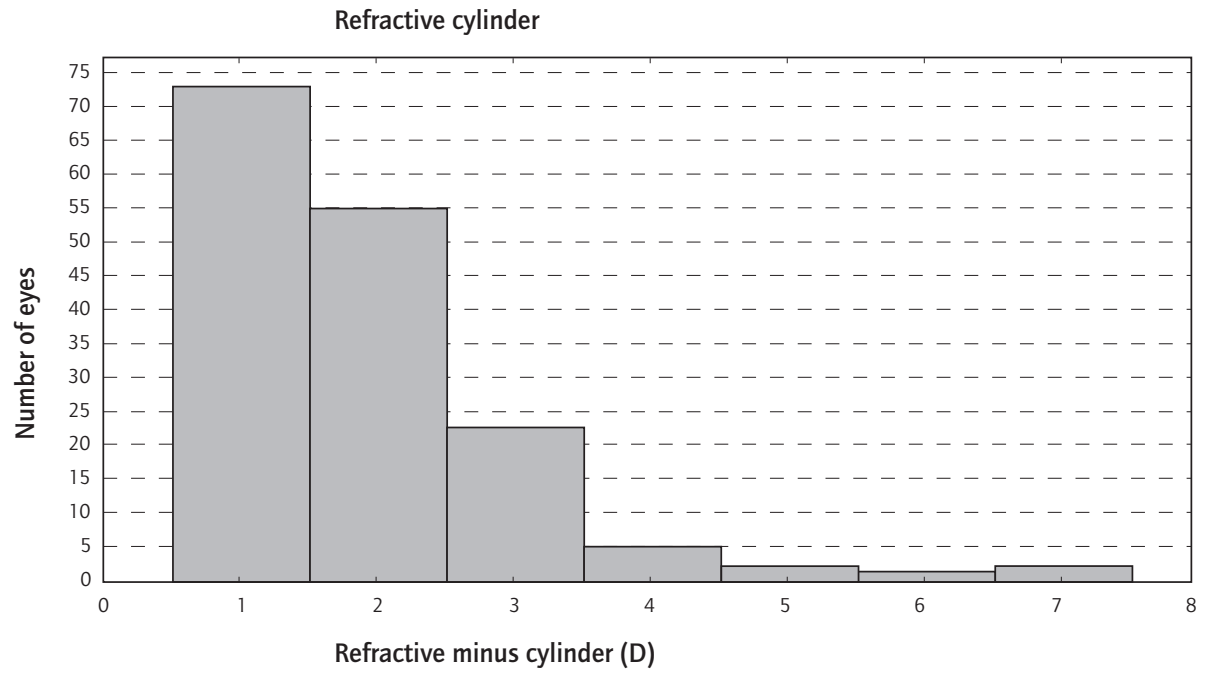

Figure 3. Distribution of refractive cylindrical power $(n=165)$.

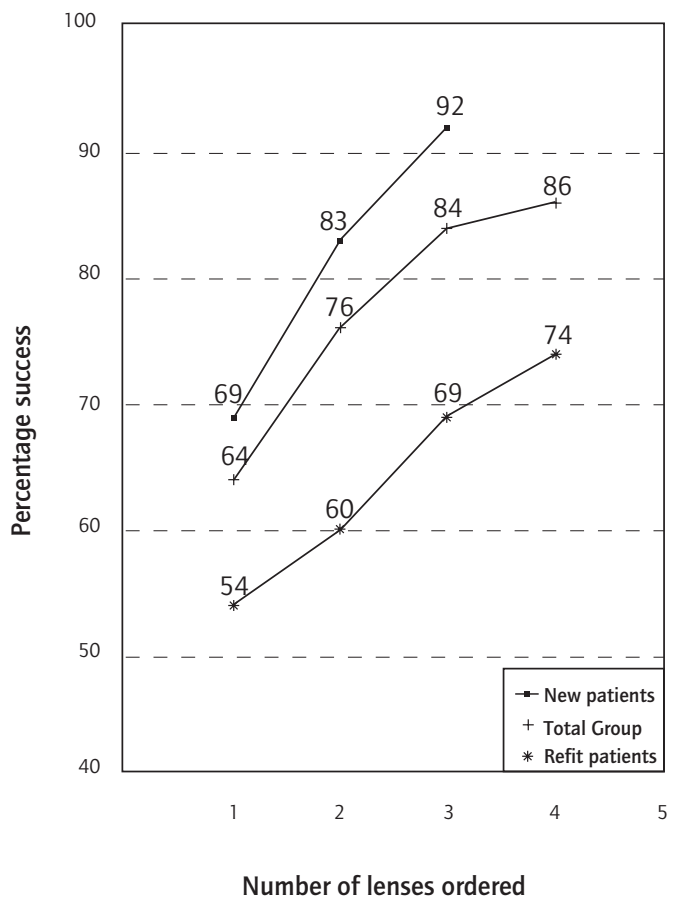

Figure 4. Increase in success rate for new wearers, re-fits and the total group in relation to the number of lenses ordered. 
In Figure 4, the success rate is given with the number of lenses needed to achieve it for the total group, for the group of new wearers and for the group of re-fits. The difference in success rate between new wearers of $92 \%$ and re-fits of $74 \%$ is statistically significant (Chi-square, $p<0.1$ ). A further breakdown in groups of with-the-rule, against-the-rule and oblique astigmatism revealed that type of astigmatism was not a statistically significant factor for success or failure in the group of new wearers. In the group of re-fitted patients, only the presence of oblique astigmatism was found to be statistically significant in failure cases. It can be seen from the slope of the lines in Fig. 4 that in new wearers the increase in success rate per next ordered lens is higher than with the re-fitted patients.

We needed an average of 1.53 lenses per eye to accomplish success in the whole study, 1.46 lenses per eye to fit new patients and 1.69 lenses per eye in re-fitted patients. In the group of successful patients, we used 1.38 lenses per eye. This is significantly lower (Mann-Whitney test, one-sided $p<0.05$ ) than in the group of failures where we used 2.4 lenses per eye.

In Table 4, the performance of the first and second lens ordered is related to the fitting, total diameter, stabilisation, rotation and power. Results showed $6.5 \%$ too steep and $1.5 \%$ too flat

\begin{tabular}{|c|c|c|}
\hline & First lens & Second lens \\
\hline \multicolumn{3}{|l|}{ Fitting } \\
\hline Good & 92.0 & 98.0 \\
\hline Steep & 6.5 & 1.0 \\
\hline Flat & 1.5 & 1.0 \\
\hline \multicolumn{3}{|l|}{ Total Diameter } \\
\hline Good & 89.5 & 100.0 \\
\hline Too small & 8.0 & -- \\
\hline Too large & 2.5 & -- \\
\hline \multicolumn{3}{|c|}{ Stabilisation (degrees) } \\
\hline $0-5$ & 71.0 & 84.5 \\
\hline $5-10$ & 24.5 & 12.5 \\
\hline $10-20$ & 3.5 & 2.0 \\
\hline 20 or more & 1.0 & 1.0 \\
\hline \multicolumn{3}{|l|}{ Rotation (degrees) } \\
\hline $0-5$ & 78.5 & 86.0 \\
\hline $5-10$ & 16.0 & 11.5 \\
\hline $10-20$ & 2.0 & 1.0 \\
\hline 20 or more & 3.5 & 1.5 \\
\hline \multicolumn{3}{|l|}{ Over-refraction } \\
\hline Sph. within $+/-0.25$ & 91.0 & 99.0 \\
\hline Cyl. within +/- 0.50 & 89.0 & 95.0 \\
\hline Residual toricity & 9.0 & 2.5 \\
\hline
\end{tabular}


fits on the first lens order. Both figures were reduced to $1.0 \%$ on the second lens order. The total diameter for the first lens was too small in $8 \%$ and too large in $2.5 \%$. Both were corrected completely by ordering a second lens. In $95.5 \%$ of the eyes, stabilisation (see Table 2) was within 10 degrees and could be improved to $97 \%$ on the second ordered lens. For rotation (see Table 2), these figures were $94.5 \%$ and $97.5 \%$, respectively. On the first lens ordered, spherical and cylindrical power were within the criterion for success in $91 \%$ and $89 \%$, respectively. In $9 \%$ of the eyes, the first lens ordered showed a residual toricity, illustrating an over-refraction with a cross cylinder power of $0.5 \mathrm{D}$ or more.

Visual acuity results, including data from failures, are presented in Figure 5 as the difference in measured Snellen VA (decimal notation) between spectacle and toric soft lens correction. The "within one line difference" in VA with the toric lens and the best spectacle correction applied to $89 \%$ of the eyes. In $51 \%$ of the eyes, VA with the toric soft lenses was equal or better than with best spectacle correction. In $80 \%$ of cases the patients were satisfied with their VA while wearing the toric soft lenses.

During the period of follow-up no statistically significant changes in lens performance were found (Student's $t$-test, $\mathrm{p}>0.05$ ). In $15.5 \%$ of the cases, patients needed additional cleaning (mechanical and/or protein tablets) of the lenses to remove formed deposits. No adverse reaction was seen in the eyes of patients that completed the study. The patients that were discontinued did not show adverse physiological response on examination 2 weeks after discontinuation.

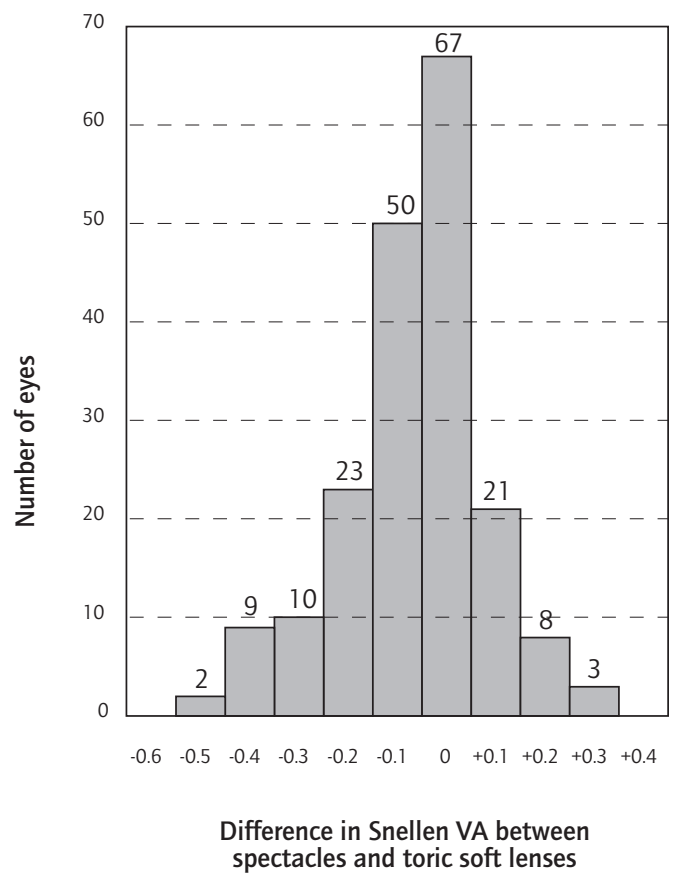

Figure 5. Difference in Snellen visual acuity VA (decimal notation) between spectacles and toric lenses $(n=193)$. 


\subsubsection{Discussion}

Compared to the relatively complicated diagnostic lens fitting procedures to prescribe toric soft contact lenses we found the empirical method as used in this study to be easy and straightforward. We estimate that the empirical method of fitting saves between 10 to $15 \mathrm{~min}$ at the initial visit.

Since most astigmatic patients are well aware of having 'difficult eyes' they were in general positively surprised by the ease of the fitting procedure. We noticed that a too optimistic way of explaining the system can be disappointing to both patient and practitioner in the event that the first lens does not work. This would be avoided by presenting the system realistically as ' $70 \%$ success on the first lens ordered'.

The data obtained from the present study made it possible to evaluate success rate in several ways. Therefore, especially in comparison with other studies, care must be taken to adequately specify definitions for success. Included in the present study were both new wearers, experienced wearers and former failures without restriction with respect to the type and the amount of their ametropia or astigmatism. Since this group represents the typical population practitioners are faced with in everyday practice it is legitimate to use the term overall success in evaluating the lens system. This figure gives a realistic view of what can be expected from the system in normal practice and is of special importance to the manufacturer in evaluating return and exchange policies, pricing etc.

We defined overall success rate as the percentage of patients that after the supply of one empirical or maximum of three consecutive diagnostic fittings met the specified criteria for fit and physiological response (Table 2), completed the 6 months follow-up and reported a wearing time and VA to their satisfaction. This definition resulted in a success rate of $86 \%$.

Success rates can also be defined more specifically for groups within the total. These figures can be used for practice management and information to an individual patient. If similar criteria as above are used for the sub-groups of new and re-fit patients the success rates are $92 \%$ and $74 \%$, respectively. In the same way the empirical fitting (first lens) shows success rates of 54\%, $64 \%$ and $69 \%$ for the groups of re-fits, total and new patients, respectively. The latter figure is slightly lower than found by Silbert et al. ${ }^{16}$ probably because they reported on a selected patient group (experienced wearers only, refractive power between plano and $-4.00 \mathrm{D}$, cylinder of $-1.25 \mathrm{D}$ or $-1.75 \mathrm{D}$ and only a 2 week follow-up).

Visual acuity measurements with the contact lenses in situ revealed $89 \%$ to be within one line and $51 \%$ equal or better than best spectacle correction, whereas $80 \%$ of the patients reported satisfactory vision. As also found by Silbert et al. ${ }^{16}$ visual acceptance varied. A relatively large group of patients accepted a loss of one line in VA, but a smaller group did not. However, the interpretation of these results should be made with caution because satisfactory vision is a variable with a high inter- and intra-individual variability. Visual demands, individual circumstances and fluctuations in VA are some of the many, yet difficult to quantify factors, involved in satisfactory vision.

Power of sphere and cylinder were easy to control in this study. Probably a good fit leads to easy control of power. This is in accordance with the observations of Gundel ${ }^{18}$ and Myers ${ }^{19}$ who found good comparison between spherical-cylindrical over refraction and performance. 
Using the empirically ordered lens (delivered within 2 weeks) as a diagnostic lens proved to be a successful tool in increasing success rate. This way of managing the patient with more difficult ocular parameters has the great advantage of saving chair time and positively influencing patient motivation.

The approach of ordering custom made toric soft contact lenses as used in this study worked well in the majority of the cases. Perhaps a slight improvement is possible to balance the tendency that we found that the first lens ordered, if not correct, showed more "too steep" than "too flat" fittings and more "too small" than "too large" total diameters. This could be done by using a large pool self-learning database system with feedback on fitting results.

In this study it was intended to assess the performance of the lenses over a period of 6 months without replacement. The results showed that during that period $15.5 \%$ of the patients needed some additional cleaning of the lenses to keep them functioning satisfactorily. With the criteria used, the conclusion must be that with the lens and care system used the other $84.5 \%$ of the patients were satisfactory corrected over a period of 6 months. Further follow-up is needed to find out what exact replacement frequency is most beneficial in terms of cost and performance.

With replacement of the lenses in the future, reproducibility can also be investigated. However, when ordering second lenses in this study, reproducibility seems to be good. In the majority of the cases the second ordered lenses performed as the practitioner intended.

\subsubsection{Conclusion and Recommendation}

The results show the Igel Rx toric lens system to be reasonably efficient and reliable. The first lens ordered using the empirical fitting system was successful in almost $70 \%$ of new cases. The use of the empirical fitted lens as a diagnostic lens was shown to be successful. With the ordering of a second or a third lens (average 1.46 lenses per eye) the success rate in new wearers was increased to $83 \%$ and $92 \%$, respectively.

The re-fitting of failures with other toric soft lenses should be attempted with caution. In cases where a second ordered lens is unsuccessful, discontinuation of further attempts would be advisable.

\section{Acknowledgement}

We thank ICN/Oculenti for their support in this study.

\section{References}

1. Holden, B. The principles and practice of correcting astigmatism with soft contact lenses. Australian Journal of Optometry 1975;58:279-299.

2. Geyer, H. Torische Weichlinsen und ihre Anpassung. Kontaktlinse 1993;27;9:11-25.

3. Davies, I. Soft toric contact lenses: a systematic approach to problem solving. Optician, 1989;197;5211: 16-18. 
4. Castellano, C.F., Myers, R.I., Becherer P.D. and Walter D.E. Rotational characteristics and stability of soft toric lenses. J Am Optom Assoc 1990;61:167-170.

5. Hanks, A.J. The watermelon seed principle. Contact Lens Forum 1983;8;3:31-33.

6. Fanti, P. The fitting of a soft toroidal contact lens. Optician 1975;169;4376:8-9,13,15-16.

7. Grant, R. Mechanics of toric soft lens stabilisation. Trans Br Contact Lens Assoc Conf 1986:44-47.

8. Parry-Wood, L. Practicle experience with a soft toric Rx contact lens. Optician 1990;200 ;5275:18-21.

9. Fraser, G. Practicle experience with a soft toric lens. Optician 1992;203 ;5345:19-22.

10. Ris, W. and Pautsch, J. CSI Clarity und CSI Clarity Toric: Eine Anpaszstudie. Deutscher Optiker Zeitung 1993;4:21-27.

11. Bucci, F.A. Jr., Tanner, J.B., Moody, K.J. and Myers, P.J. Clinical performance of the Optima toric contact lens versus the CSI toric contact lens. CLAO J 1997;23:43-48.

12. Solomon, O.D. et al.: A 3-year prospective study of the clinical performance of daily disposable contact lenses compared with frequent replacement and conventional daily wear contact lenses. CLAO J $1996 ; 22 ; 4: 250-257$.

13. Brabander de J., Brinkman, C.J.J., Kok, J.H.C. and Mil van C. Low or high Dk? Spherical or aspherical rigid gas permeable lenses? The gap between theory and practice. J Br Contact Lens Assoc 1992;15:159162.

14. Massare, J.S. Toric soft contact lens fitting considerations. J Ophthal Nurs Technol 1989;8:193-196.

15. Hall, D.K., Ward, J.A. and Edmondson, W. Spectacles and custom toric hydrogel contact lenses: a comparison of vision. J Am Optom Assoc 1994;65:783-787.

16. Silbert, J., Ghormley, N.R., Hankin, B., Rigel, L. and Barron, C. An evaluation of empirically fitting a posterior toric hydrogel contact lens. J Am Optom Assoc 1992:63:170-175.

17. Bibby, M.M. and Tomlinson, A. A model to explain the effect of soft toric lens design specifications on movement. Am J Optom Physiol Opt 1983;60;4:287-291.

18. Gundel, R.E. Determining appropriate cylinder correction with soft toric lenses. J Am Optom Assoc 1988;59:206-208.

19. Myers, R.I., Castellano, C., Becherer, P.D. and Walter, D.E. Lens rotation and spherocylindrical overrefraction as predictors for soft toric lens evaluation. Optom Vis Sci 1989;66:573-578.

20. Terry, R.L. et al. CCLRU standards for success of daily and extended wear contact lenses. Optom Vis Sci 1993;70:234-243. 


\subsection{Correction of Presbyopia with Contact Lenses}

Presbyopia and Contact Lenses: A Literature Review and a Clinical Study on Discriminant Factors in Varifocal Soft Contact Lens Fitting

J. de Brabander ${ }^{1,2}$, N. Chateau ${ }^{3}$, H. Molenaar ${ }^{4}$ and F. Bouchard ${ }^{3}$.

From:

1 Eye Research Institute Maastricht, Department of Ophthalmology, University Maastricht, the Netherlands.

2 Academic Medical Center at the University of Amsterdam, Department of Ophthalmology, Amsterdam, the Netherlands.

3 Ocular Sciences (formerly Essilor CL division), R\&D International, Créteil, France.

${ }^{4}$ Ocular Sciences (formerly Lunelle) s'-Hertogenbosch, the Netherlands.

Contactologia 2000;22:99-108. 
Abstract

Introduction. With a large group of the population entering the age of presbyopia there is a fast growing demand for contact lenses able to correct presbyopia. In spite of many years of experience the ideal optical correction of presbyopia by means of a contact lens seems as yet unavailable. Patient and lens selection criteria and successrates vary dramatically among reported studies. The most important reason for this is a relative lack of knowledge about the complicated changes induced in physiological optics and visual system function by having presbyopic contact lens correction in situ.

Purpose. The first purpose of the present study was to summarise the subject of contact lens correction in presbyopia and to report on possible discriminant factors. Second, using relevant discriminant factors, to design and execute a clinical study to investigate the success and failure with various prototyped varifocal soft contact lenses.

Methods. A literature search was done to describe modes of contact lens correction in presbyopia and relevant discriminant factors. The clinical study examined the effects of addition, pupil size, lens centration relative to the pupil and lens design on visual acuity with prototyped varifocal contact lenses. Ninety presbyopic subjects were selected to participate in a multi centre single-masked crossover trial. All subjects were fitted with several centre near aspheric varifocal lenses. The dioptric power mapping of the lenses was controlled by multiple-wave interferometry. Visual acuity with each lens prototype was measured for distance vision with horizontal gaze, and for near vision with downward gaze. In the same conditions, a specially designed infrared video-pupillometer was used to obtain repeated measurements of pupil diameter and lens position on every eye. The influences of the lens design and of the measured independent factors on visual acuity were tested through multi way analysis of covariance.

Results. A description of the various modes to correct presbyopia by means of a contact lens is given. We have identified seven different but interactive areas of the human visual and behaviour system that are involved in visual perception with a multifocal contact lens in situ. We have termed this the presbyopia complex. The statistical analysis of the clinical study exhibits significant effects of lens design, pupil size, addition, and lens centration on near visual acuity. A large decentration of the lens relative to the pupil, or a large pupil combined with a high addition, appears to be related with lower performance in near vision (VA near acceptance-rate $74 \%$ ). However, this relation was not sufficiently strong to allow a reliable prediction of individual patient's acceptance from initial measurements. In distance vision, only the influence of the lens design was found to be significant. Distance acceptance-rate was very high, 98\%. The overall success rate at 3 months follow-up was $68 \%$.

Conclusion. The correction of presbyopia by contact lenses is very complex, involving many conditions and factors. The design method that combines numerical simulations and clinical study of performance, as used with the prototyped lenses in this study, has shown to be a promising concept in designing varifocal soft contact lenses.

\section{Key words:}

Presbyopia, multifocal soft contact lenses, varifocal contact lenses, visual system-function. 


\subsubsection{Introduction and literature review}

\section{History of contact lenses for presbyopia}

The correction of presbyopia by means of a contact lens is not new. In most literature the patent of Feinbloom ${ }^{1}$ in 1938 is mentioned as the first written reference to correcting presbyopia by scleral contact lenses. With the exception of Williamson ${ }^{2}$, reporting on his work at the Contact Lens Society (UK) meeting in 1950, not much has been written on the clinical results with scleral lenses to correct presbyopia.

With the rigid corneal contact lens becoming more popular in the 1950's there was renewed attention to the design of a contact lens for presbyopes. Most textbooks on contact lenses show a variety of sometimes extraordinary inventions to correct presbyopia. ${ }^{3}$

Another milestone, resulting in many new designs during the 1980's, was the introduction of hydrogel contact lenses. Fortunately, many more of the experiments with these lenses have been documented in writing. Many papers have reviewed the state of the art at a given time. ${ }^{4-8}$ We refer to this literature as general introduction to the subject.

Although contact lenses to correct presbyopia have been available for some decades they have never become very popular as a so to speak "standard mode" in the average contact lens practice. It should be mentioned however that some practitioners, usually using their favourite lens design and fitting technique, have been successful (personal communication).

\section{Present status of contact lenses and presbyopia}

As from the mid 1980's there is a growing demand as well as an interesting marketing perspective to aim for correction of presbyopia by contact lenses. Farkas 9 identifies five demographic, economic and technological arguments for this. Firstly, the fast growing group of patients wearing contact lenses that became presbyopic created the initial demand. Secondly, the "baby boom" generation showed an interest in starting to wear contact lenses when they became presbyopic. The economic point often mentioned is that baby boomers are motivated to spend their money in a "look young" lifestyle. Thirdly, practitioners have become more interested because they are better able, contrary to mass merchandisers, to fulfil the needs in this specialised field of contact lens fitting. Fourthly, with technical improvements and the option of monovision success-rates are increasing. Fifthly, it is an opportunity for the older contact lens practitioner to fill in the gap of loosing the younger patients group and offer services to a "peer" group.

Market analysis shows a dramatic percentage of increase of presbyopes in the population. However, it also appears that the number of contact lenses ordered for presbyopic correction does vary in each country and in each practice, but in general is as low as $0.5-1.5 \%$ of all lenses ordered.6,8,10-12 Monovison is the most used technique.

In general, practitioners have found the fitting and prescribing of contact lenses for presbyopes a difficult and confusing task. Most practitioners report that they are not able to reproduce the good or even reasonable results from studies with various contact lenses for presbyopic correction. Major reasons for this discrepancy seem to be differences in patient selection criteria, lack of guidelines to problem solving, differences in the definition of success and motivation of both patient and practitioner. 
A first conclusion from history and the present state of correcting presbyopia by means of contact lenses is certainly that the ophthalmic industry is investing in product development, practitioners are becoming more and more motivated to fit these lenses but that there are still too many uncertainties to fully exploit the market perspectives. Economic, logistic or basic motivation factors do not seem to be the problem. It seems that the main reason for the discrepancy between "demand and delivery" may be found in visual functioning of the patient while wearing a contact lens correction for presbyopia.

For all of the reasons mentioned it is worthwhile to investigate discriminant factors in fitting contact lenses for presbyopes. The first purpose of the present study is therefore to identify factors that influence the performance of the eye-contact lens systems in use to correct presbyopia.

\section{Contact lens systems in use to correct presbyopia}

For many years bifocal rigid contact lenses of various designs have been used. With the rigid bifocal lens on the eye it is in principle, like in bifocal spectacles, possible to change from one power to the other by moving the eyes. This system is known as translating bifocals or alternating vision by segmented optics. A key problem is the stabilisation of the lens on the eye. This is somewhat in contradiction with the physiological aim of lens movement to allow proper tear circulation. Also, the thick lens design is not very attractive from a physiological point of view.

One has tried to overcome the critical positioning of the segmented contact lens by using lenses with concentric bifocal power parts. In these lenses however the size of the pupil may become a problem. ${ }^{4}$ Multifocal rigid contact lenses have been developed but were difficult to manufacture with reasonable reproducibility and were expensive. For all of these reasons rigid bifocal or rigid multifocal contact lenses have not become very popular. However, with the availability of modern submicron lathe techniques and high gas permeable contact lens materials some of the problems described above are obsolete or diminished. This has renewed attempts to use rigid contact lenses for correction of presbyopia. ${ }^{13}$

The system of monovision has become more popular to correct presbyopia by contact lenses. The idea is simply to correct one eye for distance vision and the other eye for near vision. The basic objection against this system is that binocular vision is disturbed intentionally. ${ }^{14}$ In early presbyopic patients, where the near addition is $<1.5 \mathrm{D}$, the disturbance of binocular vision is accepted by most patients. ${ }^{15,16}$ If the addition becomes higher patients feel uncomfortable and disturbed by "the other" blurred image in performing a task on a given distance. To overcome this type of problem, 'modified monovision' can be used in which a combination of presbyopic correction in one eye is combined with monofocal correction in the other, or two multifocal lenses are used with a shift of distance and near power between the two eyes.

In recent years soft contact lenses have been developed to correct presbyopia. A special problem in the use of soft contact lenses is the relation between physical power of the lens and its effect on physiological optics with the lens in situ. ${ }^{17}$ Soft contact lenses have the advantage over rigid lenses that they center well and show only little movement on the blink. Lens position is therefore no longer a problem. However, since the lens moves with the eye the 
alternated vision approach cannot be used. ${ }^{18}$ When two powers are provided (horizontal or annular) simultaneously over the pupil aperture the eye has independent from its position, the addition always available to correct presbyopia. This system is therefore called simultaneous vision with bifocal soft contact lenses. ${ }^{19}$ Most common is a power distribution consisting of a central circular area with one power and an annulus around it having the other power. The central part can have the power for near, such a lens is consequently called Centre Near (CN) or have the power for distance vision, Centre Distance (CD). Multizone refractive bifocals are also in use.

Two other designs in simultaneous vision are in use today. One is known as diffractive bifocals. Diffractive contact lenses have a circular diffraction grating cut on the posterior surface. ${ }^{20,21}$ The grating produces two images on the retina simultaneously. Because there are many periods of the grating within the pupil area the system does not depend on the pupil size as far as optical power is concerned. ${ }^{22}$ However, because two images are formed at the same time and use the same entrance pupil the image illuminance is reduced by $50 \%$. The same reduction may occur with refractive bifocals. An additional problem with diffractive lenses is that light may be diffracted not only in the 2 desired orders that will form near and far vision focuses, but also in spurious diffraction orders that may induce glare. A second additional problem is that most diffractive designs induce a high amount of chromatism. This creates a problem in contrast of the retinal image specially in reading under low illumination. ${ }^{23,24}$ Furthermore patients complain about haloes, flare and ghost images. ${ }^{8}$ Especially under low luminance conditions, like night driving, flare is reported to disturb vision.

Another design in simultaneous vision is the use of an aspheric lens surface that produces a range of powers over a specified area. These lenses are referred to as multifocal soft contact lenses. We prefer, because with the use of aspheric surfaces the power distribution is intentionally continuous, to use the term "varifocal" soft contact lenses.

This type of lens can be produced as $\mathrm{CN}$ or $\mathrm{CD}$. With these lenses a complete range of powers is available within the pupil aperture. Theoretically a patient should be able to see clear at all distances. This type of lens design therefore comes very near to the situation of a normal eye with accommodation. However, the varifocal area that is available depends in this system on the pupil size. The available light to form the image is spread over the entire range of images so illuminance of a certain image can become very low. Often patients complain about not being able to "grab" the image they need when performing a given task. This leads to a neuroconfusion and some patients are asking themselves "do I see it or not?" 25,26 Other patients are very happy with the system; they can see clear at all distances and call this the invention of the century.

Several clinical studies $27-30$ compared the results with refractive / diffractive bifocal lenses and monovision. More recently, the varifocal (multifocal aspheric) lens design has become subject of investigation. ${ }^{31-33}$

Table 1, derived from Back ${ }^{8}$ with some additional terms from us, summarises this review of contact lens systems in use to correct presbyopia and shows the differences in optical principle and the related problems we have discussed. 


\begin{tabular}{|c|c|c|c|}
\hline LENS TYPE & PRINCIPLE OF OPTICS & ADVANTAGES & PROBLEMS \\
\hline Monovision & Monofocal & $\begin{array}{l}\text { Perfect correction for } \\
\text { each given distance }\end{array}$ & $\begin{array}{l}\text { Binocular vision } \\
\text { Eye dominance } \\
\text { Higher additions }\end{array}$ \\
\hline Segmental bifocal & $\begin{array}{l}\text { Two distinct focal } \\
\text { lengths in the } \\
\text { segments }\end{array}$ & $\begin{array}{l}\text { Perfect correction in each } \\
\text { segment }\end{array}$ & $\begin{array}{l}\text { Position of lens } \\
\text { Eye geometry } \\
\text { Higher addition }\end{array}$ \\
\hline Concentric bifocal & $\begin{array}{l}\text { Two concentric distinct } \\
\text { focal lengths }\end{array}$ & $\begin{array}{l}\text { Two perfect corrections } \\
\text { in each ring segment }\end{array}$ & $\begin{array}{l}\text { Pupil size } \\
\text { Position of lens } \\
\text { Higher addition }\end{array}$ \\
\hline Diffractive systems & $\begin{array}{l}\text { Concentric bending of } \\
\text { ray's }\end{array}$ & $\begin{array}{l}\text { Two corrections using } \\
\text { the entire pupil area }\end{array}$ & $\begin{array}{l}\text { Neuro-confusion } \\
\text { Haloes and flare }\end{array}$ \\
\hline $\begin{array}{l}\text { Multifocal } \\
\text { Varifocal }\end{array}$ & Variable optical profile & $\begin{array}{l}\text { All corrective powers } \\
\text { available }\end{array}$ & $\begin{array}{l}\text { Distance vision } \\
\text { Near vision } \\
\text { Neuro-confusion } \\
\text { Distance vision } \\
\text { Near vision }\end{array}$ \\
\hline
\end{tabular}

\section{The presbyopia complex}

As a conclusion from the literature search we found that although the basic optical consequences of presbyopia are well understood, the influence it has on the patients visual system is very complex. Many factors, including environmental and psychological ones, determine the level of handicap that presbyopia means to the individual patient. If an optical correction, as a multifocal soft contact lens is added, the situation becomes very complicated and unpredictable. In fact it is the lack of a scientific model of the multifocal lens system in situ that leads to the situation in which practitioners are confused by the outcome of their intervention methods. We have composed seven different but interactive areas of the human visual and behavioural system that are involved in visual perception with a multifocal soft contact lens to correct presbyopia in situ. We call this the presbyopia complex.

\section{Physical optics.}

This area involves the physical optics and the design of the multifocal soft contact lens.

2. Dynamic physiological optics

Once the lens is placed on the eye physical optics are integrated into the physiological optic system of the eye. In soft contact lenses this means also that the physical optics are changed.

3. Transfer of light in the ocular media

Special attention should be given to the travel of light within the ocular media since the multifocal lens is a very special optical condition.

4. Retina level neuro-physiology

The new physiological system created by the dynamic interaction between the lens and the eye means a new way of input to the retinal neurological processes. 
5. Cortical level neurology

If input from the retinal processes gives unexpected cortical signals and responses, the neurological system can become confused.

6. Environmental and ergonomic factors

Modern life desires a high competence of people, in situations that are not always optimal from a visual science point of view. Task demands are an important factor.

7.Psychological factors

Confusion at the cortical level can in combination with environmental demands lead to behavioural disturbance. The motivation of the patient and even more the ability to accept some compromise are also important.

Looking at the areas involved one realises that gaining control over all the many factors, within the seven main parts of the presbyopia complex, is practically impossible. It, however, clearly shows why there is so much confusion and contradiction in this research area.

As a second purpose of this study we clinically investigated the contribution to success or failure of a selection of the mentioned factors using prototyped varifocal lenses in a range of various aspheric designs under controlled conditions. We have selected a limited set of factors that we were able to control and aimed to keep all others as far as possible constant.

\subsubsection{Materials and Methods}

\section{Discriminant factors}

A major problem found in multifocal lenses is image quality in central vision. We have chosen to give this problem priority. We hypothesised that important factors in image quality (visual acuity) for both far and near vision are lens optical profile, addition, pupil size and lens centration.

\section{Study design and fitting procedure}

The clinical part of this study was designed as a multi center single masked crossover trial. All subjects were successively fitted with a series (L and S, Fig. 1) of prototyped centre-near aspheric varifocal soft contact lenses. The design of the lenses was made unknown to the investigators. Fitting of the lenses involved slitlamp examination, keratometry and the normal procedures ${ }^{34}$ for evaluation of soft lens performance on the eye. Criteria for good fit were movement of 0.5 to $1.0 \mathrm{~mm}$ on the blink and centring such that the lens edge extended $>0.5 \mathrm{~mm}$ over the limbus in all directions including movement on the blink.

Visual acuity with each lens prototype was measured in distance vision with horizontal gaze, and in near vision with downward gaze. In the same conditions, a specially designed infrared video-pupillometer was used to obtain repeated measurements of pupil diameter and lens position on every eye. The accuracy of centration measurements was improved by chemically marking opaque rings in the periphery of the lenses. This set up and methodology is in detail described in part 5.3 of this thesis. 35 


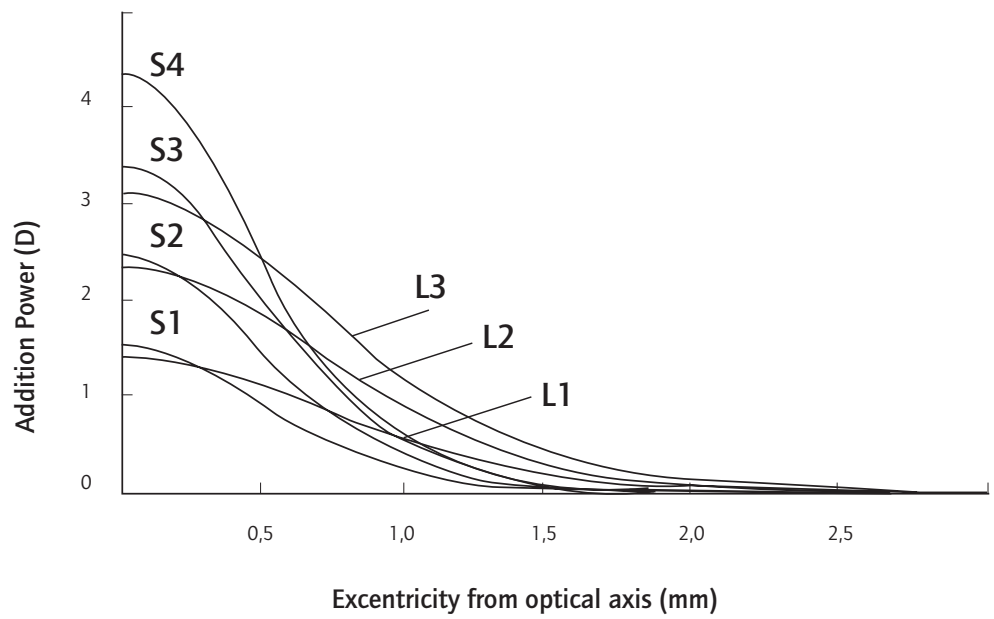

Figure 1. Power profiles of two varifocal lens design series. Group $L$ is designed with graduate power distribution featuring intermediate powers. Group $S$ aims to progressively reach near power at the centre of the lens.

\section{Subjects}

Subjects were recruited by written announcements placed at several locations within the premises of the University of Amsterdam, the Netherlands and at Essilor, Contact Lens Department, Creteil, France. At the initial visit 122 subjects underwent a complete ophthalmometric examination and were screened using the intake criteria as given in Table 2. A total of 99 subjects ( 44 males, 55 females) were selected to participate in this study after written informed consent as was approved by the Medical Ethical Committee of the University of Amsterdam. From these patients, 9 ( 6 males, 3 females) were excluded within three months after initial examination for reasons as difficulty in care and handling, motivation and moving. Complete data analysis was made on 90 patients ( 38 males, 52 females). The distributions in age, ametropia and addition are given in Figs. 2, 3, 4, respectively.

\section{Lenses}

The lenses used in this study had a centre near aspheric varifocal design. The lenses were produced on a modern CNC lathe (Euro Precision Technology, Almelo, the Netherlands) making it possible to control the power distribution (so-called optical profile) of the lens

\begin{tabular}{l} 
Table 2. Inclusion criteria \\
\hline Normal ocular condition \\
Normal binocular function \\
Age 40 to 70 years \\
Needed near addition $>0.50 \mathrm{D}$ \\
Refractive astigmatism $<1.00 \mathrm{D}$ \\
Sphere equivalent ametropia between $-8.00 \mathrm{D}$ and $+6.00 \mathrm{D}$ \\
\hline
\end{tabular}




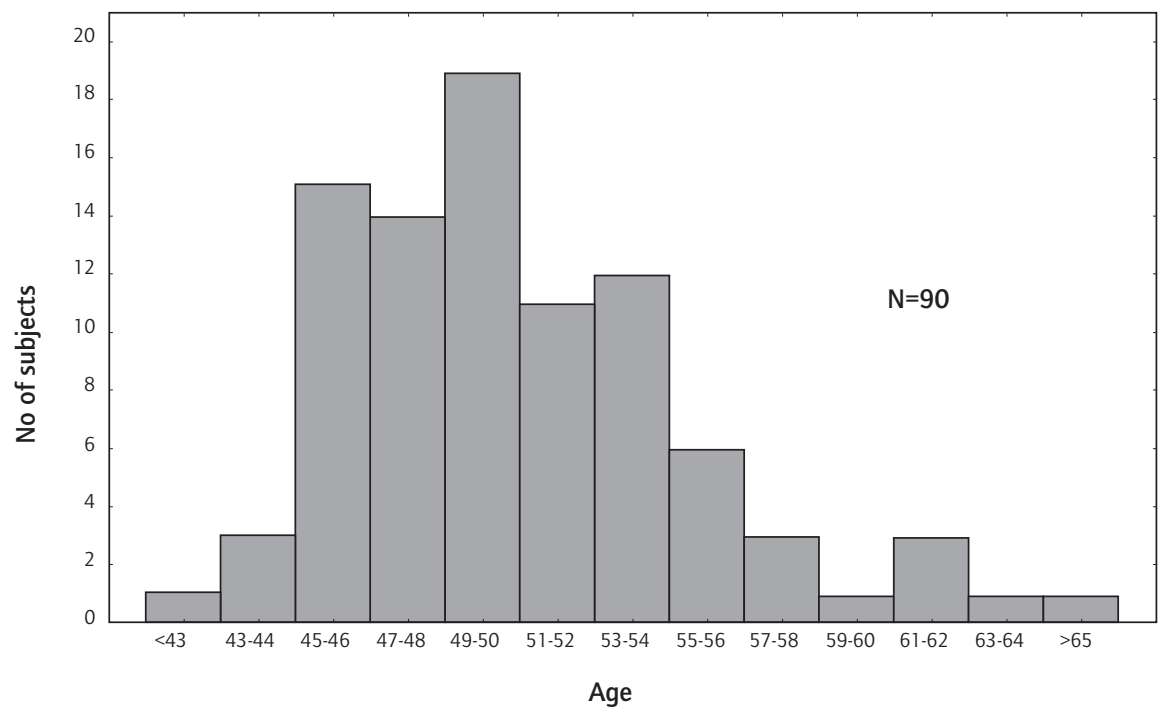

Figure 2. Age distribution of 90 subjects.

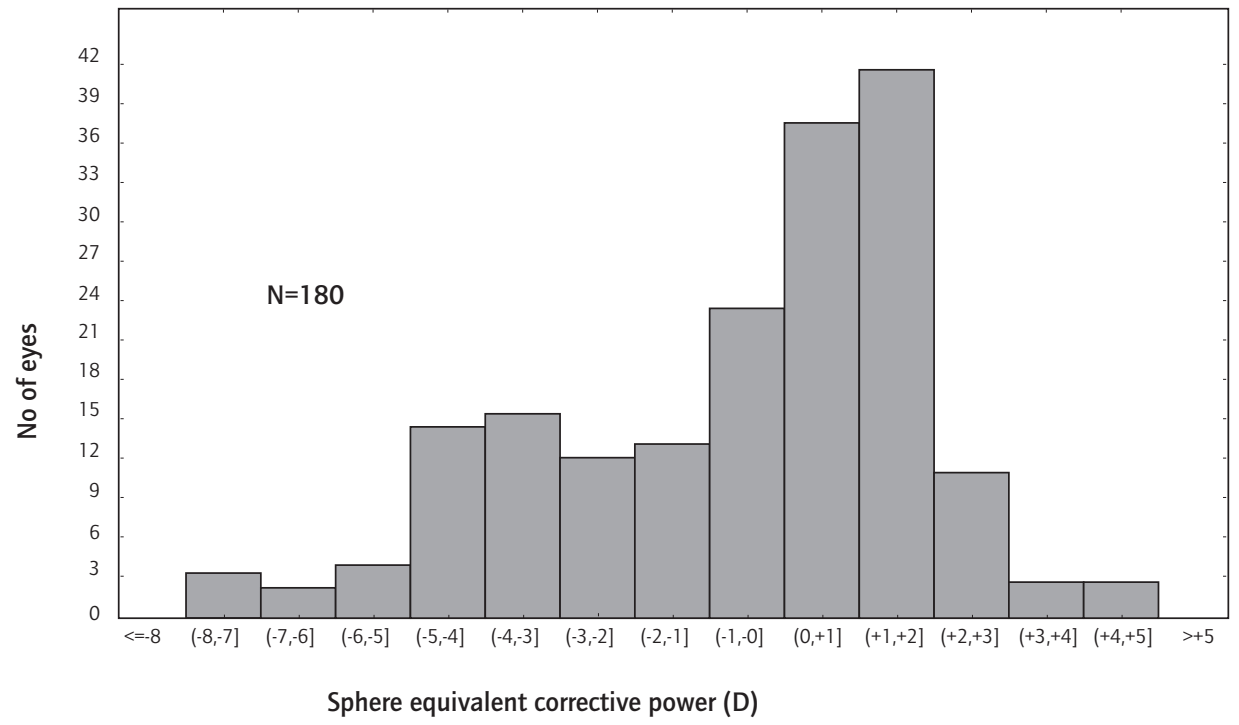

Figure 3. Distribution of ametropia (sphere equivalent corrective power at vertex) of 90 subjects (180 eyes).

from centre to periphery. By varying this profile we were able to create two groups of varifocal lens types, one with 3, the other with 4 different additions. Fig. 1 shows these profiles in relation to addition power and distance from the optical axis. The group of L1, L2 and L3 are designed with emphasis on large progressive zones, whereas the group S1 to S4 aims 


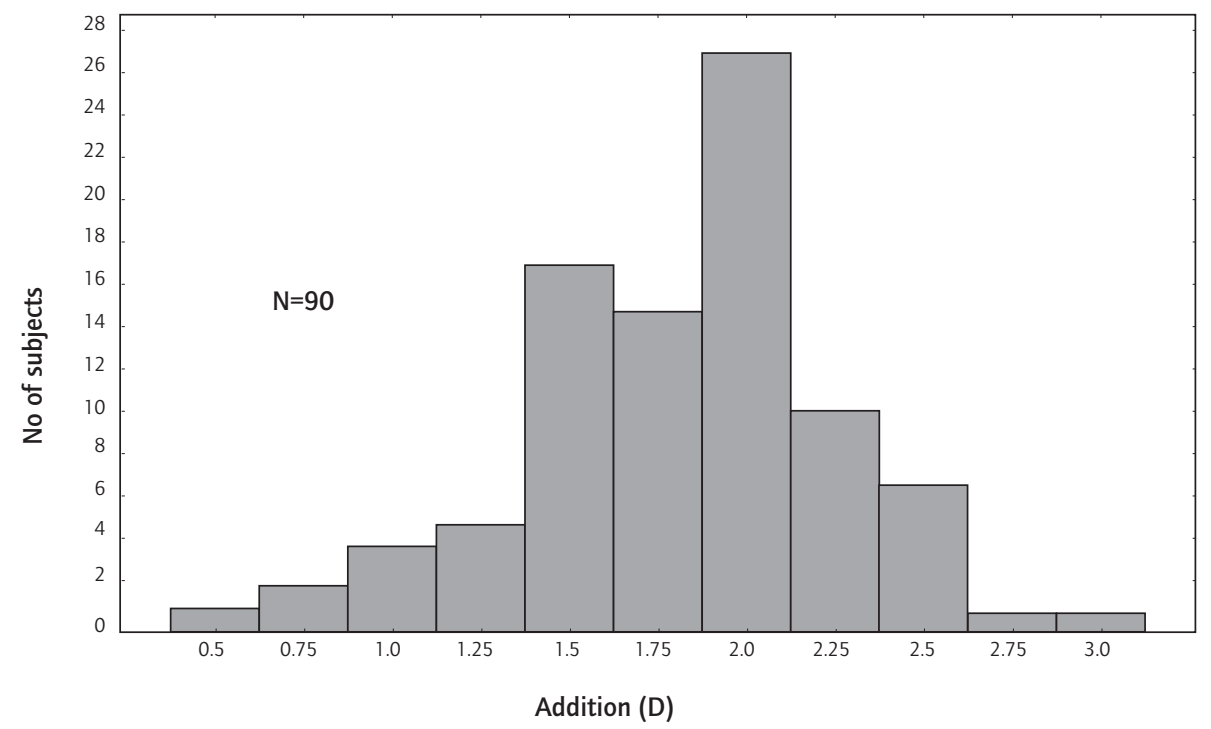

Figure 4. Distribution of needed addition (subjective refraction at $25 \mathrm{~cm}$ ) of 90 subjects that completed the study.

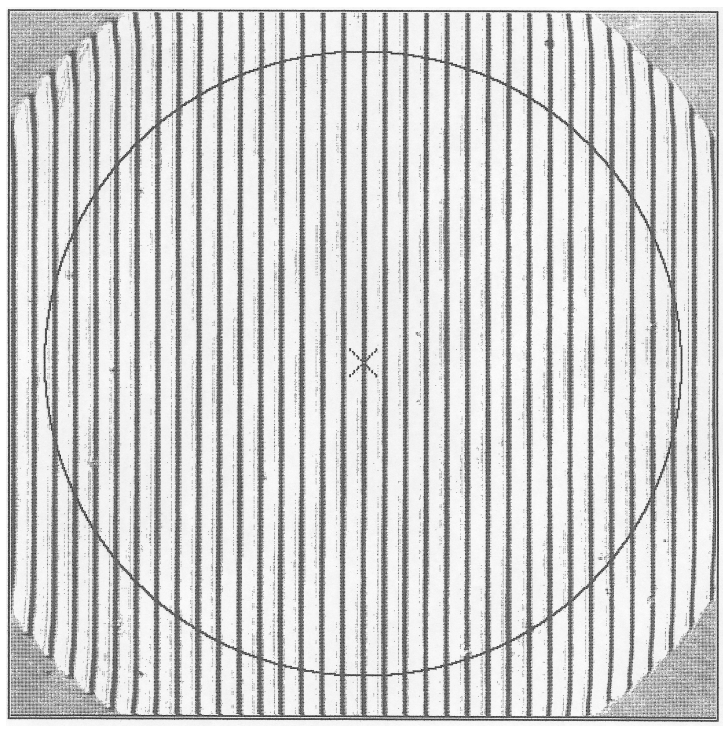

Figure 5. Interferometric fringe pattern of a plano reference lens 
to progressively reach near power in the centre of the lens. Preliminary testing showed that profiles L2 and L3 resulted in very low distance vision. We therefore decided to exclude these profiles from further experiments.

After production of each lens the power mapping was controlled by multiple wave interferometry, using a Fizeau-Tolansky interferometer. ${ }^{36}$ Figs. 5-7 show examples of the interferometric fringe patterns and the computer printout of the power distribution within the central $6 \mathrm{~mm}$ part of the lenses. Fig. 5 represents the fringe pattern of a plano lens as reference. Fig. 6 shows the analysis of a multifocal lens type $\mathrm{L} 1$ with a distance power of $-1.00 \mathrm{D}$. The addition starts at a distance $2 \mathrm{~mm}$ from the centre of the lens. In Fig. 7 an example of lens type $S$ is shown. Here the distance power is $-1.25 \mathrm{D}$ and the addition starts at $1.75 \mathrm{~mm}$ from the centre. Using this highly accurate inspection technique lenses that deviated more than $0.12 \mathrm{D}$ from the desired profile or with a RMS error (deviation from axi-symmetry) of more than $\lambda / 10$ were rejected.

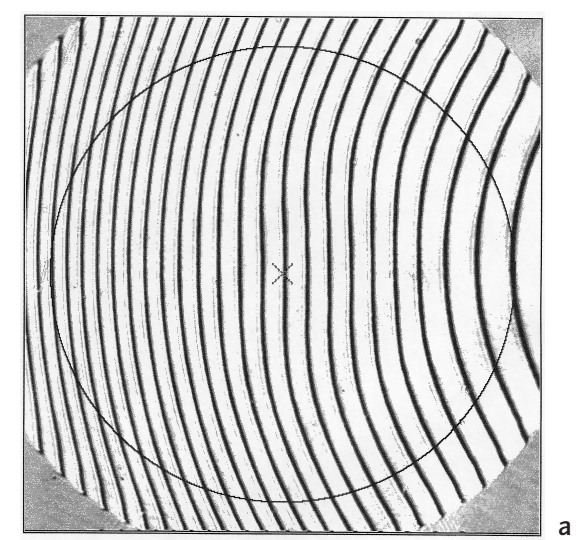

L1

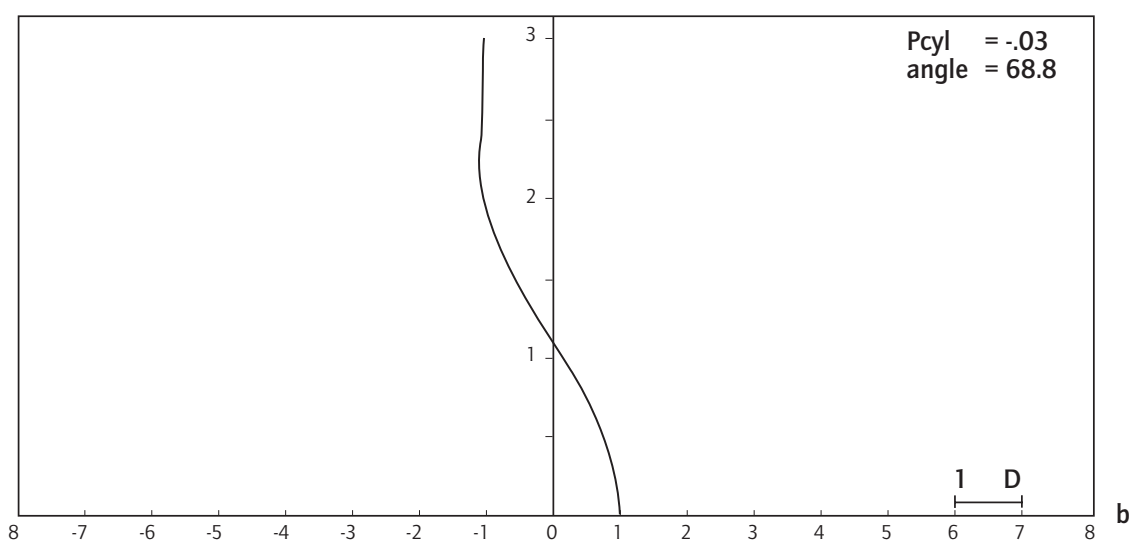

Figure 6. Interferometric fringe pattern (a) and power profile print-out (b) of a lens with distance power of - 1.00 $\mathrm{D}$ and an intermediate design principle (L1) 


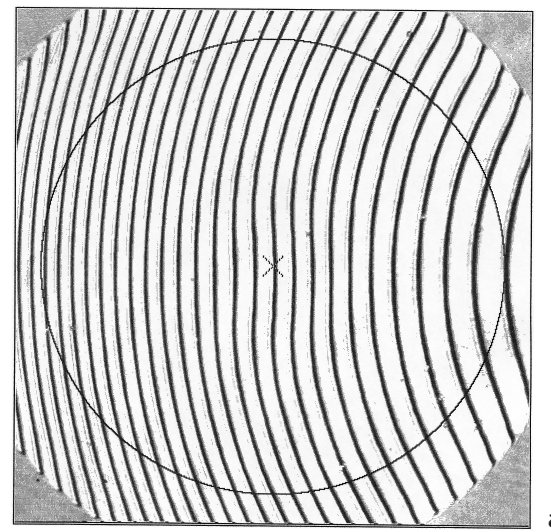

S3

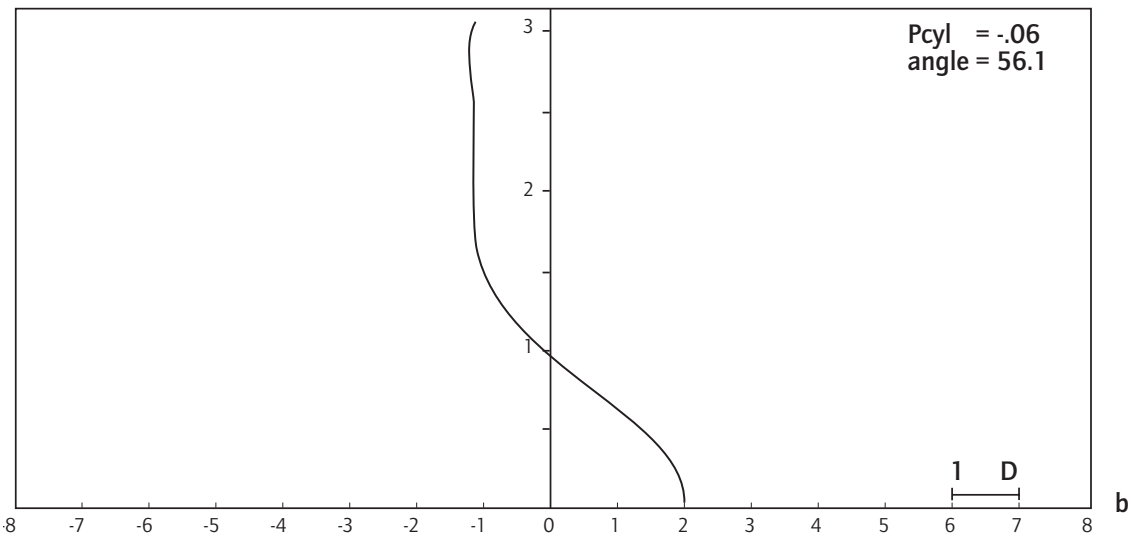

Figure 7. Interferometric fringe pattern (a) and power profile print out (b) of a lens with a distance power of - 1.25 $\mathrm{D}$ and a maximum power centre design principle (S3)

\section{Analysis}

The effects of lens design, addition, pupil size, lens centration relative to the pupil and lens design on visual acuity were studied. The influences of the lens design and of the measured independent factors on visual acuity were tested through multiway analysis of covariance. At the end of the experiments, each subject was allowed to use the lenses that provided the best subjective vision for three months. At the end of this period the patients were asked about their wearing time with the lenses, to subjectively rate the items as listed in Table 3 and whether they would continue wearing the lenses. 


\subsubsection{Results}

The overall acceptance rate at 3 months follow up, using the criterion of possible lens wear 8 hours/day, 6 days/week was $67.8 \%$. Distance vision acceptance rate was very high, $97.7 \%$. Acceptance rate for near vision was $74.4 \%$. Details are given in Table 3. It can be seen from this table that visual problems at near are the most common cause of failure with the prototyped multifocal contact lenses used in this study.

In distance vision we found no statistical significant effects ( $p>0.01)$ of pupil size, addition, and lens centration on visual acuity (Fig. 8). The only significant effect on distance vision was (as explained in material and methods section) lens optical profile.

The statistical analysis of the results in near visual acuity exhibits significant effects of lens design, pupil size, addition and lens centration. To be able to graphically show these findings we have divided the subjects in two groups of equal numbers (Fig. 9). The hypothesis for this analysis was that a large pupil or the need for a high addition might cause problems in near vision. The group "SMALL" has a small pupil combined with a low addition. The group "LARGE" has a relatively large pupil combined with the need for a higher addition. Fig. 10 shows the result of the analysis for near vision. It can be seen that a large decentration of the lens relative to the pupil or a large pupil combined with a high addition, seems to be related to lower performance in near vision. However, this relation is not sufficiently strong to allow a reliable prediction of individual patients acceptance from the initial measurements.

Table 3. Overall success-rate and reasons for failure after 3 months of wear. Criterion is wearing the lenses 8 hours/day, 6day/week without signs or symptoms listed.

\begin{tabular}{lcc}
\hline Signs and symptoms & Absolute N & Percentages \\
\hline & 2 & 2.2 \\
Dry eye feeling & 1 & 1.1 \\
Discomfort & 2 & 2.2 \\
Lack of Motivation & 1 & 1.1 \\
Unaccepted VA at distance & 22 & 24.4 \\
Unaccepted VA at near & 1 & 1.1 \\
Unaccepted VA distance and near & 29 & 32.2 \\
Failures & 61 & 67.8 \\
Successful & 90 & 100.0 \\
Total patients & &
\end{tabular}

\subsubsection{Discussion}

We have found that centre near designs of multi-focal contact lenses were also very successful in distance vision. However, this was only if the addition is reached progressively. Two prototyped lenses with a centre near design that have a gradual change in power over the entire central area reached very low visual results for distance vision (lenses L2 and L3, Fig. 1). 


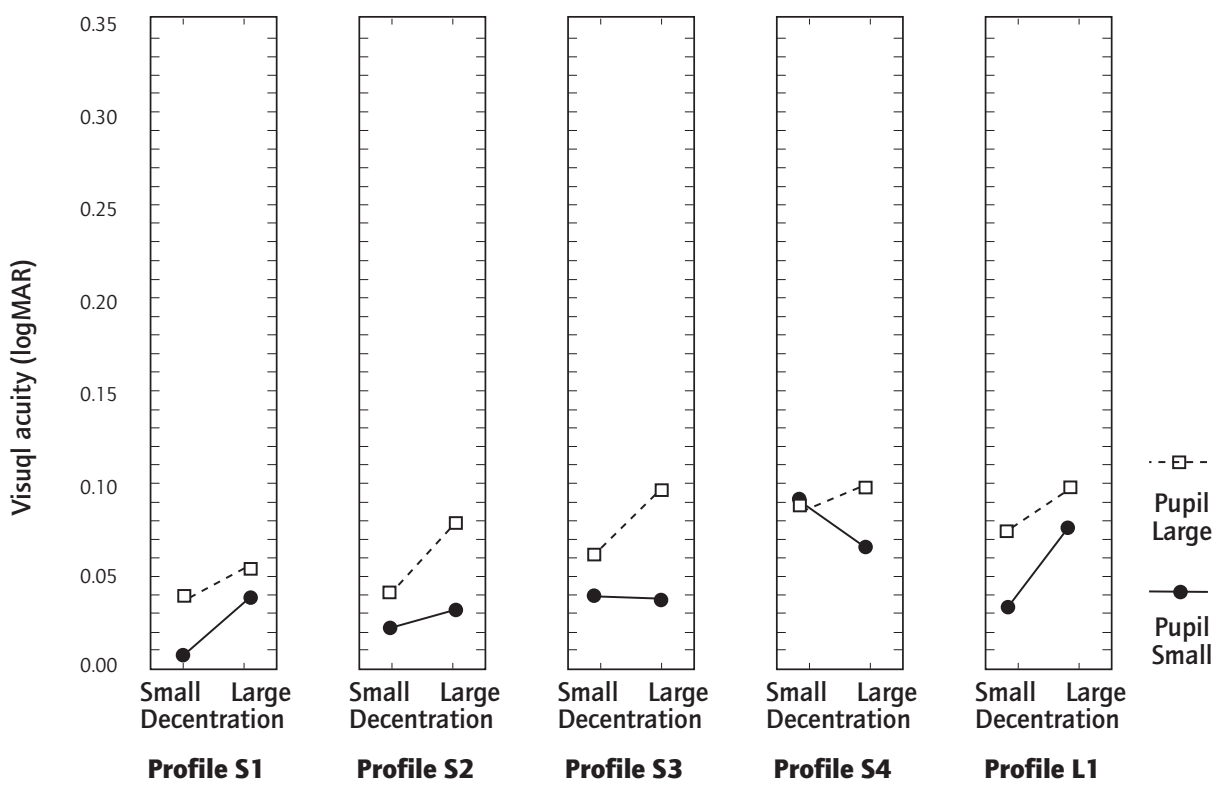

Figure 8. Results of visual acuity in distance vision related to the used lens profiles (5 columns) for small $(\bullet)$ and large () pupils in relation to small (left in each column) and large (right in each column) decentration. Effects on visual acuity (left scale) were not statistically different.

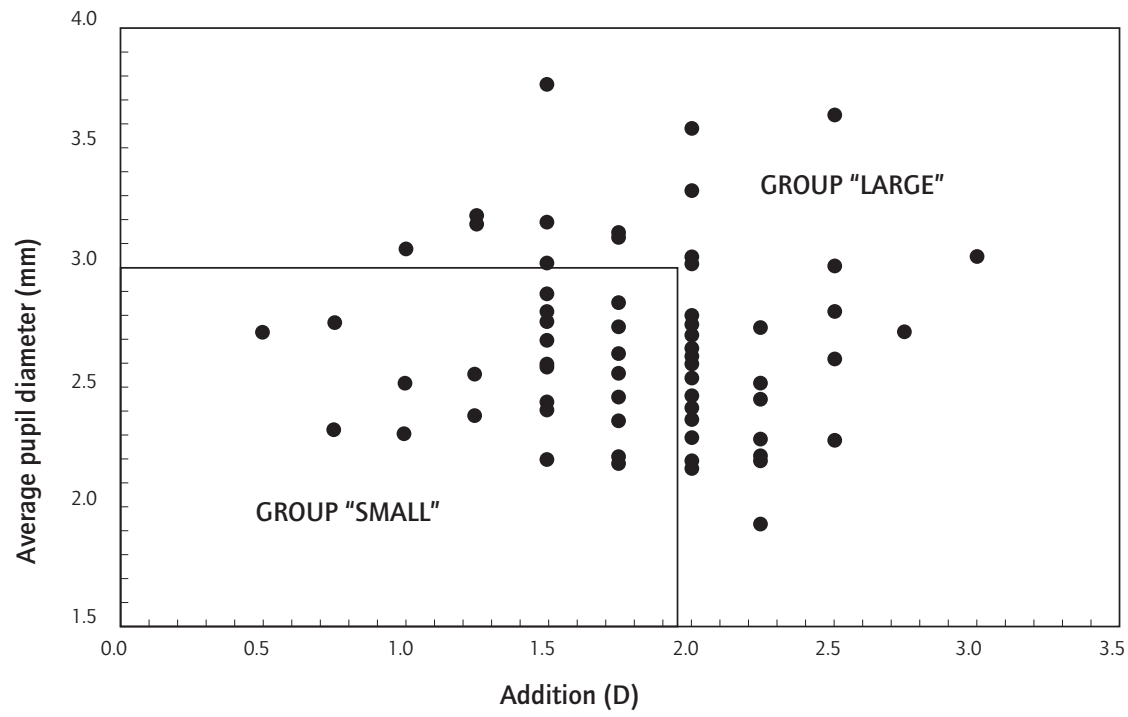

Figure 9. Grouping of the subjects under study for analysis in near vision. Group "SMALL" has a small pupil and low addition, whereas group "LARGE" has a large pupil with high addition. 


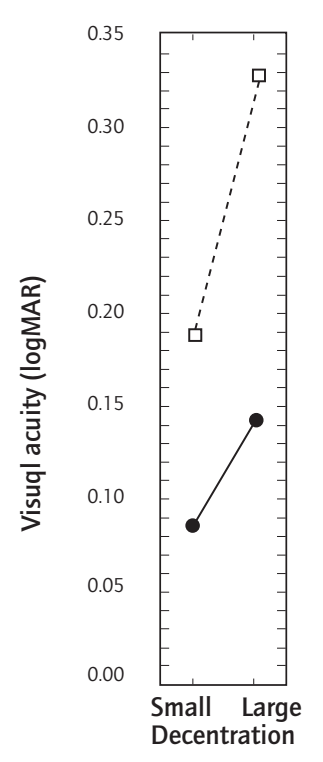

Profile S1

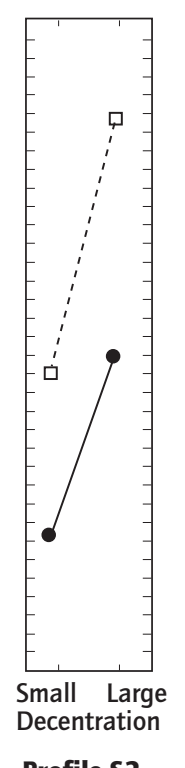

Profile S2

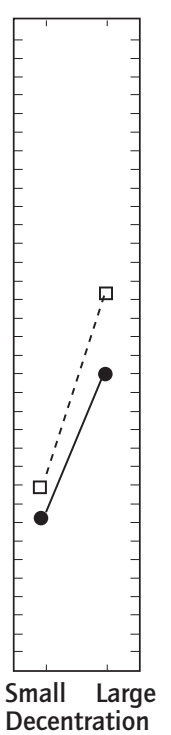

Profile S3

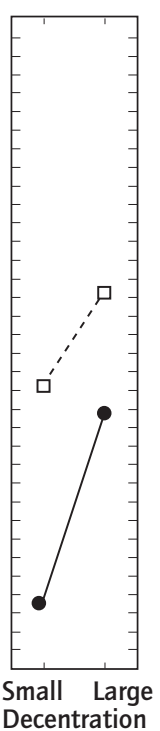

Profile S4

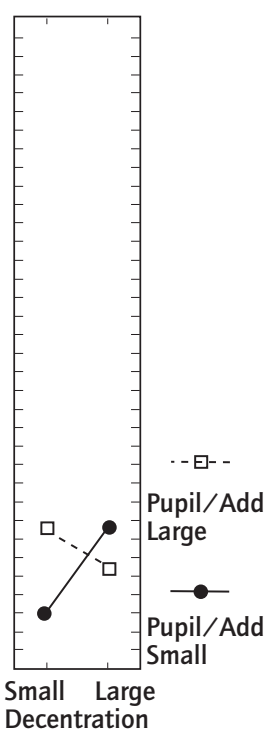

Profile L1

Figure 10. Results of visual acuity in near vision related to the used lens profiles (5 columns) for group "SMALL" having a small pupil and low addition $(\bullet)$ and group "LARGE" having a large pupil and high addition ( $(\square)$ in relation to small (left in each column) and large (right in each column) decentration. Effects on visual acuity (left scale) are statistically significant.

A possible explanation could be the fact that the retina needs a minimal level of difference in illuminance (intensity variation) within an image to be able to discriminate the desired image for further processing. Although in the varifocal lens all powers are available to the eye receptors, thus sharp images can be formed (are available) for all distances, the luminance flux of the images can vary dramatically. In this way a situation can exist in which the patients tries to concentrate on the available distance image but the (although blurred) image via the near power part of the lens contains enough intensity to prevent the retina (brain) discriminating the image for distance from the total images. The result being that everything is blurred for distance vision while near vision is perfect. Given that the optical quality of the lens is equal for all available powers the problem is (as known in photography) entirely due to aperture size. In case of a varifocal lens this means that the distribution of power areas available within the continuum of the desired focal distances is a critical factor. To make this clear let us compare lens designs S2 and $\mathrm{L} 2$ in Fig. 1. Both reach an addition power of $2.5 \mathrm{D}$ in the centre and 0 addition power at 2.0 $\mathrm{mm}$ from the centre. If we consider an average pupil diameter of $3.7 \mathrm{~mm}$ for distance vision under photopic luminance ${ }^{35}$ all powers are available to form images from distance to near. For near, addition powers from 2.0 to $2.5 \mathrm{D}$ are available up to $0.5 \mathrm{~mm}$ from the centre. The power area for near is about the same for both lenses and covers an area of $0.5^{2} \pi=0.79 \mathrm{~mm}^{2}$. For distance power the situation is entirely different. If we accept that a blur of $0.25 \mathrm{D}$ still contributes 
to the image for distance, lens $\mathrm{S} 2$ reaches that point at $1.12 \mathrm{~mm}$ from the centre meaning there is a ring shaped power area available of $\left(1.85^{2} \pi\right)-\left(1.12^{2} \pi\right)=6.81 \mathrm{~mm}^{2}$. This is a factor 8.6 more than in the near part. In lens $\mathrm{L} 2$ the $0.25 \mathrm{D}$ point is reached at $1.6 \mathrm{~mm}$ from the centre and consequently the available power area for distance in this area is only $\left(1.85^{2} \pi\right)-\left(1.6^{2} \pi\right)$ $=2.71 \mathrm{~mm}^{2}$ (factor 3.4 compared to near area). Lens S2 was not successful in distance vision. It seems that a factor 3.4 of intensity difference is not enough to discriminate between images for far and near. Since lens L1 was successful for both distance and near vision we might get close to finding the minimal needed factor to discriminate the images by computing the power area for that lens. The $0.25 \mathrm{D}$ point is reached at $1.38 \mathrm{~mm}$ giving a power area of $\left(1.85^{2} \pi\right)-$ $\left(1.38^{2} \pi\right)=4.77 \mathrm{~mm}^{2}$. The critical difference in image area necessary for image discrimination in a distance vision situation seems to lie around a factor 6 .

If we now apply the above hypothesis to the situation of near vision it appears at a first glance that the above developed hypothesis does not fit the case. Consider the situation of lens S2 but now in the situation the patient concentrates on near vision. The near part would have an area much smaller (factor 8.6) than available for distance vision. But the lens works on average well for near. Why? A part of the explanation is pupil size. With larger pupil sizes we found significantly lower performance at near. This is in accordance with the above theory. However, the average pupil size found for near vision under photopic luminance conditions is $2.7 \mathrm{~mm}^{35}$, much smaller than for distance vision. If we compute the areas available again using this data it shows that the near area remains $0.79 \mathrm{~mm}^{2}$ but for distance it is under reading conditions $\left(1.35^{2} \pi\right)-\left(1.12^{2} \pi\right)=1.79 \mathrm{~mm}^{2}$, a small factor of only 2.27 , but in the near vision condition in favour of the distance part. Now, why is a factor in distance as much as 6 needed to discriminate the image in distance vision and a factor of 2.27 in favour of the distance power not conflicting in near vision? The conclusion must be that the situation for near vision is entirely different than that of distance vision. A hypothesis could be that there is something like a "central optics first selection" in retinal processing if the difference in intensity between the images is small. There could be a relation with the efficiency of photonic energy transportation dependent on the angle of incidence on the receptors. An other explanation could be that spherical aberration of the eye-lens system plays a role in the sense that our allowance for $0.25 \mathrm{D}$ blur for the peripheral part of the lens means that the area for distance vision is further reduced and maybe under threshold. Prescribing contact lenses for presbyopic patients remains a difficult topic. The main concern is that it is very difficult to predict successful lens designs from biometrical measurements. This seems inherently connected with the "complex of presbyopic correction", in which optical principals, dynamic physiological optics, neuro-retinal processes and psychological factors of vision interfere. However, with the precisely manufactured and controlled optics of the varifocal lenses as used in this study the role of dynamic physiological optics of the lens-eye system and with that lens design can be (re)considered as of basic importance.

\subsubsection{Conclusions}

Many modes to correct presbyopia by contact lenses are today available. Although ingenious optically most remain unpredictable physiologically. The correction of presbyopia by con- 
tact lenses is very complex. The use of the seven areas in the presbyopia contact lens complex as described in this study could be of help in guiding further research.

The centre near varifocal lens clinical study did not show significant effects of pupil size, lens centration or addition on distance vision. The only significant factor was lens design. Overall acceptance was $67.8 \%$. For near vision we have found large decentration relative to the pupil and large pupils with a need for high addition to be significant negative factors. The design approach of combining numerical simulations with measure of clinical performance, as used with the prototyped lenses in this study, has shown to be a promising concept in designing varifocal soft contact lenses.

\section{References}

1. Feinbloom W. Segmented optics in scleral lenses to correct presbyopia. United States Patent. US, 1938.

2. Williamson-Noble F. Contact Lenses - what of the future? Br. J. Physiol. Optics 1951;8:244-6.

3. De Carle JT. Bifocal and multifocal contact lenses. Contact lenses: a textbook for practitioner and student, 3 ed. Edited by Stone J. London, Butterworths, 1989.

4. Josephson JE, Caffery B. Bifocal hydrogel lenses: an overview. J Am Optom Assoc 1986;57:190-5.

5. Lindridge C. Far and Near-bifocal and multifocal contact lenses. Contact Lens Journal 1987;16:250-3.

6. Stein HA. The management of presbyopia with contact lenses: a review. CLAO J 1990;16:33-8.

7. Davies I, Papas E. The Correction of Presbyopia with Soft Contact Lenses. Optician 1990:14-22.

8. Back A. Presbyopes and contact lenses is there hope? Optician 1991;july 5:16-9.

9. Farkas P. Breaking the Contact Lens Barrier. Optom Economics 1990:28-31.

10. Krajewski R. Presbyopic Lenses: An Update. Contact Lens Forum 1989:40-1.

11. Evans J, Thompson D. An Overview of Bifocal Contact Lenses. J BCLA 1991;14:71-4.

12. Lutz P, Parendeau P. Multifokale Kontaktlinsen: Markt- und Verhaltensanalyse, AnpassungsRaetschlage. Kontaktlinse 1996;30;7/8:13-7.

13. Bridgewater BA. Harte gaspermeable Multifokallinsendesigns. Kontaktlinse 1993:5-9.

14. Josephson JE, Erickson P, Back A, Holden BA, Harris M, Tomlinson A, Caffrey BE, Finnemore V, Silbert J. Monovision, A proposed position paper. J Am Optom Assoc 1990;61:820-6.

15. Loshin D, Loshin MGC. Binocular summation with monovision contact lens correction for presbyopia. Int. Contact Lens Clin 1982;9:161-65.

16. McGill E, Erickson P. Monovision adaptation and fixation disparity. Contact lens J 1989;17;6:182-5.

17. Plainis S, Charman WN. On-eye power characteristics of soft contact lenses. Optom Vis Sci 1998;75:44-54.

18. Bradley A. Foreword: Simultaneous Bifocal and Multifocal Vision: From Theory to Practice. Optom Vis Sci 1993;70:437-8.

19. Morrison R. Simultaneous imagery soft bifocal contact lenses. BCLA congress proceedings 1989.

20. Cohen AL. Diffractive bifocal lens designs. Optom Vis Sci 1993;70:461-8.

21. Klein SA. Understanding the diffractive bifocal contact lens. Optom Vis Sci 1993;70:439-60.

22. Jones $B$, Lowther $G$. The effect of near zone size of a center-near zone hydrogel contact lens bifocal on visual acuity. ICLC 1989;16:87-93. 
23. Eggink F, Pinkers A, De Graaf R. Visual acuity and contrast sensitivity with Diffrax Contact Lenses. Contact Lens Journal 1990;18:37-9.

24. Loshin D. The holographic/diffractive bifocal contact lens. ICLC 1989;16:77-86.

25. Sheedy JE, Harris MG, Gan CM. Does the presbyopic visual system adapt to contact lenses? Optom Vis Sci 1993;70:482-6.

26. Parendeau P, Kress J, Lutz P. Die Bedeutung der Hirnselektivitaet bei Multifokallinsen. Kontaktlinse 1993:5-10.

27. Back A, Hoods R, Holden B. The comparative visual performance of monovision and various concentric bifocals. BCLA Meeting, 1987.

28. Blanks G. Literature Review of Hydrogel Presbyopic Correction. ICLC 1991;18:142-7.

29. Back A, Grant T, Hine N. Comparative visual performance of three presbyopic contact lens corrections. Optom Vis Sci 1992;69:474-80.

30. McGill E, Erickson P. Stereopsis in presbyopes wearing monovision and simultaneous vision bifocal contact lenses. Am J Optom Physiol Opt 1988;65:619-26.

31. Gagnolati W. Acceptance of different multifocal contact lenses depending on the binocular findings. Optom Vis Sci 1993;70:315-22.

32. Baude D, Miege C. Presbyopia compensation with contact lenses - a new aspheric progressive lens. J BCLA 1992:7-15.

33. Zandvoort SW, Kok JH, Molenaar H. Good subjective presbyopic correction with newly designed aspheric multifocal contact lens. Int Ophthalmol 1993;17:305-11.

34. Terry RL, Schnider CM, Holden BA, Cornish R, Grant T, Sweeney D, La Hood D, Back A. CCLRU standards for success of daily and extended wear contact lenses. Optom Vis Sci 1993;70:234-43.

35. Chateau N, de Brabander J, Bouchard F, Molenaar H. Infrared pupillometry in presbyopes fitted with soft contact lenses. Optom Vis Sci 1996;73:733-41.

36. Baude D. Caracterisation, optimation et realisation de composants optique multifocaux diffractifs et refractifs. Thesis, Université de Paris-Sud, Paris, 1990. 


\subsection{Aspheric Contact Lenses in High Ametropia}

Contrast Sensitivity with Soft Contact Lenses Compensated for Spherical Aberration in High Ametropia

John de Brabander ${ }^{1}$, Nicolas Chateau ${ }^{2}$, Florence Bouchard ${ }^{2}$ and Sandrine Guidollet ${ }^{3}$.

From:

1 Eye Research Institute Maastricht, Department of Ophthalmology Academic Hospital, University Maastricht, the Netherlands.

2 Ocular Sciences (formerly Essilor CL division), R\&D International, Créteil, France.

3 Université de Paris-Sud, Orsay Cedex, France.

Optom Vis Sci 1998;75;1:37-43. 
Abstract

Background. In optical systems, it is usual to compensate for longitudinal spherical aberration. In order to increase image quality, lens surfaces can be made aspheric to bring all object light rays into focus at the image plane. Theoretically, soft contact lenses with high power and spherical surfaces show significant amounts of spherical aberration. The use of spherical aberration-free soft contact lenses could therefore improve retinal image quality in the case of high ametropia. However, because of ocular aberration, accommodation effects, pupil dynamics, contact lens flexure and positioning, the computation of the spherical aberration induced when a contact lens is placed on the eye is complicated.

Methods. In this study, the spatial contrast sensitivity (CS) of 61 high ametropes wearing soft contact lenses with, and without, in-air spherical aberration compensation is measured.

Results. A slightly better overall performance was found with the standard lenses. There was no significant influence by the type of ametropia, age, and gender. If individual results are considered, clinically significant differences between the two lens groups are observed in approximately $30 \%$ of the cases.

Conclusion. It seems that correcting in-air soft contact lens spherical aberration systematically is of no clinical interest. However, selective manipulation of spherical aberration could, in high power soft contact lenses, significantly improve CS in individuals.

Key words:

Contrast sensitivity, spherical aberration, soft contact lenses, high ametropia. 


\subsubsection{Introduction}

In conventional contact lenses, the spherical shape of both anterior and posterior optical surfaces induces longitudinal spherical aberration. With lenses of higher back vertex power (BVP), previous calculations ${ }^{1-5}$ and in vitro measurements ${ }^{6}$ suggested that the level of spherical aberration could significantly degrade the retinal image.

A literature review reveals disagreements between attempts ${ }^{1-4}$ to predict the in vivo contact lens spherical aberration by computer simulations. The proposed theoretical approaches differ in the representation of the corneal surface, and in the treatment of the flexure effects in soft contact lenses. To our knowledge, these predictive models have not been checked against in vivo measurements of soft contact lens induced spherical aberration, although a method for making such experiments was demonstrated with varifocal contact lenses. ${ }^{7}$

More conventional visual testing techniques were used in studies $3,8,9$ which addressed the effects of corrective spectacle lens and soft contact lens spherical aberration on visual performance. These studies show disagreement on the effects of contact lens spherical aberration on high contrast visual acuity (VA). However, it has been found that evaluation of optical and neural contributions to visual performance in aging of the eye ${ }^{10}$, binocular vision ${ }^{11}$, and monovision ${ }^{12}$ can be improved by the assessment of contrast sensitivity (CS) to a range of spatial frequencies. In these studies, differences in CS were found that could explain typical problems with image quality or visual comfort reported by patients with a normal high contrast VA. Similar conclusions were reached in studies evaluating or comparing the visual performance of patients wearing different types of contact lenses or in evaluating formation of soft lens deposits. ${ }^{13}$

In a review of CS assessment and applications, Morrison ${ }^{14}$ points out that special attention needs to be given to the methodology, instrumentation, and test conditions. Referring to the basic work of Campbell and Green ${ }^{15}$, Eldred and Jose ${ }^{16}$ state that binocular summation seems to be responsible for a $42 \%$ higher CS, across all spatial frequencies in normal observers. In conditions like monovision, unequal monocular luminance, stereo blindness, weak accommodative vergence cues, low vision, and amblyopia, retinal rivalry inhibition processes can have influences on different parts of the CS curve function. As described above, there are many variables that influence CS; this means that, especially in studying CS in a group of high ametropes, care should be taken in methodology, use of quality-controlled new lenses, patient selection, and/or grouping of patients for statistical analysis.

Cox and Holden ${ }^{17}$ evaluated the CS of subjects wearing soft contact lenses, which were aspherized in order to induce various levels of negative and positive aberrations, including a nonaberrated optical profile. The best average performance was obtained with the nonaberrated lens; typically high contrast VA was not affected. In this study, artificial pupils were introduced by means of diaphragms in a trial frame. No significant changes in CS were found with 3-mm artificial pupils. With 6-mm artificial pupils, significant reductions in CS were found for aberrated lenses; positive aberration induced a significantly greater loss of CS than equivalent negative aberration. All the lenses in this study were designed with plano paraxial power, and were thus suited for experimenting with emmetropic or low ametropic 
subjects. Using a similar protocol, Collins et al. ${ }^{18}$ reached approximately the same conclusions in a study with rigid contact lenses.

Including reference to their theoretical calculations and discussion, a rough extrapolation of these former results would suggest that the compensation of spherical aberration in higher power lenses could improve the visual performance of high ametropes. However, this assumption was not verified experimentally with highly ametropic contact lens wearers. The interest in considering such a hypothesis has been renewed by recent improvements in lathe machining technologies, which greatly simplified the fabrication of arbitrary aspheric lens surfaces. The investigation described in the current paper was aimed to answer the following question: Does the systematic correction of soft contact lens in-air longitudinal spherical aberration significantly improve monocular and/or binocular spatial CS in high ametropes?

\subsubsection{Methods}

Our study followed a two-center randomized cross-over double-masked protocol. A minimum of 25 myopes and 25 hyperopes was to be recruited according to the following criteria: age between 15 and 50 years, refractive cylinder $<0.75 \mathrm{D}$, monocular high contrast VA $>0.5(20 / 40)$ decimal notation, no active eye disease or visual system-related systemic disease, no degenerative changes, and no contraindication for soft contact lens wear. Subjects were informed about the study by written information and entered the study after oral consent.

All subjects underwent an initial optometric examination followed by a contact lens fitting procedure, where base curve radii and contact lens BVP were determined. Trial lenses had a diameter of $14 \mathrm{~mm}$, with available base curves of 8.3 to $9.2 \mathrm{~mm}$ (0.3-mm steps), in $70 \%$ water content MMA-NVP material. Good fit criteria involved 0.5 to $1.0 \mathrm{~mm}$ movement on blinking, with centering such that the lens edge extended more than $0.5 \mathrm{~mm}$ from the visible iris at any angle. BVP was determined using the sphere equivalent highest plus/best high contrast VA method. The test eye for monocular experiments was also determined as the one offering best high contrast VA, or as the most ametropic if acuities were found to be similar in both eyes.

At the end of this first visit, the subjects were trained to the CS testing method by performing three measurements at 6 cpd under binocular conditions. The CS testing method consisted of a temporal, two-alternative forced choice, tracking procedure. ${ }^{19}$ The vertical sinusoidal grating stimuli were generated on a monitor by a Cambridge Research System VSG 2 card with specially developed software. Before testing, the stimuli generators were calibrated by creating look-up table files, using an optical luminance meter connected to the VSG 2 card. Screen luminance was $100 \mathrm{~cd} / \mathrm{m}^{2}$, room illuminance 10 to $20 \mathrm{~cd} / \mathrm{m}^{2}$. Further details of the test conditions are listed in Table 1.

For each subject, two pairs of prototypal soft contact lenses were cut in $70 \%$ water content MMA-NVP copolymer using a Euro Precision Technology (EPT, Almelo, the Netherlands) submicron lathe machine. This lathe is able to produce almost any aspheric surface with 
Table 1. Parameters and test conditions for VSG 2 CS measurements.

$\begin{array}{ll}\text { Distance, subject to screen } & 2 \mathrm{~m} \\ \text { Room illuminance } & 10-20 \mathrm{~cd} / \mathrm{m}^{2} \\ \text { Average luminance of screen } & 100 \mathrm{~cd} / \mathrm{m}^{2} \\ \text { Screen frequency } & 50 \mathrm{~Hz} \\ \text { Fixation target (central disk) } & \text { luminance } 5 \mathrm{~cd} / \mathrm{m}^{2} \text {, diameter } 0.075^{\circ} \\ \text { Object presentation time } & 0.5 \mathrm{sec} \\ \text { Interval between two presentations } & 0.75 \mathrm{sec} \\ \text { Allowed responds time } & 2 \mathrm{sec} \\ \text { Tested spatial frequencies } & 6 \mathrm{and} 15 \mathrm{cpd} \\ \text { Initial contrast } & 10 \% \\ \text { Change contrast after } & 3 \mathrm{correct} \text { answers or } 1 \text { wrong answer } \\ \text { Initial descending step } & 0.32 \mathrm{log} \text { units } \\ \text { Change in step value } & 50 \% \text { of former value } \\ \text { End of test at step value } & 0.04 \mathrm{log} \text { units } \\ \text { Max. number of presentations } & 59\end{array}$

high precision and a surface roughness of only 20 to $25 \mathrm{~nm}$. Consequently the lenses need no polishing, which would spoil the shape of the surface. In the first pair, a standard lens design with spherical back and front surfaces was used. In the second pair, the spherical back surfaces were made similar to those of the first pair, but with front surfaces aspherized in order to compensate for in-air spherical aberration. In the latter lenses, the shape of the aspheric surface is calculated with a standard ray tracing procedure. ${ }^{20}$

After hydration in saline solution, the longitudinal aberration of each lens was assessed in vitro, using a computerized wet-cell Fizeau-Tolansky interferometer. ${ }^{20}$ This technique offers sharply defined multiple-wave interferograms, which allow accurate automated fringe detection (see Figs. 1 and 2, top). A phase retrieval algorithm is used to obtain the lens wavefront aberration. The longitudinal spherical aberration is then calculated in the form of a high order polynomial (Figs. 1 and 2, bottom). The measured power distributions were compared to the theoretical longitudinal spherical aberration; any local difference larger than $0.12 \mathrm{D}$ or wavefront standard deviation larger than $\lambda / 10$ led to lens rejection.

At a second visit, the binocular and monocular CS was measured at a viewing distance of 2 $\mathrm{m}$, with both types of lenses in a randomized order, allowing each lens $10 \mathrm{~min}$ to stabilize. To avoid fatigue, CS was measured at spatial frequencies of 6 and $15 \mathrm{cpd}$. These values were chosen to make it possible to compare CS at medium (6) and high (15) spatial frequency. At first, CS was measured twice under binocular condition. If the difference between the two measurements revealed $>0.1 \mathrm{log}$ units, another measurement was taken, and only the two closest values were used. The same procedure was repeated under the monocular condition.

While answering the test, the subjects were wearing a trial-lens frame containing the spherical over-refraction suited to each pair of lenses, giving highest high contrast VA with highest plus power at $6 \mathrm{~m}$. This distance (larger than the test distance) was chosen to make sure the best focus would not be in front of the retina at the CS test distance. If over-refraction 

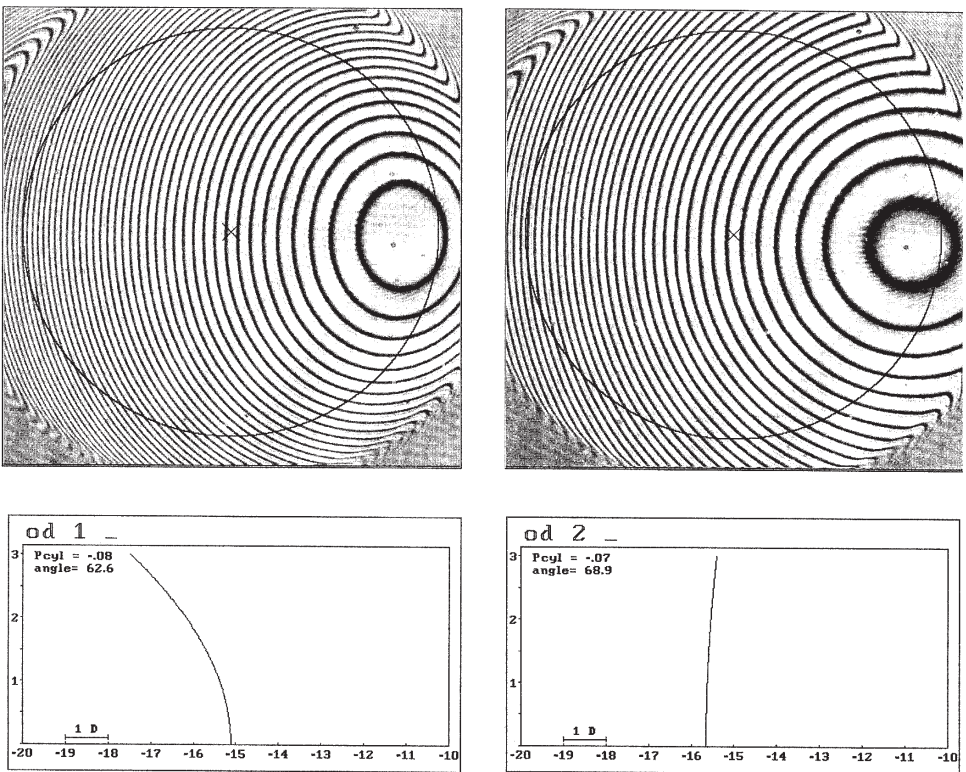

Figure 1. Fringe patterns (top) and power profiles up to $3 \mathrm{~mm}$ from center (bottom) of a standard aberrated (left) and an aspheric corrected (right) high minus soft contact lens, as measured with a computerized wet-cell Fizeau-Tolansky interferometer.
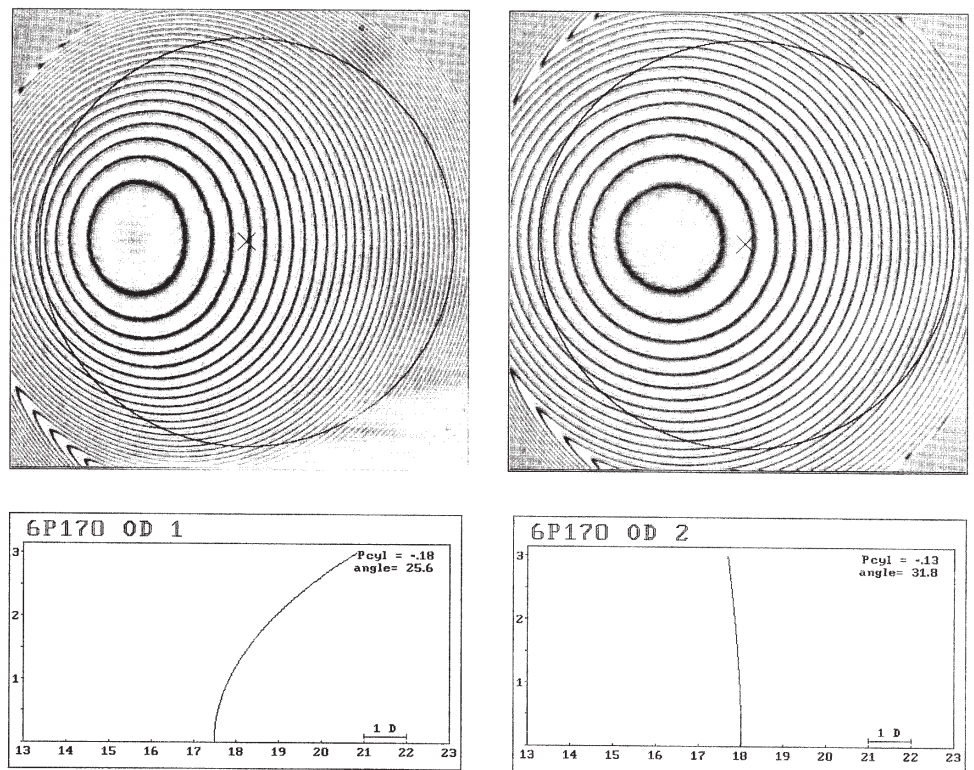

Figure 2. Fringe patterns (top) and power profiles up to $3 \mathrm{~mm}$ from center (bottom) of a standard aberrated (left) and an aspheric corrected (right) high plus soft contact lens, as measured with a computerized wet-cell Fizeau-Tolansky interferometer. 
exceeded plus or minus $1.00 \mathrm{D}$ a new lens, incorporating the power adjustment, was produced. If no additional power was needed, a plano trial frame lens was placed.

The results were unmasked after the end of the clinical experiments. Comparison of the data was done through an analysis of variance with two independent variables (type of ametropia and gender), one covariate (age), and 4 repeated factors (type of lens, spatial frequency, monocular/binocular vision, and measurement repetition).

\subsubsection{Results}

A total of 61 subjects ( 33 males, 28 females; 33 myopes, 28 hyperopes) with an average age of 30.5 years (range 15.1 to 50.0 years, SD 8.6) participated in the study. The histogram of Fig. 3 represents the age distribution. Ametropia ranged from -17.75 to $+20.25 \mathrm{D}$ with an average of - $0.79 \mathrm{D}$ (SD 9.66). The histogram of ametropia is represented in Fig. 4.

Comparison of reached high contrast Snellen VA of the individual eyes, while wearing standard (aberrated) or aspheric (corrected) soft contact lenses, revealed that differences typically remained within one line of acuity.

In Fig. 5, A to D, the results of the contrast sensitivity measurements are given. Pairing of this figure makes it possible to compare the results with corrected vs. aberrated lenses for myopia/hyperopia, monocular/binocular, and for frequencies of 6 and $15 \mathrm{cpd}$. The scaling ( $\log C S$ ) is fixed in relation to the tested frequency.

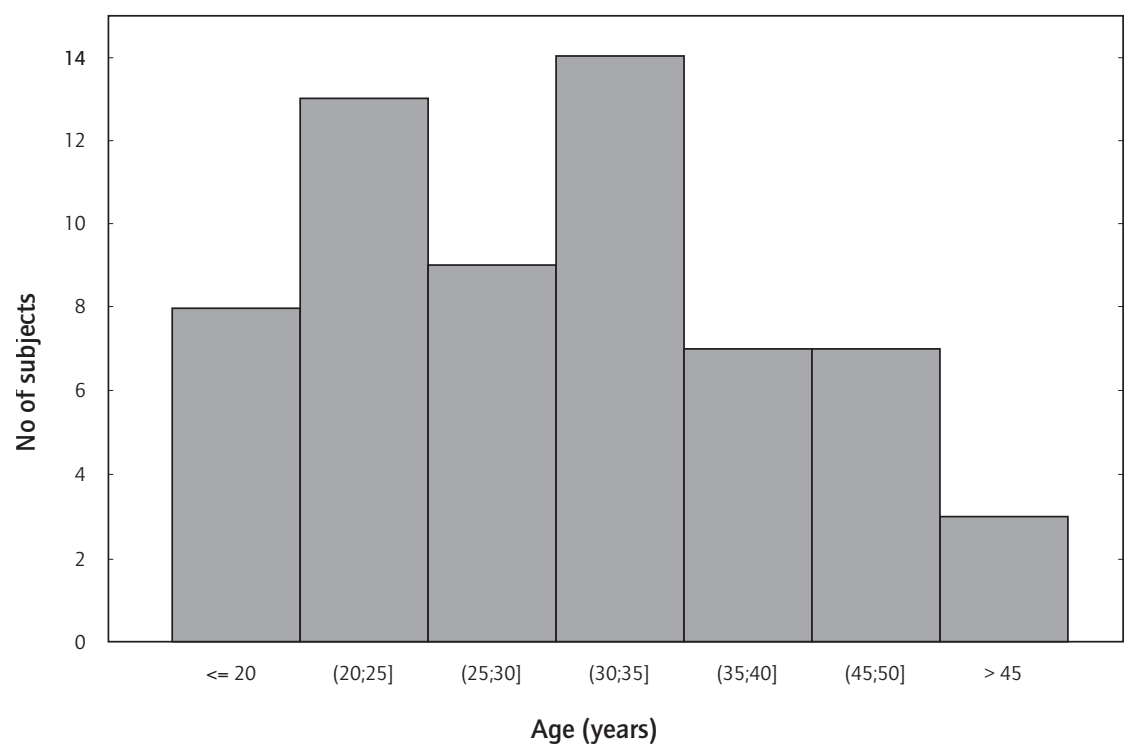

Figure 3. Distribution of age $(\mathrm{N}=61)$. 


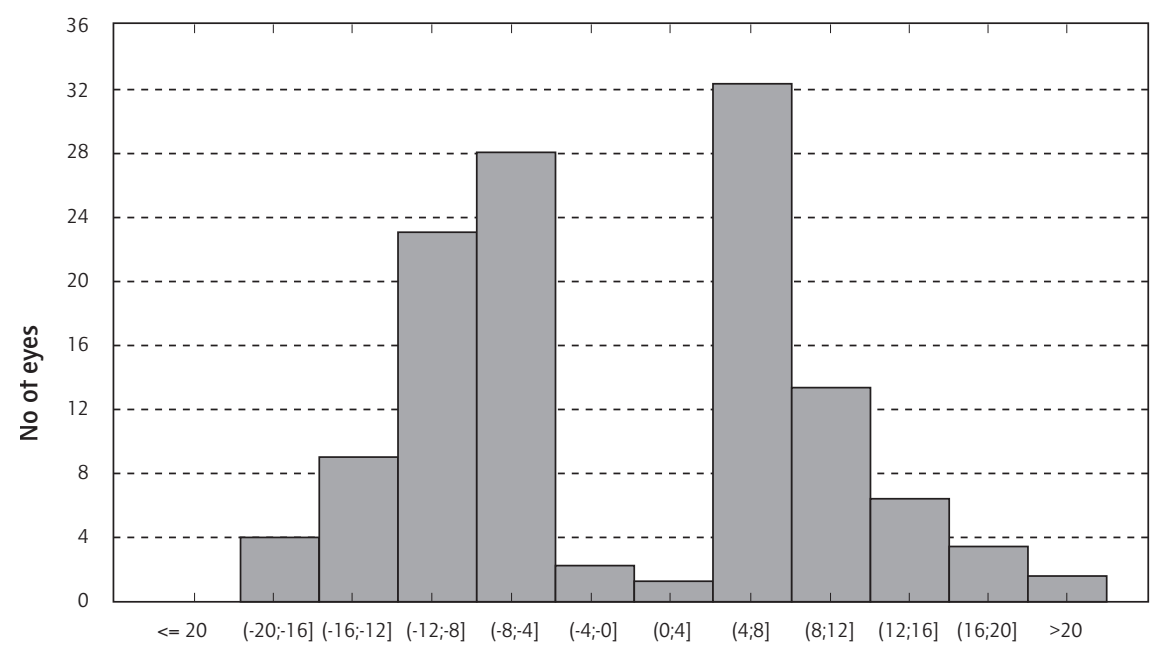

Sphere equivalent power (D)

Figure 4. Distribution of ametropia, vertex distance $0 \mathrm{~mm}(\mathrm{~N}=122)$.

The CS results ( Figs. $5 \mathrm{~A}$ to D) suggest a slightly better overall performance of the standard (aberrated) lenses. The average differences remain inferior to $0.1 \mathrm{LogCS}$. The analysis of variance confirmed that this tendency was statistically significant $(\mathrm{p}=0.015)$.

The analysis reveals no significant influence by the type of ametropia, age, or gender, and also no significant interaction between lens and ametropia effects.

Average binocular CS taken over all groups was $>0.15 \log$ units higher than average monocular CS (range of increase 0.17 to $0.31 \log$ units, $\mathrm{p}<0.001$ ). We also found a significantly higher increase in binocular compared to monocular $\mathrm{CS}$ with the lenses corrected for spherical aberration (lens/vision interaction $\mathrm{p}<0.02$ ). As can be seen from Fig. 6, this effect originates entirely from the results obtained at 6 cpd under binocular conditions.

Taken into account the bias by our selection of two closest measurements in some of the cases, the repeatability of CS measurement was generally acceptable (SD 0.06 LogCS in average). The between-subject variability was rather high (SD 0.25 LogCS typically).

\subsubsection{Discussion}

The between-subject variability as found in our study is higher if compared to variability in young subjects. ${ }^{10,14}$ However, if our age range is taken into consideration it reflects typical values also found by others. ${ }^{10,21}$ Fatigue and tear layer disturbances can influence intra-individual CS results. In our study, we instructed the subjects to blink normally during the testing; especially when "staring at the screen" was observed, we reminded the subject of this. Fatigue can never be completely avoided, but testing in a random order and some time of 

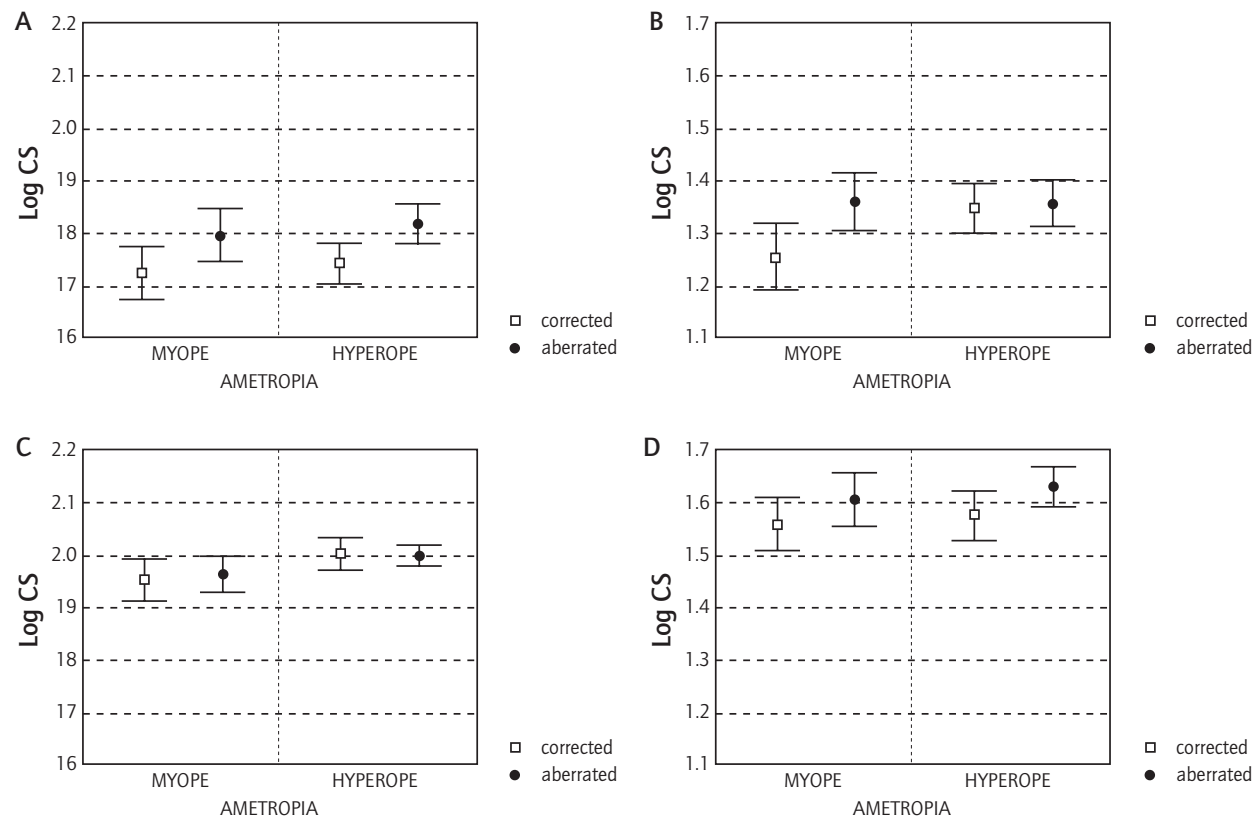

Figure 5. Average and SD of CS measurements on standard soft contact lenses with spherical aberration $(\bullet)$ and lenses of same parameters but with (front surface) corrected aberration (ם). Scaling is in accordance with tested spatial frequency. The results can be compared related to the tested spatial frequency at $6(A, C)$ and $15(B, D) c p d$ and/or under monocular ( $A, B)$ or binocular $(C, D)$ viewing conditions. Each section differentiates between myopia and hyperopia. A: Monocular CS measured at $6 \mathrm{cpd}$. B: Monocular CS measured at $15 \mathrm{cpd}$. C: Binocular CS measured at $6 \mathrm{cpd}$. D: Binocular CS measured at $15 \mathrm{cpd}$.

rest (15 min., for changing and settlement of the lenses) between tests seems to keep intraindividual variability at reasonable levels.

Not all subject showed a higher binocular CS than monocular. This can be explained from the fact that high ametropes are a "suspected" group in terms of development of the visual system, especially binocular vision (weak summation or inhibition). This could explain that our results show at average a lower CS increase percentage for binocular vision than normally found. ${ }^{11,12,15}$ An other possible explanation for the lower CS increase percentage for binocular vision could be a learning effect. Although we have allowed all subjects in the same manner to get used to the test, it could be that subjects (some more than others) could benefit from the fact that monocular testing always followed binocular testing. The next part of this discussion, and the fact that aberrated and corrected lenses were tested in a random order would, however, weaken the explanation that the relatively lower CS increase in binocular vision that we have found could be attributed to learning effects.

Furthermore, our results suggest that binocular enhancement is higher with soft contact lenses corrected for spherical aberration. However, as can be seen from the slopes of Fig. 6, this only occurred for the frequency $6 \mathrm{cpd}$, and this interaction originates entirely from the results obtained under the binocular condition. We should, therefore, be careful with this 


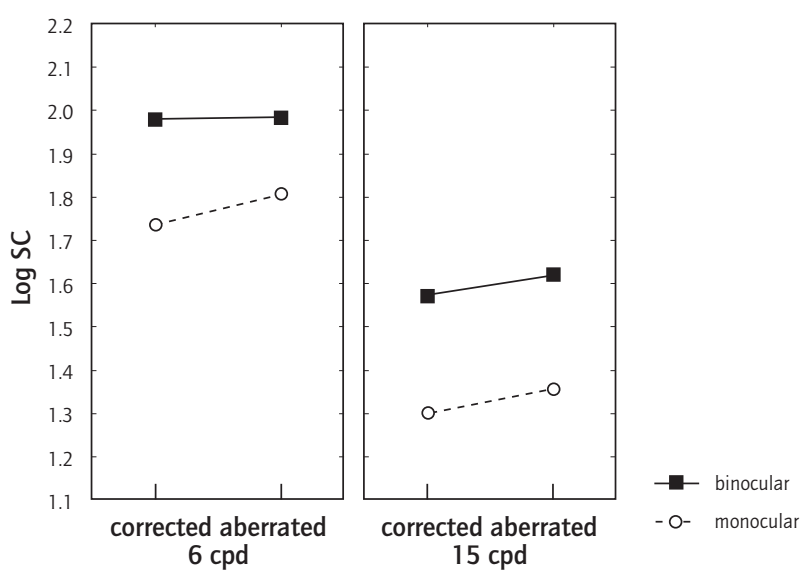

Figure 6. CS at 6 (left) and 15 (right) cpd under monocular (o) and binocular ( $\mathbf{- 1}$ ) test conditions for soft contact lenses corrected for in-air spherical aberration (corrected) and normal soft contact lenses with spherical aberration (aberrated). Note the different slope for binocular vision at $6 \mathrm{cpd}$.

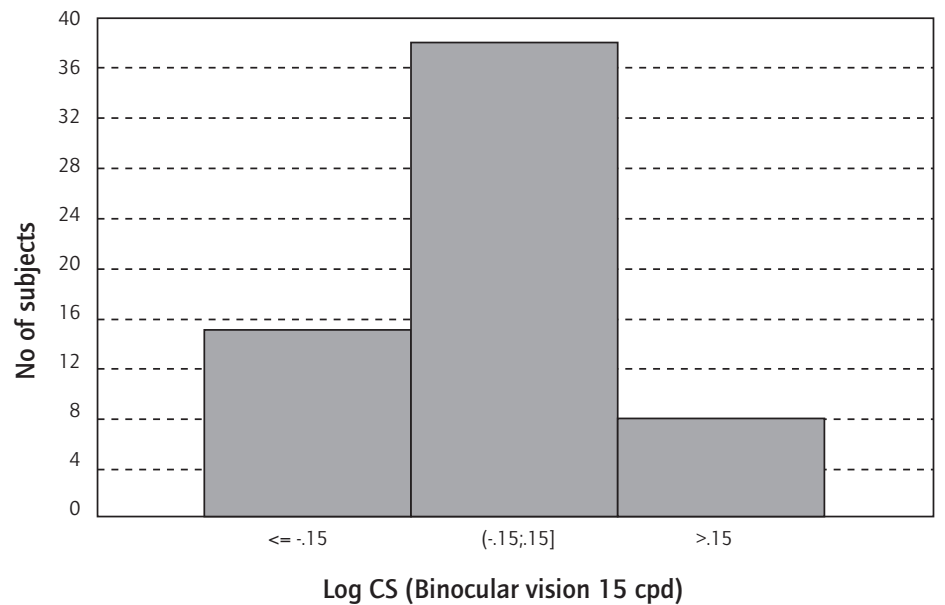

Figure 7. Distribution of average CS difference between corrected and aberrated lenses for binocular vision measured at $15 \mathrm{cpd}$.

conclusion because CS testing is less sensitive to dioptric effects at low frequencies, especially under binocular conditions. This could also be an explanation for the fact that the results at 6 cpd did not exhibit the same trend as the other conditions (Fig. 5).

The tendency of aberrated lenses to give better CS results is quite surprising. It appears that the previous conclusions of Cox and Holden ${ }^{17}$ do not apply to our subject sample. For the myopic group, a possible explanation of our results can be considered if we assume the aver- 
age spherical aberration of the eye to be slightly positive. ${ }^{22}$ According to this hypothesis, the negative aberration of a standard myopic lens put on the eye may reduce the aberration of the whole system and lead to better performance than a corrected lens. If we apply the same reasoning to the hyperopic group, we find that the positive aberration of standard hyperopic lenses should increase the total aberration. This is not consistent with our results. In fact, the published measurements of ocular spherical aberration involved few patients, and the generalization of the average positive aberration to the highly ametropic population has to be verified. High myopes and high hyperopes might belong to special groups with respect to ocular aberrations. This possibility is also suggested by the aberroscope measurements in myopic eyes reported by Collins et al.23

Yet, one must realize that the average differences observed in our study are inferior to the sensitivity decrease of approximately $0.15 \mathrm{LogCS}$, which would roughly be induced by 0.25 $\mathrm{D}$ of dioptric blur at $15 \mathrm{cpd} .{ }^{24}$ The smallness of these average effects could be attributed to lens flexure which, according to $\mathrm{Cox}^{4}$, may reduce the in vivo spherical aberration of soft contact lenses.

However, if we consider individual results, we can estimate that clinically significant differences $(>0.15$ or $<-0.15 \log C S$ at $15 \mathrm{cpd}$ ) between the lenses are observed in approximately one-third of the population (see histogram in Fig. 7). This rate suggests that an individual optimization of the lens aberration could result in an improvement of visual performance in part of the highly ametropic population. This possibility could be explored by trying lenses with various spherical aberration levels in such ametropes.

Also, pupil size is an important parameter in longitudinal spherical aberration. In the present study, we were interested to know about the systematic compensation of spherical aberration in this group of high ametropes, and used the relatively large natural pupil (under low room luminance condition) of the individual subject to compare the two lens types.

Individual eye aberration is a parameter that should be considered in order to theoretically calculate spherical aberration in the on eye situation. ${ }^{25}$ It would be of interest in further studies to measure the real shape of the cornea and that of the contact lens front surface in situ in order to compare theoretical modeling with in vivo individual results.

\subsubsection{Conclusion}

Our study suggests that there is no obvious clinical interest in correcting the longitudinal spherical aberration of soft contact lenses for high ametropes in a systematic way.

However, the optimization of longitudinal spherical aberration in high power lenses may significantly improve the CS of individuals. An object for further research could be to find discriminant factors for such improvements. Also, it would be of interest to investigate the modification that contact lenses with various aspheric front surfaces have on in vivo spherical aberration of the high ametropic eye, compared to spectacle lens correction.

\section{Acknowledgments}

We thank M. Salgues, D. Renard, and H. Molenaar for their experimental help; Dr. G.L. 
van der Heijde, Free University Amsterdam and Dr. F. Riemslag, Institute of Ophthalmic Research, the Netherlands for the use of the VSG; also we thank D. Baude of Essilor International $\mathrm{R} \& \mathrm{D}$ for useful discussions in preparing this paper.

\section{References}

1. Westheimer G. Aberrations of contact lenses. Am J Optom Arch Am Acad Optom 1961;38:445-8.

2. Campbell CE. The effect of spherical aberrations of contact lenses to the wearer. Am J Optom Physiol Opt 1981;58:212-7.

3. Bauer GT. Longitudinal spherical aberration of modern ophthalmic lenses and its effect on visual acuity. Appl Opt 1980;19:2226-34.

4. Cox I. Theoretical calculation of the longitudinal spherical aberration of rigid and soft contact lenses. Optom Vis Sci 1990;67:277-82.

5. Atchinson DA. Aberrations associated with rigid contact lenses. J Opt Soc Am 1995;12:2267-73.

6. Bauer GT, Lechner HB. Measurement of longitudinal spherical aberration of soft contact lenses. Optom Lett 1979;4:224-6.

7. Campbell MCW, Charman WN, Voisin L and Cui C. Psychophysical measurement of the optical quality of varifocal contact lenses. O.S.A. Technical Digest Series 3 1993:12-15.

8. Millodot M. Variation of visual acuity with soft contact lenses: as a function of luminance. Am J Optom Physiol Opt 1975;52:541-4.

9. Woo GC, Sivak JG. The effect of hard and soft contact lenses (Soflens) on the spherical aberration of the human eye. Am J Optom Physiol Opt 1976;53:459-63.

10. McGrath C, Morrison JD. The effects of age on spatial frequency perception in human subjects. Quart J Exp Physiol 1981;66:253-61.

11. Gilchrist I, McIver C. Fechner's paradox in binocular contrast sensitivity. Vision Res 1985;25:609-13.

12. Loshin DS, Loshin MS, Comer G. Binocular summation with monovision contact lens correction for presbyopia. ICLC 1982;9:161-5.

13. Bosse JC. Use of contrast sensitivity testing for contact lens fitting and management. Contact Lens J 1992;18;3:75-8.

14. Morrison JD. Contrast sensitivity: Methods and applications. Contact Lens J 1990;16;2:29-34.

15. Campbell FW, Green DG. Monocular versus binocular visual acuity. Nature 1965;208:191-2.

16. Eldred KB, Jose RT. Binocular contrast sensitivity testing for low vision patients. J Am Optom Assoc 1991;62:766-9.

17. Cox I, Holden BA. Soft contact lens-induced longitudinal spherical aberration and its effect on contrast sensitivity. Optom Vis Sci 1990;67:679-83.

18. Collins MJ, Brown B, Atchinson DA, Newman SD. Tolerance to spherical aberration induced by rigid contact lenses. Ophthal Physiol Opt 1992;12:24-8.

19. Corwin TR, Kintz RT, Beaty WJ. Computer-aided estimation of psychophysical thresholds by Wetherill tracking. Behav Res Methods Instrum Comput 1979;11:526-8.

20. Baude D. Caracterisation, optimisation et realisation de composants optiques multifocaux diffractifs et refractifs. PhD dissertation. Université de Paris-Sud, France, 1990.

21. Ginsburg AP, Evans DW, Cannon MW Jr, Owsley C, Mulvanny P. Large sample norms for contrast sensitivity. Am J Optom Physiol Opt 1984;16:80-4. 
22. Charman WN, Jennings JA. The optical quality of the monochromatic retinal image as a function of focus. Br J Physiol Opt 1976;31:119-34.

23. Collins MJ, Wildsoet CF, Atchinson DA. Monochromatic aberrations and myopia. Vision Res 1995;35:1157-63.

24. Herse PR, Bedell HE. Contrast sensitivity for letter and grating targets under various stimulus conditions. Optom Vis Sci 1989;66:774-81.

25. Hammer RM, Holden BA. Spherical aberration of aspheric contact lenses on eye. Optom Vis Sci 1994;71:522-28. 


\subsection{Feasibility of Correcting severe Optical Aberrations of the Eye}

Simulated Optical Performance of Custom Wave-Front Soft Contact Lenses for Keratoconus

J. de Brabander ${ }^{1}$, N. Chateau ${ }^{2}$, P. Artal ${ }^{3}$, G. Marin ${ }^{2}$, N. Lopez-Gil ${ }^{3}$, E. van der Worp ${ }^{1}$ and A. Benito ${ }^{3}$.

From:

1 Eye Research Institute Maastricht, Department of Ophthalmology Academic Hospital, Maastricht University, the Netherlands.

2 Ocular Sciences (formerly Essilor CL division), R\&D International, Créteil, France.

3 Laboratoria de Optica, Department of Physics, University of Murcia, Spain.

Submitted. 
Abstract

Introduction. Outstanding improvements of vision can theoretically be expected using contact lenses that correct the monochromatic aberrations of the eye. Imperfections in such correction inherent to contact lenses are: lens flexure, translation, rotation and tear layer effects. The effects of pupil size and accommodation may cause further difficulties.

Purpose. The purpose of this study was to evaluate if non-axisymmetric soft contact lenses could efficiently compensate for higher-order aberrations as induced by keratoconus and to what extent rotation and translation of the lens would degrade this perfect correction. Methods. Height topography data of nine moderate to severe keratoconus corneas were obtained using the Maastricht Shape Topographer (MST). Three-dimensional ray-tracing was applied to each elevation topography in order to calculate aberrations in the form of a phase error mapping. The effect of a non-axisymmetric soft contact lens tailored to the corneal aberrations was simulated by adding an opposite phase error mapping that would theoretically compensate all corneal induced optical aberrations of the keratoconus eyes. Translation ( 0.1 to $1.0 \mathrm{~mm}$ ) and rotation (1 to 10 degrees) mismatches were introduced. The Modulation Transfer Function (MTF) of each eye with each displaced correction and with various pupil sizes was deduced from the residual phase error mapping. A single performance criterion (mtfA) was calculated as the area under of the MTF over a limited spatial frequency range. Finally, the ratio ( $\mathrm{RmtfA}$ ) of corrected mtfA over uncorrected mtfA provided an estimate of the global enhancement in contrast sensitivity with the customized lens.

Results. The contrast improvement ratios RmtfA with perfectly located lenses were between 6.5 and 200. For small translation errors $(0.25 \mathrm{~mm})$, RmtfA ranged between 2 and 7 . The largest lens translation tested $(1 \mathrm{~mm})$ often resulted in poorer performance than without correction $(\mathrm{RmtfA}<1)$. More than three-fold improvements were achieved with any of the angular errors experimented. RmtfA values showed relatively little variations for pupil diameters between 3 and $6 \mathrm{~mm}$.

Conclusions. Three-dimensional aberration-customized soft contact lenses may drastically improve visual performance in patients with keratoconus. However, such lenses should be well positioned on the cornea. In particular translation errors should not exceed $0.5 \mathrm{~mm}$. Angular errors appeared to be less critical.

\section{Key Words:}

Eye, optical aberrations, keratoconus, soft contact lenses, custom refractive correction 


\subsubsection{Introduction}

Various techniques have been developed to measure the optical aberrations of the eye. ${ }^{1-4}$ These techniques can be subjective, objective or of psychophysical nature and all aim in general terms to determine the ability of the eye to focus images on the retina, often characterized in terms of optical transfer function (OTF). For most optical systems it is not difficult to obtain a complete description of the OTF, including, characteristics on transfer of modulation (MTF), phase (PTF), and the ability of the system to image point sources (point spread function, PSF), or lines (line spread function, LSF) at any wavelength and any angle of incidence. ${ }^{5}$ Furthermore, in optical systems the analysis can usually be made from both sides of the system and also test objects, sensors and apertures can be manipulated to desire.

Measurements on the eye are restricted in many ways. Most important is that the eye can only be assessed from one side and the in-vivo sensor is the retina connected to the brain. This means that one should either test subjectively, which incorporates the neural transfer function (NTF) in the system, or analyze the light that returns out of the eye after reflection on the retina (so called double-pass imaging) which introduces several uncertain factors. ${ }^{6}$ Also, the eye is not a stable optical system because both the optical power by accommodation and the aperture by pupil dynamics do vary with circumstances or environment. An other major complication in measuring the aberrations of the eye is the fact that it is a physiological system which expresses in variation of chromatic aberration with age ${ }^{7}$, hydration and condition of the tissues ${ }^{8}$ and disturbance of the tear film. ${ }^{9}$

With the above, it not surprising that, if normal eyes are considered as an optical system, it has been shown that even monochromatic aberrations vary substantially in order and magnitude among individuals. ${ }^{1,10}$ Although most authors found similar trends in the influences of refractive error ${ }^{11}$, accommodation ${ }^{12,13}$, and pupil size ${ }^{14,15}$ on monochromatic eye aberrations, they typically also indicate that these trends are not valid for all patients if subjectively assessed. These findings make it difficult to relate principles that have proven to work in the technical design of optical systems to a flexible biological system as is represented by the eye-brain complex. A typical example of the complexity of the monochromatic aberration effects on vision is the application of multifocal contact lenses. ${ }^{16,17}$

Despite the above, it is clear that the corneal shape ${ }^{18}$ (actually the tear film on it) represents by its interface with air the main refractive surface of the eye and therefor is largely responsible for its optical aberrations. ${ }^{19,20}$ The extreme deformation of corneal shape as is present in keratoconus degrades the retinal image to a degree that can make normal visual functioning impossible. Optical correction of the keratoconus eye with spectacle lenses is difficult because this mode is limited to correct only for the lower order optical aberrations of defocus and regular astigmatism. Therefore, rigid scleral and corneal contact lenses are widely used to correct the keratoconus eye. ${ }^{21,22}$ The principle behind these aids is that the irregular corneal surface is replaced by the regular anterior surface of the contact lens and the tear layer between cornea and posterior lens surface compensates the aberrations. However, usually the front surface of the corrective rigid contact lens is spherical. It has in normal eyes been demonstrated that such a surface would induce spherical aberration 
rather than correct it. ${ }^{19,23-26}$ Furthermore, the movement of a rigid lens on the irregular shaped cornea in advanced cases of keratoconus produces asymmetric refractive surfaces and consequently coma-like aberrations. ${ }^{27-30}$

The use of regular soft contact lenses on keratoconus eyes, even if they include correction for astigmatism (toric lenses), is not very successful because the flexibility of the lens makes it deform to match the irregular cornea. ${ }^{31}$ Even if this would not be the case regular toric lenses only compensate the lower order aberrations.

Attempts have been made to combine the advantages of soft and rigid contact lenses in one lens ${ }^{32}$ or with a system known as "piggyback"22,33,34 in which a rigid lens rides on a soft lens in situ. Although these modes can be successful in terms of comfort and visual correction of lower order aberrations, the problem of correcting the severe higher-order aberrations in cases like keratoconus remains.

Outstanding improvements of vision can theoretically be expected using contact lenses with custom three-dimensional shapes that correct all optical aberrations of the eye. However, this is only possible assuming that the perfect correction is present under all circumstances. Rigid contact lenses bear the inherent problem of substantial movement and rotation whereas normal soft contact lenses would be imperfect due to lens flexure. The effects of pupil size and accommodation on ocular aberration may bring further difficulties. Although it has been found that systematic correction of spherical aberration of soft contact lenses is of no use, it seems very well possible to correct spherical aberration individually. 35

Since severe optical aberrations are present in keratoconus it is of interest to study to what extent visual function could be improved using custom wave-front non-axisymmetric soft contact lenses. Studying the theoretical effects of translation and rotation of these aberration-customized soft contact lenses would give some insight in the practical feasibility of such corrective systems.

\subsubsection{Purpose}

The purpose of this study was to theoretically evaluate if non-axisymmetric soft contact lenses could efficiently compensate for higher-order aberrations as induced in keratoconus and to what extent rotation and translation of the lens would degrade this perfect correction.

\subsubsection{Methods}

Our methodology in this study involved 6 successive steps as follows (examples of the methodology in each step are given in Figures 1 to 3).

Step 1. The corneas of nine patients with keratoconus were assessed using the Maastricht Shape Topographer (MST), a system based on Fourier profilometry. ${ }^{36}$ The outcome of the measurement are $\mathrm{x}, \mathrm{y}$, z values of the height topography that can be presented as a color 


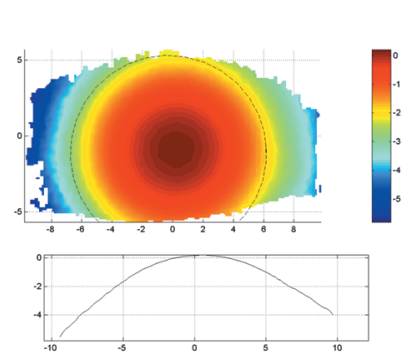

UNCORRECTED

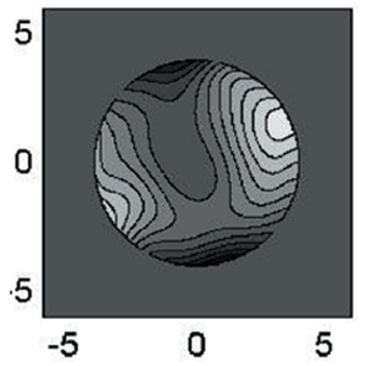

LENS ROTATION $2^{\circ}$

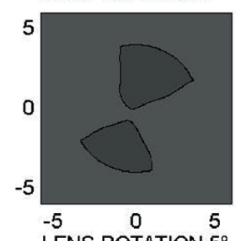

$\begin{array}{lcc}-5 & 0 & 5 \\ \text { LENS ROTATION } & 5\end{array}$
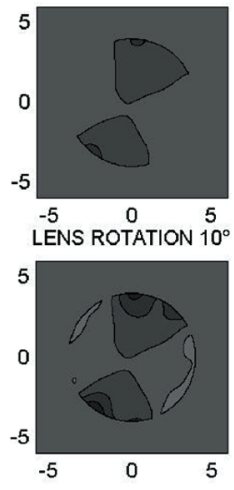

LENS TRANSLATION $0.2 \mathrm{~mm}$

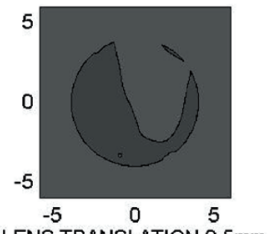

LENS TRANSLATION $\stackrel{5}{0.5 \mathrm{~mm}}$

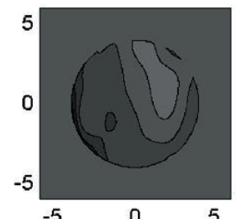

$\begin{array}{ll}-5 & 0 \\ \text { LENS TRANSLATION } & 5 \\ 1.0 \mathrm{~mm}\end{array}$

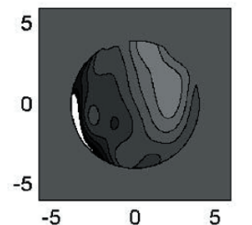

Figure 1. Example of steps 1 to 3 in computing wave-fronts from corneal topography with effects of rotation and translation. From the corneal height topography (top left, color coded map and cross section) the uncorrected phase error map (bottom left) is obtained. The influences on a perfect correction of the phase error map are shown for various degrees of rotation (middle column) and translation (right column).

coded height map or as a cross section profile (left top Fig.1).

Step 2. With the assumption that monochromatic aberrations were entirely due to the anterior shape of the cornea ${ }^{19,20}$ three-dimensional ray-tracing was applied to each height topography map in order to calculate the aberrations in the form of a phase error mapping (left bottom Fig. 1).

Step 3. The effect of a non-axisymmetric soft contact lens tailored to the corneal aberrations was simulated by adding an opposite phase error mapping. This would account for a perfect static correction of the optical aberrations of the keratoconus eyes. In this case the total eye + lens phase error would be constant (not shown). In order to simulate the real situation on the eye a rotational (2, 5 and 10 degrees, clockwise and counter clockwise) or a translation $(0.2,0.5$ and $1.0 \mathrm{~mm}$, in $\mathrm{X}$ and $\mathrm{Y}$ direction) mismatch of the surfaces was introduced. These errors were intended to account for the usual imprecision in positioning of a soft lens on the cornea due to movement and rotation with blinking. These factors are physiologically important in order to ensure the circulation of tears between the cornea and the contact lens. Examples of the resulting phase error maps with the introduced rotations or translations are shown in Fig. 1, middle column and right column, respectively.

Step 4. The modulation transfer function (MTF) of each eye with each displaced correction 

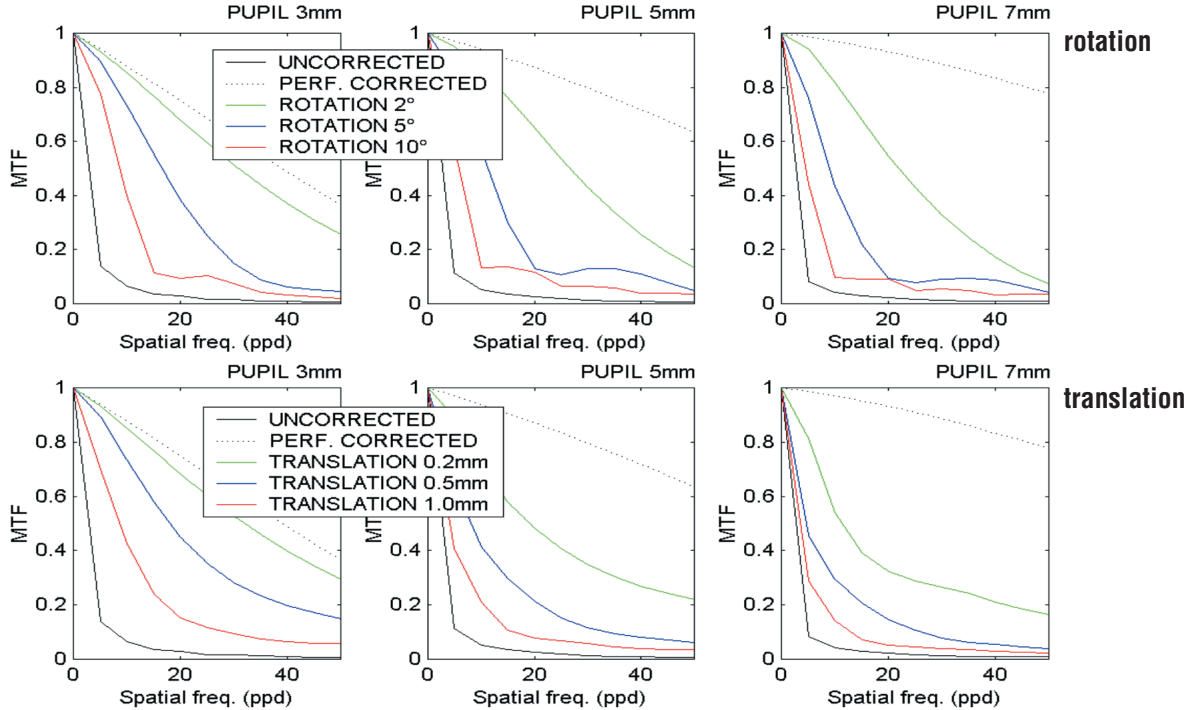

Figure 2. Example of steps 4 and 5 incorporating the computation of MTFs with induced rotation (top) and translation (bottom) for pupil sizes of $3 \mathrm{~mm}$ (left column), $5 \mathrm{~mm}$ (middle column) and $7 \mathrm{~mm}$ (right column). In each graph the MTF is drawn for the situation without correction, with perfect correction and with various amount of rotation or translation.
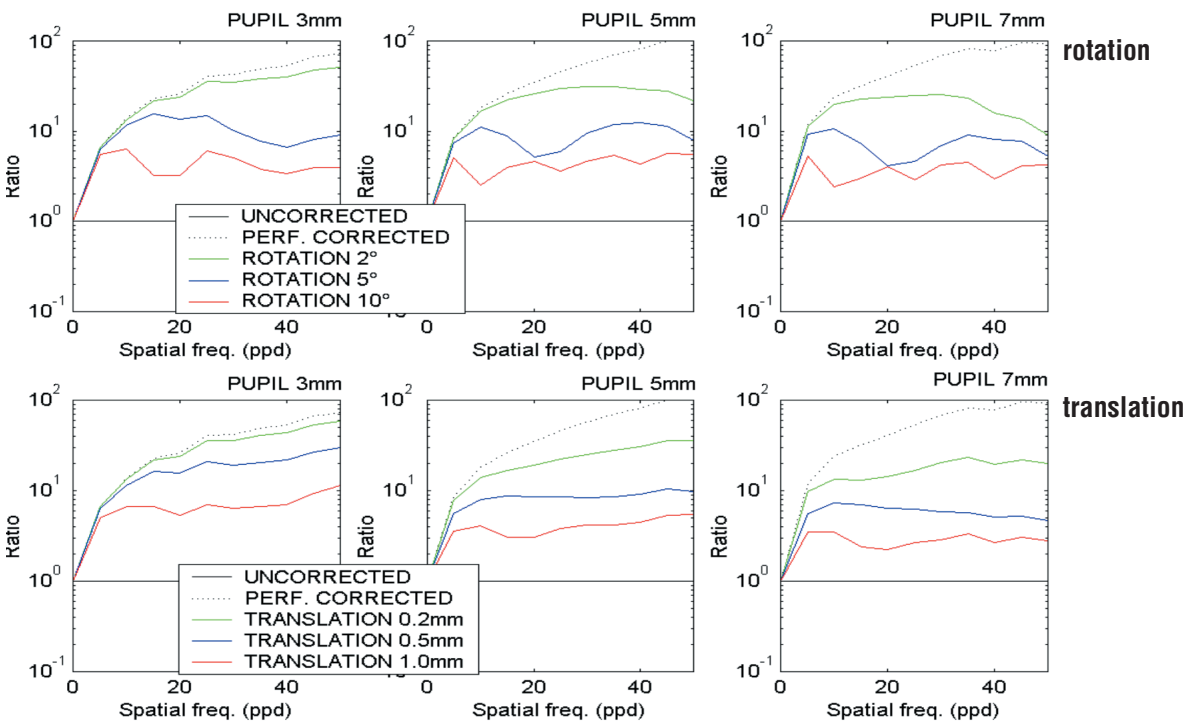

Figure 3. Example of step 6 showing the computed contrast improvement ratios for rotation effects (top), translation effects (bottom) and for pupil sizes of $3 \mathrm{~mm}$ (left column), $5 \mathrm{~mm}$ (middle column) and $7 \mathrm{~mm}$ (right column). In each graph the contrast improvement ratio for a spatial frequency range of 0 to 50 periods per degree (ppd) are given for the situation without correction, with perfect correction and with various amount of rotation or translation. 
was deduced from the residual phase error mapping. The MTFs were calculated for a wavelength of $550 \mathrm{~nm}$ incorporating the Stiles-Crawford effect. ${ }^{37}$ The same MTF calculations were repeated for pupil diameters of 3, 5 and $7 \mathrm{~mm}$. Figure 2 shows an example of the resulting data (one eye) in which the MTFs for uncorrected, perfectly corrected and rotations (top) or translations (bottom) are displayed in one graph per assumed pupil diameter.

Step 5. A single performance criterion (named $\mathrm{mtfA}$ ), was calculated as the area under the MTF over a limited spatial frequency range ( 5 to 15 periods per degree, ppd).

Step 6. Finally, the ratio (named RmtfA) of corrected mtfA over uncorrected mtfA was computed using the integrals of MTF's between 5 and 15 ppd. This procedure is known as the "normalized area evaluation of image quality" 5 and provides an estimate of the global enhancement in contrast sensitivity with the customized lens. Similar to Fig. 2, the results of computation of the RmtfA on one eye in the various situations are as an example presented in Fig. 3.

\subsubsection{Results}

The final results of the calculated RmtfA on all 9 cases and an average pupil diameter of 4.5 $\mathrm{mm}$ are for rotations given in Table 1 and for translations in Table 2. The cases are listed in relation to the severity of the keratoconus according to the classification of Amsler ${ }^{38}$ from moderate (case 1) to severe (case 9). For comparison, the second column in each table presents the RmtfA assuming a perfect centered lens (perfect position). Also, at the bottom of Tables 1 and 2 the "Total" numbers represent the (minimum, median and maximum) RmtfA relative to the uncorrected situation for all eyes and for pupil diameters ranging from $6.0 \mathrm{~mm}$ (Min) to $3.0 \mathrm{~mm}$ (Max).

The contrast improvement ratios RmtfA with perfectly located lenses and for pupil sizes from 6.0 to $3.0 \mathrm{~mm}$ ranged between 6.5 to 195 with a median of 39.6 (Tables 1 and 2, bottom left).

The RmtfA for introduced rotations clockwise and counter clockwise and from 2.5 to 10.0 degrees show a gradually decline in RmtfA with almost no difference between clockwise and counter clockwise rotations (Table 1, bottom right). Even the worse case of 10 degrees clockwise rotation and a largest pupil diameter $(6.0 \mathrm{~mm})$ would still theoretically enhance contrast sensitivity in a ratio of 2.8 .

The same analysis can be made from Table 2 (bottom right) for translations. Here it is shown in general that (although there is more variation than with rotations) the differences between displacements in $\mathrm{X}$ and $\mathrm{Y}$ directions are small. As with rotations the RmtfA declines gradually with the increase of the amount of translation. Contrary to the effects of rotations, for translations above $0.5 \mathrm{~mm}$ the minimal reached RmtfA drops below one, meaning theoretically that contrast sensitivity with such a lens in place under extreme conditions (large decentration and large pupil) would be worse than uncorrected. However, the median and the maximum RmtfA for translations still show values around or above one. The largest acceptable translation error for which a benefit in RmtfA is still maintained revealed to be $0.5 \mathrm{~mm}$. 
Table 1. RmtfA results for rotation.

Numbers in the table account for an average pupil diameter of $4.5 \mathrm{~mm}$ and are relative to RmtfA 1.0 in the uncorrected situation. Perfect position means zero rotation (perfect positioning of the lens). The RmtfA values are for each case given for 2.5, 5.0, 7.5 and 10 degrees for rotation clockwise and counterclockwise (Italic numbers). The Total numbers (bottom lines) represent the (minimum, median and maximum) RmtfA relative to the uncorrected situation, for pupil diameters ranging from 6.0 to $3.0 \mathrm{~mm}$.

\begin{tabular}{|c|c|c|c|c|c|}
\hline \multirow[t]{2}{*}{ Case } & \multirow{2}{*}{$\begin{array}{l}\text { Perfect } \\
\text { Position }\end{array}$} & \multicolumn{3}{|c|}{ Rotation clockwise and counterclockwise } & \multirow[b]{2}{*}{10.0} \\
\hline & & 2.5 & 5.0 & 7.5 & \\
\hline \multirow[t]{2}{*}{1} & 10.08 & 8.38 & 5.87 & 4.07 & 2.96 \\
\hline & & 8.38 & 5.87 & 4.06 & 2.95 \\
\hline \multirow[t]{2}{*}{2} & 13.05 & 10.03 & 6.51 & 4.56 & 3.60 \\
\hline & & 10.02 & 6.49 & 4.53 & 3.57 \\
\hline \multirow[t]{2}{*}{3} & 56.02 & 23.03 & 12.35 & 9.10 & 6.90 \\
\hline & & 23.06 & 12.40 & 9.16 & 6.92 \\
\hline \multirow[t]{2}{*}{4} & 65.80 & 25.55 & 9.40 & 6.44 & 5.32 \\
\hline & & 25.54 & 9.39 & 6.44 & 5.28 \\
\hline \multirow[t]{2}{*}{5} & 73.87 & 22.42 & 7.04 & 4.50 & 3.57 \\
\hline & & 22.34 & 7.02 & 4.50 & 3.58 \\
\hline \multirow[t]{2}{*}{6} & 29.63 & 20.83 & 11.40 & 6.79 & 4.99 \\
\hline & & 20.83 & 11.42 & 6.81 & 5.01 \\
\hline \multirow[t]{2}{*}{7} & 20.07 & 12.87 & 6.60 & 4.35 & 3.32 \\
\hline & & 12.86 & 6.56 & 4.31 & 3.28 \\
\hline \multirow[t]{2}{*}{8} & 91.06 & 21.58 & 8.24 & 4.54 & 3.19 \\
\hline & & 21.56 & 8.25 & 4.50 & 3.20 \\
\hline \multirow[t]{2}{*}{9} & 194.65 & 31.19 & 14.34 & 8.82 & 6.24 \\
\hline & & 31.21 & 14.18 & 8.68 & 6.26 \\
\hline \multicolumn{6}{|l|}{ Total } \\
\hline \multirow[t]{2}{*}{ Min } & 6.47 & 6.15 & 5.32 & 3.99 & 2.83 \\
\hline & & 6.15 & 5.32 & 4.00 & 2.85 \\
\hline \multirow[t]{2}{*}{ Median } & 39.58 & 20.83 & 8.51 & 5.20 & 4.13 \\
\hline & & 20.83 & 8.52 & 5.19 & 4.10 \\
\hline \multirow[t]{2}{*}{ Max } & 194.65 & 38.02 & 15.78 & 11.06 & 8.93 \\
\hline & & 37.99 & 15.85 & 17.14 & 9.01 \\
\hline
\end{tabular}




\begin{tabular}{|c|c|c|c|c|c|c|c|c|c|}
\hline \multirow{2}{*}{ Case } & \multirow{2}{*}{$\begin{array}{l}\text { Perfect } \\
\text { Position }\end{array}$} & \multicolumn{4}{|c|}{ Translation $+\mathrm{X}$ and $-\mathrm{X}$} & \multicolumn{4}{|c|}{ Translation $+Y$ and $-Y$} \\
\hline & & 0.25 & 0.50 & 0.75 & 1.00 & 0.25 & 0.50 & 0.75 & 1.00 \\
\hline \multirow[t]{2}{*}{1} & 10.08 & 3.60 & 1.75 & 1.16 & 0.87 & 3.69 & 1.64 & 1.02 & 0.79 \\
\hline & & 3.64 & 1.85 & 1.26 & 0.97 & 3.71 & 1.70 & 1.17 & 0.93 \\
\hline \multirow[t]{2}{*}{2} & 13.05 & 3.40 & 1.54 & 1.40 & 1.26 & 3.22 & 1.59 & 1.05 & 0.76 \\
\hline & & 3.29 & 1.25 & 0.91 & 0.74 & 3.50 & 1.97 & 1.45 & 1.09 \\
\hline \multirow[t]{2}{*}{3} & 56.02 & 5.95 & 2.79 & 1.91 & 1.52 & 4.60 & 2.36 & 1.57 & 1.19 \\
\hline & & 6.23 & 2.93 & 1.95 & 1.61 & 4.44 & 2.28 & 1.57 & 1.20 \\
\hline \multirow[t]{2}{*}{4} & 65.80 & 7.35 & 4.08 & 2.75 & 2.19 & 5.75 & 2.96 & 2.23 & 1.76 \\
\hline & & 7.33 & 4.15 & 2.82 & 2.21 & 5.65 & 2.74 & 2.15 & 1.73 \\
\hline \multirow[t]{2}{*}{5} & 73.87 & 4.91 & 2.73 & 1.87 & 1.44 & 3.83 & 2.15 & 1.42 & 1.05 \\
\hline & & 5.08 & 2.63 & 1.74 & 1.42 & 4.05 & 2.42 & 1.86 & 1.71 \\
\hline \multirow[t]{2}{*}{6} & 29.63 & 3.33 & 1.60 & 1.02 & 0.81 & 3.85 & 1.87 & 1.27 & 0.93 \\
\hline & & 3.20 & 1.60 & 1.02 & 0.78 & 3.76 & 1.74 & 1.18 & 0.88 \\
\hline \multirow[t]{2}{*}{7} & 20.07 & 2.89 & 1.28 & 0.85 & 0.64 & 2.58 & 1.41 & 1.10 & 0.86 \\
\hline & & 3.02 & 1.36 & 1.01 & 0.82 & 2.63 & 1.43 & 1.05 & 0.80 \\
\hline \multirow[t]{2}{*}{8} & 91.06 & 3.56 & 1.87 & 1.32 & 1.00 & 3.48 & 1.65 & 1.08 & 0.95 \\
\hline & & 3.57 & 1.82 & 1.38 & 1.07 & 3.38 & 1.68 & 1.10 & 0.87 \\
\hline \multirow[t]{2}{*}{9} & 194.65 & 3.91 & 1.98 & 1.47 & 1.33 & 5.12 & 2.67 & 2.17 & 1.65 \\
\hline & & 4.12 & 2.17 & 1.62 & 1.21 & 3.71 & 1.59 & 1.22 & 1.09 \\
\hline \multicolumn{10}{|l|}{ Total } \\
\hline \multirow[t]{2}{*}{ Min } & 6.47 & 1.92 & 1.02 & 0.74 & 0.57 & 2.18 & 1.18 & 0.89 & 0.17 \\
\hline & & 2.19 & 1.25 & 0.91 & 0.72 & 2.23 & 1.24 & 0.88 & 0.67 \\
\hline \multirow[t]{2}{*}{ Median } & 39.58 & 3.73 & 1.87 & 1.32 & 1.03 & 3.85 & 1.86 & 1.26 & 0.94 \\
\hline & & 3.83 & 1.85 & 1.29 & 1.06 & 3.89 & 1.90 & 1.25 & 1.04 \\
\hline \multirow[t]{2}{*}{ Max } & 194.65 & 7.35 & 4.08 & 2.75 & 2.19 & 5.75 & 2.96 & 2.23 & 1.76 \\
\hline & & 7.33 & 4.15 & 2.82 & 2.21 & 5.65 & 2.99 & 2.16 & 1.73 \\
\hline
\end{tabular}

The same type of analyses on the data can be made for the upper parts of Tables 1 and 2 representing the results of RmtfA with an assumed average pupil of $4.5 \mathrm{~mm}$. From Table 1 (top right) it can be deduced that, with minor differences between the direction of rotation the gain in contrast sensitivity with $4.5 \mathrm{~mm}$ pupil size would be 3 -fold or more. From the data as given in Table 2 it is clear that translation affects the RmtfA more than rotation does. Variation in $\mathrm{X}$ or $\mathrm{Y}$ direction is small (with some exceptions). For the $4.5 \mathrm{~mm}$ pupil it seems that $0.75 \mathrm{~mm}$ is a critical translation value in order to keep RmtfA above one. In general, 
with a larger pupil diameter $(6.0 \mathrm{~mm})$ translation should not exceed $0.5 \mathrm{~mm}$ in order to still benefit from the optical correction that the customized soft lens would give including the most severe keratoconus of this study.

\subsubsection{Discussion}

In this study concerning the theoretical enhancements that customized non-axisymmetrical soft contact lenses could offer in visual functioning of severely aberrated eyes as in keratoconus, we found little variations in $(\mathrm{RmtfA})$ results between the directions of rotation (clockwise and counter clockwise) or translation (X or Y direction) of such lenses simulated on the eye.

However, the magnitude of translation seems to be much more critical than that of rotation. We found that translations in order to benefit RmtfA, have a critical value of $0.5 \mathrm{~mm}$ for larger pupils and $0.75 \mathrm{~mm}$ for average $4.5 \mathrm{~mm}$ pupils. This is just around the boarder of what has been established as a clinically efficient movement of soft contact lenses to avoid compromising tear circulation. ${ }^{39}$

If compared to the much larger movements as are present in the application of rigid contact lenses, our results indicate that from a standpoint of correcting optical aberrations, customized non-axisymmetric soft contact lenses would be superior to rigid lenses. However, it remains a question how in reality the minor movement of non-axisymmetric soft lenses with possible changes of their anterior shapes due to flexure ${ }^{31}$ would balance the more pronounced movement but the more solid anterior surfaces of rigid lenses in optical performance.

Taken into consideration the criterion of Maréchal ${ }^{40}$, optics in normal young eyes even with $7 \mathrm{D}$ of myopia, accommodation up to $3 \mathrm{D}^{11}$ or in the presence of front surface aspheric soft contact lenses ${ }^{41}$ operate nearly diffraction limited at a pupil size of around $3 \mathrm{~mm}$. In our calculations assuming perfectly situated non-axisymmetric aberration customized soft contact lenses a nearly diffraction limited correction could theoretically be obtained for pupil sizes up to $7 \mathrm{~mm}$ (Fig. 2). This likely means that the retina becomes the limiting element of the entire optical system of the eye under the corrective circumstances we have computed. Due to the increased Strehl ratio 5 , which means a more efficient distribution of light energy in the image, contrast vision may even further be improved with such lenses in situ. On the other hand, our calculations were solely based on corneal topographic data and pupil size assuming a perfect centration of the pupil in relation to the topography. It has been shown that even a small pupil diameter of $1.5 \mathrm{~mm}$ can be critical in the optical system concerning pupil centration. ${ }^{3}$ This could in real application of the non-axisymmetric soft contact lenses as subject to our theoretical calculations degrade the final image quality.

We have found from our theoretical calculations that a perfect correction using non-axisymmetric soft contact lenses in keratoconus eyes can be achieved. However, the improvement in image quality varies substantially among cases and in particular with the critical value of physiologically demanded translation respecting tear film exchange. Assuming the in situ application of non-axisymmetric soft contact lenses designed to theoretically compensate all ocular aberration of the eye, the interesting question is whether the NTF of the eye-brain complex in keratoconus eyes would be able to compensate (average) the variation in optical performance 
related to the temporary variation due to lens movement or would be confused by it. There seems to be some similarity with the phenomena as encountered with the application of varifocal soft contact lenses ${ }^{17}$ in which also a combination between the Stiles Crawford effect, the Strehl ratio, pupil size, behavior of the lens and the individual NTF seems to play an important role in the visual satisfaction finally reported by the individual patient.

The Amsler ${ }^{38}$ classification on which the cases in our study were ranked in severity (1 to 9, in Tables 1 and 2) shows no relation with our data on RmtfA in the perfect position (with the exception of cases 1, 2 and 8,9). This means that central radius of curvature of the cornea and the distortion of keratometric mirrors are no adequate predictors of optical aberrations in keratoconus. This is in accordance with clinical practice and with studies done on the visual outcome with contact lenses to correct keratoconus which in relation to corneal parameters vary substantially among subjects. ${ }^{42-48}$

Recently, Guirao et al. ${ }^{49}$ computed the residual aberrations that appear as a result of translation or rotation on an otherwise ideal correction in ten normal eyes of which actual wave aberrations were obtained using a Shack-Hartmann sensor. In their study they only report on one keratoconus case as an example. This is due to the present limitations of the systems to measure severely aberrated eyes with the normally used Shack-Hartmann technique. For this reason we have computed the aberrations from corneal topographic data rather than make attempts to really measure it globaly in the eyes. Nevertheless, the results of the two studies are very much comparable. This means that the correction of optical aberrations in normal eyes and that of eyes suffering from keratoconus would theoretically lead to the same results.

In summary, three-dimensional custom wave-front soft contact lenses may drastically improve visual performance in patients with keratoconus. However, such lenses should be positioned on the cornea within more severe tolerances than conventional contact lenses. More than three-fold improvements were achieved with any of the angular errors experimented, meaning that angular errors appeared to be less critical.. Translation errors showed to be more critical and should ideally not exceed $0.5 \mathrm{~mm}$. From the data resulting from the present study it can be predicted that patients suffering from severe keratoconus would probably benefit from correction with customized non-rotational symmetric soft contact lenses given that the translation of the lenses can be limited to $0.5 \mathrm{~mm}$ and the retinal-brain system is able to adapt to the variations in presented optical images to the retina.

\section{Acknowledgement}

This study was part of the BRITE-EURAM project BE96-4608, Contact Lenses with Aspheric Asymmetric Surfaces (CLAAS) and supported by a grant from the European Commission. 


\section{References}

1. Howland B, Howland H. Subjective measurement of higher-order aberrations of the eye. Science 1976;193:580-82.

2. Charman WN. Wavefront aberration of the eye: a review. Optom Vis Sci 1990;68:574-83.

3. Artal P, Iglesias I, López-Gil N. Double-pass measurements of the retinal-images quality with unequal entrance and exit pupil sizes and the reversebility of the eye's optical system. J Opt Soc Am 1995;12:2358-66.

4. Moreno-Barriuso E, Marcos S, Navarro R, Burns SA. Comparing Laser Ray Tracing, the Spatially Resolved Refractometer, and the Hartmann-Shack Sensor to Measure the Ocular Wave Aberration. Optom Vis Sci 2001;78:152-56.

5. Smith WJ. Modern Optical Engineering. Chapter 11. New York, Mc Graw-Hill Book Company, 1966.

6. Losada M, Navarro R, Santamaria J. Relative contributions of Optical and Neural Limitations to Human Contrast Sensitivity at Diferent Luminance Levels. Vis Res 1993;33:2321-36.

7. Millodot M. The influence of age on the chromatic aberration of the eye. Albrecht Von Graefes Arch Klin Exp Ophthalmol 1976;198:235-43.

8. Cohen SR, Polse KA, Brand RJ, Mandell RB. Humidity effects on corneal hydration. Invest Ophthalmol Vis Sci 1990;31:1282-7.

9. Guillon M, Styles E, Guillon JP, Maissa C. Preocular tear film characteristics of nonwearers and soft contact lens wearers. Optom Vis Sci 1997;74:273-9.

10. Brink van den G. Mesurements of the geometrical aberrations of the eye. Vis Res 1962;2:233-44.

11. Collins M, Wildsoet C, Atchison D. Monochromatic Aberations and Myopia. Vis Res 1995;35:115763.

12. Ivanoff E. About the spherical aberration of the eye. J Opt Soc Am 1963;46:901-4.

13. Schober H, Munker H, Zolleis F. Variations of aberration with accommodation. Opt Acta 1968;15:4757.

14. Walsh G, Charman WN. Measurement of the axial wavefront aberration of the human eye. Ophthalmic and Physiological Optics 1985;5:23-31.

15. Artal P, Navarro R. Monochromatic modulation transfer function of the human eye for different pupil diameters: an analytical expression. J Opt Soc Am 1994;11:246-49.

16. Atchison DA, Thibos LN. Diffractive properties of the Diffrax bifocal contact lens. Ophthalmic Physiol Opt 1993;13:186-8.

17. Brabander de J, Chateau N, Molenaar H, Bouchard F. Presbyopia and Contact Lenses: A Literature Review and a Clinical Study. Contactologia 2000;22:99-108.

18. Mandell RB. The Enigma of the Corneal Contour. CLAO J 1992;18:267-73.

19. Campbell CE. The effect of spherical aberration of contact lens to the wearer. Am J Optom Physiol Opt 1981;58:212-7.

20. Seiler T, Reckmann W, Maloney RK. Effective spherical aberration of the conea as a quantitative descriptor in corneal topography. J Cat Refr Surg 1993;19:155-67.

21. Kok JH, Wagemans MA, Rosenbrand RM, von Mil C. Computer assistance in keratoconus lens design. CLAO J 1990;16:262-5.

22. Kok JH, van Mil C. Piggyback lenses in keratoconus. Cornea 1993;12:60-4.

23. Westheimer G. Aberrations of contact lenses. Am J Optom Arch Am Acad Optom 1961;38;8:445-8. 
24. Cox I. Theoretical calculation of the longitudinal spherical aberration of rigid and soft contact lenses. Optom Vis Sci 1990;67:277-82.

25. Hammer RM, Holden BA. Spherical aberration of aspheric contact lenses on eye. Optom Vis Sci 1994;71:522-8.

26. Atchison DA. Aberrations associated with rigid contact lenses. J Opt Soc Am A 1995;12:2267-73.

27. Oxenberg LD, Carney LG. Visual performance with aspheric rigid contact lenses. Optom Vis Sci 1989;66:818-21.

28. Carney LG. Is the quality of vision with contact lenses adequate? Not only adequate but often superior. Cornea 1990;9:S23-4.

29. Forst G. Comparison of the movement of contact lenses with spherical and aspherical back surfaces. Contact Lens Journal 1990;18:129-32.

30. Campbell MWC, Harrison EM, Simonet P. Psycho-physical measurement of the blur on the retina due to optical aberrations of the eye. Vis Res 1990;30:1587-1602.

31. Holden BA, Zantos SG. On the conformity of soft lenses to the shape of the cornea. Am J Optom Physiol Opt 1981;58:139-43.

32. Maguen E, Caroline P, Rosner IR, Macy JI, Nesburn AB. The use of the SoftPerm lens for the correction of irregular astigmatism. CLAO J 1992;18:173-6.

33. Massin M, Denis Morere A, Ninine G. [Keratoconus and contact lenses (author's transl)]. Klin Monatsbl Augenheilkd 1976;168:24-32.

34. Soni PS, Gerstman DR, Horner DG, Heath GG. The management of keratoconus using the corneal modeling system and a piggyback system of contact lenses. J Am Optom Assoc 1991;62:593-7.

35. Brabander de J, Chateau N, Bouchard F, Guidollet S. Contrast sensitivity with soft contact lenses compensated for spherical aberration in high ametropia. Optom Vis Sci 1998;75:37-43.

36. Jongsma FH, de Brabander J, Hendrikse F, Stultiens BA. Development of a wide field height eye topographer: validation on models of the anterior eye surface. Optom Vis Sci 1998;75:69-77.

37. Stiles WS, Crawford BH. The luminous efficientcy of rays entering the eye pupil at different points. Proceedings of the Royal Society of London in Biology 1933;112:428-50.

38. Amsler M. Early diagnosis and microsymptoms. Ophthalmologica 1965;149:438-46.

39. Terry RL, Schnider CM, Holden BA, Cornish R, Grant T, Sweeney D, La Hood D, Back A. CCLRU standards for success of daily and extended wear contact lenses. Optom Vis Sci 1993;70:234-43.

40. Born M, Wolf E. Principles of optics. Ed. 3; section 9. Oxford, Pergamon Press, 1975.

41. Cox I, Holden BA. Soft contact lens-induced longitudinal spherical aberration and its effect on contrast sensitivity. Optom Vis Sci 1990;67:679-83.

42. Carney LG. Contact lens correction of visual loss in keratoconus. Acta Ophthalmol Copenh 1982;60:795-802.

43. Zadnik K, Mutti DO. Contact lens fitting relation and visual acuity in keratoconus. Am J Optom Physiol Opt 1987;64:698-702.

44. Carney LG, Lembach RG. Management of keratoconus: comparative visual assessments. CLAO J 1991;17:52-8.

45. Wilson SE, Klyce SD. Advances in the analysis of corneal topography. Surv Ophthalmol 1991;35:26977.

46. Wicker D, Sanislo S, Green DG. Effect of contact lens correction of sine wave contrast sensitivity in keratoconus patients after penetrating keratoplasty. Optom Vis Sci 1992;69:342-6.

47. Edrington TB, Zadnik K, Barr JT. Keratoconus. Optom Clin 1995;4:65-73. 
48. Szczotka LB, Thomas J. Comparison of axial and instantaneous videokeratographic data in keratoconus and utility in contact lens curvature prediction. CLAO J 1998;24:22-8.

49. Guirao A, Williams DR, Cox IG. Effects of rotation and translation on the expected benefit of an ideal method to correct the eye's higher- order aberrations. J Opt Soc Am 2001;18:1003-15. 
Chapter 5

Measuring Techniques 


\subsection{Introduction to this chapter}

The advancements in designing and manufacturing contact lenses $(\mathrm{CLs})^{1,2}$ was one of the reasons to develop devices that measure more precisely the anterior shape of the eye. Many attempts were made to modify the original keratometer. ${ }^{3-7}$ Today eye topography by means of video keratography is an important aid in prescribing CLs. ${ }^{8,9}$ However, this instrumentation covers a limited area of the eye and has some inherent problems of principle. ${ }^{10}$ In this chapter we describe the development and the validation of a wide field height eye topographer, the Maastricht Shape Topographer (MST, section 5.2.1).

The corneal-scleral profile is in fitting soft and scleral CLs and monitoring pathological conditions of importance. ${ }^{11}$ However, with conventional devices these aspects are difficult to visualize or measure. A feature of the MST is that it is able to present a profile cross-section of the entire eye front surface including the limbal area. Therefore we conducted a feasibility study in which we compared the nasal and temporal limbal angles in normal and keratoconus eyes (5.2.2).

The size and position of the pupil is an important element in the optical system of the eye. ${ }^{12-}$ 15 In modern research and the possibility to more precisely design CLs, pupillometry has become an important issue. Pupillometry is also important in the design of CLs that would correct presbyopia and optical aberrations of the eye, as has been shown in chapter 4 of this thesis. We developed a special infrared pupillometer and used it in a study with a group of presbyopic patients (5.3).

In ophthalmic practice the use of fluorescein is well known. ${ }^{16,17}$ Relatively new is the use of fluorescein in shape measurements of the eye and to visualise superficial or deeper layers of the tissues adherent to the sclera. In an in vivo (5.4) study we looked at fluorescence phenomena in relation to excitation wavelength, type of fluorescein, substrate and layer thickness.

\section{References}

1. Bier N. The Contour Lens. Contact Lens Routine and Practice. Ed. 2;p193-206. London, Butterworths, 1957.

2. Hirst E. Fitting a true asperical contact lens. Contacto 1974:15-9.

3. Placido A. Novo instrumento de esploracao da cornea. Per d'Oftalmol Pract 1880;5:27-30.

4. Mandell R. Morphometry of the Human Cornea, thesis, Indiana University, 1962.

5. Mandell RB. Methods to measure the peripheral corneal curvature. J Am Optom Ass 1962:889-92.

6. Wilms K, Rabbetts R. Practical concepts of corneal topometry. Optician 1977;Sept 16:7-13.

7. Wilms K. Ueberlegungen zur Topometrie von Cornea und Kontaktlinsen. Neues Optik J 1980;15:569-71.

8. Gullstrand A. Photographisch-ophthalmometrische und klinische Untersuchungen uber die Hornhautrefraktion. 1896:28.

9. Klyce S, Wilson S. Methods of Analysis of Corneal Topography. Refract Corneal Surg 1989;5:368-371. 
10. Jongsma FHM, Brabander de J, Hendrikse F. Review and Classification of Corneal Topographers. Lasers Med Sci 1999;14:2-19.

11. Pullum KW. Eye impressions, production and fitting of scleral lenses and patient management. Contact Lenses. Ed. 3, edited by Phillips A, Stone J. London, Butterworths, 1989.

12. Borish, I.M. Pupil dependency of bifocal contact lenses. Am. J. Optom. Physiol. Opt. 1988;65:417423 .

13. De Groot, S.G. and Gebhard, J.W. Pupil size as determined by adapting luminance. J Opt Soc Am 1952;42:492-5.

14. Lowenstein, O. and Loewenfeld, I.E. The pupil. The Eye, Ed. 2 (Ed. Davson, H.), 1969; vol. 3: 255327. Academic Press, New York, USA.

15. Schäfer, R. and Weale, R. The influence of age and retinal illumination on the pupillary near reflex. Vision Res. 1970;10:179-191.

16. Maurice DM. The use of fluorescein in ophthalmological research. Invest Ophthalmol 1967;6(5):464477.

17. Passmore JW, King JH. Vital staining of conjunctiva and cornea. Arch Ophthalmol 1955;55:568-574. 


\subsection{Topography of the anterior Eye Surface}

\subsubsection{Development of a Wide Field Height Eye Topographer: Validation on Models of the Anterior Eye Surface}

Franciscus H.M. Jongsma ${ }^{1}$, John de Brabander ${ }^{1}$, Fred Hendrikse ${ }^{1}$ and Bertho A.T. Stultiens ${ }^{2}$.

From:

1 Eye Research Institute Maastricht, Department of Ophthalmology Academic Hospital, University Maastricht, the Netherlands.

2 Department of Ophthalmology, Århus University Hospital, Denmark.

Optom Vis Sci 1998;75;1:69-77. 
Abstract

Purpose. The aim of this research was to develop a corneal topographer that determines the shape of the entire anterior surface of an eye without assumptions, and with uniformly high precision in the center and periphery.

Methods. Based upon a double projection of two sine wave gratings and analysis of the distortion of the sine wave gratings due to the corneal-scleral shape, point-by-point measurements of surface elevation were obtained with a sample density equal to the pixel density of the CCD-detector. Using this principle, a prototype topographer, called the Maastricht Shape Topographer (MST) was developed. The accuracy and reproducibility of the instrument were evaluated using bispheric models of the anterior surface of the eye.

Results. The average accuracy of height measurements was $0.55 \mu \mathrm{m}$ in the $10-\mathrm{mm}$ central area and $22.50 \mu \mathrm{m}$ in the periphery (14 to $19 \mathrm{~mm}$ ). Reconstruction accuracy of the radius of curvature was $\pm 0.0155 \mathrm{~mm}( \pm 0.08 \mathrm{D})$ in the center and $\pm 0.0313 \mathrm{~mm}$ in the periphery (sclera). Average height reproducibility standard error was $0.0282 \mu \mathrm{m}$ in the center and $2.6156 \mu \mathrm{m}$ in the periphery.

Conclusions. With the MST, unambiguous shape measurements of the entire anterior surface of the eye are possible, with accuracy up to clinical accepted standards. The MST is able to measure height over a wide area of $20 \mathrm{~mm}$, with a $6 \mathrm{~mm}$ depth of field. The tested prototype of the device can be further improved by the use of custom-made optics in order to increase signal to noise ratio in the periphery of the image. This height topographer could offer a reliable method in cases where shape is of paramount importance, e.g., in (scleral) contact lens fitting and refractive surgery.

\section{Key words:}

Corneal topography, contact lens fitting, sclera, moiré, videokeratography. 


\subsubsection{Introduction}

Placido disc-based corneal topographers use the cornea as a convex mirror. The raw data in this type of topographer are highly sensitive for slope deviations. In Placido systems, the first Purkinje image, which is formed behind the cornea, is viewed for analysis. Historically, an assumption is made on the location and orientation of the image, and also on the shape that was actually measured. These assumptions, however, may not be applicable. Recently, some Placido systems have reduced the assumptions for identifying the corneal surface to the need of a continuous surface. However, the newer methods rely on relating adjacent measurements, which requires a dense and precise reflection pattern that cannot always be obtained. Because the shape of the cornea determines where the mires will reflect, the reflected image provides different coverage of different corneas, and does not consistently reveal structures outside the cornea.

A way to determine the real shape of the cornea directly, unambiguous and without assumptions, is to measure its local height. There are a variety of modalities to measure local height of objects in general. Most of them cannot be used in optometry because the corneal surface is transparent and the precorneal tear film is specular reflecting. There exist some techniques of which the feasibility has been proven. The most impressive are those that are based upon interferometry. ${ }^{1,2}$ However, due to the difficulty of aligning the patient's eye with the axis of the instrument and the extreme sensitivity to movement artifacts, these technologies have not been adopted in clinical practice so far. With a slitlamp one can obtain a profile from the cornea using Rayleigh scattering from the corneal tissue. ${ }^{3}$ By imaging multiple vertical slits across the whole width of the cornea from a known angle, this technology has been extended to a fully computerized system in the commercially available Orbscan. ${ }^{4}$ Another alternative to the classical Placido-based topographers is the rasterstereography as is used in the PAR corneal topographer.5,6 This system uses a grid pattern composed of horizontal and vertical lines spaced approximately $200 \mu \mathrm{m}$ apart. The topographic elevation is calculated from an image of the projected grid onto the corneal surface. Because the cornea consists of a transparent structure reflecting the light specular, its surface needs to be made diffusely radiating. This can be done by instilling sodium fluorescein in the precorneal tear film. It has been reported that the stability of the precorneal tear film is affected by fluorescein. ${ }^{7}$ Therefore, it is advised to perform in vivo exposures immediately after blinking. Tear filmdependent techniques do not work on dry eyes or other tear film deficiencies. Adding more viscous substances may change the surface shape ${ }^{8}$, but is not necessary for normal tear films. In case no coherent tear film is present, a diffusing membrane may be used. ${ }^{9}$ The fluorescent coating technique was already applied in 1979 by Kawara ${ }^{10}$ in a corneal topography device that uses moiré contour fringes. Moiré fringes can be observed when two gratings are superimposed (Fig. 1). This phenomenon can be used to obtain a display of the threedimensional shape of an object.

Kawara ${ }^{10}$ used sine wave gratings in his device (Fig. 2). A grating (PG) is projected on the fluorescein-coated tear film and modified by the corneal shape. The modified grating is then optically projected onto a reference grating (RG), positioned in an intermediate plane in the viewing system. As a result of the interference between the two gratings, moiré fringes are 


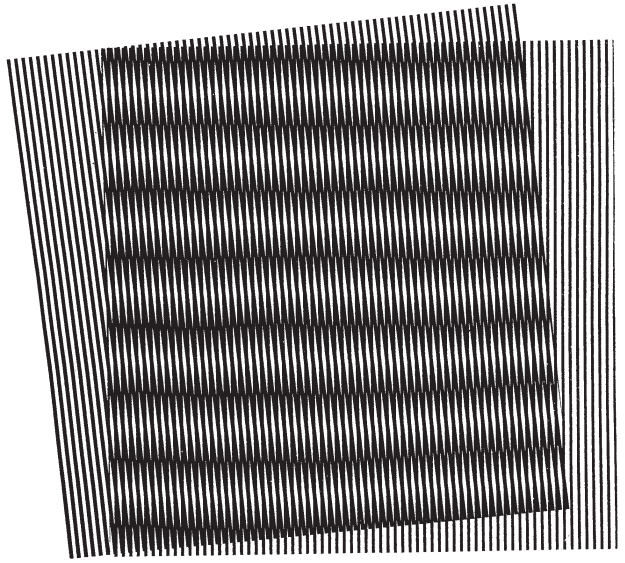

Figure 1. When two gratings are superimposed at an angle, a secondary pattern emerges that is called "moiré".

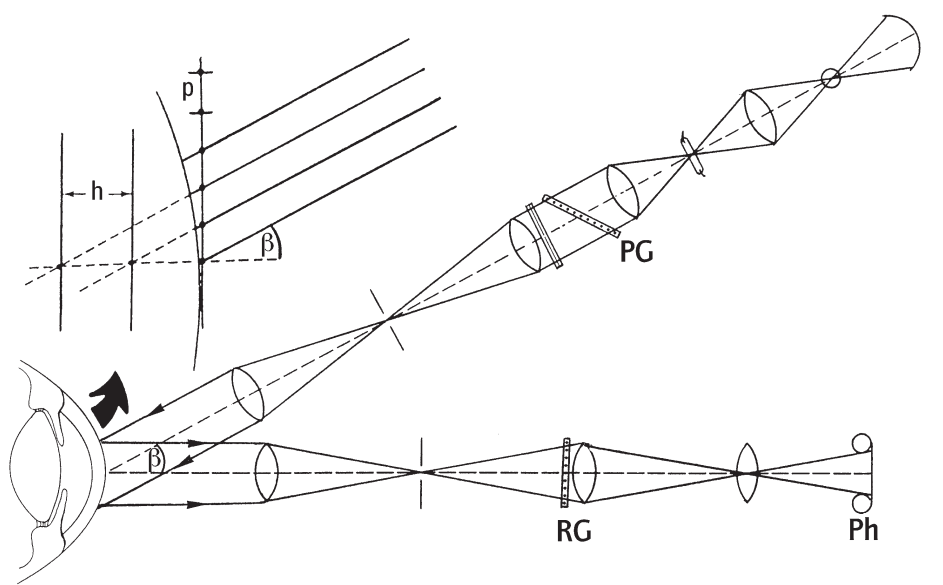

Figure 2. Schematic diagram of Kawara's moiré system. The moiré image is located at the reference grid (RG). Lines of equal height are obtained according $h=p / \sin \beta$ (see insert).

formed and photographed $(\mathrm{Ph})$. As the moiré image consists of lines of equal height, direct topographic information can be obtained without mathematical assumptions on the measured shape. In order to make magnification independent of distance, a telecentric projection method is applied. This provides an orthoscopic projection of the two grids on the corneal surface. The oblique orientation of the grating with respect to the optical axis of the projector corresponds with the Scheimpflug correction for oblique projection. Unfortunately, this correction method fails when narrow telecentric slits are used to obtain an orthoscopic projection. The location of the moiré contour fringes is precisely determined with microdensitometer traces from photographic recordings. Each measurement point has, according to Kawara, an accuracy of $+/-5 \mu \mathrm{m}$. Although this point accuracy is adequate for clinical prac- 
tice, the laborious technology requires automation for widespread use.

We used Kawara's design as a starting point for the development of a computer-assisted topographer that is able to determine the shape of the entire anterior surface of the eye without assumptions and with equal accuracy in the center and the periphery. The aim of the study was to test the width and depth of field, validity, and reproducibility of the instrument developed. At this stage, bispherical eye models were used that represent the broad range of corneal and scleral curvatures.

\subsubsection{Methods}

\section{Design considerations}

Projection-type moiré technologies have some inherent limitations:

1. The number of contour lines is dependent upon the spatial frequency and the angular displacement of the gratings used.

2. The spatial frequency of the moiré gratings is limited to the maximum frequency that allows the plus and minus first diffraction orders to pass the aperture of the projector. When a small slit is applied in the projector, the spatial frequency of the projected grid is consequently low. Kawara ${ }^{10}$ used about 12 cycles $/ \mathrm{mm}$, being about the physically maximum possible grid frequency with respect to the required depth of field. By tolerating a blur in the projected image equal to $1 / 4$ cycle of the projected grating, the modulation of the intensity is reduced to about $54 \%$. This arbitrary value was used as a boundary condition for the calculation of the required aperture dimensions, in order to obtain a depth of field of $6 \mathrm{~mm}$. The diffraction limit of the projectors can be calculated by determining the width of the slit necessary for passing the + and - first diffraction orders of the grating. The calculation, according to Abbe, assumes a point light source in the projection device. When the dimensions of the light source are also included in the calculation, a slightly smaller aperture can be used. The area limited by the boundary conditions mentioned is given in Fig. 3.

3. Moiré contour fringes are only located at depth intervals of $2 \pi$ or, when two opposed gratings with the same spatial frequency are applied, of $\pi$. This implies a restriction of the number of measuring points with respect to the uncertainty in each point.

4. To produce moiré difference contours without ambiguity, i.e., no spurious moiré fringes, the image to be sampled must have a spectrum that lies totally within the range of $1 / 2$ to 1 Nyquist frequency. ${ }^{11}$

5. The small slit that is required for projecting minute gratings with enough depth of field make projectors in a sensitive moiré system rather light consuming.

The number of measuring points and, consequently the accuracy of a moiré system can be extended by applying a scanning grid. ${ }^{12}$ Another method is to shift the object during the observation ${ }^{13}$ to reveal the local phase representing the instantaneous height of the contour fringes. However, to avoid movement artifacts, exposure times for corneal topography must be in the order of milliseconds, making it difficult to reveal the phase with a sequence of 


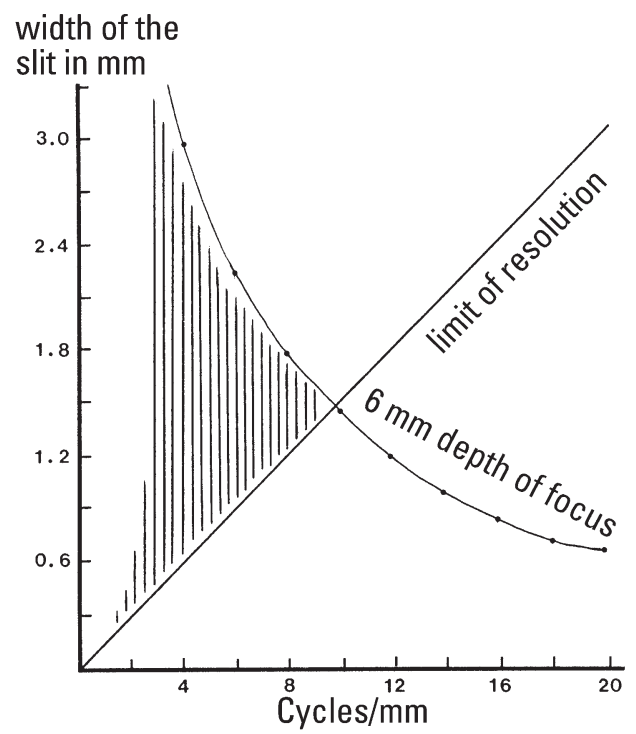

Figure 3. Slit width vs spatial frequency of the projected gratings. With $6-\mathrm{mm}$ depth of focus at a 1:1 imaging ratio, only the shaded area is available.

recordings. A mathematical technique for local phase reconstruction is the Fourier-transform method. Phase values can often be obtained, using sophisticated techniques, with an accuracy approaching $2 \pi / 1000 .{ }^{14} \mathrm{~A}$ disadvantage of these techniques is that they require moving parts or a series of phase related exposures. With a Fourier-transform analysis using only a single exposure, the image reconstruction is less accurate. However, Takeda et al. ${ }^{15}$ developed a method using only a single exposure with better than $2 \pi / 30$ point precision. Applied to height contouring also the sign of the phase shift can be revealed. This method works best on frequency- modulated signals. However, the amplitude modulation inherent to moiré techniques introduces noise.

Moiré amplitude modulation can be avoided by using two projections separated in time or by (color)labeling of the gratings for subtraction afterward. Another possibility is separation in space, which requires two sensors placed in opposite direction of the projection axis of one grating.

\section{The device}

With the above mentioned considerations in mind, we started to build a topographic device that could meet the criteria as given in Table 1. This was done at the Maastricht University in the Netherlands. The prototype instrument we present in this paper is therefore referred to as the Maastricht Shape Topographer (MST).

Fig. 4 shows an optical schematic of the MST. ${ }^{16}$ This topographer consist of two lateral telecentric sine wave grating projectors for the projection of orthoscopic images on the cornea in conjunction with a central orthoscopic viewing system. A rectangular diaphragm (RD), 


\section{Table 1. Requirements of the new topographic device.}

1. It must provide an accurate representation of the shape of the entire eye.

2. It must have enough depth of field to provide height data from adjacent tissues, such as limbal area and sclera.

3. Accuracy and reproducibility must be good enough to meet accepted clinical standards.

of which the long sides run parallel to the lines of the gratings, is placed in the confocal plane of the orthoscopic projectors. In this way, the gratings are projected such that a depth of field of $6 \mathrm{~mm}$ with respect to the viewing part is provided. A frame transfer CCD camera, with its optical axis bisecting the angle between the two lateral projectors, records the two sequentially flashed images as odd and even line fields, respectively. The time interval between the two images is about $8 \mathrm{~ms}$. The images are captured by a 512 x 512 pixel frame grabber (FG), and stored in a PC for analyzing. A pilot monitor (MON) enables rough focusing and centering on the eye. It also displays a composed image after exposure. The lateral projectors have an angular separation with respect to the detector of $18^{\circ}$. Each projector contains a $2.5 \mathrm{cycle} / \mathrm{mm}$ grating, which is projected on the precorneal tear film with a

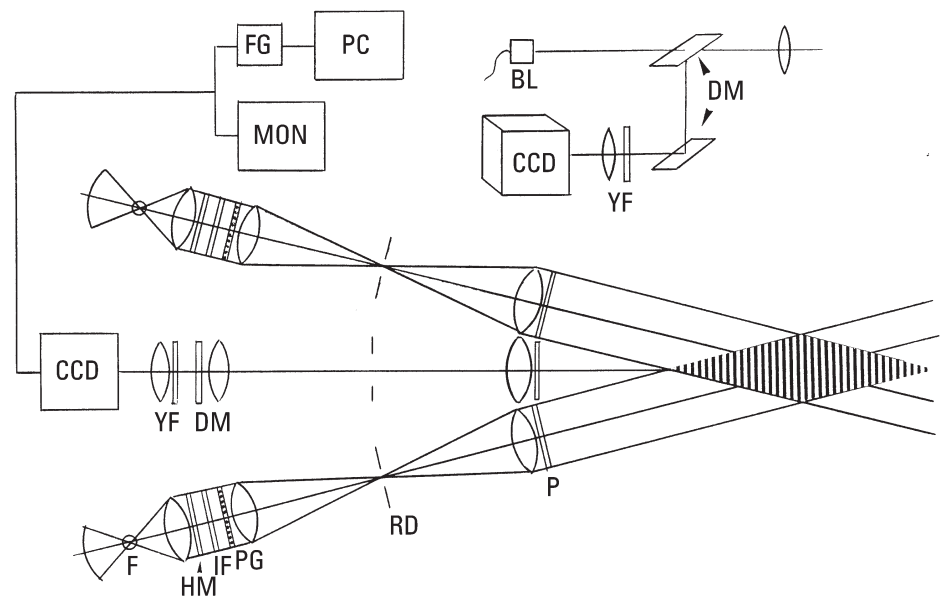

Figure 4. Optical schematic of the MST. The flash light in the lateral projectors is filtered by a heat reflecting mirror (HM) and a long pass interference filter (IF) that blocks radiation with a wavelength longer than 480nm. The sine-wave grating (PG.) is projected onto the anterior surface of the subject's eye. A vertical slit (RD) serves as the telecentric diaphragm. A crossed polarizer with respect to the polarizers $(P)$ on the projectors is placed in the viewing path in order to suppress specular reflection. Further suppression of unwanted light is obtained by a yellow filter (YF) that is nearly opaque for the excitation light. With dielectric mirrors (DM) the fluorescent light is guided to the camera while the blue led (BL) is visible for aligning the subject's eye (see insert). The CCD camera is connected with a monitor for focusing and a frame grabber (FG) for connection to a PC. The subjects' eye must be placed in the area where the two projected gratings coincide (indicated with vertical parallel lines). 
1:1 imaging ratio. Thus, a moiré fringe represents a depth interval of about $0.65 \mathrm{~mm}$, with respect to a reference plane perpendicular to the optical axis of the sensor. The exposure time is determined by a $0.1-\mathrm{ms}$ flash of light generated by a xenon tube in the projector. Between the flash tube (F) and the grating (PG) a heat reflecting mirror (HM) and an interference filter (IF) are inserted to restrict the radiation load on the eye to that of the blue-green excitation light. The fluorescent images from the modified grating are guided by two dichroic mirrors (DM), which reflect wavelengths longer than $500 \mathrm{~nm}$ to the detector. In order to increase the suppression of the excitation light, a yellow barrier filter (YF) is placed in the viewing optics. Specular reflections are further suppressed by polarizers $(\mathrm{P})$ placed in the projectors in conjunction with a crossed polarizer in the viewing optics of the sensor. Without the polarizers, a local saturation of the CCD sensor causing loss of data may be introduced by specular reflection. This would prevent unambiguous phase unwrapping necessary for the height reconstruction. The standard UV polarizers were the only components in the device that affected the etendue (matching of the apertures of an optical system) of the projectors and recording system. However, to prevent local overexposure, this shortcoming was accepted. A blue led (BL) is placed so as to permit the subjects' eye to be aligned with the optical axis of the sensor. The independent odd and even frames are analyzed separately using Fourier techniques ${ }^{16}$, before being combined in a later stage of the reconstruction process.

A diffuse infrared illumination is added to locate the target area. Exact focussing is performed by triangulation with two diode lasers that are mounted onto the projectors. The 0.5 $\mathrm{mW}$ output of these diode lasers is filtered by a $1 \%$ transmission neutral density filter and focused on the conjunctiva near the limbal area of the subject's eye by an $f=160 \mathrm{~mm}$ lens. The software in the MST for the image reconstruction is based upon Takeda et al. and extended for use in the MST. ${ }^{17}$ The surface shape can be displayed either as a three dimensional $(\mathrm{x}, \mathrm{y}, \mathrm{z})$ contour map or as cross-section. As the three-dimensional mappings are calculated for Cartesian coordinates, the display can be manipulated to enhance particular features such as image subtraction and reset of the center of the reconstruction to relocate the corneal apex.

Because of the oblique projection, the peripheral left part of the left projection and the peripheral right part of the right projection may be out of focus. As both half frames of the TV system are used for the reconstruction, the missing information from one half frame can be compensated by the other frame.

\section{Calibration and validation of the MST}

On a computer-assisted submicron precision lathe (Euro Precision Technology), three bispherical polymethyl methacrylate molds were produced. The total diameter of the molds was $20 \mathrm{~mm}$; the central part representing the cornea had a diameter of $12 \mathrm{~mm}$. One mold had a "corneal" radius of $7 \mathrm{~mm}$, with a "scleral" radius of $13 \mathrm{~mm}$. The other two molds had radii of $8 \mathrm{~mm} / 12 \mathrm{~mm}$, and $9 \mathrm{~mm} / 15 \mathrm{~mm}$, respectively. From the molds impressions were made with vinyl polysiloxane $\left(3 \mathrm{M}\right.$ Express $\left.^{\mathrm{TM}} 7301 \mathrm{H}\right)$ that has the property to show autofluorescence when exposed to blue light. Using this material, we obtained eye models that are entirely fluorescent, without the need for a coating or application of fluorescein. The 
model had a flat ground surface perpendicular to the central axis and is fixated in a microbench adapter plate (Spindler \& Hoyer). To be able to control rotation exactly, the adapter plate was placed in a divided circle rotating mount.

As, according to the manufacturer, some dimensional change may occur during the first 24 $\mathrm{h}$ after making the impression, the shape of the vinyl polysiloxane eye models was measured one day after molding. To be able to compare MST height values to a solid standard, measurements on the eye model were first carried out with a Leitz microscope. For this, the eye model in its mount was fixed on the microscope table. The $\mathrm{z}$ - and $\mathrm{x}$-axes of the microscope were connected to micrometers (Haidehahn Metrotaster $30 \mathrm{M}$, sensor resolution $\pm 0.5 \mu \mathrm{m}$ ). z Axis measurements were performed with a long free-working distance objective (Leitz UTK $32 \mathrm{x} / \mathrm{NA}=0.40$ ). The microscope was coupled to a TV-camera showing a $1300 \mathrm{x}$ magnified image of the surface of the eye model. In this way, focusing (z-axis) was done at an 8 - $\mu \mathrm{m}$ diameter area of the model with a focusing accuracy of $\pm 1 \mu \mathrm{m}$. Guided by the monitor image and the Metrotaster readings, the highest point of the eye models was found in an iterative $z-x$ and $z-y$ search. On both sides of the highest point, $z$ measurements were taken, thus scanning the whole meridian, at intervals of $500 \mu \mathrm{m}$. After the series of measurements along a meridian was completed, the top was again brought in focus (zero value on the Metrotaster). When in that stage more than $1 \mu \mathrm{m}$ refocusing had to be done, the measurement series was rejected. Then, the mold was rotated $90^{\circ}$ and the measuring procedure repeated.

Because of the contact lens production program of the lathe, the transition area between diameter 10 and $14 \mathrm{~mm}$ could not be defined, and therefore measurements in that area are omitted. Hence, we used a $10-\mathrm{mm}$ central area (20 measuring points per meridian) and a peripheral area extending from 14 to $19 \mathrm{~mm}$ (22 measuring points per meridian). If we use "central" or "peripheral" in the following text, we refer to these figures.

Directly after the measurement with the microscope, five exposures from each model were made with the MST. Before measuring, the MST was calibrated on a precision length caliber. In order to be able to compare the MST and microscope height measurements, the same meridians $\left(0-180^{\circ}\right.$ and $\left.90-270^{\circ}\right)$ were sampled at intervals of $500 \mu \mathrm{m}$. The height data of the MST are recorded after finding the highest point of the eye model in a process similar to that used with the microscope.

The reproducibility of the MST was tested by analyzing the variance among the five measurements for the three eye models in the center and in the periphery. To compare height measurements, the average value of the five measurements with the MST at each point was compared with the value at the same point obtained by the microscope. To compare radii, we computed axial radius of curvature (AROC) from each measured $\mathrm{z}$ value. AROC values were averaged to obtain one value for the central or peripheral part of the eye models.

\subsubsection{Results}

\section{Accuracy and precision in height}

The accuracy in height is given in Table 2. The right columns show the average differences 


\begin{tabular}{|c|c|c|c|c|c|}
\hline \multirow{2}{*}{$\begin{array}{l}\text { Area } \\
(\mathrm{mm}) \\
\end{array}$} & \multirow{2}{*}{$\begin{array}{l}\text { Radius } \\
\text { mold } \\
(\mathrm{mm})\end{array}$} & \multicolumn{2}{|c|}{$\begin{array}{l}\text { Reproducibility: } \\
\text { Standard Error MST }\end{array}$} & \multicolumn{2}{|c|}{$\begin{array}{l}\text { Accuracy: } \\
\text { Difference in height Microscope-MST }\end{array}$} \\
\hline & & $\begin{array}{l}\text { Average } \\
(\mu \mathrm{m})\end{array}$ & $\begin{array}{l}\text { SD } \\
(\mu \mathrm{m})\end{array}$ & $\begin{array}{l}\text { Average } \\
(\mu \mathrm{m})\end{array}$ & $\begin{array}{l}\text { SD } \\
(\mu \mathrm{m})\end{array}$ \\
\hline 10 & 7 & 0.018 & 0.022 & -0.369 & 2.901 \\
\hline 10 & 8 & 0.003 & 0.002 & -0.551 & 4.890 \\
\hline 10 & 9 & 0.063 & 0.072 & 0.548 & 1.563 \\
\hline $14-19$ & 12 & 4.633 & 8.225 & 0.017 & 62.458 \\
\hline $14-19$ & 13 & 3.025 & 2.060 & -44.155 & 19.897 \\
\hline $14-19$ & 15 & 0.189 & 0.152 & 1.021 & 13.018 \\
\hline
\end{tabular}

and standard deviation for the central and peripheral parts of the three eye models. From these data it can be seen that the accuracy in the central $10-\mathrm{mm}$ zone is high and the differences between the models are small. The data are consistent and do not statistically differ (Student $\mathrm{t}$-test $\mathrm{p}>0.05$ ). The 14- to 19 -mm peripheral zone shows more inconsistency. Although the differences for radii 12 and 15 are not significantly different from the central part data, they do differ significantly (Student $\mathrm{t}$-test $\mathrm{p}<0.05$ ) from the data for the $13-\mathrm{mm}$ eye model. Because of the local underexposure caused by the obstructing polarizers, there is remarkable variation in standard deviations for the periphery. The interpretation of differences between average differences is difficult. Also, although the reproducibility of the microscope measurements was within $\pm 1 \mu \mathrm{m}$, the use of the microscope is new and cannot be taken as the accepted "gold standard".

For these reasons, we extended the analysis, using the Bland and Altmann ${ }^{18}$ method for comparing two measuring methods. Fig. 5 shows a scatter plot of the average between the microscope and MST height value against the difference between the two measurements. Note that the right part of the plot corresponds to the peripheral (scleral) values. The solid line $(-0.0083 \mathrm{~mm})$ shows the overall average, with a $95 \%$ interval of agreement [shaded area $= \pm 1.96 * 0.0212(\mathrm{SD})]$. Because of a transition zone between the central and peripheral curves, measurements between 5 to $6.5 \mathrm{~mm}$ from the center are eliminated. The data show good agreement within the central $10 \mathrm{~mm}$ area with more scatter in the periphery. Fig. 6 shows these data in more detail for the central $10-\mathrm{mm}$ (corneal) area only. Here the average difference was $-0.0001 \mathrm{~mm}$, with SD of 0.00344 .

\section{Reproducibility}

Five sequential operator-independent measurements were made, thus without touching the eye model or changing the focusing of the MST. The Reproducibility columns of Table 2 show the reproducibility as average standard error (with its SD) for the whole central and peripheral part of each eye model. Taken over all eye models, the average standard error in 


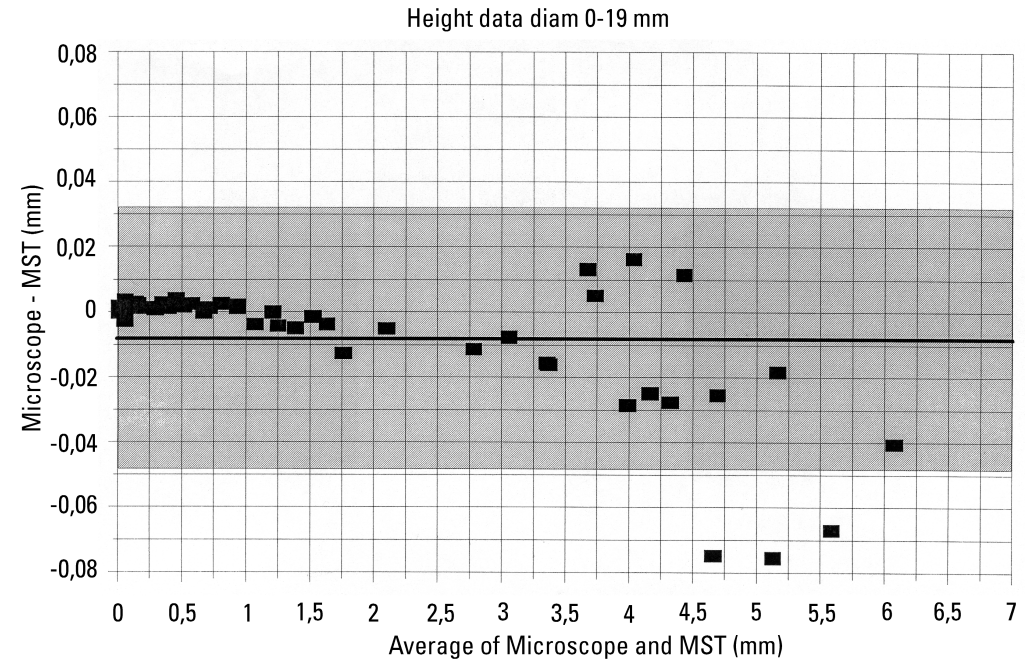

Figure 5. Scatterplot of the average between the microscope and MST height values against the difference between the two measurements. The solid line $(-0.0083 \mathrm{~mm})$ shows the overall average with $95 \%$ interval of agreement (shaded area).

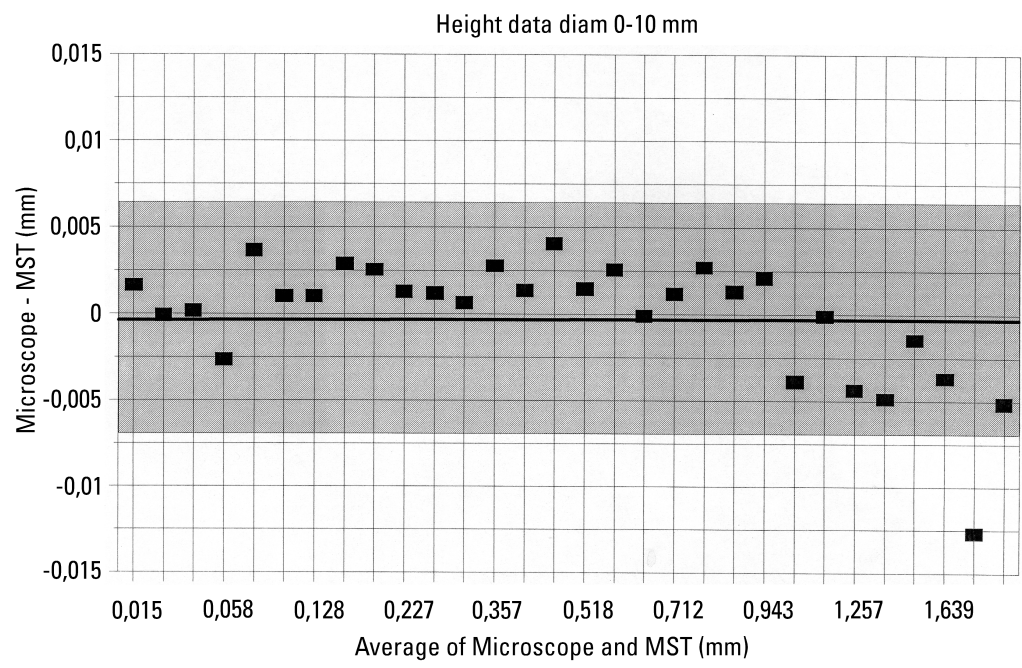

Figure 6. Scatter plot of the average between the microscope and MST height value against the difference between the two measurements for the central $10 \mathrm{~mm}$. The solid line $(-0.001 \mathrm{~mm})$ shows the overall average with $95 \%$ interval of agreement (shaded area). 
the $10-\mathrm{mm}$ central area was $0.0282 \mu \mathrm{m}$ (range 0.003 to 0.063 ) and for the peripheral 14-to $19 \mathrm{~mm}$ area, $2.6156 \mu \mathrm{m}$ (range 0.180 to 4.633). The standard errors show no statistical differences among each other for the individual eye models at the central 10-mm part (Student $\mathrm{t}$-test, $\mathrm{p}>0.05$ ). For the periphery, there is no statistical difference (Student t-test, $\mathrm{p}>0.05$ ) between the standard error for the 12- and 13-mm eye models. However, both show a statistical difference with the central standard error values and with the standard error found for the 15 -mm eye model (Student $\mathrm{t}$-test $\mathrm{p}<0.05$ ).

\section{Conversion of height values in radius of curvature}

AROC was calculated, using the microscope height value and the mean MST value in each point $(500 \mu \mathrm{m}$ intervals). Conversion from height to radius from small sagittal values may introduce significant error because of the reciprocal relationship between curvature and sag. Therefore, in the central area radii were computed from a point $2 \mathrm{~mm}$ from the center. The computed AROC values were then averaged over the area. The variation is given as standard deviation. The same method was used to calculate peripheral radii over an area of 7.0 to 9.5 $\mathrm{mm}$ from the center. The results are shown in Table 3. Average reconstruction accuracy of the radius of curvature of the MST compared to the microscope was $\pm 0.0155 \mathrm{~mm}$ for the central $10 \mathrm{~mm}$ and $\pm 0.0313 \mathrm{~mm}$ in the periphery ( 14 to $19 \mathrm{~mm}$ ). The central (corneal) radius of curvature reconstruction accuracy may be expressed in diopters. Assuming an index of refraction of 1.3375 , the accuracy is $\pm 0.08 \mathrm{D}$.

Table 3. Averages, SD and differences in radius of curvature of 3 bi-spherical eye models as calculated from the height data of the microscope and the MST. ${ }^{a}$

\begin{tabular}{|c|c|c|c|c|c|}
\hline \multirow{2}{*}{$\begin{array}{l}\text { Eye models } \\
\text { Mold }(r)\end{array}$} & \multirow[t]{2}{*}{ Microscope } & \multirow[t]{2}{*}{ MST } & \multirow{2}{*}{$\begin{array}{l}\text { Difference: } \\
\text { Microscope-MST }\end{array}$} & \multicolumn{2}{|c|}{ SD } \\
\hline & & & & Microscope & MST \\
\hline 7 & 6.990 & 7.025 & -0.035 & 0.0137 & 0.0148 \\
\hline 8 & 7.989 & 7.993 & -0.004 & 0.0119 & 0.0262 \\
\hline 9 & 8.926 & 8.947 & -0.022 & 0.0333 & 0.0135 \\
\hline 12 & 12.024 & 11.985 & 0.040 & 0.0464 & 0.1200 \\
\hline 13 & 12.985 & 12.926 & 0.059 & 0.0058 & 0.0527 \\
\hline 15 & 14.958 & 14.949 & 0.009 & 0.0111 & 0.0756 \\
\hline \multicolumn{6}{|c|}{$\begin{array}{l}{ }^{a} \text { All values are in mm. Column } 1 \text { gives the radii of the bi-spherical molds to produce the eye models. The } \\
\text { average radii as calculated from height measurements with the microscope and the MST are given in columns } \\
2 \text { and 3, respectively; column } 4 \text { shows the differences between these values, whereas columns } 5 \text { and } 6 \text { give } \\
\text { the SD of the calculated set of radii for the microscope and the MST. }\end{array}$} \\
\hline
\end{tabular}

\section{Depth of field}

The entire corneal surface and adjacent tissue can be measured, limited by either the depth of field or the angle of view of the device. For a sine wave projection with a $400 \mu \mathrm{m}$ period as used in the MST, the depth of field exceeds $6 \mathrm{~mm}$, allowing a significant part of the scle- 
ral-conjunctiva tissue to be in focus. Fig. 7 shows a cross-section height profile of an eye model and Fig. 8 shows a cross-section height profile of the anterior eye of one of the authors. The limbal transition area of the eye model separates two bispheric curves. The in vivo limbal transition separates the smooth corneal surface from the more irregular scleralconjunctiva surface. Also the nasal/temporal asymmetry can be seen.

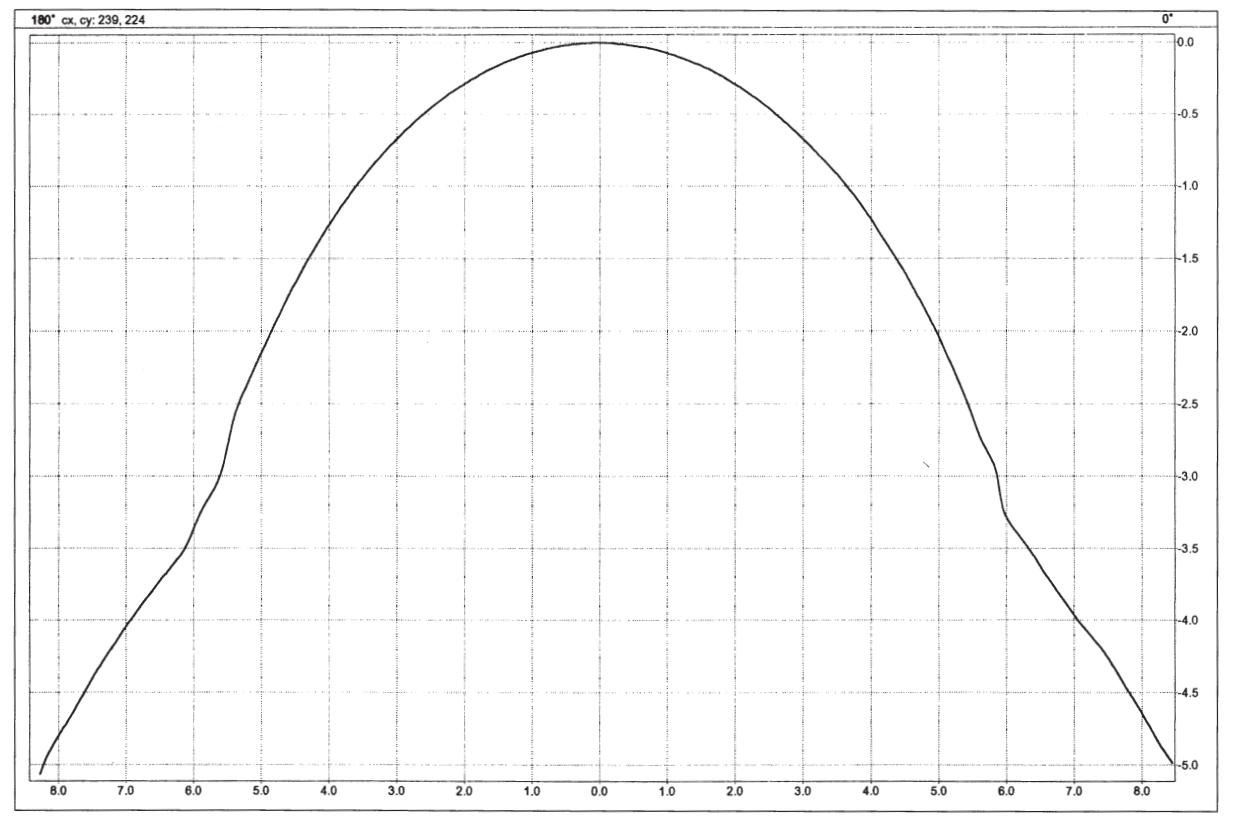

Figure 7. Cross section of the surface of a bispherical eye model. Note: $x, y, z$ ratio has been changed for illustrative purpose.

\subsubsection{Discussion}

A height measuring device, like the MST, has the advantage that the primary data obtained are nonparametric (model free). In the MST, the number of measuring points equals the number of pixels of the CCD sensor. A lateral resolution equal to the resolution of the sensor can be obtained. The accuracy of the MST depends on the characteristics of the sensor, the spatial frequency of the gratings, and the calculations to reconstruct the topography. The pixels are correlated using a Fourier transform-based reconstruction algorithm, similar to that of Takeda et al. ${ }^{15}$ In this way, it is possible to guarantee pixel resolution. This is illustrated in Fig. 9, showing an enlargement of the central $1-\mathrm{mm}$ area of the 0 to $180^{\circ}$ meridian of a living eye. If accuracy is high throughout the field, the use of subpixel resolution techniques could be of interest to increase local discrimination of shape. 


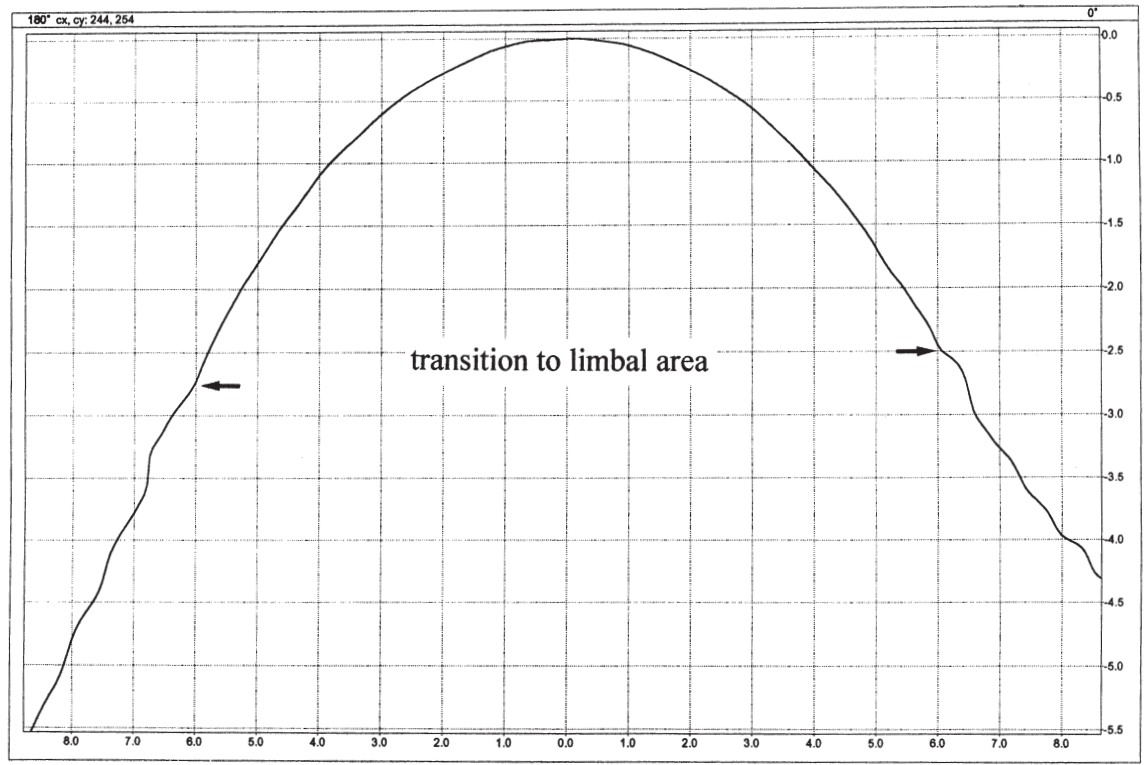

Figure 8. Cross section of the anterior surface of the right eye of one of the authors. Note: $x, y, z$ ratio has been changed for illustrative purpose.

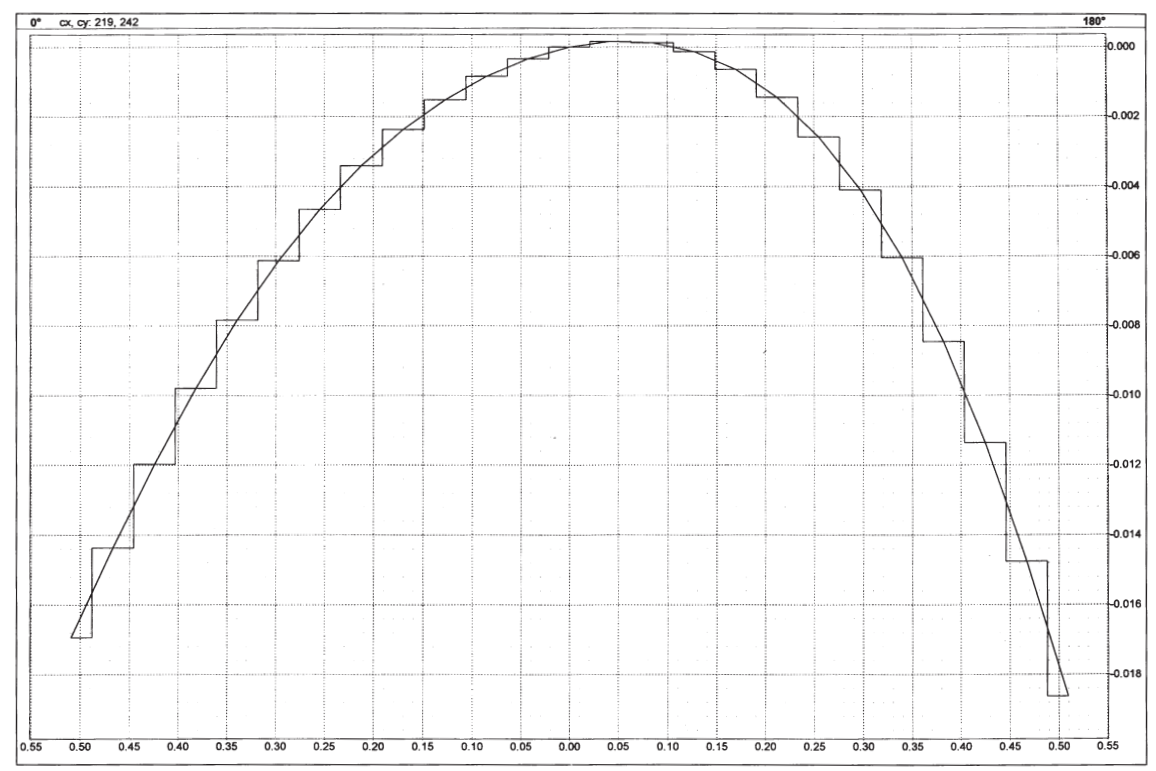

Figure 9. Enlargement of a horizontal cross-section of the surface of a living eye. Height values at pixel level are shown for the central part of $1 \mathrm{~mm}$. Note $x, y, z$ ratio has been changed for illustrative purposes. 
The prototype MST was made of standard components with small diameter polarizers that affected the etendue of the viewing system. In this way, some vignetting was introduced that caused poor signal to noise ratio values for the peripheral measurements (Table 2). To solve this technical problem, custom-made polarizers could be used. Another possibility could be the use of a combination of excitation and barrier filters that have a higher capacity to block the disturbing specular reflection.

Our aim in this study was to validate the MST on the ability to measure height with a large field. For this reason, we restricted measurements to bispherical models representing the entire surface of the eye. On purpose we did not include aspherical and irregular eye models, in vivo measurements, and inter-observer variability at the present stage. We realize, however, that this should be subject to investigation in further validation and reproducibility testing of the device. This should also include other means of presentation of data like instantaneous radius of curvature, corneal peripheral flattening, astigmatism, and irregularity indices.

We have analyzed the data with the standard Student t-test. This follows the method as described by Hanusch et al. ${ }^{19}$ From statistical theory, however, it can be argued that the variance at the center and the periphery of the eye models may not follow a normal distribution. An analysis of variance on all data did not show any other conclusions than the Student t-test method. A more sophisticated analysis using the multivariate nature of data according to Viana et al. ${ }^{20}$ and incorporating more measuring points could possibly resolve this statistical problem. We did not apply this method in the present research because by resolving the technical problem of vignetting, as outlined before, the difference in central and peripheral height accuracy would probably be eliminated.

\subsubsection{Summary}

With the MST, unambiguous shape measurements of the models representing the anterior eye surface were possible.

The MST has a depth of field of $6 \mathrm{~mm}$, and is able to measure height over the entire 20$\mathrm{mm}$ field. This feature enables the device to measure not only the entire corneal surface but also the limbal and scleral-conjunctiva surface.

This research shows that for the central area of $10 \mathrm{~mm}$, the prototype of the MST has sufficient accuracy and reproducibility. For the periphery, the device in its present state works satisfactorily in terms of radius but could be further improved on peripheral height accuracy. Because the MST eye topographer offers the possibility of extended measurements in height over a large field, its use could be advantageous in cases where shape is of paramount importance, e.g., in (scleral) contact lens fitting and refractive surgery.

\section{Acknowledgement}

We thank Prof. dr. D.W. Slaaf of the Departement of Biophysics of the University Maastricht for the hospitality in his Laboratory for Microcirculation, enabling us to perform the microscopic measurements. Also, we thank Ben Lubberman of Sumipro and George Lo- 
A-Foe of Euro Precision Technology for their help in fabricating the molds. This study was partly supported by Technisch Ontwikkelings Krediet 91073 from the Ministry of Economic Affairs of the Netherlands. A clinical evaluation of the device has been performed at St. Thomas's Hospital in London, during 1 year, on patients referred for topography. ${ }^{21}$

\section{References}

1. Politch J. Optical and long wave holography potential application. Doc Ophthalmol 1977;43:165-75.

2. Kasprzak H, Förster WN, von Bally G. Holographic measurement of changes of the central corneal curvature due to intraocular pressure differences. Opt Eng 1994;33:198-203.

3. Mandell RB. Profile methods of measuring corneal curvature. J Amer Opt Assoc 1961;32:627-31.

4. Mayberry M, Zimmerman MB, Mathers WD, Sutphin JE. Detection of keratoconus using Orbscan scanning slit topography. Invest Ophthalmol Vis Sci 1997;38:s920.

5. Cambier JL, Strods SJ. Method and apparatus for obtaining the topography of an object. Patent PCT/US91/04960, 15 July, 1991.

6. Belin MW. Intraoperative raster photogrammetry, the PAR corneal topography system. J Cataract Refr Surg Supplement 1993;19(S):188-92.

7. Patel S, Murray D, Mc Kenzie A, Shearer DS, Mc Grath BD. Effects of fluorescein on tear film breakup time and on tear thinning time. Am J Optom Physiol Opt 1985;62:188-90.

8. Pavlopoulos GP, Horn J, and Feldman ST. The effect of artificial tears on computer-assisted corneal topography in normal eyes and after pentrating keratoplasty. Am J Opthalmol 1995;119:712-22

9. Lange SR and Thall EH. Method and apparatus for measuring corneal topography. US patent No 4,984,893 Jan. 15, 1991.

10. Kawara T. Corneal topography using moiré contour fringes. Applied Optics 1979;18:3675-8.

11. Bell BW and Koliopoulos CL. Moiré topography, sampling theory, and charged-coupled devices. Optics Letters 1984;9(5):171-3.

12. Hovanesan JD, Hung YY, and Waidelich W. Moiré contouring of large objects by a scanning technique. Proc. Laser '77 Opto-electronics, Munich, Germany, 20-24 June 1977. Laser 77,1977:589-97.

13. Jüptner W, Kreis Th, and Kreitlow $\mathrm{H}$. Automatic evaluation of holographic interferograms by reference beam phase shifting. Proc Soc Photo-Opt Instrum Eng 1983;398:22-9.

14. Reid GT. Automatic Fringe Pattern Analysis: A Review. Optics and Lasers in Engineering 1986;7:3768.

15. Takeda M, Ina H, and Kobayashi S, Fourier-transform method of fringe-pattern analysis for computer-based topography and interferometry. J of Opt Soc of Am 1982;72;1:156-60.

16. Jongsma FHM. System for determining the topography of a curved surface. United States Patent Number 5,406,342, April 11, 1995.

17. Stultiens BATh, Jongsma FHM. Frequency modulation as an alternative for local phase in $3 \mathrm{D}$ corneal topography. Progress in Biomedical Optics Proceedings series, SPIE Proc of Ophthalmic Technologies IV JM Parel, Q Ren, A Katzir (ed), 22-23 January 1994 Los Angeles, Ca 1994;2126:174-84.

18. Bland JM, Altman DG. Statistical methods for assessing agreement between two methods of clinical measurements. Lancet 1986;1:307-10.

19. Hannush SB, et al. Accuracy and precision of keratometry, photokeratoscopy, and corneal modeling on calibrated steel balls. Arch Ophthalmol 1989;107;8:1235-9. 
20. Viana MGA, Olkin I, Mc Mahon TT. Multivariate assessment of computer-analyzed corneal topographers. J Opt Soc Am 1993;10;8:1826-34.

21. Corbett MC, O’Brart DP, Stultiens BA, Jongsma FHM, Marshall J. Corneal topography using a new moiré image based sytem. Eur J Implant Ref Surg 1995;7:353-70. 


\subsubsection{Height Measurements of the Corneal-Scleral Profile using the Maastricht Shape Topographer}

J. de Brabander, R.M.M.A. Nuijts, F.H.M. Jongsma and F. Hendrikse.

From:

Eye research Institute Maastricht, Department of Ophthalmology, Academic Hospital University Maastricht, the Netherlands.

Submitted. 
Abstract

Purpose. Knowledge of the Corneal-Scleral Profile (CSP) is of importance in scleral and soft contact lens fitting. Eye impressions and photographic profiles are traditionally used to show CSP, qualitatively. Contrary to normal reflection video-keratography the Maastricht Shape Topographer (MST), based on Fourier Profilometry, is able to present a height map of cornea and sclera. The present study aims to measure and compare $\mathrm{x}, \mathrm{y}, \mathrm{z}$ values and to compute the slope of the limbal area in normal and keratoconus eyes using the MST eye topographer.

Methods. In each of 29 normal and 11 keratoconus eyes, five MST measurements of the cornea-scleral profile in the horizontal meridian were taken. The $\mathrm{x}$ and $\mathrm{z}$ values of the limbal area were averaged and the temporal and nasal slopes were computed.

Results. Limbal topography in the horizontal meridian of the eyes of all subjects could be obtained. The reproducibility of finding the $\mathrm{x}$ and $\mathrm{z}$ values of the defined limbal borders was within $30 \mu \mathrm{m}$. In keratoconus eyes the mean $\mathrm{z}$ value between the highest point of the cornea and the limbal borders was significantly higher $(0.31 \mathrm{~mm}, \mathrm{SD} \pm 0.094)$ than in normal eyes. In normal eyes the mean nasal slope of the limbal area was significantly lower than the temporal. This asymmetry was not found in keratoconus eyes.

Conclusion. Height measurements of the corneal-scleral profile with the MST device showed to be possible with good repeatability in the horizontal meridian. Contrary to normal eyes, the limbal area in keratoconus eyes showed no difference between nasal and temporal slope.

\section{Key words:}

Corneal-scleral profile, limbus, eye topography, keratoconus. 


\subsubsection{Introduction}

The Corneal-Scleral Profile (CSP) is of importance in relation to the fitting of scleral contact lenses. Fitting of scleral contact lenses traditionally involved the tailor modification of pre-formed scleral shells. ${ }^{1}$ The theory of fitting has been based on classical eye models with a spherical corneal curvature and an abrupt change in curvature at the limbus. This has been the basis of the so-called "haptic" scleral contact lens design, in which the base curve of the corneal portion of the lens after a transition zone transforms into a much flatter scleral portion.

Due to their large diameter, the fitting characteristics of soft contact lenses (SCLs) are more dependent on sagittal depth and peripheral shape of the eye than on central radius of the cornea. For this reason the CSP is of importance in fitting these types of CLs. Surprisingly, the fitting of SCLs lenses has drawn only little attention in the literature on CSP. This might be caused by the lack of accurate devices for measuring the shape of the corneal-scleral junction and the sclera itself.

Traditionally, obtaining eye impressions has been the method to gain inside into the shape of the eye to perform fitting of scleral contact lenses. Pastes, that originate from dental impression techniques (Kromopan or Panasil) are used to obtain negative impressions of the entire eye shape. ${ }^{2}$ From the impression a positive cast can be made and measured or photographed to reveal a profile view. Problems with this technique are corneal distortion due to pressure on application, fixation problems, eye movements, problems with removal of the substance, lack of reference axis and shrinkage of the cast. All these factors make the technique unsuitable for providing very exact information on CSP. Despite the limitations of the method it has been recognized that the assumed sharp limbal transition does not exist and that there are remarkable differences in nasal and temporal scleral shape and orientation of the center of curvature. ${ }^{3}$

Mandell ${ }^{4}$ was one of the first to analyze the CSP by photographic techniques. Meier and Gaggioni ${ }^{5}$ composed a subjective classification system for CSP from analysis of a large pool of profile-photographs. They concluded that the variation in CSP is large. Their classification is build on the observation of the cornea-scleral transition (fluent or outstanding) and the shape towards the sclera (convex, tangential or concave).

In Placido disk based computer aided video keratography (CAVK) the measured area does not include the outer parts of the cornea, limbus or sclera. The Maastricht Shape Topographer (MST) 6 , based on Fourier Profilometry, is able to present a height map of the total area exposed to the instrument (Fig. 1). With the MST it is possible to create cross sections of cornea and sclera including limbal topography (Fig. 2). The purpose of the present study is to obtain height measurements of the cornea and sclera in normal and keratoconus eyes and to analyze these data to find characteristics of CSP at the limbal area.

\subsubsection{Material and methods}

In the present study MST height topography was obtained from 29 normal and 11 keratoconus eyes. The normal group included eyes of adult patients (age $20-35$ years) with low 


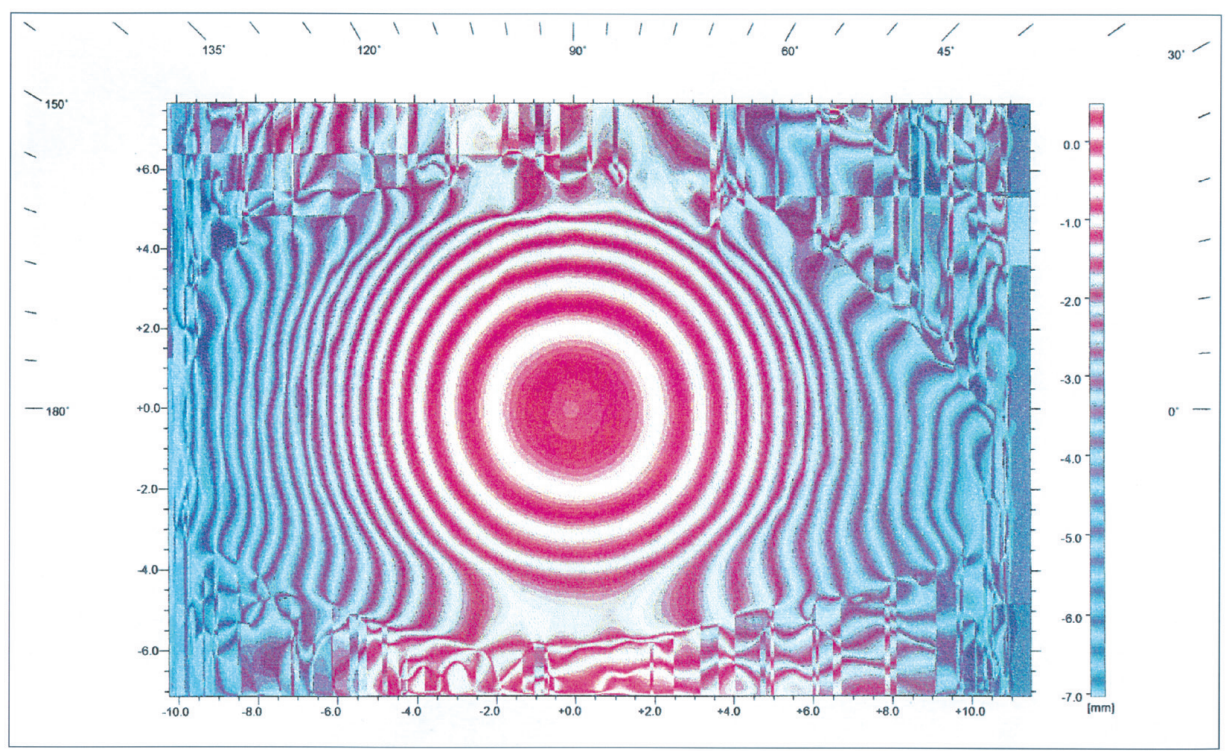

Figure 1. Height contours obtained with the Maastricht Shape Topographer (MST). The contours represent lines of equal height that can be translated into $x, y, z$ coordinates to describe the complete eye surface as exposed to the device.

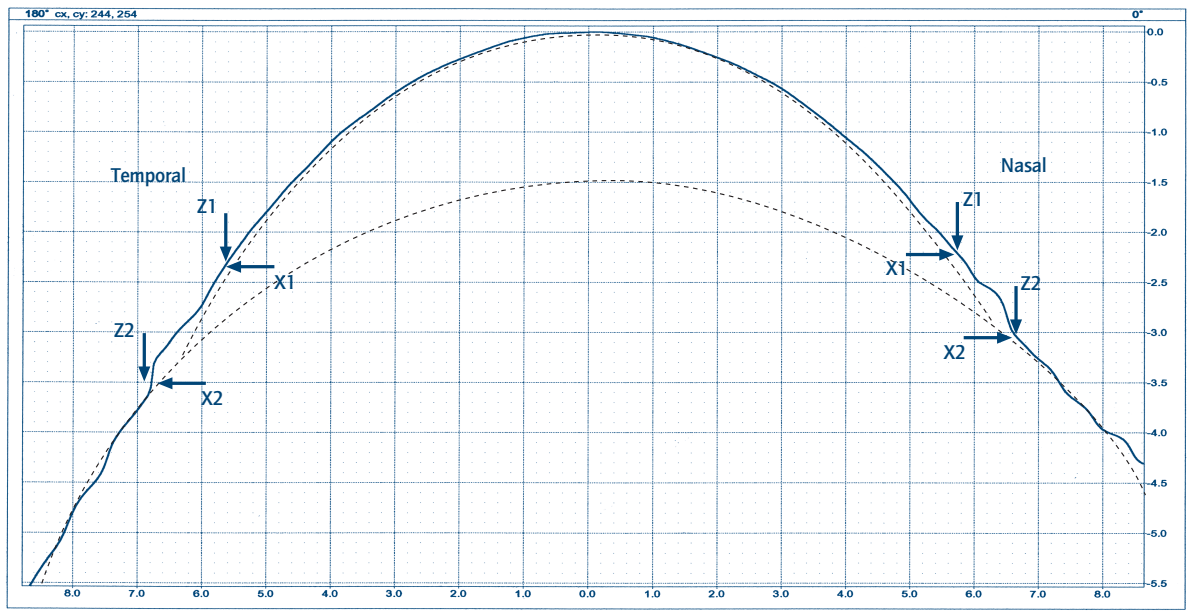

Figure 2. Cross section of a horizontal profile of a normal eye (OD). The zero value to measure the $z$ values is the highest point in the topography. The alignment matrix as given by the measuring device represents the central axis from which $x$ values are measured. The dashed lines represent the method of fitting that was used to find the $Z ; X$ coordinates of the limbal area. Note: for reasons of presentation the scaling for $z$ and $x$ values is not the same. Also, the dashed lines representing the corneal fit are slightly displaced downwards. 
ametropia $(<4.00 \mathrm{D})$ that had never worn contact lenses. The keratoconus group included eyes of patients in the same age group with a clear diagnosis of advanced keratoconus and no contact lens wear at least one month prior to the measurement. Originally, our method included a measurement with distended eye aperture in order to also image the vertical meridian beyond the limbal area. However, this method showed a much higher variability than normally found with the MST device. ${ }^{6}$ Probably the influence of manipulating the eye lids distorted the tear layer too much. We therefore made five repeated measurements of the height contours without manipulating the eye lids and only analyzed the horizontal meridians. An example of a horizontal cross section profile is shown in Fig. 2. On these cross sections both the nasal and temporal limbal areas were found using the definitions and method as described below. All $\mathrm{x}$ and $\mathrm{z}$ values are measured from the zero value as given by the highest point of the topography and the central axis of the matrix as given by the MST device. From the corneal side, the beginning of the limbal area was defined to start at the $\mathrm{z}$ value that deviates more than $0.10 \mathrm{~mm}$ from the extension of a best fitted ellipse on an area between 0 and $3.5 \mathrm{~mm}$ of each semi meridian (X1;Z1 in Fig. 2). To properly perform the elliptical fit, the corneal height data of a semi meridian under analysis were mirrored around the central axis to obtain a rotational symmetric body. This procedure avoids that in case of asymmetry the data per semi meridian are obscured by averaging over the entire meridian. From the scleral / conjunctival side, the beginning of the limbal area was defined as the point that differs more than $0.10 \mathrm{~mm}$ in $\mathrm{z}$ value from the extension of a best fitted sphere on the scleral profile beyond $7 \mathrm{~mm}$ from the center. The orientation of the spherical fit is the central axis and the aim of the procedure is to find point X2;Z2 (Fig. 2). The obtained values for X1, Z1, X2 and Z2 were for temporal and nasal side of each eye entered into a data base. The average data of the five measurements per eye were grouped for normal and keratoconus eyes and statistically described by mean \pm SD. The limbal area, defined as the area between points $\mathrm{X} 1 ; \mathrm{Z} 1$ and $\mathrm{X} 2 ; \mathrm{Z} 2$, was for temporal and nasal side computed for its width and height. The nasal and temporal slopes of the limbal area were expressed by computing the tangent from the width and height values. Resulting data were statistically analyzed for differences using the Student $t$-test.

\subsubsection{Results}

A qualitative analysis of the CSPs revealed that the transition from cornea to sclera is smoother and less pronounced than usually shown in anatomical drawings or as one would expect from a connection between the central corneal and the scleral curvatures. However, all cross sections clearly showed a point (Fig. 2) where the smooth corneal profile changes in a more wave like profile that continues over the sclera. The reproducibility of height data on the smooth corneal portion as analyzed from the five repeated measurements per eye revealed to be very good. The root mean square (RMS) of the height data within a $10 \mathrm{~mm}$ corneal portion was at average $7.95 \mu \mathrm{m}$ (range 1.8 to $13.8 \mu \mathrm{m}$ ) and for the limbal area between 5 to $7 \mathrm{~mm}$ from the center $21.1 \mu \mathrm{m}$ (range 13.7 to $35.8 \mu \mathrm{m}$ ). Typically, on the scleral portion (beyond $7 \mathrm{~mm}$ from the center) the average RMS showed a much higher value 
of $156.8 \mu \mathrm{m}$ (range 52.9 to $291.1 \mu \mathrm{m}$ ). This represents the variation in height topography caused by the loose tissue of the conjunctiva. With the method we used to find the data points $(\mathrm{X} 1, \mathrm{Z1}, \mathrm{X} 2, \mathrm{Z} 2)$ from the five measurements of each eye the reproducibility was within $30 \mu \mathrm{m}$.

Table 1 summarizes the results of the measurements and the analysis of the data. The mean $\mathrm{x}$ values were not significantly different between normal and keratoconus eyes. All z values for temporal and nasal were significantly $(\mathrm{p}<0.05)$ higher in keratoconus eyes compared to normal eyes. The average difference in $\mathrm{z}$ value between normal and keratoconus eyes on all measured points showed a mean of $0.31 \mathrm{~mm}(\mathrm{SD} \pm 0.094)$.

\begin{tabular}{|c|c|c|c|}
\hline Variable & Mean Normal & Mean Keratoconus & $\mathrm{p}$ value \\
\hline X 1 temp & 5.4612 & 5.4960 & .7181 \\
\hline Z 1 temp & 2.0869 & 2.3493 & .0395 * \\
\hline X 2 temp & 6.6249 & 6.7705 & .1126 \\
\hline Z 2 temp & 3.0562 & 3.4261 & .0121 * \\
\hline X 1 nasal & 5.4650 & 5.6045 & .0864 \\
\hline Z 1 nasal & 1.9834 & 2.2630 & .0032 * \\
\hline X 2 nasal & 6.6977 & 6.6528 & .6050 \\
\hline Z 2 nasal & 2.8040 & 3.1462 & $.0049 *$ \\
\hline Tangent temp & 1.2565 & 1.2213 & .7733 \\
\hline Tangent nasal & 1.5911 & 1.2359 & $.0341 *$ \\
\hline Width temp & 1.1637 & 1.2745 & .2498 \\
\hline Width nasal & 1.2329 & 1.0483 & $.0327 *$ \\
\hline Height temp & 0.9693 & 1.0768 & .2892 \\
\hline Height nasal & 0.8206 & 0.8832 & .3998 \\
\hline
\end{tabular}

In normal eyes the mean nasal tangent value was significantly higher than the temporal ( $\mathrm{p}$ $<0.05)$ meaning a lower nasal slope. For keratoconus eyes there was no significant difference between the mean nasal and temporal tangent value. This effect was entirely due to a significantly lower mean nasal tangent value (higher slope) in keratoconus (Fig. 3). We found that the mean temporal tangent value did not vary significantly between normal and keratoconus eyes (Fig. 4). Also the mean width of the nasal limbal area was significantly smaller in keratoconus whereas the mean heights did not differ significantly (Table 1). 


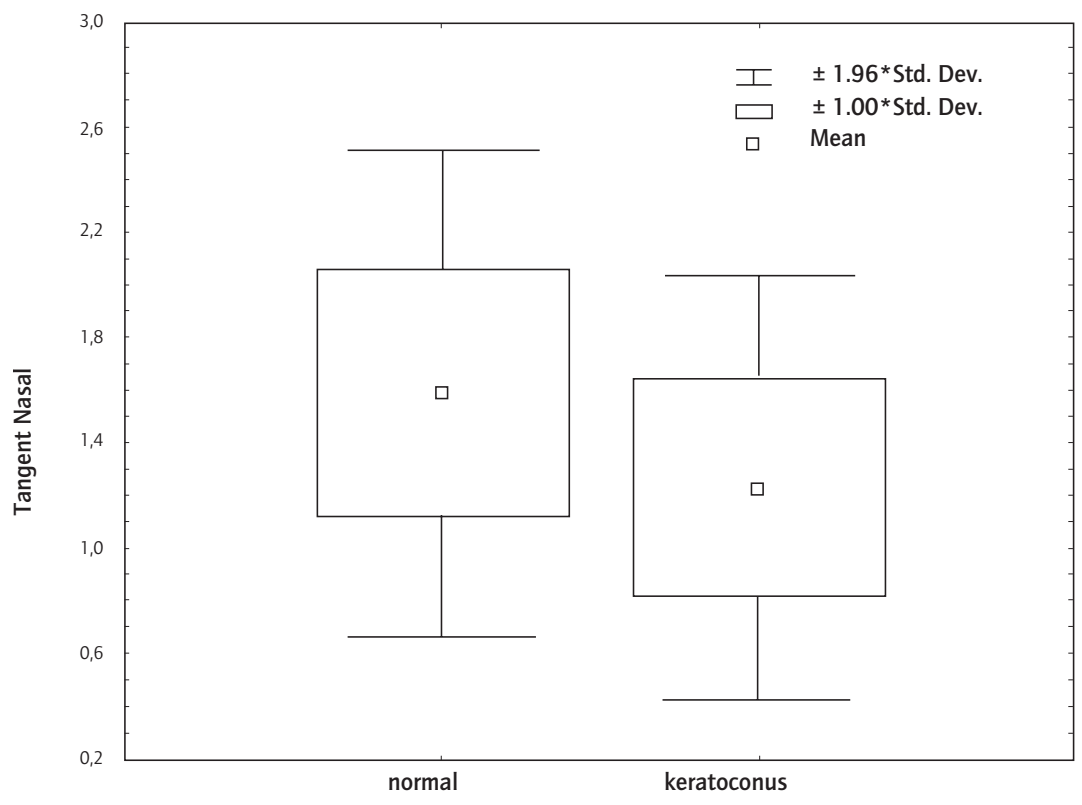

Figure 3. Plot of mean, SD and range of nasal tangent of normal and keratoconus eyes.

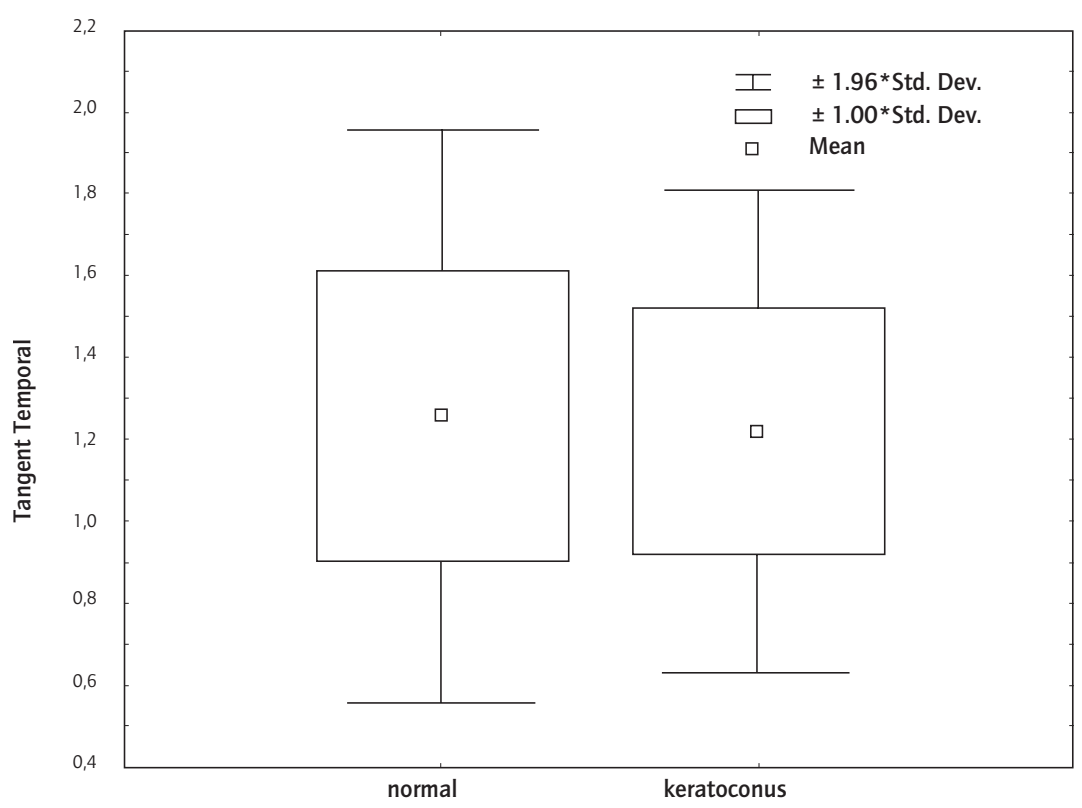

Figure 4. Plot of mean, SD and range of temporal tangent of normal and keratoconus eyes. 


\subsubsection{Discussion}

The method we used to define the limbal area is a first attempt to extract this information from height profiles composed from the MST measurements. The reproducibility of finding the $\mathrm{z}$ and $\mathrm{x}$ values was within $30 \mu \mathrm{m}$ which is fair within the statistical differences we have found between the normal and the keratoconus eyes. The RMS of the measurements with the MST device showed good repeatability on the cornea which were comparable with an earlier study $y^{6}$ in which solid and fixated models of the eye were used. We did not find significant differences in RMS between normal and keratoconus eyes $(p>0.05)$ which means that fixation errors between measurements were low. We must point to the fact that this only means that our measurements were reproducible and that there is no discrepancy between the ability of patients with normal eyes and those with keratoconic eyes to align their visual axis to the fixation target. An entirely different question is the position of the eyes in space relative to the optical axis of the measuring device. This inherent problem in corneal topography as described by Mandell20, is in our case of studying the corneal-scleral profile and comparing normal and keratoconic eyes of particular importance since large differences in fixation via the visual axis can be expected. However, this displacement in keratoconus would particularly involve the vertical meridian whereas we only compared horizontally.

Obtaining non-invasive height profiles of the vertical meridian of the eye was subject to problems. We did some further experiments in gathering images in primary, upward, and downward gaze to compose these to one image. This technique seems to keep tear film disturbance at reasonable levels, but also showed variation in shape mainly at the edges of the eyelids. A software program is now under development to be able to merge the individual images in different directions of gaze into one representing the eye in all meridians.

Classically, the limbus is defined as the junction between cornea and sclera. Since the radii of cornea and sclera differ remarkably this would suggest a sharp transition. Comparing the corneal-scleral profiles in our study with morphological classification as has been suggested by Meier ${ }^{5}$ we found that in most of our profiles the limbal area would be termed as fluent rather than outstanding. However, at some points of the profiles (Fig. 2) the shape could certainly be recognized as convex, tangential or concave. With the fact that the limbus is not one point the classification system using observations of the limbus with the slitlamp seems rather subjective. Our data and a qualitative analysis of the CSPs show agreement with the study of Mandell 4 in which he also found a high variability among eyes and a much less pronounced limbal border than usually assumed.

Our finding that normal eyes show an asymmetry with a lower nasal slope of the limbal area is in accordance with the results of Marriott ${ }^{7}$, who found using the technique of eye impressions that especially in the horizontal meridian a flatter nasal shape is present. Also the work of Pullum ${ }^{2}$, seems with the evaluation of many eye impressions to be in accordance with our findings in normal eyes. The examples of contours of keratoconus eyes given by Pullum show that contrary to normal eyes the horizontal meridian in keratoconus eyes can become more symmetric whereas the vertical meridian becomes more asymmetric. A real (rotational symmetric) conical shape seems to be an exception in keratoconus. Although not quantitatively worked out, these examples show agreement with our finding that in keratoconus 
the horizontal meridian is more symmetric than in normal eyes. A hypothesis on the biomechanical process behind this could be that the corneal limiting membranes (Bowman and Descemet) in keratoconus are softened and subject to major changes in shape extending far beyond the central area ${ }^{8,9}$ including changes in the posterior shape of the cornea. ${ }^{10}$ This hypothesis would also fit with the fact that keratoconus can be recurrent after corneal transplantation. ${ }^{11}$ With the force of intra-ocular pressure this would explain the process that the limbal slopes have a tendency to become equal in keratoconus eyes.

Another question in keratoconus is the position of the cone. We found the temporal limbus to be stable in slope. This is in accordance with the finding of Pullum ${ }^{2}$ that the temporal sclera is near to be tangential to the cornea. Maybe this is the maximum slope the eye can reach on the temporal side. Also, it has been shown that significant thinning of the cornea in keratoconus is present in all quadrants except the far temporal side ${ }^{12}$ and that in keratoconus eyes sensitivity is greatest in the temporal and lowest in the inferior cornea. ${ }^{13}$ Taking these findings together would suggest that the temporal side of the cornea in keratoconus is less affected. On the nasal side the normal eyes in our study showed a lower slope than the keratoconus eyes. With the "weakening structure hypothesis under intra ocular pressure" as stated above, there might be room for the corneal mass to extent its increase in $\mathrm{z}$ value into a displacement of its top. Assuming from the above, that the temporal corneal tissue remains stable this would biomechanically suggest a nasal displacement of the corneal top. In our study on 11 keratoconus eyes we found the top of the cornea to be displaced in infero-temporal direction in 7 cases (64\%), and in infero-nasal direction in 4 cases (36\%). In a study by Auffart, et al. ${ }^{14}$ using a height measuring corneal topography system (Orbscan) inferiortemporal displacement was found in 68 out of 71 cases. Demirbas and Pflugfelder ${ }^{15}$ using a system based on raster stereoscopy (PAR) found $65 \%$ of the cases to be displaced inferotemporal. Zabala and Archila ${ }^{13}$ using a topogometer reported that marked corneal steepening was found inferior-temporal in 59\% of the cases. Although there seems to be evidence that an infero-temporal displacement of the corneal top is present in around $60 \%$ of the keratoconus eyes, the remaining $40 \%$ of the cases in which the displacement varies considerably bears a problem to come to a universal explanation of the biomechanical changes connected to the disease. Maybe the penetration of weakening factors along the limiting membranes of the cornea vary in keratoconus not only per eye but also per semi meridian, and consequently the possible changes in shape do vary with ocular rigidity ${ }^{16}$ from case to case. In discussing the possible mechanical forces behind the shape of keratoconus eyes we must bear in mind that keratoconus eyes in the majority of the cases have been fitted with rigid contact lenses that may induce so-called corneal warpage. Therefor, the principle question is whether the shapes as we and other authors have measured or observed are due to the keratoconus or due to the influence of the contact lens on it. ${ }^{17}$ An interesting study in this respect is done by Rabinowitz and McDonnell. ${ }^{18}$ They found that keratoconus eyes that had not worn contact lenses for at least one year prior to examination commonly show an inferior steepening more prominent temporally, and central steepening with a superimposed asymmetric bowtie pattern. These findings are contrary to ours and those using profiles from casts in which it seems that the temporal side of the cornea in keratoconus remains stable. However, the difference in measuring method is also evident. Placido CAVK is limited 
to a more central part of the cornea and the analysis is very sensitive to local slope, whereas profilometry involves the analysis of height data over the entire surface of the cornea and sclera. Smolek and Klyce ${ }^{19}$ using CAVK concluded from surface area measurements (120 $\mathrm{mm}^{2}$ ) that keratoconus, with the exception of keratoglobus, is not a true ectasia but rather a special type of corneal warpage. Our data on the entire cornea suggest the opposite since all $\mathrm{z}$ values were significantly higher in keratoconus eyes. To better compare these two studies we have calculated the differences in $\mathrm{z}$ values between normal and keratoconus eyes at points $3 \mathrm{~mm}$ from the center. The results revealed an average z value of $0.68 \mathrm{~mm}$ (range 0.57 to $0.82 \mathrm{~mm}$ ) for normal eyes and an average of $0.76 \mathrm{~mm}$ (range 0.62 to $1.03 \mathrm{~mm}$ ) for keratoconus eyes. The difference is not statistically significant $(p>0.05)$. It seems that the topographical changes in keratoconus eyes as measured with profilometry indicate higher $\mathrm{z}$ values overall and more symmetrical slopes of the nasal and temporal limbal areas. In the central area of the cornea both CAVK and profilometry indicate large variations in warpage effects. These findings support the theory of biomechanical coupling as has been suggested by Smolek and Klyce. ${ }^{19}$

\subsubsection{Conclusion}

With the MST topographer, height topography of the whole visible horizontal ocular surface could be obtained. The limbal area could be detected and measured horizontally.

Keratoconus eyes had a significantly (mean $0.31 \mathrm{~mm}$ ) higher $\mathrm{z}$ value.

In normal eyes the mean nasal limbal slope was significantly lower than the temporal. In keratoconus eyes this difference was not present. It can be hypothesized that in keratoconus eyes the nasal limbal area has become steeper. It is debated whether these changes are due to the development of the keratoconus or due to the influence of contact lens wear.

This study supports the hypothesis of the existence of a biomechanical coupling over the entire corneal surface that in keratoconus eyes leads to more symmetric limbal slopes, but also to yet unexplained diverse warpage of the cornea more centrally. Further study including more patients at various stages of keratoconus is needed to gain an inside of the processes behind geometrical changes of the cornea in these cases.

The $\mathrm{x}, \mathrm{y}, \mathrm{z}$ co-ordinates of the measured points can, with a tear film design interface, be used as input to micro precision lathing of CLs.

\section{Acknowledgment}

This study was part of the BRITE-EURAM project BE96-4608, Contact Lenses with Aspheric Asymmetric Surfaces (CLAAS) and supported by a grant from the European Commission. 


\section{References}

1. Woodward EG. Preformed scleral lens fitting techniques. Contact Lenses, 3 ed. Edited by Phillips AJ, Stone J. London, Butterworths, 1989.

2. Pullum KW. Eye impressions, production and fitting of scleral lenses and patient management. Contact Lenses, 3 ed. Edited by Phillips A, Stone J. London, Butterworths, 1989.

3. Dallos J. The individual fitting of contact glasses. Trans Ophthalmol Soc 1937;57:509-20.

4. Mandell R. Morphometry of the Human Cornea, Thesis, Indiana University, 1962.

5. Meier D. Das Cornea-Skleral- Profil - ein Kriterium individueller Kontaktlinsenanpassung. Kontaktlinse 1992:4-11.

6. Jongsma FH, de Brabander J, Hendrikse F, Stultiens BA. Development of a wide field height eye topographer: validation on models of the anterior eye surface. Optom Vis Sci 1998;75:69-77.

7. Marriott PJ. An analysis of the global contours and haptic contact lens fitting. Br J Physiol Opt 1966;23:1-40

8. Edmund C. Corneal topography and elasticity in normal and keratoconic eyes. A methodological study concerning the pathogenesis of keratoconus. Acta Ophthalmol Suppl 1989;193:1-36.

9. Wilson SE, Lin DT, Klyce SD. Corneal topography of keratoconus. Cornea 1991;10;1:2-8.

10. Tomidokoro A, Oshika T, Amano S, Higaki S, Maeda N, Miyata K. Changes in anterior and posterior corneal curvatures in keratoconus. Ophthalmology 2000;107;7:1328-32.

11. Bechrakis N, Blom ML, Stark WJ, Green WR. Recurrent keratoconus. Cornea 1994;13:73-7.

12. Owens $\mathrm{H}$, Watters GA. An evaluation of the keratoconic cornea using computerised corneal mapping and ultrasonic measurements of corneal thickness. Ophthalmic Physiol Opt 1996;16;2:115-23.

13. Zabala M, Archila EA. Corneal sensitivity and topogometry in keratoconus. CLAO J 1988;14;4:2102.

14. Auffarth GU, Wang L, Volcker HE. Keratoconus evaluation using the Orbscan Topography System. J Cataract Refract Surg 2000;26;2:222-8.

15. Demirbas NH, Pflugfelder SC. Topographic pattern and apex location of keratoconus on elevation topography maps. Cornea 1998;17;5:476-84.

16. Foster CS, Yamamoto GK. Ocular rigidity in keratoconus. Am J Ophthalmol 1978;86:802-6.

17. Szczotka LB, Rabinowitz YS, Yang H. Influence of contact lens wear on the corneal topography of keratoconus. CLAO J 1996;22;4:270-3.

18. Rabinowitz YS, McDonnell PJ. Computer-assisted corneal topography in keratoconus. Refract Corneal Surg 1989;5;6:400-8.

19. Smolek MK, Klyce SD. Is keratoconus a true ectasia? An evaluation of corneal surface area. Arch Ophthalmol 2000;118;9:1179-86.

20. Mandell RB. The Enigma of the Corneal Contour. CLAO J 1992;18:267-73. 


\subsection{Pupillometry}

Infrared Pupillometry in Presbyopes Fitted with Soft Contact Lenses

Nicolas Chateau ${ }^{1}$, John de Brabander ${ }^{2}$, Florence Bouchard ${ }^{1}$ and Henk Molenaar ${ }^{3}$.

From:

1 Ocular Sciences (formerly Essilor CL division), R\&D International, Créteil, France.

2 Academic Medical Center, University of Amsterdam, the Netherlands.

3 Ocular Sciences (formerly Lunelle) 's-Hertogenbosch, the Netherlands.

Optom Vis Sci 1996;73;12:733-41. 
Abstract

Purpose. The influence of pupil size, lens centration, and translation on multifocal contact lens performance suggests that these parameters should be accounted for in the optical design of presbyopic contact lenses. The purpose of the present article is to complete the existing experimental data with clinical measurements of pupil diameter and lens position relative to the pupil in a group of presbyopes, with controlled target luminance, stimulus distance, gaze angle and convergence.

Methods. The experiments involve an infrared video-pupillometer and ring-marked contact lenses.

Results. Results on pupil aperture show that the effect of changing target distance is comparable in magnitude to that of varying luminance within the photopic range. The measured data on lens position confirm that soft contact lenses are generally decentered from the pupillary axis, usually in inferior temporal direction, and that their average translation between primary and downward gaze is very small. It is also found that the average relative position between lens and pupil centers is slightly modified by changes of luminance in far vision.

Conclusion. All these results provide characteristic values which are helpful for the optical modeling of presbyopic contact lenses.

Key words:

Presbyopia, pupil, soft contact lenses, multifocal contact lenses. 


\subsubsection{Introduction}

The visual performance of multifocal contact lenses is known to depend on various factors. ${ }^{1}$ Several studies ${ }^{1-5}$ have pointed out the strong influence of highly subject-dependent parameters, such as pupil diameter, lens position in relation to the pupil, and lens on-eye translation with changing gaze direction. In the design of presbyopic lenses, it appears necessary to take these three relevant factors into account, in addition to more commonly considered parameters, such as the required additive power of the lens. The theoretical modeling of multifocal lens performance could be improved by introducing into the optical calculations the values of these three parameters which can typically be found in the presbyopic population. The variability components of these factors, relative to changing visual conditions and to intersubject differences, could also help to specify tolerance requirements for multifocal designs. Our interest in this field has motivated the present study, with the objective of bringing a useful description of the distributions of pupil size, lens centration and translation in the presbyopic population.

\section{Pupil diameter}

Pupil size is a key parameter to the visual performance of most multifocal lenses ${ }^{1-5}$ : for instance, with a refractive bifocal lens, pupil diameter directly determines the proportion of light refracted by each corrective zone. It has been shown that the pupil diameter varies with aging, becoming steadily smaller from adolescence until about age of 60.6-9 The amplitude of the pupil response to light also slowly decreases with aging. ${ }^{6}$ Although it also belongs to common knowledge that myopes generally have larger pupil apertures than hyperopes, no clinical evidence of such a difference was established in presbyopes. ${ }^{10}$ The clinical results on pupil size always exhibit a strong inter-individual variability; the pupil diameter of one individual is also highly dependent on environmental conditions, and on individual factors such as concentration, fatigue and emotion. ${ }^{9}$ It is thus reasonable to consider that the pupil diameter in the presbyopic population can not be described by a unique typical value which would be representative for all conditions: at least the influence of light and target distance should be considered. The adaptation of pupil size to changing luminance has been extensively studied, ${ }^{9-12}$ but the influence of stimulus distance has seldom been considered, and very little work on the pupil in specific visual conditions has been specifically carried out with presbyopes. The combined effects of target proximity and luminance on pupil size were measured by Schäfer and Weale ${ }^{12}$ in four age groups, including a group of presbyopes. Their results give an interesting description of the miosis induced by near vision, which appears as an important phenomenon at all ages. However, the pupil diameter values reported in this reference ${ }^{12}$ can not be considered as typical of presbyopes in normal viewing or reading conditions, since this study involved very few presbyopes and was made in special laboratory conditions: the eye was illuminated by a flash lamp, and accommodation was stimulated by a small movable target without readable characters. More characteristic and realistic results could be obtained in a larger population of presbyopes, with reading targets of more common size, and using a less disturbing apparatus. 


\section{Lens centration}

A decentered multifocal lens may exhibit unexpected visual performance, ${ }^{2-5}$ since decentration modifies the relative proportions of corrective zone areas which intersect the pupil. In the common fitting practice, the centration of soft contact lenses is estimated in relation to the limbic region with the help of a biomicroscope; consequently, to our knowledge, very few published papers report values of lens position relative to the pupil. Erickson and Robboy ${ }^{13}$ used a slit lamp with special eyepiece reticule to make such measurements in 15 presbyopes fitted with soft contact lenses. The precision of decentration evaluation is not given in their paper. Their results exhibit a tendency of the optical zone centers to be shifted of typically $1 \mathrm{~mm}$ in the inferior temporal quadrant of the pupil. With a similar method, Woods et al. ${ }^{14}$ obtained decentration measures of rigid lenses in 5 presbyopes, with mean value slightly higher than $1 \mathrm{~mm}$. The subject convergence and gaze angles during this experiment was not reported. Since these parameters affect the relative position between eye and lids, they might have an influence on lens centration : such effects of lens translation are discussed further in this introduction. Lens centration relative to the pupil may also be modified by luminance-induced changes in the centration of the pupil itself, since at least two studies revealed significant measured shifts of the pupil center with changing pupil diameter. ${ }^{15,16}$ In most cases, these movements occur in temporal direction on pupil dilation, and remain limited to a few tenths of millimeters. ${ }^{16}$

\section{Lens translation}

A change in the mean on-eye position of the contact lens may occur when the eye switches from primary to downward gaze. Such a lowering of the line of sight most usually happens when one changes from distant observation to a reading posture, which is also generally associated with an increased convergence. When the eye rotates downward, the inferior lid is believed to exert an action which tends to hold back the lens movement and results in its upward on-eye translation. ${ }^{4,17}$ Such a translation may affect multifocal lens optical performance, since it modifies the centration of the lens relatively to the pupil. This effect is actually used as a basic mechanism in alternated vision contact lenses. In order to verify the feasibility of such lenses, the amplitude of the inferior lid action with lowering gaze direction was clinically evaluated by Borish and Perrigin ${ }^{18}$ : they measured the differences of movement of line of sight and lower lid on 107 subjects without contact lenses. As the average relative shift was found to be of $0.8 \pm 0.35 \mathrm{~mm}$, one may predict a poor ability of the lower lid to induce contact lens translation, especially in the case of soft lenses. Indeed, other slit lamp measurements ${ }^{13}$ exhibited a mean soft contact lens translation of $0.4 \pm 0.7 \mathrm{~mm}$ in $30^{\circ}$ downgaze. It is likely that this value was averaged on scalar translation lengths, rather than on translation vectors : such a summation tends to overestimate the mean translation length. For multifocal design, it would be more interesting to know the lens translation vectors between distant viewing and near reading, measured on presbyopes with at least two different controlled gaze angles.

From the preceding review, it appears that we need more clinical data on pupil size, lens centration and translation in presbyopes, measured with controlled conditions of luminance, target distance and gaze angle; these experiments should be designed in order to exert on 
subjects a reading stimulation as natural as possible, and to avoid perturbing them with the measuring apparatus. In order to fulfill these experimental requirements, we built a set-up which includes an infrared pupillometer and specially marked contact lenses, as described further in the paper. We used this equipment in a clinical study on a group of presbyopes, with the purpose of partially compensating for the lack of available data of practical interest for the design of multifocal contact lenses.

\subsubsection{Materials and Methods}

\section{Pupillometer}

In our video-pupillometer apparatus, as depicted in Figure 1, the stimulus target is viewed through a half-reflecting plate located close to the subject's eyes. A chin-rest maintains the subject's head behind the plate, which is large enough to avoid perturbing vision by excessive restriction of the field of view. The half-reflecting plate is oriented so that the light emitted by infrared LEDs is redirected onto the subject's eye, and that an image of the eye is reflected onto a CCD camera. The illumination system and the camera are surrounded by a dark background which helps to make them invisible to the subject during the measurement. A framegrabber connected to the camera allows quick image recording on a computer hard disk. The upper part of the set-up, which holds the camera and the LEDs, can be rotated around an axis which roughly correspond to the imaginary line joining the centers of subject's eyes. This feature allows to keep the image of the eye in-focus and well centered when the subject changes her/his gaze direction in a vertical plane. Yet, the apparatus can not be adjusted for the convergence angle : this results in a small artifact in the measurement of horizontal lengths. For instance, if a subject converges on a stimulus located $40 \mathrm{~cm}$ in
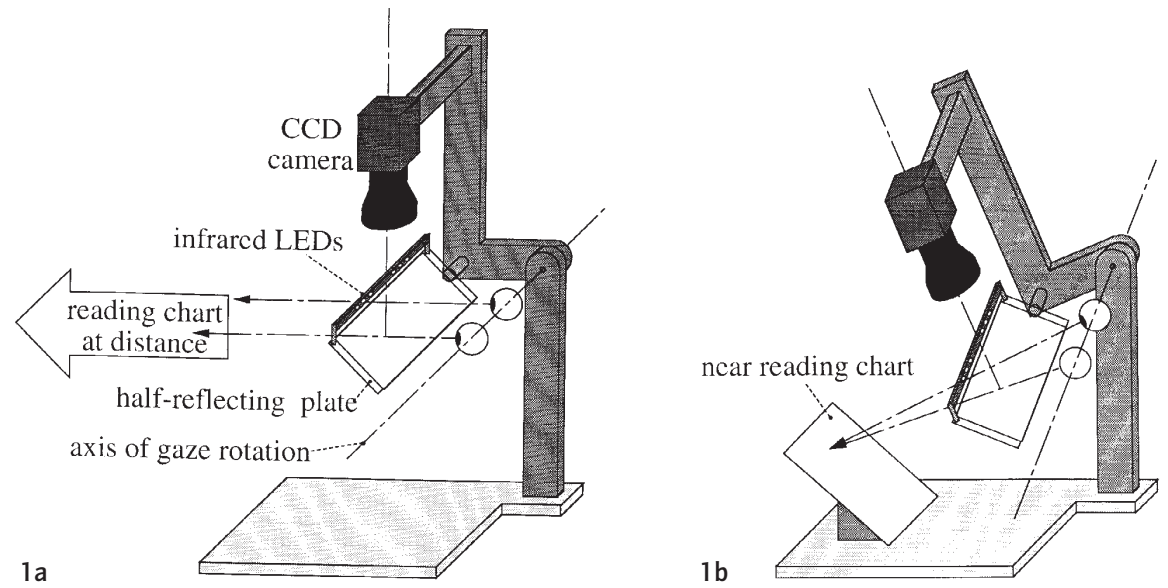

Figure 1. Scheme of the experimental infrared video-pupillometer apparatus configured for measurement with subject in primary gaze (a) and in downward gaze (b) (for simplicity, the chin-rest is not shown and only the subject's eyes are depicted. 
front of him, the apparent pupil is approximately $8 \%$ larger than the real one in the horizontal direction. This is easily compensated by applying the inverse horizontal magnification. In Figure 1, the apparatus is represented in two different orientations which correspond to measurements in primary (Fig. 1a) and downward gaze (Fig. 1b).

\section{Ring-marked contact lenses}

Subjects are asked to read in several different conditions of stimulus proximity and luminance, while repeated images of their eyes are recorded for further analysis of pupil size and lens position. In an image of the eye wearing a standard soft contact lens, the location of the lens center -or optical axis- can be estimated from the position of the lens edges. The precision of such a method is relatively poor with our infrared images, because the lens edge is usually difficult to detect and is partially hidden by the lids. In order to obtain easier and more accurate measurement of lens centration, we printed a white ring mark of $10 \mathrm{~mm}$ diameter onto the contact lenses. The ring diameter is large enough for the circle not to be noticed by presbyopic wearers. A picture of the eye wearing a ring-marked lens is presented in Figure 2.

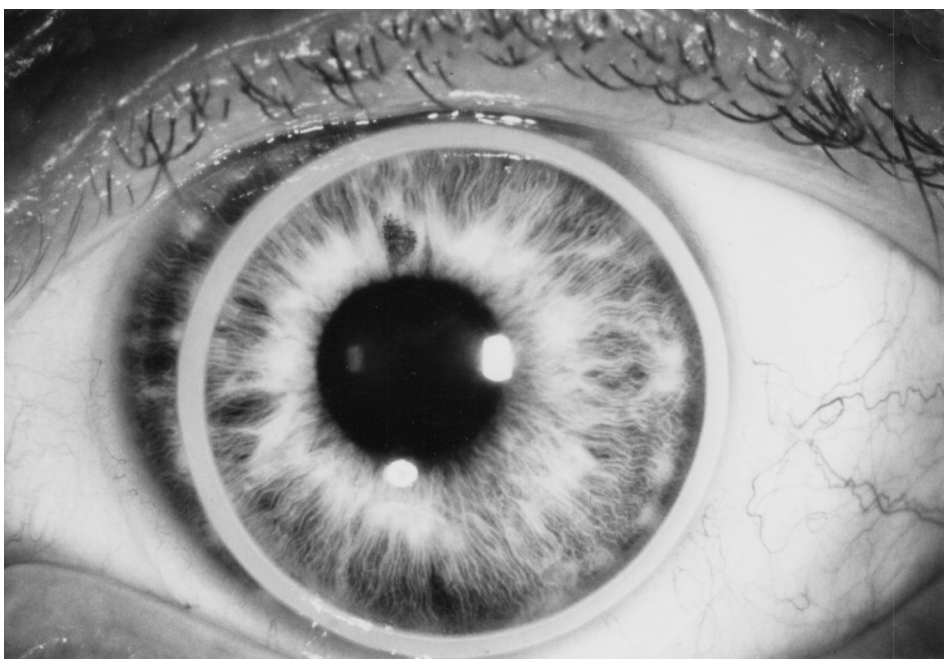

Figure 2. Ring-marked soft contact lens on the eye.

Lenses are highly hydrophilic (70\% water content) monofocal lenses, with monocurve posterior geometry, diameter $14 \mathrm{~mm}$ and center thickness $0.2 \mathrm{~mm}$. The available power range lies between -6 to $+6 \mathrm{D}$ (step $0.5 \mathrm{D}$ ), and base curve radius (BCR) can be chosen between 8.3 and $9.2 \mathrm{~mm}$ (step $0.3 \mathrm{~mm}$ ). As a fitting rule, the BCR of the first trial lens is deduced from the average keratometric radius $\mathrm{K}_{\mathrm{m}}$ with the formula: $\mathrm{BCR}=\mathrm{K}_{\mathrm{m}}+0.8 \mathrm{~mm}$. If necessary, subsequent lens trials with different BCR values are carried out, until both movement and centration are rated as acceptable under biomicroscopic observation. ${ }^{19}$

Monofocal, rather than multifocal contact lenses are used in our experiments in order to 
ensure more natural visual conditions. Otherwise, pupil diameter results could be influenced by visual effects related to the particular multifocal optics under test, and could not be considered as standard values. As the typical difference between the surfaces of common monofocal and refractive multifocal contact lenses with same distance correction is about $20 \mu \mathrm{m}$ ( 1 tenth of total lens thickness), we can estimate that the centration and translation characteristics of multifocal lenses are very close to those obtained with monofocal lenses.

\section{Image analysis}

Each image is analyzed with a computer software which displays two circles graphically superimposed to the eye: the user modifies the circle diameters and center positions, in order to fit one circle to the edge of the pupil, and the other one to the lens ring mark. The resulting circle parameters are used by the program, in combination with calculated magnification factors of contact lenses, trial spectacles lenses and camera, to compute the pupil diameter and the relative position between the lens optical axis and the center of the pupil. This analysis assumes that the human pupil has a circular edge: as our main objective is to determine typical pupil size for the modeling of multifocal lenses, we consider that any ellipticity can be neglected as a secondary order factor.

It must be noted that the preceding methods could not be used to measure lens position in relation to the visible iris without several modifications: the iris edge is too often hidden by the ring mark, and its contrast in the infrared image is usually very low. The described apparatus is convenient for measuring both pupil size and lens decentration relative to the pupil within a very short time.

\section{Measuring conditions}

Two different reading targets are used as far and near vision stimuli. Vision at distance is stimulated by a letter chart with controlled luminance, situated at $4.5 \mathrm{~m}$ from the subject's eyes. The vertical positioning of the chart is adjusted in order to make the line of sight horizontal in distance vision. For measurements in near vision, the subject is asked to read a Parinaud-Weiss chart, illuminated by two halogen projectors with adjustable intensity. The near acuity chart is located $40 \mathrm{~cm}$ in front of the eyes, and compels the subject to read in downgaze, with an angle of $23^{\circ}$ to the horizontal. The room contains no other light sources than those used for stimulus illumination. In both targets, only letters corresponding to Snellen acuity $>0.7$ (20/30) are used, in order to achieve maximal stimulation of accommodation-related mechanisms. Two values of photopic luminance are presented to the subject: low luminance $\left(\mathrm{L}_{\mathrm{L}}\right)=50 \mathrm{~cd} \mathrm{~m}^{-2}$ and high luminance $\left(\mathrm{L}_{\mathrm{H}}\right)=350 \mathrm{~cd} \mathrm{~m}^{-2}$ (values corrected from the loss due to the half reflecting plate). According to preliminary experiments, where 15 presbyopic subjects were asked to adjust target luminance and to scan the range which allowed them to read, luminances $\mathrm{L}_{\mathrm{L}}$ and $\mathrm{L}_{\mathrm{H}}$ roughly represent the limits of the range considered as comfortable. ${ }^{20}$

\section{Clinical protocol}

We enrolled 112 subjects who satisfied to the following criteria: age $\geq 45$, need for spherical correction $\leq 6 \mathrm{D}$, cylinder correction $\leq 0.75 \mathrm{D}$, near vision addition $>0.75 \mathrm{D}$, normal 
ocular condition, no uncompensated heterophoria. Each subject was fitted with a pair of ring-marked contact lenses and, when necessary, was provided with complementary spherical trial frame corrections adapted to each target distance. The lenses were allowed to settle during 15 minutes. Each eye was then measured in 4 different experimental conditions (2 distances $\mathrm{x} 2$ luminances). When luminance was modified, an adaptation period of 5 minutes was respected. The investigators waited for a delay of approximately 2 seconds after blinking before capturing each image of the eye. In each experimental configuration (distance / luminance), 2 repeated images were taken. Preliminary trials have shown that averaging the data of 2 images obtained in the same conditions generally results in an accuracy of $\pm 0.2 \mathrm{~mm}$ on both pupil diameter and lens decentration (95\% confidence interval). In our clinical method, a total of 16 pupillometer images was obtained for each subject. The study protocol was approved by the ethical committee of the Academic Medical Center of Amsterdam.

\section{Statistical analysis}

It is already known that shortening target distance or increasing luminance results in a smaller pupil aperture. We intended to confirm these results with our data through a multiway analysis of variance (ANOVA). In order to account for the measuring error, the test included all data from repeated measurements. In summary, the ANOVA followed a 2 (repeated factor: distance) x 2 (repeated factor: luminance) x 2 (repeated factor: order of measurement) design. The relations between pupil size and respectively age and ametropia in the presbyopic population were also checked by multiple regressions in all 4 experimental conditions.

The significance of average decentration results was evaluated by checking confidence interval. In near vision, we anticipated that downgaze would result in a higher position of the lenses, and that increased convergence might induce a displacement of the lenses in the temporal direction; these effects of target distance (e.g. gaze angle and convergence) on repectively the horizontal and vertical components of lens decentration were tested through two separate ANOVAs. The design of these ANOVAs was: 2 (repeated factor: distance) x 2 (repeated factor: luminance) x 2 (repeated factor: order of measurement) x 4 (independent factor: BCR).

\subsubsection{Results}

Four subjects could not be succesfully fitted with the ring-marked soft contact lenses. Our results thus involve data from 108 subjects. The distributions of age and ametropia (sphere equivalent refractive error) in these subjects are represented respectively in Figures 3 and 4. The BCR distribution of the experimented lenses is given in Table 1.

Table 1. Base curve radii of the contact lenses fitted in the study.
\begin{tabular}{llllll}
\hline BCR $(\mathrm{mm})$ & Excluded & 8.3 & 8.6 & 8.9 & 9.2 \\
Number of eyes & 8 & $13(6.0 \%)$ & $89(41.2 \%)$ & $107(49.5 \%)$ & $7(3.3 \%)$ \\
\hline
\end{tabular}




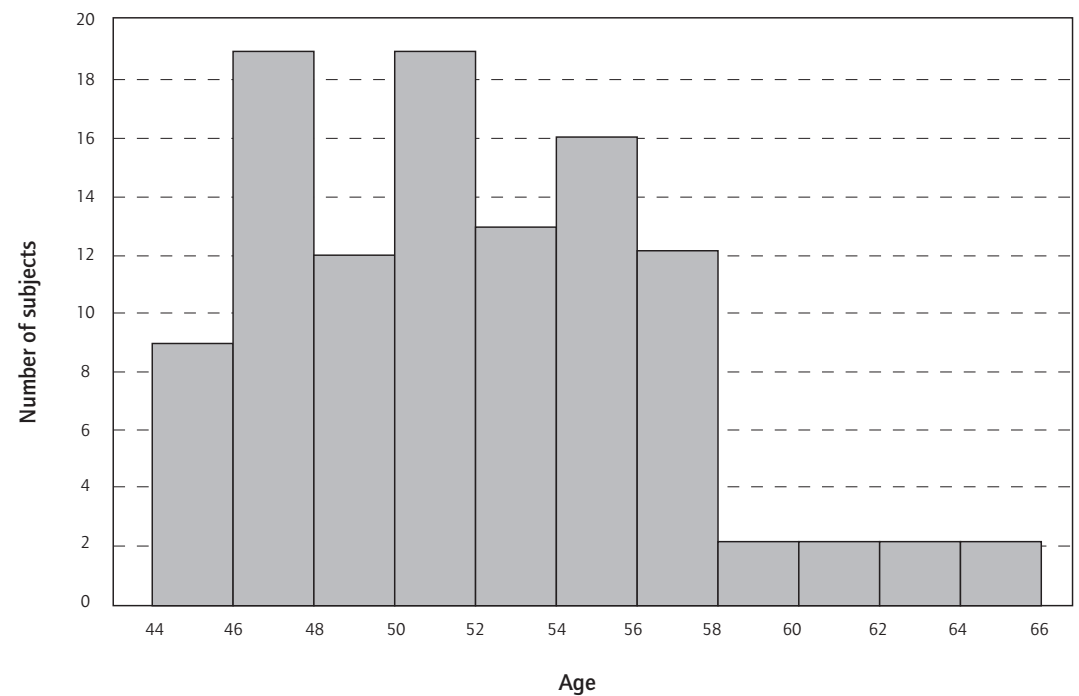

Figure 3. Distribution of the age of presbyopic subjects.

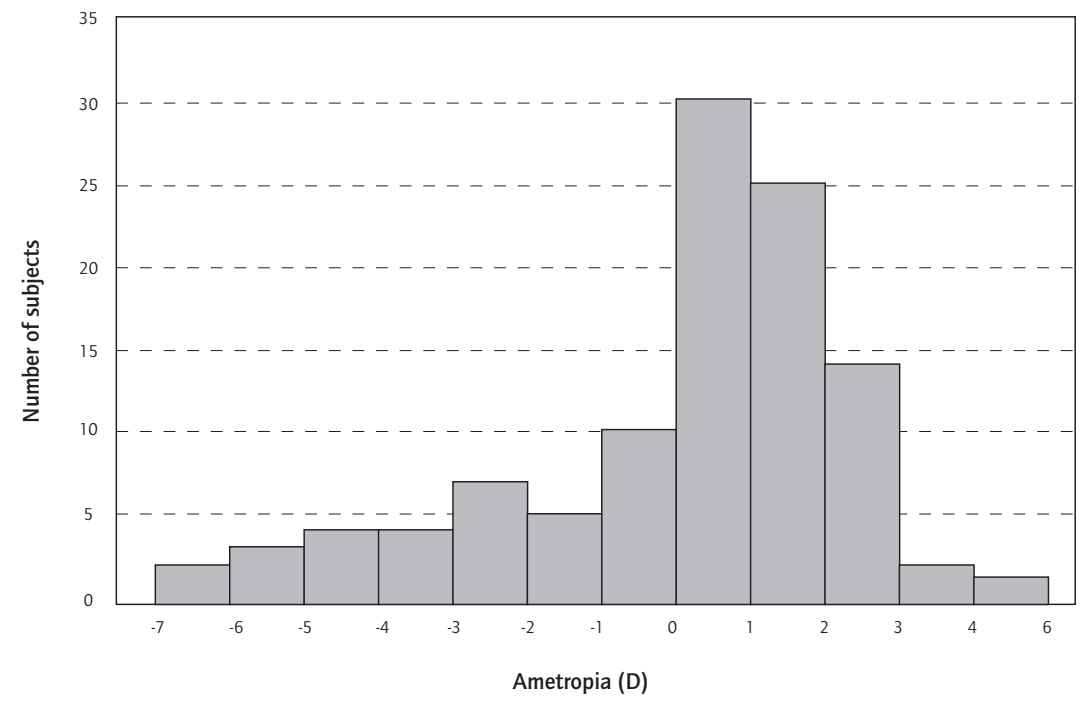

Figure 4. Distribution of ametropia, represented by sphere equivalent refractive error.

\section{Pupil diameter}

The mean values and standard deviations of pupil size are given in Table 2. One can easily compare the effects of changing luminance and changing target distance, which are both highly significant $(p<0.001)$ : in our measuring conditions, the pupil constriction related to increasing luminance has a mean amplitude of $1.0 \mathrm{~mm}$ in far vision, and of $0.6 \mathrm{~mm}$ in near 
Table 2. Mean and standard deviation values of pupil diameter measured in distance and near vision with two different photopic luminances.

\section{$L_{L}=50 \mathrm{cdm}^{-2}=350 \mathrm{cdm}^{-2}$}

$\begin{array}{lll}\text { Distance vision } & \text { Diam. }=4.7 \pm 0.85 \mathrm{~mm} & \text { Diam. }=3.7 \pm 0.60 \mathrm{~mm} \\ \text { Near vision } & \text { Diam. }=3.3 \pm 0.61 \mathrm{~mm} & \text { Diam. }=2.7 \pm 0.42 \mathrm{~mm}\end{array}$

vision; the near vision miosis corresponds to a decrease in diameter of $1.4 \mathrm{~mm}$ with luminance $\mathrm{L}_{\mathrm{L}}$, and of $1.0 \mathrm{~mm}$ with luminance $\mathrm{L}_{\mathrm{H}}$. In Table 2 , it can also be noticed that the inter-individual variability of pupil size is stronger under the experimental conditions which induce larger average diameters.

We find no significant partial correlation between ametropia and pupil size. Linear regression revealed low yet significant apparent correlations between age and pupil diameter $(\mathrm{p}<0.05$ under all different experimental configurations). Regression lines of figure 5 illustrate the time rate of this effect with the following equations: $\phi=6.7-0.038 \times$ age $\left(\mathrm{r}^{2}=0.18\right)$ in far vision and luminance $\mathrm{L}_{\mathrm{L}}, \phi=4.9-0.023 x$ age $\left(\mathrm{r}^{2}=0.10\right)$ in far vision and luminance $\mathrm{L}_{\mathrm{H}}, \phi=4.3-0.018 \times$ age $\left(\mathrm{r}^{2}=0.09\right)$ in near vision and luminance $\mathrm{L}_{\mathrm{L}}, \phi=3.8-0.019 x$ age $\left(\mathrm{r}^{2}=0.11\right)$ in near vision and luminance $\mathrm{L}_{\mathrm{H}}$.

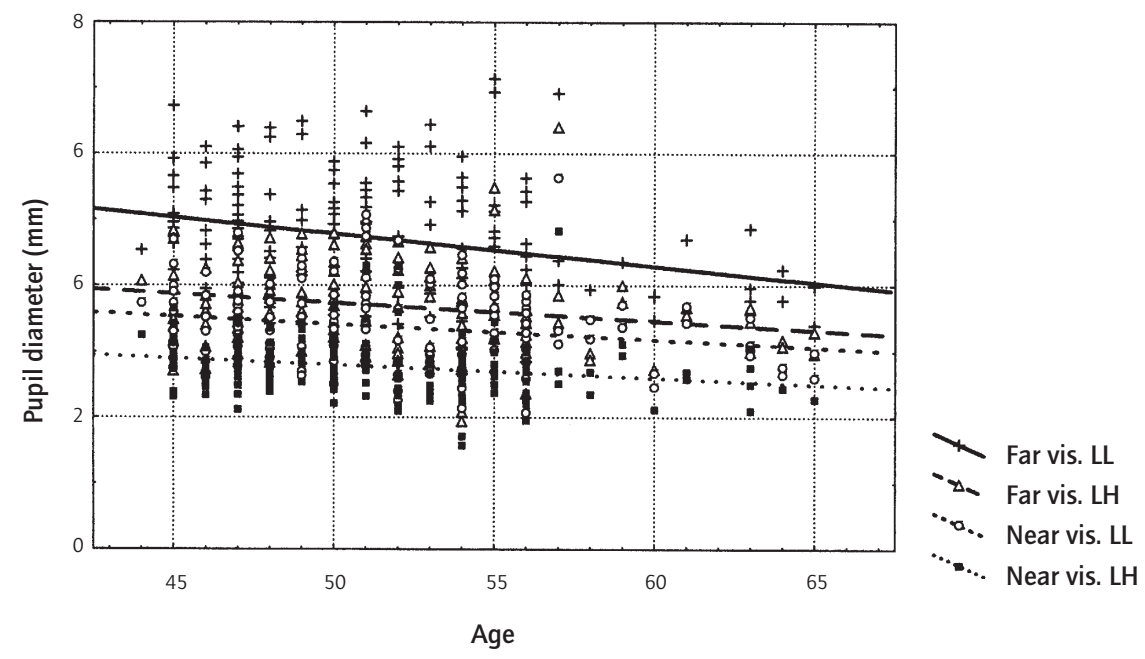

Figure 5. Representation of measured pupil diameters with respect to age, in all 4 experimental configurations; each value of diameter is an average of two measurements. The pupil size is expressed in millimeters and the age in years $\left(L_{L}=\right.$ Low Luminance; $L_{H}=$ High Luminance). 


\section{Lens centration}

The results of lens centration in relation to the pupil are represented in Figure 6. In all 4 experimental configurations, although inter-individual variability is high, the lens centers appear to be almost systematically shifted in the inferior temporal quadrant of the pupil. All the average values of decentration components are significantly different from $0(\mathrm{p}<0.001)$. The mean values and standard deviations of horizontal decentration are given in Table 3. These results suggest that horizontal decentration is related to the luminance level, especially in far vision where lower luminance yields smaller horizontal decentration, and that its mean value does not depend on target distance (we recall that, in our experiments, gaze angle and convergence are determined by the choice of target distance). The ANOVA reveals highly significant effects of luminance, and interaction between luminance and target distance $(\mathrm{p}<0.001)$. Further post-hoc analysis confirmed that luminance significantly influences decentration in far vision $(\mathrm{p}<0.001)$, but not in near vision. We did not detect any significant effect related to the chosen BCR.

Superior

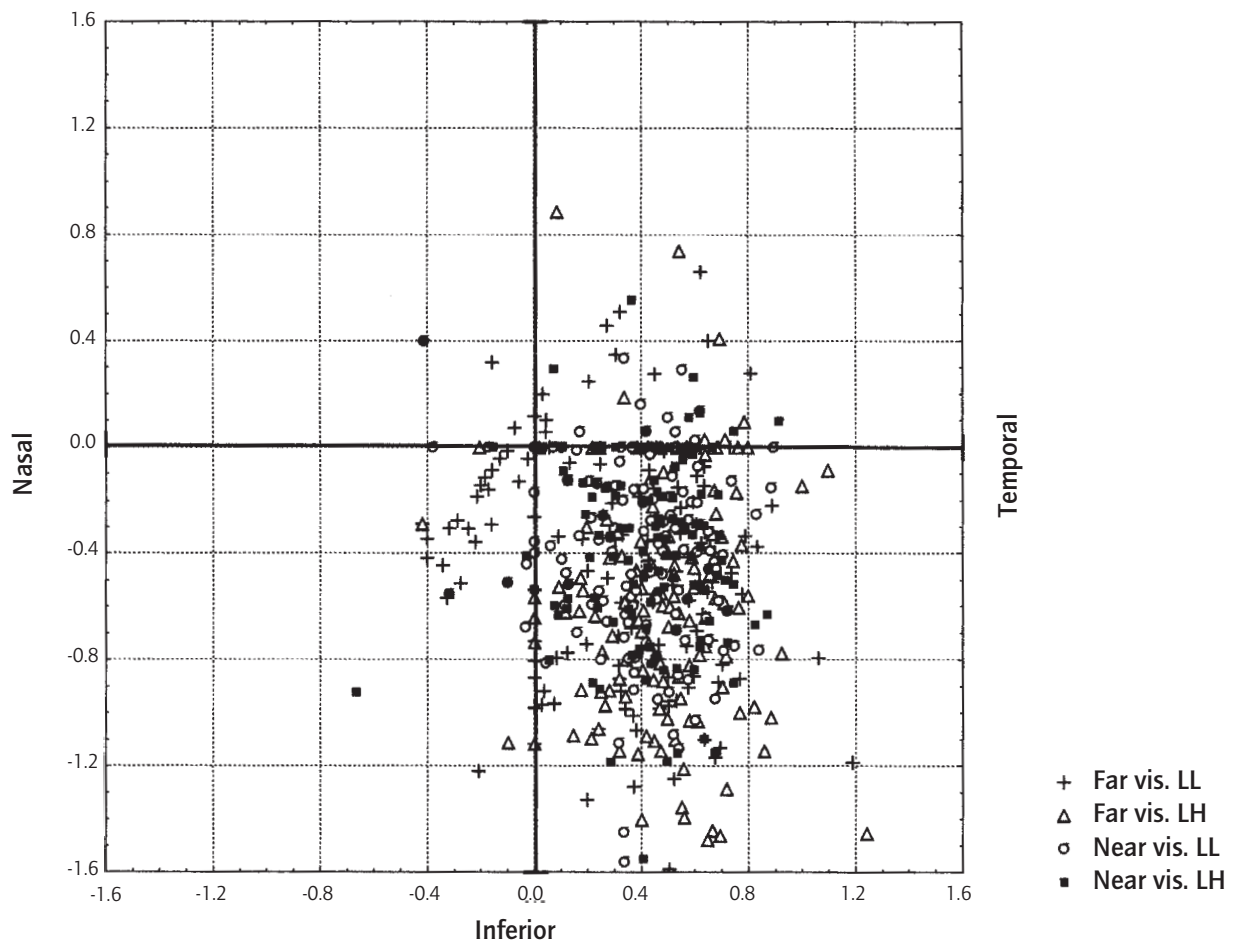

Figure 6. Geometrical representation of measured lens position in relation to the pupil, in all 4 experimental configurations. The graph origin corresponds to the pupil center; each marker symbolizes the center location of one contact lens (average of two measurements). 
Along a vertical axis, we observe a slight improvement of centration relative to the pupil in near vision, as compared to distance vision (see Table 4). The statistical analysis confirms that the mean vertical component of decentration is very significantly influenced by target distance, and also by luminance $(\mathrm{p}<0.001)$. Again, post-hoc testing reveals that pupil size differences due to changes in luminance are significant only in far vision $(\mathrm{p}<0.001)$ : vertical decentration appears to be smaller with lower luminance. We observed no significant influence of the BCR on decentration.

Mean results on vector relative position between lens and pupil centers are summarized in Figure 7. Depending on experimental conditions, mean vector lengths are comprised between 0.5 and $0.8 \mathrm{~mm}$, and mean angles between vectors and horizontal axis lie between $45^{\circ}$ and $53^{\circ}$. As we previously observed on separate coordinates, lens decentration clearly appears to be affected by changes in target distance (and corresponding gaze angle), and by variations of luminance in far vision.

\section{Lens translation}

Vector averages of these translations are visible in Figure 7, for both luminances $\mathrm{L}_{\mathrm{L}}$ and $\mathrm{L}_{\mathrm{H}}$. These vectors exhibit a predominating upward direction. The average lens shifts on lowering gaze direction appear to be small: $0.09 \mathrm{~mm}$ with luminance $\mathrm{L}_{\mathrm{L}}$, and $0.23 \mathrm{~mm}$ with luminance $\mathrm{L}_{\mathrm{H}}$.

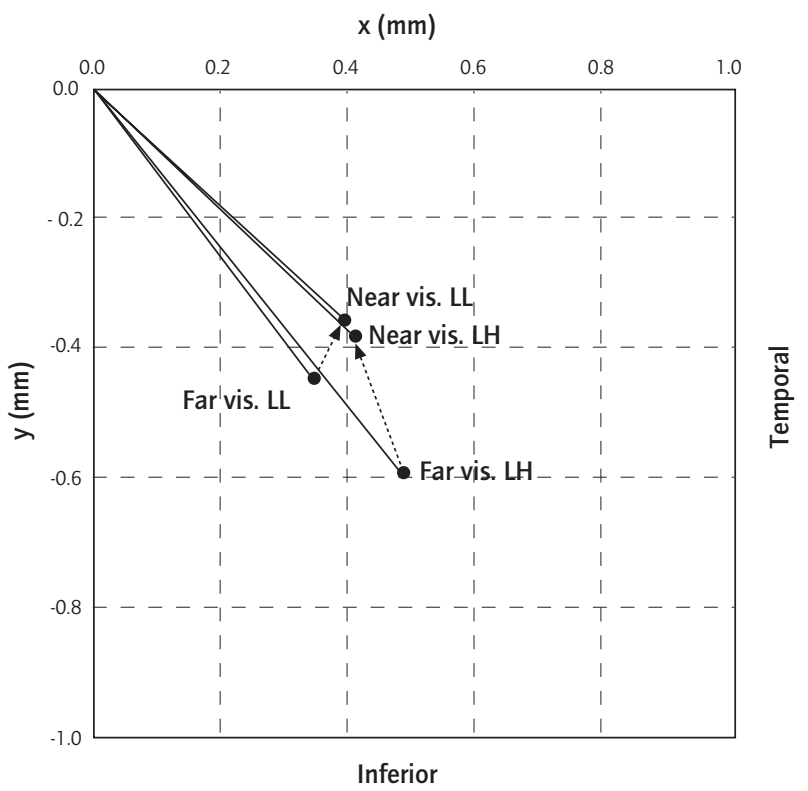

Figure 7. Geometrical representation of mean lens positions in relation to the pupil. The graph origin corresponds to the pupil center; black circles describe mean lens centration in all 4 experimental configurations. Dashed arrows represent mean vector translations between far vision (primary gaze) and near vision (downward gaze). 


\subsubsection{Discussion}

\section{Pupil diameter}

The average pupil size results of Table 2 are larger than those reported by Schäfer and Weale, ${ }^{12}$ probably because their group of presbyopes was older in average than our subjects. We also believe that the repeated use of a flash lamp may have stressed their subjects and influenced their pupil aperture.

Statistical analysis does not reveal differences in pupil diameter between myopes and hyperopes. However, as ametropia is not evenly distributed among our subjects (see Fig. 4), it would certainly be exaggerated to conclude that myopic and hyperopic presbyopes have similar pupil size in average. Despite the limited age range of our subjects, the age-related decrease in pupil size is clearly noticeable in our results (see Fig. 5). However, the slowness of the mean variation of pupil size with age, between 0.02 and $0.04 \mathrm{~mm} /$ year in our experimental conditions, leads us to consider that the mean values of Table 1 are representative of presbyopes between ages of 45 and 60, who are the most likely to wear multifocal contact lenses.

Our results show that the near vision-related contractions of the pupil can not be neglected compared to its diameter changes induced by variations of luminance in the photopic range. This confirms the idea that the optical quality of a refractive multifocal contact lens can benefit from the near pupillary reaction, ${ }^{1,2}$ if the near and far corrections lie respectively in the center and in the periphery of the lens. With such a design, the proximity-induced variations may act in real-time to partially select the corrective zone which is better adapted to the current viewing distance. This mechanism results in an improved contrast of the retinal image, which might explain previous clinical reports ${ }^{21}$ of better subjective acceptance with center addition bifocal contact lenses, in comparison with peripheral addition lenses.

\section{Lens centration}

We were not expecting such a strong tendency in lens decentration (see Fig. 6), since the lenses had generally proven correctly centered on biomicroscopic observation. Decentration mainly occurs in the inferior temporal part of the pupil, as reported by Erickson and Robboy, ${ }^{13}$ who attributed it to combined effects of slight opposite decentrations of pupil and contact lens in relation to the visible iris. As far as we can compare with their rough results, typical length of decentration appears to be smaller with our lenses, probably because of different lens material and geometry.

In far vision, the apparent decrease in average temporal decentration of the lens with lower luminance (Table 3) might be explained by an average shift of the pupil center oriented temporally on dilation, as previously described. ${ }^{15,16}$ It is nevertheless surprising to us that this phenomenon has more influence on the relative position between lens and pupil than the difference in convergence and gaze angle between far and near vision conditions. To keep a proper view of these effects, one must realize that all differences between mean horizontal decentrations of Table 3 are of comparable magnitude with our measuring precision.

In order to explain the apparent effect of luminance on vertical decentration (Table 4), we might consider that a vertical component of the pupil shift on dilation, directed downward 
in average. To our knowledge, such a component was not detected in earlier studies. It is also likely that variations in palpebral aperture induced by luminance changes may affect the contact lens position. In Table 4, the apparent effect of target distance on vertical decentration was more predictable: the slight improvement of centration in near vision is probably due to the downward gaze direction required by the near target. With this posture, the inferior lid may exert more pressure on the lens and reduces its inferior decentration.

The apparent absence of influence of BCR revealed by our analysis requires a careful interpretation. As BCR was adapted to each subject, we did not compare the effects of different $B C R$ values in equivalent subject samples; it would therefore be erroneous to conclude that BCR has no effect on lens decentration. Our results simply suggest that, when soft contact lenses are properly fitted by adjusting their BCR, their position relative to the pupil depends more strongly on external conditions than on individual BCR values.

Our results suggest that ring-marked contact lenses may provide a convenient geometric reference for the measurement of dynamic pupil shifts with an infrared pupillometer. For this particular purpose, it would be preferable to fit the lenses in a rather tight way, in order to minimize their mobility.

The knowledge of systematic decentration of soft contact lenses in relation to the pupil can be turned into an advantage for the optical conception of multifocal contact lenses. For instance, our results allow to compare the tolerance of different optical designs against realistic values of decentration. One could also consider to optimize the lens optical profiles in order to achieve the best possible performance with decentrations corresponding to our average values. Although we think that most soft contact lenses will tend to be shifted in a similar way with respect to the pupil, the strictly quantitative validity of our decentration results is limited to the lens geometries and material (water content) used in our experiments. For instance, our data would not allow to predict the average position of a thinner lens without further experiments. However, similar average decentration is expected with multifocal lenses made in the same material as the tested lenses, because of the very small difference between the surfaces of monofocal and multifocal lenses of same distance powers.

\section{Lens translation}

The topic of lens translation between primary and downward gaze was treated to a great extent in the preceding lines, through the comparison of lens decentrations in far and near vision. One must be aware that the difference between decentrations relative to the pupil yields values of lens translation which are also relative to the pupil. In other words, the translation vectors which we derived represent the vector differences between lens and pupil translations. Despite the fair mobility generally observed when fitting the lenses, the average upward translations on lowering gaze direction remain inferior to $0.25 \mathrm{~mm}$. Our results show that the influence of miosis or mydriasis-related pupil shifts on the relative position between lens and pupil centers can be stronger than that of lens on-eye translations. These new data confirm earlier predictions ${ }^{18}$ of small lens translations on downgaze. They suggest that alternated vision can not be practically achieved with rotationally symmetric soft contact lenses like those used in the present study, and that the principle of so-called simultaneous vision is better suited to these lenses. 
Pupil size and lens centration relative to the pupil only represent a small subset of the factors which are likely to influence visual performance with multifocal contact lenses. ${ }^{1}$ The preceding results may nevertheless help to adapt existing eye models ${ }^{22}$ in order to achieve a more realistic optical simulation of presbyopic multifocal contact lens performance. Theoretical simulation could be further improved by accounting for other clinical data, such as measurements of the Stiles Crawford effect. ${ }^{23,24}$ Both computational and experimental works are now in progress to validate the interest of integrating these physiological data in the optical modeling and design of multifocal lenses.

\subsubsection{Conclusion}

Our videopupillometer measurements in presbyopes fitted with soft ring-marked contact lenses provide us with typical values of pupil size, lens centration and translation in relation to the pupil, under different conditions of luminance, viewing distance and gaze angle. The results also describe several interesting effects: they quantify the slow decrease rate of pupil diameter with aging within the presbyopic range; they reveal that the near vision-related miosis and the pupil contraction due to increased luminance within the photopic range are comparable in magnitude; our data confirm that soft contact lenses are generally shifted in the inferior temporal quadrant of the pupil, and show that both gaze angle and luminance have small yet statistically significant effects on the relative position between lens and pupil centers.

\section{Acknowledgements}

Thanks are due to D. Baude and G. Obrecht for several helpful discussions, to O. Chollot and C. Grollier for their support in experimental work, and to V. Dentan for reviewing the script. Three authors, NC, FB and HM, are employees of Ocular Sciences.

\section{References}

1. Charman, W.N. and Saunders, B. Theoretical and practical factors influencing the optical performance of contact lenses for the presbyope. J Br Contact Lens Assoc 1990;13:67-75.

2. Baude, D. and Miège, C. Presbyopia compensation with contact lenses - a new aspheric progressive lens. J Br Contact Lens Assoc 1992;15:7-15.

3. Baude, D. Caractérisation, optimisation et réalisation de composants optiques multifocaux diffractifs et réfractifs. PhD thesis, Université de Paris-Sud, France, 1990.

4. Borish, I.M. Pupil dependency of bifocal contact lenses. Am J Optom Physiol Opt 1988;65:417-423.

5. Erickson, P., Robboy, M., Apollonio, B.S. and Jones, W.F. Optical design considerations for contact lens bifocals. J Am Optom Assoc 1988:59:198-202.

6. Birren, J.E., Casperson, R.C. and Botwinick, J. Age changes in pupil size. J Gerontol 1950;5:216-224.

7. Kadlecova, V., Peleska, M. and Vasko, A. Dependence on age of the diameter of the pupil in the dark. Nature 1958;182:1520-1521. 
8. Loewenfeld, I.E. Pupillary changes related to age. Topics in Neuro-ophtalmology, (Ed. Thompson, H.S),p 124-150. Williams and Wilkins, Baltimore, USA, 1979.

9. Thompson, H.S. The pupil. Adler's Physiology of the eye, 7th edition, (Ed. Moses, R.A.), p 326-356. C.V.Mosby publisher, St Louis, MO., USA, 1981.

10. Lowenstein, O. and Loewenfeld, I.E. The pupil. The Eye, 2nd edition, (Ed. Davson, H.), vol. 3, p 255327. Academic Press, New York, USA, 1969.

11. De Groot, S.G. and Gebhard, J.W. Pupil size as determined by adapting luminance. J Opt Soc Am 1952;42:492-495.

12. Schäfer, R. and Weale, R. The influence of age and retinal illumination on the pupillary near reflex. Vision Res 1970;10:179-191.

13. Erickson, P. and Robboy, M. Performance characteristics of a hydrophilic concentric bifocal contact lens. Am J Optom Physiol Opt 1985;62:702-708.

14. Woods, R.L., Saunders, J.E. and Port, M.J. Concentric-design rigid bifocal lenses, part II: visual performance. J Br Contact Lens Assoc 1993;16:25-36.

15. Walsh, G. The effect of mydriasis on the pupillary centration of the human eye. Ophtal Physiol Opt $1988 ; 8 ; 178-182$.

16. Wilson, M.A., Campbell, M.C.W. and Simonet, P. Change of pupil centration with change of illumination and pupil size. Optom Vis Sci 1991;2:129-136.

17. Mandell, R.B. Contact lens practice, 4th edition, (Ed. Mandell, R.B.), p 785-823. C.C. Thomas publisher, Springfield, IL., USA, 1988.

18. Borish, I.M. and Perrigin, D. Relative movement of lower lid and line of sight from distant to near fixation. Am J Optom Physiol Opt 1987;64:881-887.

19. Guillon, M. Basic contact lens fitting. Contact lens practice, (Ed. Ruben, M., and Guillon, M.), p 587-622. Chapman \& Hall Medical, London, UK, 1994.

20. CIE Technical Committee 3.1 An analytic model for describing the influence of lighting parameters upon visual performance vol. II : summary and application guidelines, Publication CIE nº19/2.2, p 6064. Bureau Central de la CIE, Paris, France, 1981.

21. Back, A.P., Holden, B.A. and Hine, N.A. Correction of presbyopia with contact lenses : comparative success rates with three systems. Optom Vis Sci 1989;66:518-525.

22. Smith, G. Schematic eyes : history, description and applications. Clin Exp Optom 1995;78:176-189.

23. Stiles, W.S. and Crawford, B.H. The luminous efficiency of rays entering the eye pupil at different points. Proceedings of the Royal Society (London) B, 112, p 428-450, 1933.

24. Applegate, R.A. and Lakshminarayanan, V. Normal variation in the Stiles Crawford effect peak location and rho values. OSA Technical Digest series, 3, 80-83, 1993. 


\subsection{Tear Film Fluorescence}

Testing Fluorescein Agents as a Diagnostic Tool and a Coating for Anterior Eye Topography: A Feasibility Study

J. de Brabander, F.H.M. Jongsma, J.I.J.H. van Rooij and F. Hendrikse.

From:

Eye research Institute Maastricht, Department of Ophthalmology, Academic Hospital University Maastricht, The Netherlands.

Submitted. 
Abstract

Purpose. The purpose of this study was to investigate the effects of excitation wavelength, type of fluorescein, fluorescein concentration, type of dilution fluid and time wash-out effects on transparency/opacity of the tear film in vivo.

Methods. Photographic evidence was used to analyse the visibility of eye tissues under different conditions. Topographic pictures using the Maastricht Shape Topographer (MST) were used to show effectiveness in height measurements in relation to applicated fluorescein concentration and time wash-out effects.

Results. An excitation wavelength of $489 \mathrm{~nm}$ is most effective in obtaining high fluorescent yield. The use of an excitation wavelength of $450 \mathrm{~nm}$, because of its reduced absorption in fluorescein, made deeper structures in the scleral part of the eye visible. Low molecular weight fluorescein was superior in creating opacity compared to high molecular weight fluorescein. Using methyl cellulose as a dilution fluid, increased yield and opacity of the in vivo tear layer. The use of a $4 \mathrm{mg} / \mathrm{ml}$ concentration of applicated fluorescein showed to reveal superior topographic pictures compared to a $2 \mathrm{mg} / \mathrm{ml}$ concentration. Time wash-out effects of the fluorescein are influencing the quality of topographic pictures with the MST device. Stable and high quality pictures could be obtained between 30 and 120 seconds after application of fluorescein.

Conclusion. Fluorescein imaging of the front surface of the eye can be enhanced by an appropriate selection of fluorescein type, concentration and substrate. Using an alternatively excitation wave length of $450 \mathrm{~nm}$ is a way to clinically show the deeper structures in the conjunctival/scleral part of the eye. Small molecular fluorescein is superior in showing details for clinical analysis compared to large molecular fluorescein. The use of higher concentration fluorescein can enhance topographic data of the eye front surface using the MST device. The ideal time to obtain a picture for topographic analysis with the MST device is around 60 seconds after application of fluorescein. Methyl cellulose decreases the time washout effect of fluorescein.

\section{Key words:}

Fluorescein, clinical diagnosis, eye topography, tear film dynamics. 


\subsubsection{Introduction}

Since 1882, fluorescein has been used for clinical diagnosis and research in ophthalmology. ${ }^{1-8}$ Its fluorescent characteristics, such as yield, spectral absorption and emission have been extensively investigated. When exposed to light, fluorescein absorbs the blue and violet parts of the spectrum and emits fluorescent light of longer wavelength after about $10^{-9}$ seconds. ${ }^{7}$ With a spectrophotofluorometer the absorption and emission of a solution of sodium fluorescein in a buffer can be measured. ${ }^{7}$ For low fluorescein concentrations, it shows a maximum absorption at $490 \mathrm{~nm}$. When exposed to this wavelength, the highest intensity of fluorescence is at about $530 \mathrm{~nm} .9$

Traditionally the ophthalmologist observes the eye after instilling fluorescein from a unit dose container or a specially prepared strip, in order to look for absorption of the dye in damaged tissue. Research has shown that fluorescein is capable of staining interruptions in corneal epithelium $2,3,5$, and devitalized ${ }^{10}$ or even living ${ }^{11}$ cells. In both animal and human healthy eyes, daily variability and differences between right and left eyes have been observed. ${ }^{12-15}$

Furthermore, variation in intensity and location of the staining for both pathological and normal eyes has led to the development and use of grid type of grading systems (use of coloured reference images to determine nature and progress of a process). ${ }^{15-18}$

In contact lens fitting, fluorescein is used to estimate the thickness of the tear layer between the posterior lens surface and the corneal epithelium (Fig. 1, c-f). In this case transparency must be sufficient for the excitation light to penetrate the tear layer on the anterior lens surface and the lens itself.

For assessment of tear quality, Tear film Break Up Time (TBUT) and wettability of the eye (Fig. 1, a, b) or contact lens surface (Fig. 1, e-g), one would like the tear film to be visible easily contrasted from the underlying "dry" substance. This requires limited optical penetration (opacity) rather than transparency.

In 1965 Friedberg and Dimond suggested the use of fluorescein as a diffusing coating for corneal topography. ${ }^{19}$ In this application the dye must perform two tasks. One task is to destroy the directional information of the excitation light by transforming the impinging light into a (diffusely) fluorescing image of sufficient luminance. The other task is to make the transparent precorneal tear film opaque for the excitation light. When the tear film is insufficiently opaque to the excitation wavelength, backscatter from underlying eye tissues can disturb the fringe pattern, especially from the posterior corneal surface and iris (e.g. from the iris in blue-eyed individuals). Sometimes, also the bulbar conjunctiva that covers the white scleral tissue must be mapped, implying that requirements concerning the opacity are high for all types of eyes. This suggests the use of fluorescein solutions of high concentrations (high absorption of excitation light) with consequently a reduced fluorescent yield due to concentration quenching. Fortunately, fluorescein is a fluorescent agent with a high quantum yield. ${ }^{20}$ Nevertheless, it would be helpful to know the behaviour of the fluorescent label under controlled circumstances for example layer thickness and fluorescein concentration.

The aim of the present study was to explore the feasibility of quantifying the efficiency of fluorescein applications using various types of fluorescein, concentrations and dilution fluids. 

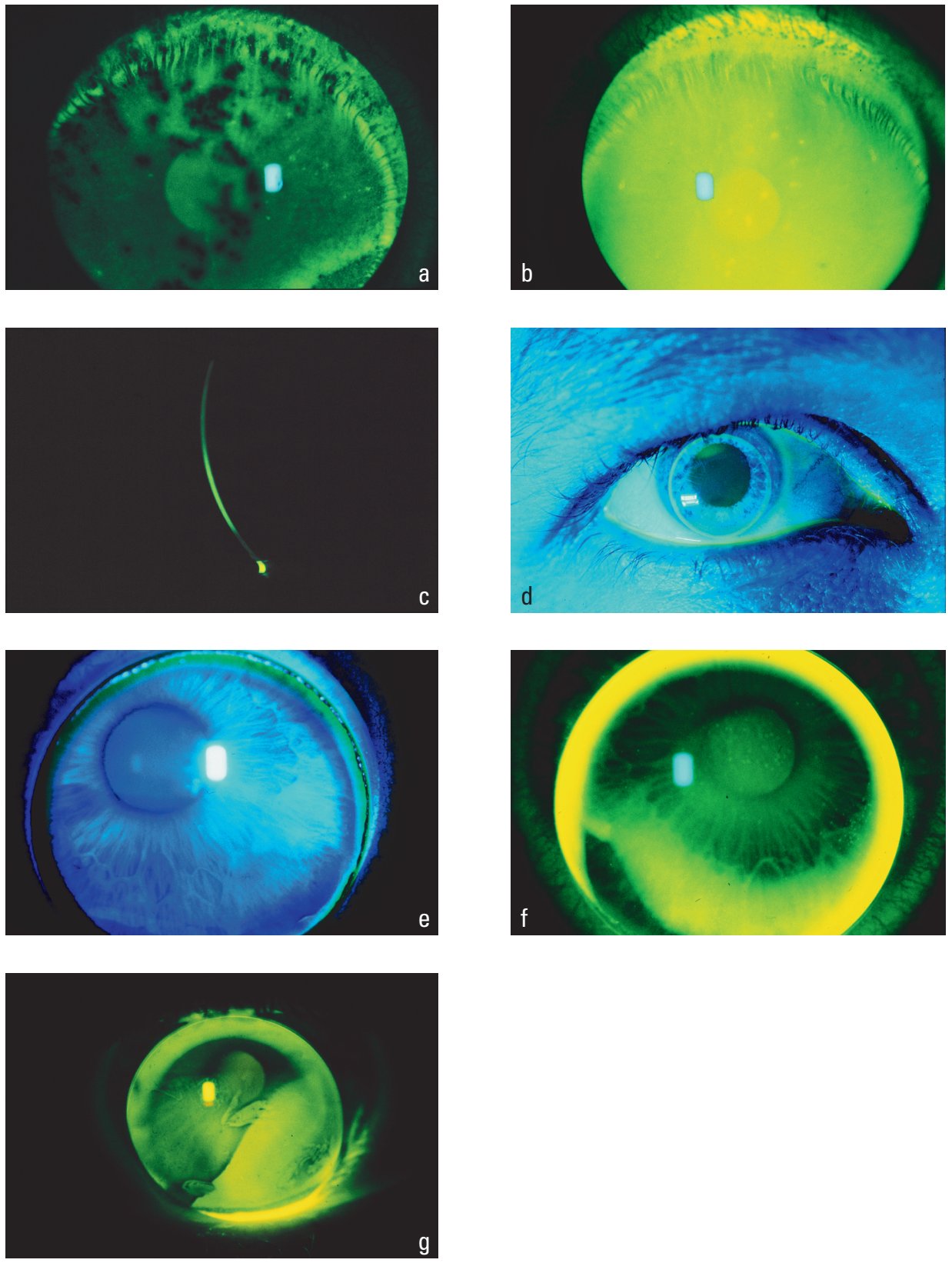

Figure 1. Various applications of fluorescein in contact lens fitting and assessment of tear quality and wetting (see text introduction). 


\subsubsection{Materials and methods}

Photographs of a blue eyed volunteer's eye were taken using a Zeiss 75SL photo slitlamp. Within the illumination path of the slitlamp, excitation filters with a centre wavelength of 450 and $489 \mathrm{~nm}$ and a $10 \mathrm{~nm}$ Full Width Half Maximum bandwidth were placed. Large and small molecular fluorescein was used from sterile single-use strips. Saline, and methyl cellulose $0.5 \%$ and $2 \%$ served as dilution fluids. The photographs were taken on a 200 ASA colour positive film, 60 seconds after instilling one drop of the fluorescein solution under test. Minimum time between tests was 4 hours. The photographic material was visually analysed on visibility of the underlying tissue (transparency/opacity) and on intensity of colour (fluorescence).

Topographic pictures taken with the Maastricht Shape Topographer (MST) ${ }^{21}$ were analysed on quality using prepared fluorescein concentrations of $2 \mathrm{mg} / \mathrm{ml}$ and $4 \mathrm{mg} / \mathrm{ml}$. In order to avoid mixture of the different concentrations the testing was done on two separate days, but on all occasions 60 seconds after application of the fluorescein. In an other experiment the same eye was repeatedly measured at 30, 90, 150 and 240 seconds after the application of fluorescein.

\subsubsection{Results}

Figure 2 (top a, b) shows a pair of photographs of the same eye after instilling small molecular sodium fluorescein diluted in saline. The left picture was taken with $450 \mathrm{~nm}$ excitation wavelength and the right picture with $489 \mathrm{~nm}$ excitation wavelength. As the chromatic extinction coefficient for $450 \mathrm{~nm}$ is less than for $489 \mathrm{~nm}$, the deeper structures at the scleral part of the eye are more clearly visible with the shorter wavelength.

In Fig. 2 (bottom c, d) the difference is shown between the photographic result after instilling small (c) and large (d) molecular fluorescein diluted in saline. The small molecular fluorscein shows a higher opacity than the large molecular.

Figure 3 shows four pictures where methyl cellulose instead of saline was used as solvent for fluorescein. For both the methyl cellulose $2 \%$ (b) and $0.5 \%$ (d), the small molecular fluorescein (a, c) outperforms the large molecular (b, d) in both yield and opacity.

The images as obtained with the MST and fluorescein concentrations of $2 \mathrm{mg} / \mathrm{ml}$ and 4 $\mathrm{mg} / \mathrm{ml}$ are shown in Fig. 4. The used substrate was methyl cellulose $0.5 \%$ and the images were taken 30 seconds after application. The use of the lower concentration (left two pictures) revealed a fluorescein yield under the threshold for a reliable analysis of topography. This can already be deducted from the left/top picture showing the raw image of one of the grids projected on the eye by the MST device. The left/bottom picture clearly shows that the outcome of the topographic analysis using the lower concentration of fluorescein is unreliable. The right two pictures of Fig. 4 show the projected grid (top) and the outcome of the topographic analysis (bottom) for the higher concentration fluorescein. A reliable topography of the entire front surface of the eye, including the tear prism at the lower eye lid could be obtained. 


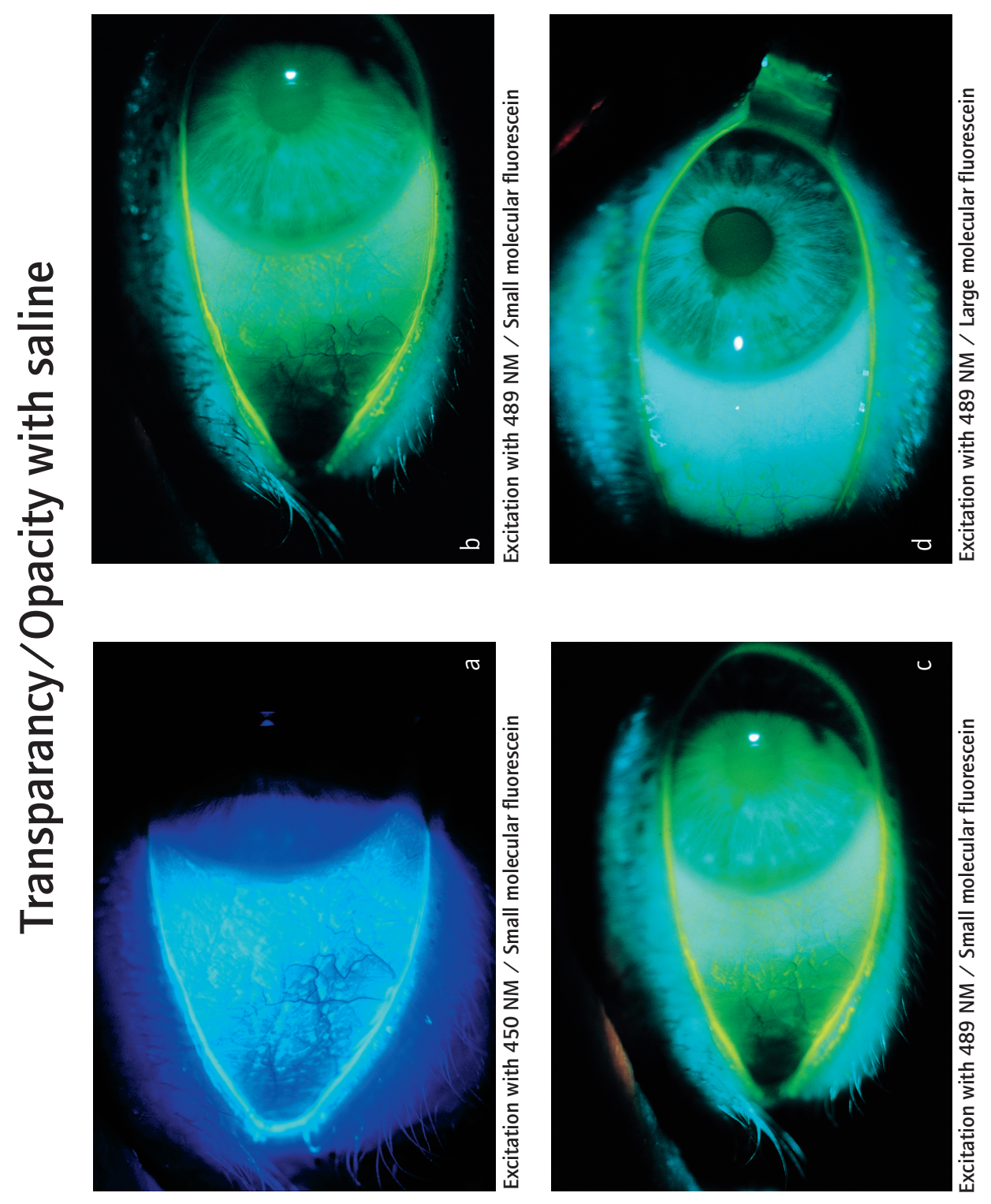

Figure 2. Transparency /opacity with saline. The top pictures show the difference in opacity between the excitation with $450 \mathrm{~nm}$ (a) and $489 \mathrm{~nm}$ (b) using a small molecular fluorescent dye. Note the better visibility of the underlying episcleral tissue in a. The bottom pictures show the difference in opacity using small (c) and large (d) molecular fluorescein with saline, and an $489 \mathrm{~nm}$ excitation wavelength. 


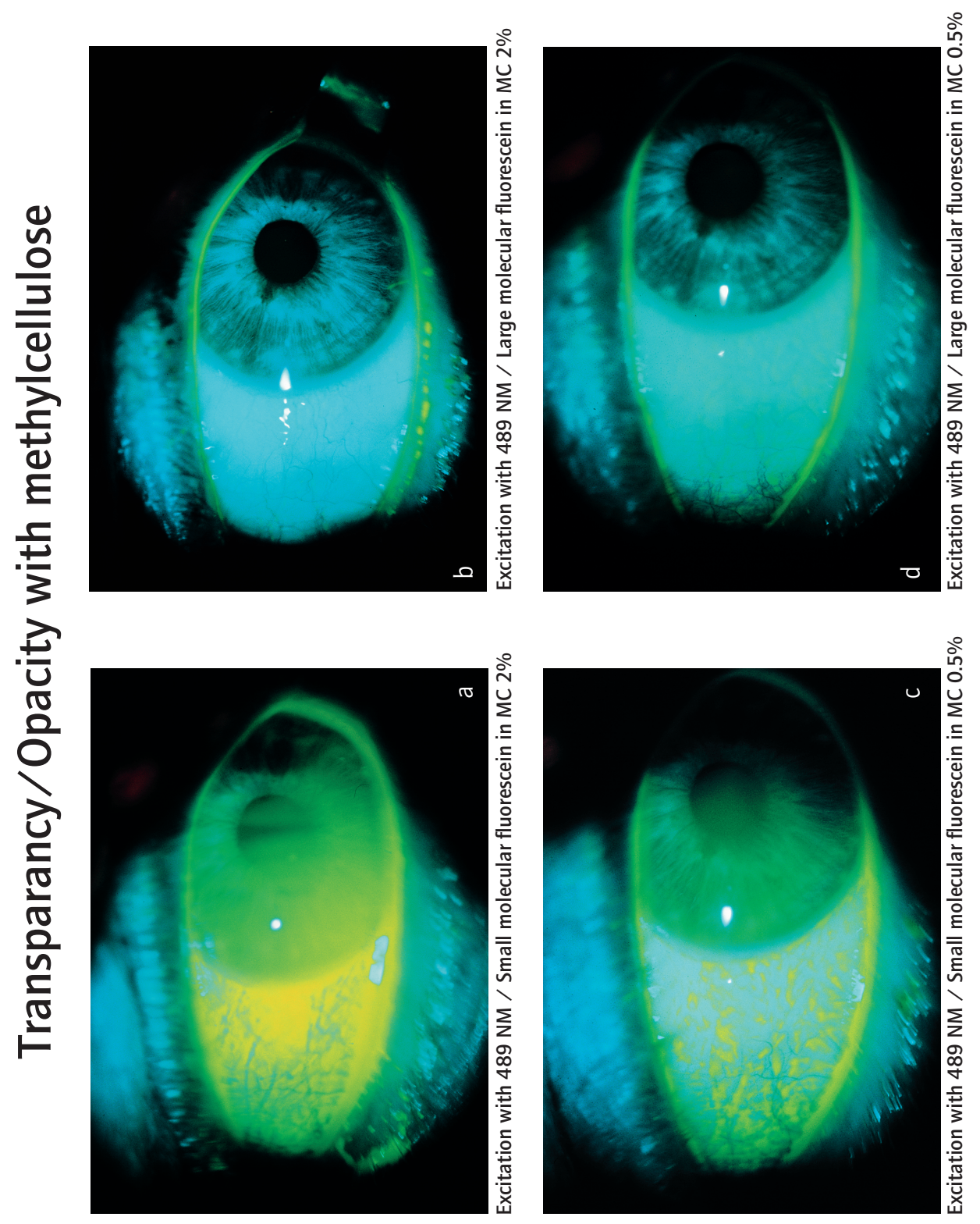

Figure 3. Four photographes of a the same eye using $489 \mathrm{~nm}$ excitation wavelength. The left pictures were taken using small molecular fluoresceine, for the right pictures large molecular fluoresceine was used. In the upper pictures methylcellulose $2 \%$ served as substrate, in the lower pictures the substrate was methylcellulose $0.5 \%$. Note the remarkable higher opacity with small molecular fluorescein and the higher concentraton methylcellulose in the upper/left picture. 
The time wash-out effects of fluorescein using methyl cellulose $0.5 \%$ and $\mathrm{NaCl} 0.9 \%$ as substrate are shown in Fig. 5. The top series of pictures show the projected grid on the eye at 30, 90, 150 and 240 seconds after the application of fluorescein with methyl cellulose $0.5 \%$. The middle series of picture shows the analysis of topographic data using the raw images as shown at the top. At the bottom, the topographic data is presented for the use of $\mathrm{NaCl} 0.9 \%$ as a substrate for the fluorescein. The degradation of the images due to time wash-out effects is clearly shown. Also, it can be deducted from Fig. 5 that with the use of methyl cellulose $0.5 \%$ as a substrate, the influence of time wash-out effects is lower than with $\mathrm{NaCl} 0.9 \%$. Reliable reconstruction of the eye surface was possible up to $150 \mathrm{sec}$. and 90 sec. for methyl cellulose $0.5 \%$ and $\mathrm{NaCL} 0.9 \%$, respectively.
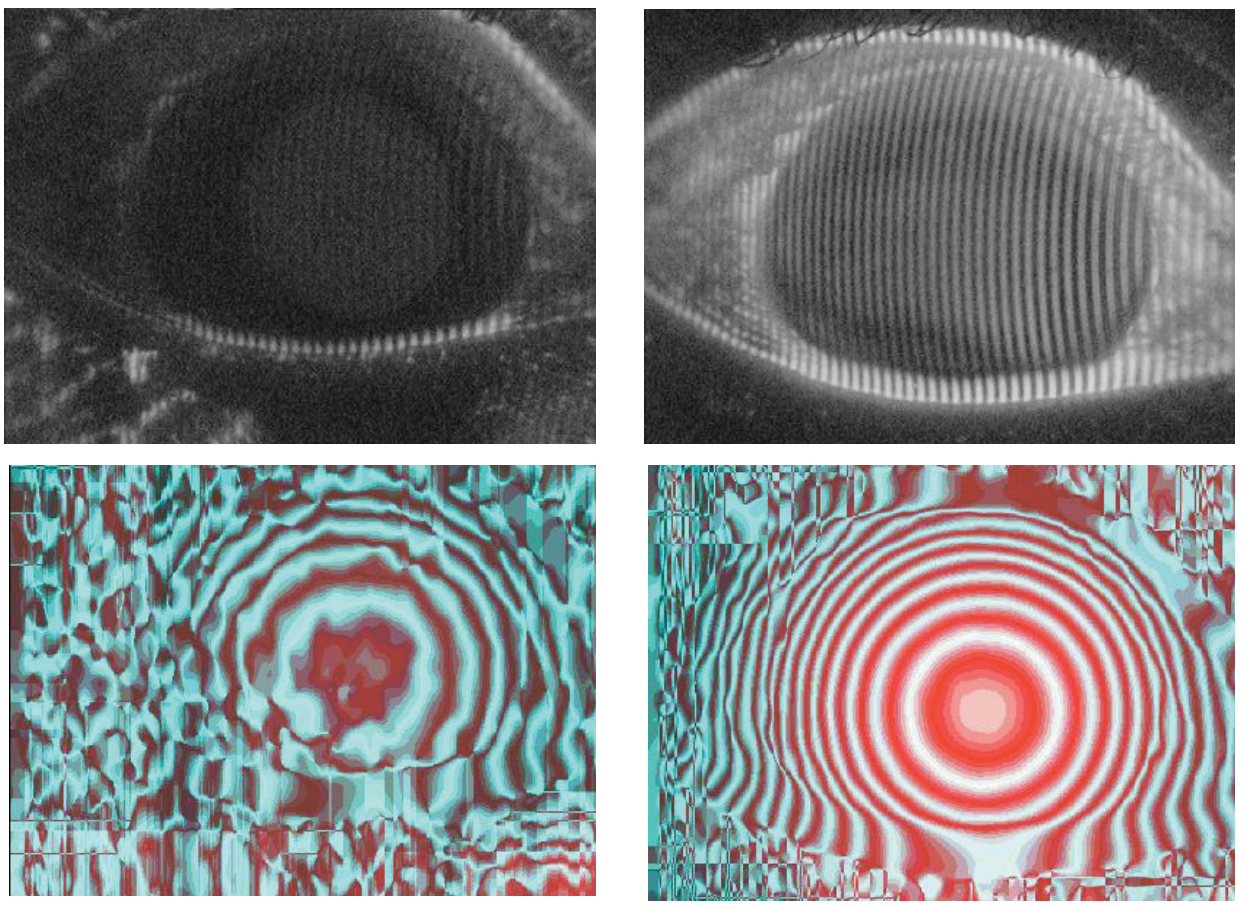

Figure 4. Topographic imaging of the eye front surface using fluorescein concentrations of $2 \mathrm{mg} / \mathrm{ml}$ (left) and 4 $\mathrm{mg} / \mathrm{ml}$ (right). The used substrate was methyl cellulose with imaging 30 seconds after application. Shown are, the raw images of one of the projected grids on the eye (top), and the analysis of height topography (bottom).

\subsubsection{Discussion}

Better visibility of the deeper structures in the scleral part of the eye at shorter wavelength excitation (Fig. 2 a) can be explained by the lower light absorbance for $450 \mathrm{~nm}$ than for 489 $\mathrm{nm}$. Probably, the same result can be obtained using longer wavelengths at a more diluted solution. In clinical practice it is rather difficult to exactly control the concentration of the fluorescein in the eye. One could, by rinsing the strip before utilization, reach a lower con- 

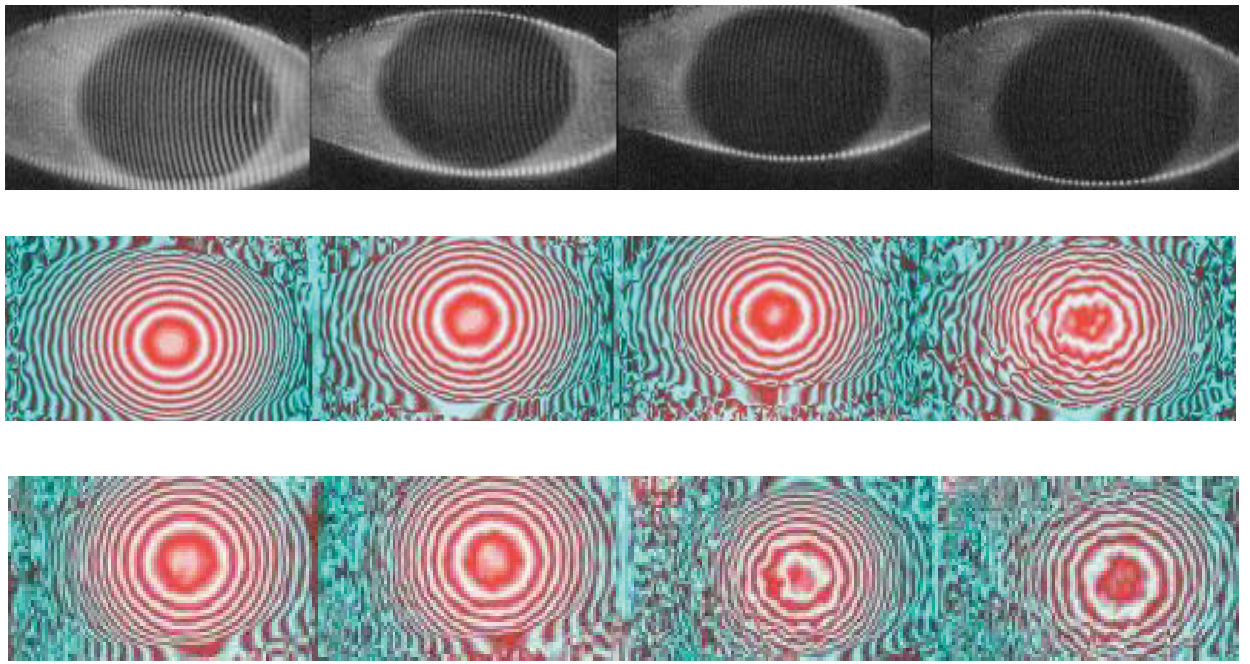

Figure 5. Topographic imaging of the eye front surface at 30,90, 150 and 240 seconds after the application of 4 $\mathrm{mg} / \mathrm{ml}$ fluorescein in the substrate. The two series at the top show the projected grid on the eye and the results of the topographic analysis with methyl cellulose $0.5 \%$ as a substrate for the fluorescein. The bottom series presents the topographic analysis with $\mathrm{NaCl} 0.9 \%$ as a substrate.

centration or wait until the tears dilute the fluorescein. A practical solution allowing observations at wavelengths of not only $489 \mathrm{~nm}$ but also $450 \mathrm{~nm}$ would be to incorporate a 450 $\mathrm{nm}$ filter into the slit lamp filter disk. It would be of interest to investigate this aspect further since no clinical assessment of the scleral/conjunctival structures with a viewing system at 450 nm wavelength has been performed.

From Fig. 3 it is clear that small molecular fluorescein results in better opacity. This is in accordance with our general observations in performing height topography with the MST. ${ }^{21}$

The addition of methyl cellulose increases fluorescence yield and also decreases time wash-out effects (Fig 5). Theoretically this would be an advantage in topographic measurements. However, it is unclear whether this increase is due to a higher fluorescence efficiency or to an increase of layer thickness caused by the viscosity of the methyl cellulose. With the addition of methyl cellulose, the increase in the thickness of the tear film could possibly obscure distinct local height differences. The balance between detection of small height differences vs. the better possibility of obtaining height measurements over the entire eye needs further study.

In summary, this feasibility study shows that with an appropriate selection of fluorescein type, concentration and substrate it is possible to enhance fluorescein imaging for planned clinical purposes. For routine topographic imaging with the MST device, it seems that optimal results can be achieved with the use of a $4 \mathrm{ml} / \mathrm{mg}$ fluorescein concentration and imaging around 60 seconds after application. The choice between $\mathrm{NaCl} 0.9 \%$ or methyl cellulose $0.5 \%$ as a substrate for the fluorescein offers a new way to, respectively obtain more detailed topographic data (e.g. in corneal irregularities) or shape measurements of the entire front surface of the eye (e.g. in scleral contact lens fitting). 


\section{References}

1. Maurice DM. The use of fluorescein in ophthalmological research. Invest Ophthalmol 1967;6;5:464477.

2. Fromm, Groenouw. Ueber die diagnostische Verwendbarkeit der fluoresceinfarbung bei Augenerkrankungen. Arch Augenheilkd 1891;22:247-57.

3. Reitsch. Farbung der oberflaechliche erkrankten Hornhaut mit Fluorescein und biebrichler Scharlachrot. Graefes Arch Opthalmol 1915;89:299-307.

4. Passmore JW, King JH. Vital staining of conjunctiva and cornea. Arch Ophthalmol 1955;55:568-574.

5. Norn MS. Vital staining of cornea cornea and conjunctiva. Acta Opthalmol (Kbh) 1962;40:389-401.

6. Baily EA, Rollefson GK. The determination of the fluorescent lifetimes of dissolved substance by a phase shift method. J Chem Phys 1953;21:1315-1322.

7. Pflueger. Zur Ernaehrung der Cornea. Klin Monatsbl Augenheilkunde 1882;20:69-81.

8. Romanchuk KG. Fluorescein. Physicochemical factors affecting its fluorescence. Surv of Ophthalmol 1982;26;5:269-283.

9. Delori F, Ben-Sira I, Trempe C. Fluorescein angiography with an optimized filter combination. Am J Ophthalmol 1976;82;4:559-566.

10. Tabery HM. Dual appearance of fluorescein staining in vivo of diseased human corneal epithelium. A non-contact photomicrographic study. Br J Opthalmol 1992;76:43-4.

11. Feenstra RPG, Tseng SCG. Comparison of fluorescein and rose bangal staining. Ophthalmology 1992;99:605-17.

12. Kikkawa Y. Normal corneal staining with fluorescein. Exp Eye Res 1972;14:13-20.

13. Caffery BE, Josephson JE. Corneal staining after sequential installation of fluorescein over 30 days. Optom Vis Sci 1991;68:467-9.

14. Schwallie JD, McKenney CD, Long WD Jr, McNeil A. Corneal staining patterns in normal non-contact lens wearers. Optom Vis Sci 1997;74:92-8.

15. Josephson JE, Caffery BE. Corneal staining characteristics after sequential instillation of fluorescein. Optom Vis Sci 1992;69:570-73.

16. Korb DR, Herman JP. Corneal staining subsequent to sequential fluorescein instillations. J Am Optom Assoc 1979;50:361-7.

17. Josephson JE, Caffery BE. Corneal staining after instillation of topical anesthetic (SSII). Invest Ophthalmol Vis Sci 1988;29:1096-9.

18. Schwallie JD, Long WD Jr, McKenny CD. Day to day variations in ocular surface staining of the bulbar conjunctiva. Optom Vis Sci 1998;75:55-61.

19. Friedberg MA and Dimond WJ. Method for determining surface contours of an eye. US patent No. 3,169,459, 1965.

20. Pringsheim P, Vogel M: Luminicence of liquids and solids and its practical applications. Interscience, New York, 1943.

21. Jongsma FHM, de Brabander J, Hendrikse F, and Stultiens BAT. Development of a wide field height eye topographer: Validation on models of the anterior eye surface. Optom Vis Sci 1998;75:69-77. 
Chapter 6

Summary and Conclusions 


\section{Summary and Conclusions}

Contact lenses are widely used to correct refractive error of the eye, to serve as a bandage in eye pathology, to provide a prosthetic aid in deformations of the iris or front segment of the eye, and to act as a cosmetic device to change the colour of the iris. The success and safety of wearing contact lenses relate to a complex of factors, of which the following are the main ones: the basic condition of the eye, the design and the material of the contact lens, the physiology of the front segment of the eye with the lens in situ, comfort of wear, visual correction, the efficacy of the care system, and the compliance of the wearer. Prescribing contact lenses is traditionally based on trial-and-error fitting, combined with evaluation and modification of the lens. This needs experienced practitioners; it is time consuming, and difficult to reproduce. Computer-aided manufacturing, however, makes it possible to produce contact lenses with precision and reproducibility on a sub-micron level. This technique allows practitioners not only to fit the lens more precisely on the eye, but also to improve its optics. Yet, to fully explore these techniques, detailed data on the topography of the front segment of the eye and the optical properties of the eye are essential. Recent developments in contact lens materials make it possible theoretically to minimise the influence of the contact lens on the physiology of the eye. The question is whether these favourable properties can be assured over longer time periods of wear. The efficacy of care systems that are specially developed for these materials, play, together with patient compliance, an important role in final success.

This thesis describes several studies concerning the clinical application of recently developed materials and production techniques in relation to contact lens designs, fitting methods and care systems. The thesis reports on the medical application of contact lenses (chapter 2), the design and care of rigid high gas permeable contact lenses (chapter 3), optical advancements in custom-made soft contact lenses (chapter 4), the development and testing of new methods to measure the front surface of the eye and the pupil, and methods to evaluate the tissues of the front surface of the eye using fluorescein (chapter 5).

\section{Chapter 2}

High oxygen permeability can be obtained with lenses made of silicone. We have fitted these, Silsoft contact lenses, in children with aphakic eyes after surgery for congenital cataract (2.2). The fitting procedure as used in this study proved that without keratometry one can prescribe silicone lenses in very young aphakic patients after congenital cataract in an easy and logical way. An initial TD of $11.3 \mathrm{~mm}$ and BOZR of $7.50 \mathrm{~mm}$ are recommended to give a high success rate. This study demonstrated the feasibility of permanent wear of Silsoft contact lenses in young aphakic children up to 3 years of age, with a very low complication rate. However, strict follow-ups, replacement of the lenses whenever needed, an intensive instruction to co-operative parents, and a practice that is open for emergencies on a 24-hour basis, are essential requirements for providing optimal care in this patient group. Mean lens usage of 5.6 lenses per eye per year is necessary to successfully correct aphakic children after congenital cataract with silicone contact lenses. It seems that from the 
age of 3 years silicone lenses become less compatible with tear composition and should in most cases be replaced by daily-wear high-water content soft lenses, or at a later age with HGP lenses. The achieved visual acuity at the end of this study was comparable to reports in the literature. This suggests that successful optical correction in infant aphakia on a permanent wear basis using Silsoft contact lens can be recommended as a first choice option for aphakic children up the age of 3 years.

The application of thin high-water-content lenses as an artificial iris and black occluding pupil has been investigated in 34 eyes for their ability to provide a cosmetic prosthesis (2.3). The lenses used were available in 5 colours and incorporated standard dimensions of iris $(12.5 \mathrm{~mm})$ and pupil $(5.0 \mathrm{~mm})$. In all cases blocking of hindering light could be achieved. In monocular application, $67 \%$ of the patients were satisfied with the cosmetic effect of the lens compared to the other eye. This means that for the majority of patients the troublesome way of fitting hand-painted contact lenses can be avoided. Because of discoloration of the lenses, it is recommended to replace the lenses every six months. This fits within the general practice to replace hydrophilic contact lenses regularly.

\section{Chapter 3}

The availability of high gas permeable (HGP) contact lens materials has been one of the major steps in contact lens technology. Problems with the first generation of these new materials have been the geometric instability and the formation of deposits on their surface, leading practitioners to hesitate to use these types of lenses. A similar situation has developed concerning the application of aspherical lens designs. Technological advances in the production of the contact lens materials, the physical production of rigid gas permeable (RGP) lenses using computer-numeric-controlled (CNC) techniques, and the availability of new fitting and care systems, have led us to investigate the clinical results of modern HGP contact lenses.

In a study involving 68 patients and 1-year follow-up, we compared the success of spherical and aspherical rigid contact lenses with low and high oxygen permeability (3.2). In $77 \%$ of the cases the patients were able to wear the lenses all day, in $11 \%$ the wearing time was limited, and $12 \%$ had stopped wearing the lenses. Limited wear and failure were related to wearing comfort, lens surface drying, and corneal epithelium compromise (3-9 o'clock staining). No significant differences were found between the clinical results and stability of the tested materials. This means that there is no technical restriction to using aspherical rigid contact lenses in high gas permeable material.

The favourable results from the study described above led us to further investigate the methodology and care of aspherical HGP contact lenses (3.3). In this study 66 patients were fitted with aspherical HGP contact lenses. Employing random selection, we used two different fitting procedures and two different care systems. With 1-year follow-up, $85 \%$ of the patients were able to wear the lenses successfully every day, $7.5 \%$ reported limited wear, and $7.5 \%$ refrained from wearing them. Limited wear and failure were related to wearing comfort, lens surface drying, and 3-9 o'clock staining. A newly developed scheme for selection of lens diameter proved to predict the final lens diameter $(90 \%)$ significantly better than the method of using the visible vertical iris under supra-version (72\%). A remarkable result from 
this study was that the success of the topographic method to predict the base curve of the lens $(75 \%)$ was lower than with the method in which solely keratometer readings were used $(83 \%)$. Although the difference was not statistically significant, one would have expected the topographic method to be better. Further analysis of the corneal topography showed that failures in the prediction of the BOZR could be attributed to larger differences in flattening (E-value) between quadrants than with correct predictions. A method based on computation using weighted averages of corneal flattening in four quadrants would theoretically increase the success in predicting the BOZR to $92 \%$. Although the predictive value of this new system is high, it is not high enough to be used as a system for direct ordering without the involvement of a competent contact lens practitioner. After 1 year of use it was shown that the care system including a mechanical cleaner and the all-in-one system were able to keep the lenses in good condition in relation to protein deposits and geometrical stability in $79.6 \%$ and $79.4 \%$ of the cases, respectively. The use of an all-in-one care solution seems the best option to guarantee good daily care, in relation to patient's compliance.

We conclude that HGP contact lenses can be successfully used, given that the lenses are well manufactured, efficiently fitted, and proper cared for. Involving a contact lens practitioner still applies through lens selection, evaluation and follow-up, an important factor in reaching success.

\section{Chapter 4}

Modern CNC lathe techniques facilitate the extension of the application of hydrophilic soft contact lenses for optical correction of astigmatism, presbyopia, spherical aberration in high ametropia, and severe optical aberration as in keratoconus.

The study on custom-made toric soft contact lenses showed that a relative easy system of ordering from eye parameters can be used successfully (4.2). This study involved 193 eyes with astigmatism between 1 and 7.5 D. Toric soft contact lenses were manufactured based on spectacle refraction, corneal radii, and horizontal visible iris diameter. The percentage of success was $69 \%$ for new wearers, and $54 \%$ for patients that had been failures regarding wearing other toric soft lenses. In cases where the initial lens was not successful, the ordering of a second or third lens could increase the percentage of success to $92 \%$. For former failures of other toric soft lenses this percentage was significantly lower (74\%). With these results, it seems that the troublesome fitting via trial lenses can be avoided. However, from our results it is also clear that this system in its present state is insufficiently robust to be used as a direct ordering system without the involvement of a contact lens practitioner.

In studying the application of contact lenses to correct presbyopia, we could, through an intensive literature study, contribute to the detailed description of the complexity of this, increasingly popular, lens modality (4.3). In a clinical study involving 90 patients, the selection from 5 different prototype varifocal contact lenses showed a success rate of $67.8 \%$. Success in distance vision was entirely related to the optical profile of the lenses; near vision success was related to a combination of addition and pupil size. It is important to conclude that with the specially designed lenses we have used, we were able to increase the success rate in correcting the presbyopic patient in general, but that optimal visual functioning can be 
further enhanced if the lens profiles could be individually designed.

Our results on testing specially designed aspherical lenses showed, in a study with 122 high ametropic eyes, that a systematic compensation of spherical aberration of the contact lens is of no use on average (4.4). However, in approximately $30 \%$ of the cases, contrast sensitivity function was found to be improved. This means that, if we were able to measure the (spherical) aberration of the individual eye, we would, with today's technology, also be able to correct it.

The theoretical possibilities of correcting extreme optical aberrations have been investigated using corneal topographic data of 9 eyes with severe keratoconus (4.5). Depending on the amount of optical aberration, perfectly centred lenses could improve the contrast sensitivity function 6.5 to 200 fold. However, translation and rotation of the lens can degrade this gain. With rotation of the lens up to 10 degrees, the gain remains a factor of 3 or more. Translation is more critical and should be limited to $0.5 \mathrm{~mm}$ in order to reach an improvement in contrast sensitivity.

In this chapter we have shown that the introduction of newly developed hydrophilic soft contact lenses has substantially increased the possibilities of optical correction. Good centring and minor movement of these lenses offers unique opportunities to correct higher order aberrations. However, gain in visual function can only be reached by measuring the optical properties of the eye, and by individually designing and manufacturing the corrective lens. Moreover, the involvement of a contact lens practitioner is essential in reaching success.

\section{Chapter 5}

As described earlier, the new possibilities to correct the eye by means of contact lenses also demand detailed measurements. In this chapter we reported on the development and testing of newly developed instrumentation and methodology to measure the eye, and to evaluate its front surface.

The evaluation of the Maastricht Shape Topographer (MST) showed that this device is able to unambiguously measure the topography of the entire eye surface (5.2.1). This device, based on Fourier profilometry, is able to present $20 \times 16 \mathrm{~mm}$ of the front surface of the eye as a height map or a cross section with high degree of accuracy.

The study, comparing corneal-scleral profiles as measured with the MST height topographer in normal and keratoconus eyes (5.2.2), showed that in keratoconus the asymmetrical angles of the limbal areas, as found in normal eyes between nasal and temporal, is absent. Meridional cross-sections of the entire eye surface can be a good starting point for designing (semi-) scleral contact lenses.

The study with a specially developed pupillometer (5.3) revealed some important characteristics of the pupil in far and near reading tasks and under different conditions of illumination. This understanding can be of importance to further improve contact lens designs, especially for varifocal contact lenses.

Finally, the results of the study on fluorescein phenomena (5.4) revealed that a well-chosen combination of excitation wavelength and the type, concentration and substrate of fluores- 
cein makes it possible to visualise ocular structures in accordance with clinical wishes, and to enhance the reliability of the height measurements of the front surface of the eye.

In summary, this thesis shows that today a variety of excellent options to correct the eye by means of a contact lens are available. The implementation of new developments in technology can improve the fitting, the physiological response, and the efficient care of contact lenses. Improved techniques to measure the eye, computer-aided lens design and computernumeric-controlled manufacturing make it possible to correct almost all optical defects of the eye including higher order aberrations.

Our studies also show that to fully explore these new developments in contact lens technology, the involvement of a specialist in the contact lens field is an important requirement. The work of the specialist will in the future shift from trial-and-error fitting and modification of the lens to measuring, designing and follow-up. The use of methods developed in this study for objectively measuring the eye, designing the contact lens, and evaluating the fit and physiological response, can be of value in achieving optimal results. 


\section{Samenvatting en conclusies}

Contactlenzen worden op grote schaal toegepast bij refractieafwijkingen van het oog, als bandage bij oogpathologie, als prothese bij deformaties van de iris of het voorste oogsegment, en als cosmetisch instrument om de kleur van de iris te veranderen. Het succes en de veiligheid bij het dragen van contactlenzen hangen samen met een complex van de volgende hoofdfactoren: de basisconditie van het oog, het ontwerp en het materiaal van de lens, de fysiologie van het voorste oogsegment met de lens in situ, het draagcomfort, de visuele correctie, de effectiviteit van het onderhoudssysteem en de compliantie van de drager.

Het aanmeten van contactlenzen is traditioneel gebaseerd op de methode van passen, evalueren en modificeren. Dit vereist een hoge mate van ervaring, is tijdrovend en moeilijk reproduceerbaar. Computer gestuurde fabricagetechnieken maken het mogelijk om contactlenzen te vervaardigen met een precisie en reproduceerbaarheid op submicrometer niveau. Hierdoor ontstaan nieuwe mogelijkheden om de lens nog nauwkeuriger aan te meten en tevens de optische correctie te verbeteren. Echter, om deze nieuwe technische mogelijkheden ten volle te benutten zijn gedetailleerde gegevens over de topografie van het voorste oogsegment en de optische eigenschappen van het oog nodig. Recente ontwikkelingen op het gebied van contactlensmaterialen bieden theoretisch de mogelijkheid om de invloed van de lens op de fysiologie van het oog te minimaliseren. De vraag is of de gunstige eigenschappen van deze materialen op langere termijn aanwezig blijven. De effectiviteit van speciaal voor deze materialen ontwikkelde onderhoudssystemen en de mate waarin de drager van de lenzen zich houdt aan de instructies spelen daarbij een belangrijke rol.

Dit proefschrift beschrijft een aantal studies naar de klinische toepassing van recentelijk ontwikkelde materialen en fabricagetechnieken in relatie tot het ontwerp, de aanmeetmethode en het onderhoud van contactlenzen. Het werk is onderverdeeld in de medische toepassing van contactlenzen (hoofdstuk 2), het design en onderhoud van hoog zuurstofdoorlatende vormstabiele contactlenzen (hoofdstuk 3), de optische mogelijkheden van custom-made zachte hydrofiele contactlenzen (hoofdstuk 4), het ontwikkelen van nieuwe methoden om de vorm van het oogoppervlak en de pupil te meten, alsmede om de voorste oogweefsels met behulp van fluoresceïne te evalueren (hoofdstuk 5).

\section{Hoofdstuk 2}

Een zeer hoge zuurstofpermeabiliteit kan worden verkregen met contactlenzen vervaardigd uit silicone materiaal. We hebben deze Silsoft contactlenzen aangemeten bij kinderen met afakie na operatie voor congenitaal cataract (2.2). Het aanmeetsysteem zoals gebruikt in ons onderzoek toonde aan dat men bij afakie na congenitaal cataract, silicone lenzen kan voorschrijven op een veilige en logische manier zonder gebruik te maken van keratometrie. Om tot een hoge successcore te komen wordt als eerste lens een diameter van $11.3 \mathrm{~mm}$ en een BOZR van $7.50 \mathrm{~mm}$ aanbevolen. Permanent dragen van Silsoft contactlenzen bleek in dit onderzoek met weinig complicaties mogelijk bij afake kinderen tot de leeftijd van 3 jaar. Echter, een strikt controlesysteem, vervanging van de lenzen indien nodig, een intensieve instructie aan goed meewerkende ouders en een voor noodgevallen 24 uur bereikbare prak- 
tijk zijn essentiële voorwaarden om een optimale zorg voor deze patiëntengroep te waarborgen. Een lensverbruik van 5.6 lenzen per oog per jaar is noodzakelijk om kinderen met afakie na congenitaal cataract succesvol te corrigeren. Tevens bleek dat vanaf de leeftijd van 4 jaar silicone contactlenzen niet langer compatibel zijn met de traansamenstelling en vervangen dienen te worden door hoogwaterhoudende daglenzen of op latere leeftijd door HGP lenzen. De behaalde visus aan het einde van de studie bleek vergelijkbaar met de getallen uit de literatuur. Dit geeft aan dat optische correctie op permanente basis met de Silsoft contactlenzen kan worden aanbevolen als een eerste keuze bij afake kinderen tot 4 jaar.

Nieuw ontwikkelde, dunne hoogwaterhoudende contactlenzen met een kunstiris en/of zwarte occluderende pupil werden bij 34 ogen onderzocht op geschiktheid als cosmetische prothese (2.3). De gebruikte lenzen waren beschikbaar in 5 kleuren en hadden standaard afmetingen van iris $(12.5 \mathrm{~mm})$ en pupil $(5.0 \mathrm{~mm})$. In alle gevallen kon een blokkade van het hinderlijk invallend licht gerealiseerd worden. Bij monoculaire toepassing was $67 \%$ van de patiënten tevreden over het cosmetische effect van de lens ten opzichte van het andere oog. Dit betekent dat voor het merendeel van de patiënten de moeizame weg van het aanmeten van handgeschilderde contactlenzen kan worden vermeden. In verband met eventuele ontkleuringen van de lens is het raadzaam de lenzen iedere 6 maanden te vervangen. Dit past in het algemene beleid om zachte hydrofiele lenzen regelmatig te vervangen.

\section{Hoofdstuk 3}

Het beschikbaar komen van hoog zuurstofdoorlaatbare (HGP) contactlensmaterialen was één van de belangrijke stappen in de contactlens technologie. Een probleem bij de eerste generatie materialen vormde de geometrische instabiliteit en aanslag van stoffen op het oppervlak waardoor er een zekere terughoudendheid is ontstaan in de toepassing van deze lenstypen. Een soortgelijke ontwikkeling deed zich voor bij de introductie van asferische lensontwerpen. De technologische vooruitgang in zowel de productie van het contactlensmateriaal, alsook het vervaardigen van de uiteindelijke lens met behulp van computer gestuurde (CNC) technieken en nieuw ontwikkelde aanmeet- en onderhoudssystemen, was aanleiding om de klinische resultaten van moderne HGP contactlenzen te onderzoeken.

In een onderzoek bij 68 patiënten vergeleken wij gedurende 1 jaar het succes van sferische en asferische lensontwerpen met een laag en hoog zuurstofdoorlatend materiaal (3.2). In $77 \%$ van de gevallen werden de lenzen met succes de gehele dag gedragen, in $11 \%$ was er sprake van een beperkte draagtijd en in $12 \%$ werd het dragen van de lenzen gestopt. Een geringere draagtijd of stoppen met dragen was gerelateerd aan draagcomfort, uitdrogen van het lensoppervlak en cornea epitheel afwijkingen (3-9 uur kleuringen). Tussen de verschillende lensontwerpen en de gebruikte materialen werden zowel voor de klinische criteria als voor de stabiliteit van de lenzen geen significante verschillen gevonden. Dit betekent dat er geen technische belemmering meer is om hoog zuurstofdoorlatende materialen in een asferisch ontwerp toe te passen.

De gunstige resultaten uit het voorgaande onderzoek waren aanleiding om de aanmeet- en onderhoudsmethoden van asferische HGP contactlenzen nader te onderzoeken (3.3). In dit onderzoek werd bij 66 patiënten een hoog zuurstofdoorlatende contactlens met een asferisch 
ontwerp aangemeten. Random geselecteerd gebruikten we twee aanmeetmethoden en kregen de patiënten twee verschillende onderhoudssystemen. Na 1 jaar follow-up bleek 85\% van de patiënten de lenzen de gehele dag succesvol te dragen, $7.5 \%$ droeg de lenzen beperkt en $7.5 \%$ was gestopt met dragen. Beperkt dragen of stoppen was gerelateerd aan draagcomfort, het uitdrogen van het lensoppervlak en 3-9 uur kleuringen. Een door ons ontwikkeld selectieschema bleek de uiteindelijk succesvol gebruikte lensdiameter significant beter $(90 \%)$ te voorspellen dan de methode waarbij de maat van de verticaal zichtbare iris onder supraversie wordt gebruikt (72\%). Een opvallend resultaat in dit onderzoek was dat de topografische methode om de basiscurve van de lens te voorspellen het slechter (75\%) deed dan de methode waarbij men slechts de centrale keratometerwaarden en een vuistregel gebruikt $(83 \%)$. Hoewel het verschil niet significant was zou men toch verwachten dat de topografische methode het juist beter zou doen. Bij nadere analyse van de corneatopografie bleek dat bij onjuiste voorspellingen het verschil in afvlakking (E-waarde) van de cornea tussen haar kwadranten significant groter was dan bij juiste voorspellingen. Het toepassen van een rekenmethode gebaseerd op gewogen gemiddelden van de cornea-afvlakkingen in vier kwadranten zou het succes in het voorspellen van de basiscurve via de methode van topografie theoretisch kunnen verhogen tot $92 \%$. Hoewel de predictieve waarde van dit nieuw ontwikkelde systeem hoog lijkt, is deze onvoldoende om te worden gebruikt als een systeem voor direct bestellen zonder een controle door een competente contactlensspecialist. $\mathrm{Na} 1$ jaar gebruik bleek dat zowel het onderhoudssysteem met een mechanische reinigingsvloeistof (79.6\%) als de alles-in-één vloeistof (79.4\%) de lenzen in goede conditie konden houden met betrekking tot zowel eiwitaanslag als stabiliteit. Het gebruik van een alles-in-één vloeistof lijkt de beste optie om een goed dagelijks onderhoud te waarborgen.

Wij concluderen dat de vormstabiele hoog zuurstofdoorlatende asferische contactlens succesvol kan worden toegepast mits goed gefabriceerd, efficiënt aangemeten en goed onderhouden. De tussenkomst van een contactlensspecialist blijft bij de lensselectie, het aanmeten en vervolgen een belangrijke factor voor succes.

\section{Hoofdstuk 4}

Moderne CNC fabricagetechnieken maken het mogelijk om de toepassingen van hydrofiele zachte contactlenzen uit te breiden met de optische correctie van astigmatisme, presbyopie, sferische aberratie bij hoge ametropie en extreme optische aberraties zoals bij keratoconus.

Het onderzoek naar zachte torische custom-made lenzen liet zien dat een relatief eenvoudig systeem van bestellen vanuit oogparameters succesvol kan worden gebruikt (4.2). Dit onderzoek omvatte 193 ogen met astigmatisme tussen 1 en 7.5 dioptrie. Zachte torische contactlenzen werden vervaardigd op basis van refractiewaarden, cornea radii, en horizontaal zichtbare iris diameter. Het succespercentage van deze lenzen was $69 \%$ voor nieuwe lensdragers en $54 \%$ voor patiënten die al eerder torische zachte lenzen (zonder succes) hadden geprobeerd. In de gevallen waarbij succes niet direct aanwezig was kon bij nieuwe patiënten met het bestellen van een tweede of derde lens het succespercentage worden verhoogd tot $92 \%$. Bij de groep patiënten die al eerder torische lenzen hadden geprobeerd was dit percentage 
significant lager (74\%). Met deze resultaten lijkt het erop dat het problematisch aanmeten met behulp van paslenzen kan worden vermeden. Echter, vanuit onze resultaten is het ook duidelijk dat een dergelijk systeem nog niet voldoende sterk is om te gebruiken als een rechtstreeks bestelsysteem zonder controle van een contactlensspecialist.

In onze studie naar de toepassing van contactlenzen voor de correctie van presbyopie konden we met intensief literatuuronderzoek bijdragen aan de beschrijving van de complexe situatie rond dit meer en meer actueel wordende onderwerp (4.3). De selectie uit 5 verschillende prototypen varifocale contactlenzen leverde, in een klinisch onderzoek met 90 patiënten, een uiteindelijk succespercentage op van $67.8 \%$. Het succes bleek voor het zien in de verte uitsluitend samen te hangen met het optische profiel van de lenzen en bij het nabij-zien met een combinatie tussen benodigde additie en pupilgrootte. Belangrijk is te concluderen dat met de speciale ontwerpen die wij gebruikten het succes in de correctie van de presbyopie in het algemeen kon worden verbeterd en dat het visueel functioneren geoptimaliseerd kan worden indien het profiel van de lens individueel zou worden ontworpen. De resultaten van ons onderzoek met speciaal ontworpen asferische lenzen toonden bij 122 ogen met hoge ametropie aan dat een systematische correctie van sferische aberratie gemiddeld genomen geen zin heeft (4.4). Echter, in ongeveer $30 \%$ van de gevallen was er sprake van een verbetering in de contrastgevoeligheidsfunctie. Dit betekent dat indien men in staat is de sferische aberratie van het individuele oog te meten dit met de tegenwoordige technologie ook te corrigeren is.

De theoretische mogelijkheden voor het corrigeren van extreme optische aberraties hebben wij onderzocht aan de hand van de cornea-topografie bij 9 ogen met keratoconus (4.5). Bij perfect gecentreerde lenzen kan de contrastgevoeligheidsfunctie, afhankelijk van de mate van optische aberratie, met een factor tussen 6.5 tot 200 worden verbeterd. Beweging en rotatie van de lens op het oog kan deze winst echter weer teniet doen. Bij rotatie tot 10 graden blijft de winst een factor 3 of hoger. Bij beweging van de lens in $\mathrm{x}$ en $\mathrm{y}$ richting ligt de kritische grens voor het behalen van visuele winst bij $0.5 \mathrm{~mm}$.

In dit hoofdstuk hebben we aangetoond dat de introductie van nieuw ontwikkelde zachte hydrofiele lenzen de optische correctiemogelijkheden aanzienlijk heeft uitgebreid. De goede centrering en geringe beweging van dit type contactlens biedt unieke kansen om hogereorde optische aberraties te corrigeren. Het blijkt echter dat visuele winst slechts te behalen is door de optische eigenschappen van het oog nauwkeurig te meten en de correctielenzen individueel te ontwerpen en te fabriceren. Ook hier is de tussenkomst van een contactlensspecialist essentieel voor succes.

\section{Hoofdstuk 5}

Zoals eerder beschreven vereisen de nieuwe technische mogelijkheden om het oog met behulp van contactlenzen te corrigeren ook gedetailleerde metingen. In dit hoofdstuk rapporteerden wij over de ontwikkeling van nieuwe instrumenten en methoden om het oog te meten en het voorste oogoppervlak te evalueren.

De evaluatie van de Maastricht Shape Topographer (MST) liet zien dat dit instrument in staat is om de topografie van het voorste oogsegment rechtstreeks te meten (5.2.1). Deze op 
Fourier-profilometrie gebaseerde methode is in staat om met een hoge nauwkeurigheid 20 x $16 \mathrm{~mm}$ van het voorste oogvlak als hoogtekaart of als meridionale doorsnede te presenteren.

De studie met de MST naar een vergelijking tussen het cornea-scleraprofiel bij normale en keratoconus ogen (5.2.2) toonde aan dat bij keratoconus de asymmetrie in de stand van de limbus, zoals gevonden voor normale ogen, niet aanwezig is. De meridionale doorsneden van het totale oogvlak kunnen een goed uitgangspunt vormen voor het ontwerpen van (semi)-sclerale contactlenzen.

Het onderzoek met een door ons ontwikkelde pupillometer (5.3) liet een aantal belangrijke karakteristieke kenmerken zien van de pupil bij visuele taken in de verte en bij het lezen onder verschillende lichtomstandigheden. Deze gegevens kunnen in belangrijke mate bijdragen tot een beter ontwerp van met name varifocale contactlenzen.

Het laatst beschreven onderzoek naar de fenomenen van fluorescentie (5.4) laat zien dat een goed gekozen combinatie van excitatiegolflengte in combinatie met type, concentratie en substraat van de fluoresceïne, het mogelijk maakt om oculaire structuren naar klinische wens zichtbaar te maken en de betrouwbaarheid van hoogtetopografie van het voorste oogvlak te verhogen.

Samenvattend toont dit proefschrift aan dat er met contactlenzen tegenwoordig een grote variatie aan excellente opties aanwezig is om het oog te corrigeren. De implementatie van nieuwe technologische ontwikkelingen levert positieve resultaten op in het aanmeten van contactlenzen, in de fysiologische reacties en in efficiëntie van het onderhoud. Verbeterde technieken om het oog te meten, computer-aided lensontwerp en computer numeriek bestuurde fabricagemethoden maken het mogelijk om bijna alle optische afwijkingen van het oog met inbegrip van hogere-orde aberraties te corrigeren.

Onze studies tonen tevens aan dat de betrokkenheid van de contactlens specialist een voorwaarde is voor een volledige ontplooiing van deze nieuwe ontwikkelingen. Het werk van de specialist zal in de toekomst sterk verschuiven van passen en modificeren naar meten, ontwerpen en vervolgen. Het gebruik van de in deze studie ontwikkelde objectieve meet-, ontwerp- en evaluatiemethoden kan daarbij een waardevol hulpmiddel zijn. 


\section{Dankwoord}

Een proefschrift is het formele bewijs dat iemand in staat is om zelfstandig de wetenschap te bedrijven en tevens de ideeën die daaruit zijn ontstaan te verdedigen. Daarmee wil ik op voorhand de Corona bedanken voor de lastige vragen die zij mij zullen stellen.

Het promoveren heb ik als een bijzonder leerproces ervaren waarbij denken, voelen en willen voortdurend op de proef werden gesteld. Zoiets kan slechts geschieden door op allerlei gebied met andere mensen samen te werken. Mijn dank gaat daarom uit naar iedereen die op welke wijze dan ook heeft bijgedragen aan het tot stand komen van dit proefschrift.

Een aantal mensen wil ik in het bijzonder noemen.

Mijn ouders. Ik dank jullie hartelijk voor de fantastische kindertijd en de geboden mogelijkheden om mijn eigen plaats op deze aarde te vinden.

Mijn vakopleiders dank ik voor de enthousiaste overdracht van de theorie rond visuele waarneming en de praktijk van de correctie daarop. Door jullie ben ik van dit vak gaan houden.

Wim Aalbers en Jaap van Blitterswijk. We zijn er in geslaagd om de zachte contactlens met succes in Nederland te introduceren. Teamwork, maar vooral creativiteit heb ik van jullie geleerd. We woonden soms "bijna samen" en dat was de basis voor een vriendschap voor het leven.

Dr. Jan Kok. In de tijd dat ik werkzaam was in het AMC te Amsterdam was het Dr. Jan Kok, toen zelf promoverend op het onderwerp contactlenzen, die mij aanzette om het onderzoek verder te verdiepen. We hebben veel samengewerkt. Getuigenis daarvan zijn een groot aantal artikelen, waarvan enkele in dit proefschrift, maar bovenal een enorm aantal tevreden patiënten die door zijn inventiviteit een oplossing vonden voor moeilijke oogproblemen. Jan, het doet mij nog dagelijks verdriet dat ik je postuum moet bedanken. Je blijft voor altijd in mijn hart.

Het AMC-research team. Onderzoek naar contactlenzen werd in het AMC in teamverband gedaan. Dit team vormde voor mij een enorme stimulans en ik ben hierdoor definitief gevallen voor het vak van onderzoeker. Cor van Mil bedank ik voor de prettige samenwerking in de kliniek. Ik vond het geweldig dat ik jou jaren daarvoor iets van het vak mocht leren en dat in de AMC-tijd het omgekeerde het geval was. Dr. Cees Brinkman dank ik in het bijzonder voor zijn gave om de relatie tussen klinische observaties en de statistische uitwerking daarvan duidelijk te maken. Cees, ik dank je dat jij mij hebt geleerd om bij het altijd moeilijke klinische onderzoek vooraf de statistische power in het oog te houden. Dr. Liesbeth Wenniger-Prick dank ik voor de bijzonder prettige manier waarop we hebben samengewerkt aan het onderzoek met zeer jonge kinderen. Liesbeth, als orthopedagoog wil ik je complimenteren met de kwaliteit die jij hebt om met kinderen en hun ouders om te gaan. Prof. Dr. Aize Kijlstra, samen met Dr. Jan Kok de motor achter het AMC-researchteam, bedank ik voor de manier waarop hij de wetenschappelijke inspiratie in de club tot een hoogtepunt bracht. Aize, ik stel het op prijs dat onze wegen in Maastricht weer samen zijn gekomen.

Dr. Nicolas Chateau. Many investigations in this thesis are conducted in co-operation with Dr. Nicolas Chateau from Paris (researcher at Ocular Sciences, former Essilor Optique de Contact). Nicolas, during the many years we work together, I very much appreciated your extensive knowledge of optics and the way you were able to transform clinical questions into fundamental research. I thank you very much for your enthusiastic way to stimulate rese- 
arch and to put this into publications. Finally, we succeeded to initialise the CLAAS project that could become one of the major achievements in the development of contact lenses in the next years. Most important to me, is the fact that we have become friends.

Mijn overige partners bij het onderzoek, Tino Sax, Eric Brookman, Henk Molenaar, Florence Bouchard, Sandrine Guidollet, Gildas Marin, Pablo Artal, Norberto Lopez-Gil, Antonio Benito, Eef van der Worp, Bertho Stultiens, Guido Mols en Jeroen van Rooij dank $\mathrm{ik}$ voor de fantastische samenwerking. Jullie inzet heeft er toe bijgedragen dat de onderzoeken niet alleen werden volbracht maar ook konden worden gepubliceerd. In dit verband wil ik met nadruk ook bedanken, alle medewerkers van de oogheelkundige poli's en de contactlensinstituten waar ik mijn onderzoeken mocht doen. Zonder jullie inzet om heel consequent patiënten te screenen, te ontvangen en te instrueren zou het nooit gelukt zijn om mijn onderzoeken te volbrengen.

De basis voor de onderzoeken in dit proefschrift vormde de technologische vooruitgang in de contactlenswetenschap. Zonder de beschikbaarheid van concrete producten of technieken zou er weinig te onderzoeken zijn geweest. Ik dank daarom de volgende bedrijven voor hun welwillende technische bijdrage in de vorm van producten, technieken of informatie: Bausch \& Lomb, Igel International, Oculenti, Microlens, Polymer Technology, Procornea, NKL, ICN Pharmaceuticals, Essilor, Ocular Sciences, Sumipro en Euro Precision Technology.

Ing. Astrid Hacking, dank ik voor de constructieve samenwerking binnen het onderzoeksinstituut, Eye Research Institute Maastricht (ERIM).

Dr. Frans Jongsma. De reden dat ik als onderzoeker bij de Universiteit Maastricht ging werken was de uitvinding van de Maastricht Shape Topographer (MST) door Frans Jongsma. Frans, vanaf het eerste moment dat je mij de achtergronden van de MST hebt uitgelegd heb ik mij enorm bij je thuis gevoeld. Ik snapte direct waar je, als kernpunten van het door jou als opticus ontwikkelde systeem, over sprak. Het was daarom ook een genoegen om jouw promotie over dit onderwerp te ondersteunen. In de jaren dat we nu samenwerken bedank ik je vooral voor je veelzijdigheid om een probleem fundamenteel-analytisch, exploratief of pragmatisch te benaderen. Het doet mij zeer veel genoegen om je nu naast vriend en collega ook nog als co-promotor te mogen bedanken.

Dr. Rudy Nuijts, mijn andere co-promotor, heeft een essentiële rol gespeeld bij het tot stand komen van de uiteindelijke vorm van dit proefschrift. Rudy, ik bedank je voor de stimulans om ideeën steeds verder uit te werken en om je vriendschappelijke maar toch altijd zeer kritische houding bij het analyseren van de resultaten en het op schrift stellen daarvan.

Prof. Dr. Fred Hendrikse, mijn promotor, dank ik voor het creëren van een structuur waarin ik een bijdrage kon leveren aan het verder uitbouwen van oogonderzoek aan de Universiteit Maastricht. Fred, ik waardeer het bijzonder dat je als retina specialist mijn optometrisch / technisch onderzoek hebt gestimuleerd en begeleid.

Lieve Petra, Merijn, Coby, Nayomi, Francisco, Roberto, Victor, Françoise, Kees, Frieda en Yves. Dit boek is nu het resultaat van mijn bezigheden die voor jullie soms vaag waren maar vaak laat in de avond, vroeg in de ochtend, of in de weekends werden uitgevoerd. Toch hebben jullie een belangrijke bijdrage geleverd omdat wetenschap alleen een arm leven zou zijn en dit werk dan waarschijnlijk niet eens was gestart.

Loni, jij bent in vele opzichten mijn wederhelft. Dank drukt niet uit wat ik voel als jouw bijdrage. Het is gewoon een wonder en ik verheug me op onze verdere reis. 


\section{Curriculum vitae}

John de Brabander is geboren op 22 september 1946 te Delft en behaalde zijn diploma contactlensspecialist in 1969 aan de Christiaan Huygensschool te Rotterdam. Hij startte zijn carrière bij Biessels, Den Haag en specialiseerde zich in refractie, low vision en contactlenzen.

Vanaf 1971 tot 1975 werkte John voor Bausch \& Lomb, waardoor hij nauw betrokken was bij onderzoek, ontwikkeling en introductie van de eerste generatie zachte contactlenzen. Als professional relations manager Europa en het Midden-Oosten was hij een van de initiators en organisatoren van de "European Symposia on Contact Lenses".

Van 1975 tot 1985 was hij docent en cursuscoördinator aan de opleiding voor optometristen en contactlensspecialisten. Hij was mede oprichter van de European Association of Schools in Optometry, de eerste secretaris van de International Association of Contact Lens Educators, en vice-voorzitter van de Optometristen Vereniging.

Vanuit zijn belangstelling voor het onderwerp leerstoornissen studeerde John aan de Rijks Universiteit, Groningen en behaalde aldaar zijn doctoraalexamen orthopedagogiek.

Hij werkte van 1989 tot 1995 voor ICN/Oculenti als adviseur voor onderzoek en productontwikkeling en vanaf 1993 tevens als onderzoeker in het Academisch Medisch Centrum bij de Universiteit van Amsterdam.

Vanaf 1996 is John werkzaam als onderzoeker aan de Universiteit Maastricht, afdeling oogheelkunde van het AZM en het Eye Research Institute Maastricht (ERIM). Zijn onderzoeksactiviteiten omvatten het ontwikkelen van meet- en beeldtechnieken ten behoeve van diagnostiek en behandeling van het oog. 


\section{Publications described in this thesis}

\section{Chapter 2}

\section{Section 2.2}

Brabander de J, Kok JHC, Nuijts RMMA, and Wenniger-Prick LJJM. A Practical Approach to and Long Term Results of Fitting Silicone Contact Lenses in Aphakic Children After Congenital Cataract.

CLAO J 2002;28;1:31-5.

Section 2.3

Brabander de J, Mil van CJ, Brinkman CJJ and Nuijts RMMA. The use of Standard thin high Water Content Artificial Iris and Black Pupil Contact Lenses.

Digital Journal of Ophthalmology 2001;7:No 1. www.djo.harvard.edu.

\section{Chapter 3}

Section 3.2

Brabander de J, Brinkman CJJ, Kok JHC and Mil van CJ. Low or High Dk? Spherical or Aspherical Rigid Gas Permeable Lenses? The Gap between Theory and Practice.

J BCLA, 1992;15;4:159-62.

\section{Section 3.3}

Brabander de J, Kok JHC and Nuijts RMMA. Evaluation of a New System of Lens Parameter Selection and Comparison of Traditional vs One-Step lens Care Systems for Aspheric High-Dk RGP Contact Lenses.

CLAO J 2000;26;4:193-9.

\section{Chapter 4}

Section 4.2

Brabander de J, Brinkman CJJ, Nuijts RMMA, Mil van CJ, Sax T and Brookman E. Clinical evaluation of a Custom made Toric Soft Lens System.

Contact Lens \& Anterior Eye, 2000;23;1:22-8.

\section{Section 4.3}

Brabander de J, Chateau N, Molenaar H and Bouchard F. Presbyopia and Contact Lenses: A Literature Review and a Clinical Study on Discriminant Factors in Varifocal Soft Contact Lens Fitting.

Contactologia 2000;22;99-108.

\section{Section 4.4}

Brabander de J, Chateau N, Bouchard F and Guidollet S. Contrast Sensitivity with Soft Contact Lenses Compensated for Spherical Aberration in High Ametropia.

Optom Vis Sci 1998;75;1:37-43.

\section{Section 4.5}

Brabander de J, Chateau N, Artal P, Marin G, Lopez-Gil N, Worp van der E and Benito A. Simulated Optical Performance of Custom Wave-Front Soft Contact Lenses for Keratoconus.

Submitted. 


\section{Chapter 5}

\section{Section 5.2.1}

Jongsma FHM, Brabander de J, Hendrikse F and Stultiens BAT. Development of a Wide Field Height Eye Topographer: Validation on Models of the Anterior Eye Surface.

Optom Vis Sci 1998;75;1:69-77.

\section{Section 5.2.2}

Brabander de J, Nuijts RMMA, Jongsma FHM, Hendrikse F. Height Measurements of the Corneal-Scleral profile using the Maastricht Shape Topographer.

Submitted.

\section{Section 5.3}

Chateau N, Brabander de J, Bouchard F and Molenaar H. Infrared Pupillometry in Presbyopes Fitted with Soft Contact Lenses.

Optom Vis Sci 1996;73;12:733-41.

\section{Section $\mathbf{5 . 4}$}

Brabander de J, Jongsma FHM, van Rooij JIJH and Hendrikse F. Testing Fluorescein Agents as a Diagnostic Tool and a Coating for Anterior Eye Topography: A Feasibility Study.

Submitted.

\section{Other publications}

Brabander de J, Koppenberg B, Lent van AJG, Mosch H, Ringenoldus WFMT, Tierolf PJ. De plaats van automatische refractie instrumenten in de optometriepraktijk. Optometrie 1980;1;1:8-19.

Brabander de J. Is verzorging van contactlenzen eenvoudig voor de meeste of moeilijk voor allemaal?

Optical Vision;1990;3;1:14-16.

Brabander de J. Contact lens materials.

Optical Vision;1990;3;3:23-25.

Brabander de J, Jongsma FHM, Heijde van der GL. Contact Lenses, Small in Optics. Kontaktlenzen, kleintjes in de optica.

Klinische Fysica;1994;3:314-19.

Brabander de J, Chateau N, Molenaar H, Bouchard F. Discriminant factors in multifocal soft contact lens fitting. Preliminary results.

Contactologia;1996;18;3:138-40.

Jongsma FHM, Brabander de J, Hendrikse F. Meten aan het hoornvlies. NTvG 1997;63/2:39-43. 
Brabander de J. Topografie van het oog op basis van hoogte-metingen. Eye Line 1998;4:27.

Jongsma FHM, Brabander de J, Hendrikse F. Ontwikkeling van de Euclid oogtopograaf. Visus; 1998;4:12-7.

Jongsma FHM, Brabander de J, Hendrikse F. Review and Classification of Corneal Topographers.

Laser Med Sci 1999;14:2-19.

Bruining MJ, Blaauwgeers HGT, Kuijer R, Jongsma FHM, Brabander de J, Nuijts RMMA, Koole L. Tailoring of new Polymeric Biomaterials for the Repair of Medium-Sized Corneal Perforations.

Biomacromolecules 2000;1;3:418-23.

Brabander de J, Brinkman CJJ, Nuijts RMMA, Mil van C, Sax T, Brookman E. Klinische evaluatie van een maatsysteem voor torische zachte contactlenzen.

Visus 2000;3:75-80. 


\section{List of abbreviations and major terms}

Abbreviation

AI

AIr

AR

$\mathrm{AO}$

AROC

AF

ASPH

$\mathrm{BC}$

BCR

BCOR

BOZR

BOZD

BT

BUT

Button

$\mathrm{CAB}$

CAVK

\section{Short Description or Explanation}

Astigmatismus Inversus. Against the rule astigmatism. Corneal astigmatism such that the principle meridian with the highest (plus) dioptric power lies between 0 and 22.5, or, 180 and 157.5 degrees TABO.

Astigmatismus Irregularis, irregular astigmatism. Astigmatism of the eye in which the optical properties cannot completely be captured by two principle meridians lying 90 degrees apart.

Astigmatismus Rectus. With the rule astigmatism. Corneal astigmatism such that the principle meridian with the highest (plus) dioptric power lies between 67.5 and 112.5 degrees TABO.

Astigmatismus Obliquus. Oblique astigmatism. Corneal astigmatism such that the principle meridian with the highest (plus) dioptric power lies between 22.5 and 67.5 , or, 112.5 and 157.5 degrees TABO.

Axial Radius of Curvature. Radius of curvature of an area anywhere on a surface which origin is at the principle axis.

Alignment Fit. Contact lens posterior surface that follows the corneal anterior surface.

Aspheric. Surface that deviates from a sphere.

Bi-Curve. Contact lens with anterior geometry having two rotational symmetric curves.

Base Curve Radius. Obsolete term for BOZR.

Back Central Optic Radius. Obsolete term for BOZR.

Back Optic Zone Radius. Radius of curvature of the back optical zone of a contact lens.

Back Optic Zone Diameter. Diameter of the posterior central zone in single vision contact lenses that has a prescribed optical effect.

Bi-Toric. Contact lens of which both posterior and anterior surfaces have two different spherical (principle) meridians at 90 degrees from each other.

Break Up Time. See TBUT.

Blank. Cylindrical piece of polymerised material from which a contact lens is manufactured by lathe cutting.

Cellulose Acetate Butyrate. One of the first (low) oxygen permeable thermoplastic CL materials; derivative of cellulose by substituting hydroxyl groups by acetyl and butyril radicals.

Computer Aided Video Keratometry. Group of devices that by the aid of a computer uses algorithms to analyse Placido type of images in order to estimate corneal shape. 
$\mathrm{CM}$

CL

CLAAS

CLMA

$\mathrm{CNC}$

CW

CC

CCD

CSF

CSP

$\mathrm{D}$

DDisp.

Dep.

$\mathrm{Dk}$

$\mathrm{Dk} / \mathrm{L}$

DW

EOP

EC

EFCLIN

EW
Cast Moulding. Manufacturing technique for plastics in which liquid ingredients are injected in a preformed mould.

Contact Lens. Relatively small and thin lens that can be worn on the eye.

Contact Lenses with Aspheric and Asymmetric Surfaces. Research project funded by the EC with the aim of producing contact lenses with aspheric and asymmetric surfaces incorporating lens geometry's optimised to the corneal shape and the optical properties of the individual eye.

Contact Lens Manufacturing Association. Organisation of contact lens manufacturers.

Computer Numeric Controlled. Manufacturing technique guided by numeric machine codes.

Continues Wear. Wear of contact lenses daily and over night for an indefinite period.

Continues-Curve. Posterior surface of a contact lens described by a mathematical expression in which no transitions occur.

Charge Coupled Device. Light sensitive chip containing many sensitive elements (pixels) used in video cameras.

Contrast Sensitivity Function. Ability of the visual system to discriminate patterns defined by spatial frequency and contrast.

Corneal-Scleral Profile.

Dioptre. Unit of refractive power of an optical surface.

Daily Disposables. Contact lenses that are used for one day and than disposed.

Deposits. Substances adhered to the contact lens surface, usually in denatured form.

Oxygen Permeability. Value that expresses the ability of a material to permit the passage of oxygen in which $\mathrm{D}$ is the diffusion and $\mathrm{k}$ the solubility coefficient.

Oxygen Transmissibility. Value that expresses the amount of oxygen that can be transmitted through a material with a specified thickness (L).

Daily Wear. Wearing of contact lenses limited to waking hours.

Equivalent Oxygen Pressure. Value that expresses the state of oxygen tension of the cornea by measuring the oxygen uptake directly after removing a contact lens.

European Commission. Organisation that develops and executes rules that are stated of importance to the European community.

European Federation of Contact Lens Industry. Also Euromcontact. Organisation of European contact lens manufacturers.

Extended Wear. Wearing of contact lenses beyond waking hours but limited in time. 
E-value

FASAM

FDA

Flat fit

Flexi lens

FRL

FT

FW

GP

GPC

HGP

HEMA

IACLE

IT

IROC

ISO

K-reading

Maréchal criterion
Eccentricity. Value that expresses the deviation from the ground circle in conic sections.

FluoroAlkyl/SiloxanylAlkyl Methacrylate. Fluoro-silicon-acrylates, a group of copolymers in which combinations of monomers including fluoromonomers, copolymers and cross linking agents are used to enhance oxygen permeability and material properties.

Food and Drug Administration. Organisation that develops and executes rules concerning safety for use of products in the United States of America.

Also loose fit, flatter than K. A contact lens which posterior surface shows in vivo a tear layer with increasing thickness toward the periphery.

Also mini, Boyd lens. Small, thin PMMA contact lens.

Frequent Replacement Lenses. Scheme of prospectively planned replacement of contact lenses, e.g. 6-months, 3-months, monthly, weekly.

Front-Toric. Contact lens with an anterior surface having two principle meridians of different radius of curvature lying 90 degrees from each other.

Flexible Wear. Wearing of contact lenses adopted to the need or desire of the individual patient.

Gas Permeability. Value that expresses the ability of a material to permit the passage of gasses. See also oxygen permeability.

Giant Papillary Conjunctivitis. Immunological responds of the palpebral conjunctiva to foreign materials.

High Gas Permeable. Group of contact lenses made out of a material with high oxygen permeability (Dk). The definition of "high" is arbitrary and has shifted with time (see ISO).

HydroxyEthylMethAcrylate. Monomer with hydrophilic properties. International Association of Contact Lens Educators.

Inside-Toric. Contact lens with a posterior surface having two principle meridians of different radius of curvature lying 90 degrees from each other.

Instantaneous Radius of Curvature. Radius of curvature of an area anywhere on a surface without the necessity that the origin is at the principle axis.

International Organization for Standardization.

Keratometric value. Average radius of curvature of a limited central area of the cornea as can be estimated by comparison of the size of a known object to its image as reflected by the cornea.

Critical pupil size for which the root-mean-square (RMS) of a present wavefront deviates from that of a perfect sphero-cylinder by onefourteenth of the wavelength of the light source used. 
$\mathrm{MC}$

MST

NAC

NVP

$\mathrm{OH}$

PW

Placido

Pix.

Purkinje

PMMA

RA

RGP

Sag.

SAM

SCL

SE

Steep fit
Multi-Curve. Contact lens posterior surface that contains more than one curve all having their centre of origin on the same central axis. Maastricht Shape Topographer. Device to measure the anterior eye surface.

Nederlandse Associatie van Contactlensleveranciers. Dutch organisation of CL suppliers.

$\mathrm{N}$-Vinyl-Pyrrolidone. Hydrophilic monomer used to increase hydration and oxygen permeability in CL.

Hydroxyl group. Chemical group of oxygen and hydrogen that is able to bound with water.

Permanent Wear. Wearing of contact lenses daily and overnight for an indefinite period.

Placido disk. After Placido, inventor of a flat disk with concentric black and white circles and a central hole to qualitatively estimate optical properties of the corneal surface (basis for CAVK).

Pixel. Principle elements of a picture.

Purkinje image(s). Images from objects in space formed by the surfaces of the cornea and crystalline lens in the eye (Purkinje images I to IV).

PolyMethylMethAcrylate. Homopolymer of methylmethacrylate (MMA).

Refractive index. Unit that expresses the speed of light that can be reached in a medium relative to the speed of light in a vacuum.

Residual, Rest Astigmatism. Astigmatism that remains after an attempt to completely correct the eye.

Rigid Gas Permeable. Contact lens made of a rigid material capable to retain its form without support and of providing substantial corneal oxygenation by flow through the material.

Sagittal depth or height. Perpendicularly measured distance between the line that cuts a segment from a rotational symmetric shape and its highest point.

SiloxanylAlkyl Methacrylate. Silicon-acrylates. A group of copolymers in which combinations of monomers, copolymers and cross linking agents are used to enhance oxygen permeability and material properties.

Soft Contact Lens. Contact lens that requires support to maintain its form.

Sphere Equivalent. Average dioptric power of a toric surface, lens or corrective prescription.

Also tight fit, steeper than K. A contact lens which posterior surface shows in vivo a tear layer with decreasing thickness toward the periphery. 
Strehl ratio Ratio of the light intensity at the peak of the diffraction pattern of an aberrated image to that of the peak of an aberration free image.

Stiles-Crawford effect The different effects of energy transformation related to the angle of incidence of the optical rays approaching the retina.

TABO Technisches Ausschuss Brillen Optik. Normative system to define the direction in which cylindrical or prismatic spectacle corrections should be placed.

TBUT Tear film Break Up Time. Time between a blink and the first disruption of the tear film.

TC Tetra-Curve. Contact lens design in which the posterior surface is build from four curves.

TD Total Diameter. Overall diameter of a contact lens.

VA Visual Acuity. Measure of the ability of the visual system to discriminate defined high contrast objects in space, expressed directly as the (logarithmic) angle that the smallest recognisable object subtends on the retina (minimal angle of resolution, $\log$ MAR) or relative to a standard angle of 1' of arc (Snellen VA). 
